\title{
Modulation of Semiconductor Photoconversion with Surface Modification and Plasmon
}

\author{
Joeseph Martin Bright \\ West Virginia University, jbright3@mail.wvu.edu
}

Follow this and additional works at: https://researchrepository.wvu.edu/etd

Part of the Semiconductor and Optical Materials Commons

\section{Recommended Citation}

Bright, Joeseph Martin, "Modulation of Semiconductor Photoconversion with Surface Modification and Plasmon" (2020). Graduate Theses, Dissertations, and Problem Reports. 7798.

https://researchrepository.wvu.edu/etd/7798

This Dissertation is protected by copyright and/or related rights. It has been brought to you by the The Research Repository @ WVU with permission from the rights-holder(s). You are free to use this Dissertation in any way that is permitted by the copyright and related rights legislation that applies to your use. For other uses you must obtain permission from the rights-holder(s) directly, unless additional rights are indicated by a Creative Commons license in the record and/ or on the work itself. This Dissertation has been accepted for inclusion in WVU Graduate Theses, Dissertations, and Problem Reports collection by an authorized administrator of The Research Repository @ WVU.

For more information, please contact researchrepository@mail.wvu.edu. 
Modulation of Semiconductor Photoconversion with Surface Modification and Plasmon

Joeseph Martin Bright

Follow this and additional works at: https://researchrepository.wvu.edu/etd

Part of the Semiconductor and Optical Materials Commons 


\title{
Modulation of Semiconductor Photoconversion with Surface Modification and Plasmon
}

\author{
Joeseph Bright
}

\begin{abstract}
Dissertation submitted
to the Benjamin M. Statler College of Engineering and Mineral Resources

at West Virginia University
\end{abstract}

in partial fulfillment of the requirements for the degree of

Doctor of Philosophy

in

Materials Science and Engineering

Nianqiang Wu, Ph.D. (Co-Chair)

Terence Musho, Ph.D. (Co-Chair)

Ever J. Barbero, Ph.D.

Lawrence Hornak, Ph.D.

Dongling Ma, Ph.D.

Department of Mechanical and Aerospace Engineering

West Virginia University

Morgantown, West Virginia

2020

Keywords: Plasmonics, Solar Energy, Photoelectrochemistry, Sensors, Heavy Metals, Immunosensor

Copyright 2020 - Joeseph Bright 


\section{Abstract}

\section{Modulation of Semiconductor Photoconversion with Surface Modification and Plasmon}

\section{Joeseph Bright}

Semiconductor devices are the basis of modern technology. Semiconductor-based photoconversion devices that convert light into electrical signals have shown potential for light energy harvesting and conversion, environmental remediation, and sensors for detection of light, chemicals, and biological substances. Despite this potential for use in many applications, semiconductor photoconversion devices need further improvement in the photoconversion performance. This photoconversion improvement may be manifested as increased photoconversion efficiencies for light harvesting devices for power generation such as photovoltaics and photoelectrochemical (PEC) cells or improved photoconversion modulation to increase the sensitivity of semiconductor photoconversion-based sensors. In addition, alternative semiconductor materials to semiconductors that utilize toxic heavy metals such as cadmium and lead must be found for use in certain semiconductor photoconversion devices.

In this dissertation, three separate projects related to improving the performance of semiconductor photoconversion devices are presented. In the first project presented, a rutile titanium dioxide $\left(\mathrm{TiO}_{2}\right)$ nanorod array photoanrode is coated with an ultra-thin porphyrin-based metal-organic framework (MOF) layer to improve the overall photoconversion of the photoelectrode for solar water splitting. The porphyrin-based $\mathrm{MOF}$ coated $\mathrm{TiO}_{2}$ nanorod array showed a $2.7 \mathrm{x}$ increase in photocurrent versus bare $\mathrm{TiO}_{2}$ nanorod arrays. The porphyrin-based MOF layer suppressed surface states on the rutile $\mathrm{TiO}_{2}$ nanorod array and increased charge separation and extraction from the rutile $\mathrm{TiO}_{2}$ due to the built-in electric field formed by a depleted p-n junction between the porphyrin-based MOF layer and the rutile $\mathrm{TiO}_{2}$ nanorods.

In the second project presented, different plasmonic (hot electron injection and plasmoninduced resonant energy transfer (PIRET)) and non-plasmonic photoconversion enhancement mechanisms were tested for modulating photocurrent in PEC-based sensors using $\mathrm{Bi}_{3} \mathrm{FeMo}_{2} \mathrm{O}_{12}$ (BFMO) thin film semiconductor photoelectrodes and $\mathrm{Hg}^{2+}$ as a proof-of-concept analyte for detection. The possible plasmonic and non-plasmonic photoconversion enhancement mechanisms were controlled by choice of conjugated plasmonic nanoprobe between $\mathrm{Au}$ and $\mathrm{Au} @ \mathrm{SiO}_{2}$ coreshell nanoparticles with the BFMO. The conjugated Au NPs enhanced the BFMO thin film's PEC performance through a combination of plasmonic hot electron injection, PIRET, Fermi-level equilibration, and a non-plasmonic internal reflection within the BFMO caused by the conjugated $\mathrm{Au}$ NPs. The conjugated $\mathrm{Au} @ \mathrm{SiO}_{2} \mathrm{NPs}$ enhanced the BFMO thin film's PEC performance via PIRET and the non-plasmonic internal reflection within the BFMO caused by the $\mathrm{Au} @ \mathrm{SiO}_{2} \mathrm{NPs}$. 
A PEC sensor using the Au NPs as nanoprobes showed sensitivity and selectivity towards $\mathrm{Hg}^{2+}$ showing this PEC sensor design's potential.

In the third project presented, based on the comparison study of plasmonic and nonplasmonic photoconversion enhancement mechanisms with BFMO thin-film photoanodes, a PECbased immunosensor utilizing PIRET from Au NP-based nanoprobes conjugated to BFMO thinfilm photoanodes to modulate photoconversion of the BFMO is synthesized and studied using human immunoglobulin $\mathrm{G}$ (IGG) as a proof-of-concept analyte. The plasmonic Au NPs are conjugated in the presence of human IGG via antibody-antigen reactions. The resulting PIRETbased PEC immunosensor shows some sensitivity towards IGG detection. However, the sensitivity of the PIRET-based PEC immunosensor is limited due to the large separation distance $(\sim 10 \mathrm{~nm})$ between the plasmonic Au NPs the BFMO thin films from the antibody-antigen sandwich used for $\mathrm{Au}$ NP conjugation. As such, further work must focus on improving PIRET between the Au NP based nanoprobes and the BFMO thin film photoanodes. 


\section{Acknowledgements}

"Success is not final; failure is not fatal: It is the courage to continue that counts." - Winston Churchill

Of the many quotes on success that I have read, this one most completely summarizes my journey to complete this dissertation. However, my "courage" as Mr. Churchill put it has been built up and reinforced by many people along this journey that I would be remiss not to thank. Without them, it is very likely that this dissertation would not have been completed at all.

First, I would like to thank my parents, Jim and Amy, for instilling in me the required work ethic and self-reliance from a young age needed for these last seven years. I would like to Dr. Jiangtian Li and Scott Cushing for being my early research mentors. Without their early guidance, I would have been lost as I navigated a new world of scientific journals, conferences, and scientific research in general. The foundation that they built for me has been essential to completion of this journey. I would also like to thank the many graduate students and "post-docs" that I have worked with over the years. In particular, I would like to Fanke Meng, Peng Zheng, Dr. Xuefei Gao, Sujan Kasani, and Botong Liu. You have helped me so much from the beginning through the end of this journey.

I would like to thank my wife and colleague, Dr. Hui Yang. Without your guidance, being a springboard for my wacky ideas, and telling me when I have lost focus of the big picture, I genuinely do not think I could have completed this dissertation. Your support and refusal to let me give up has been the difference between courage and despair in this journey.

Last of all, I would like to thank Dr. Nianqiang Wu for being my research adviser for the last seven years. You have taught me a great deal about life as a researcher that I will not forget and have served as a positive influence in general. 


\section{Table of Contents}

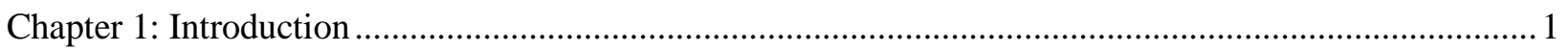

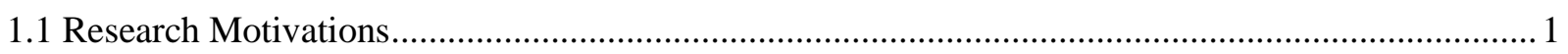

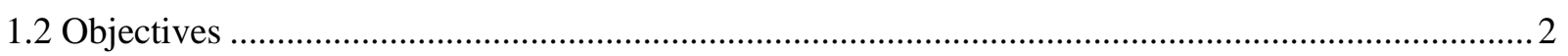

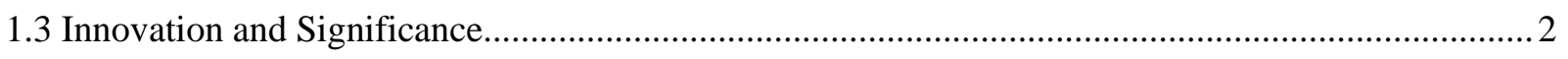

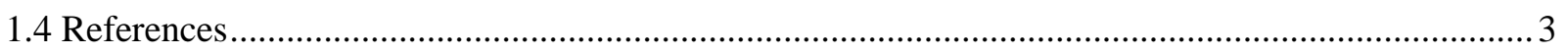

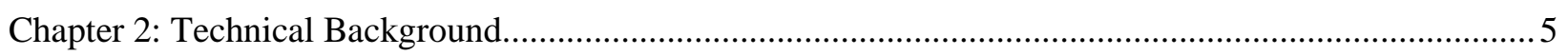

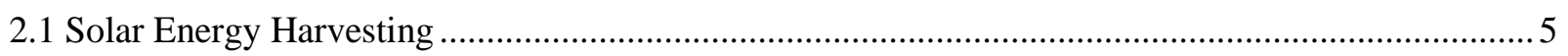

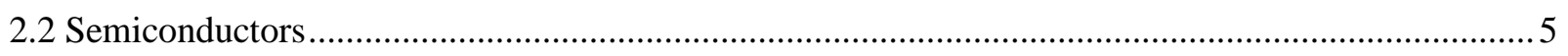

2.3 Photoelectrochemical (PEC) Cells and Photocatalysis ............................................................ 9

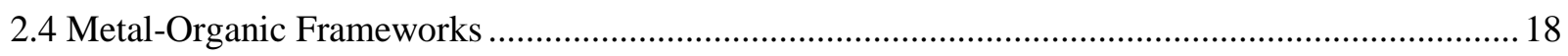

2.5 Plasmonics for Light Energy Harvesting Applications ...............................................................22

2.5.1 Plasmonic Effects for Modulating Semiconductor Photoconversion .......................................25

2.5.2 Non-Plasmonic Effects for Enhancing Photoelectrochemical Cell Performance .....................29

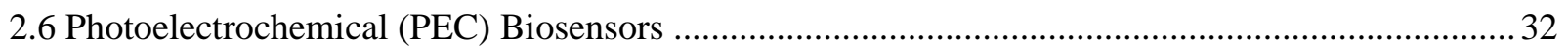

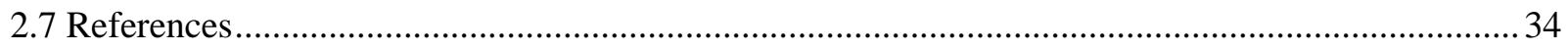

Chapter 3: Porphyrin-based Metal-Organic Framework Coated Titanium Dioxide Nanorod Array for Improved Photoelectrochemical Cell Performance ............................................................................ 46

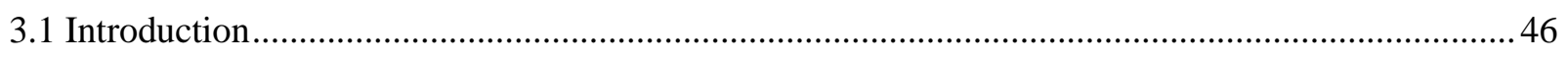

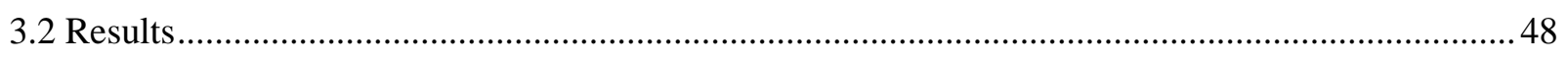

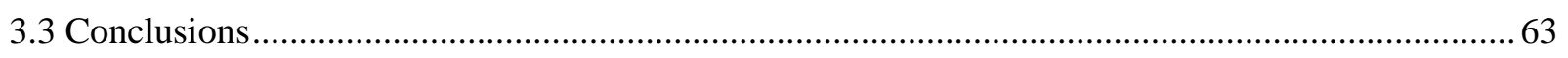

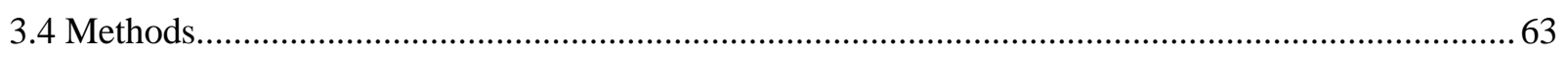

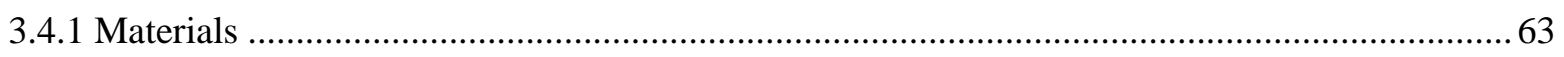

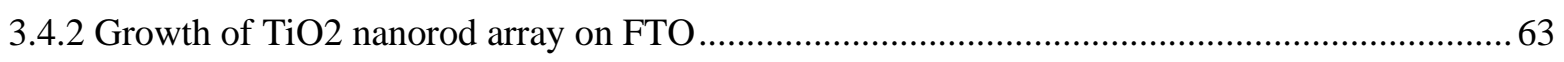

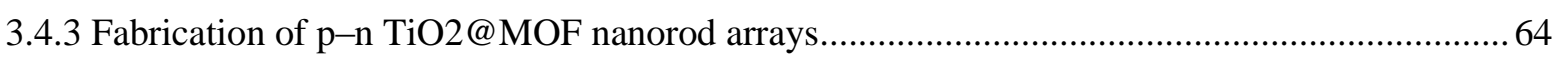

3.4.4 $\mathrm{Co}^{3+}$ functionalization of p-n TiO2@MOF nanorod array on FTO............................................64

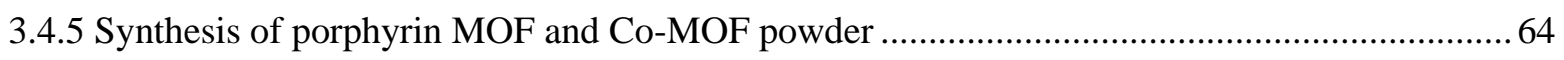

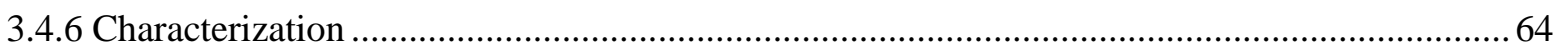

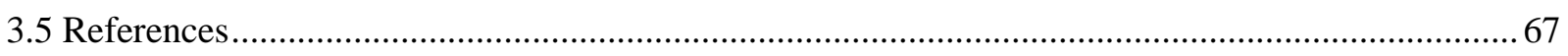

Chapter 4: Rational Design for a Photoelectrochemical (PEC) Sensor Utilizing Plasmonic Energy

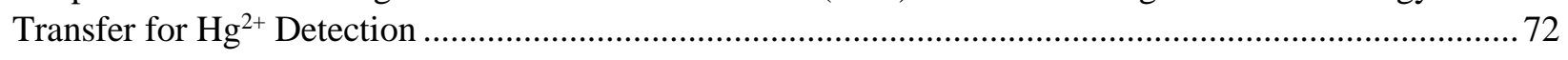

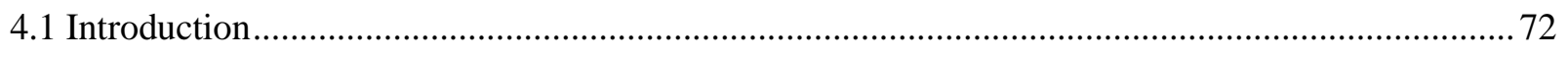

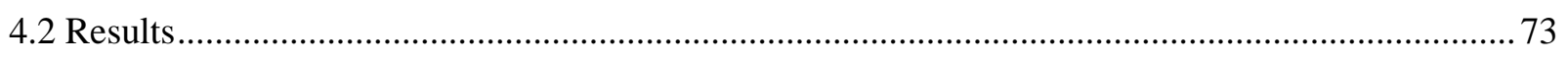

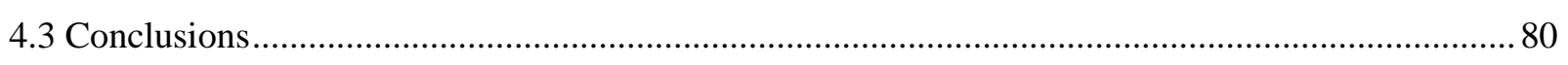

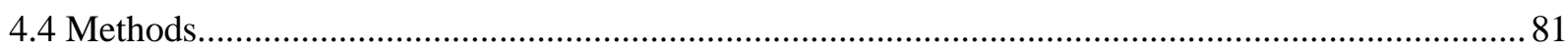




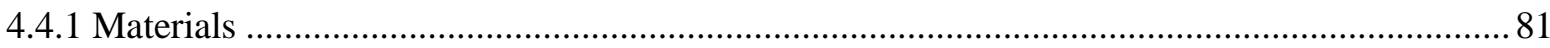

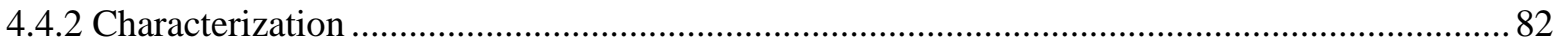

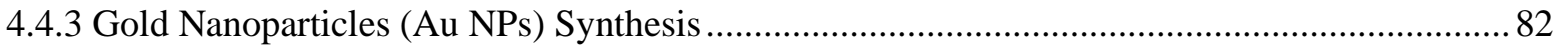

4.4.4 Gold@Silicon Dioxide Core@ Shell Nanoparticles (Au@ $\left.\mathrm{SiO}_{2} \mathrm{NPs}\right)$ Synthesis .......................82

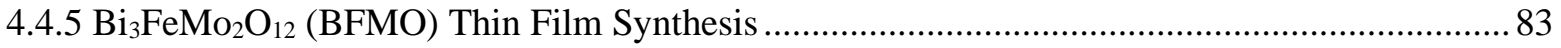

4.4.6 Labeling $\mathrm{Ag} @ \mathrm{SiO}_{2}$ and $\mathrm{Au} @ \mathrm{SiO}_{2}$ with Amine Group Linked Detection DNA Probe.............83

4.4.7 Labeling Au with Thiol-Group Linked Detection DNA Probe................................................ 83

4.4.8 Labeling Amine Group Linked Capture DNA Probe on BFMO Films .................................... 84

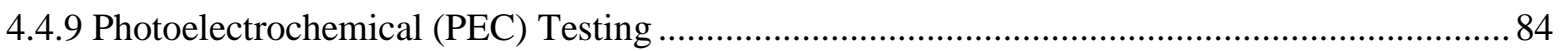

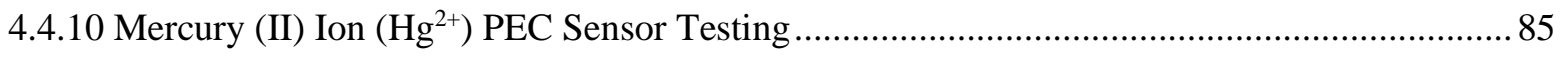

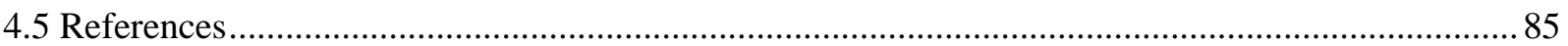

Chapter 5: Semiconductor Photoelectrochemical (PEC) Immunosensor Utilizing Plasmonic Energy

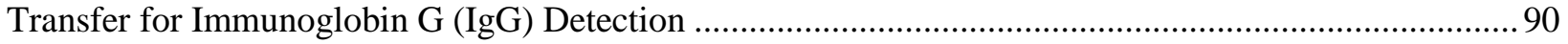

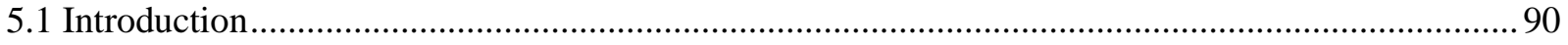

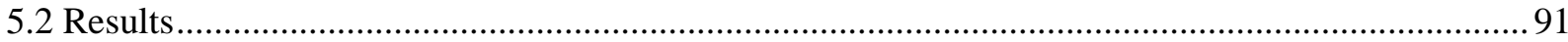

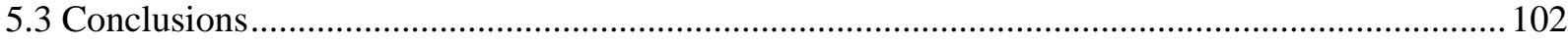

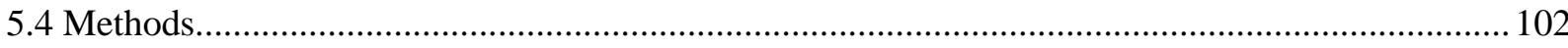

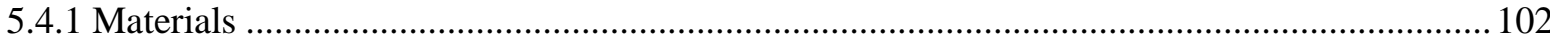

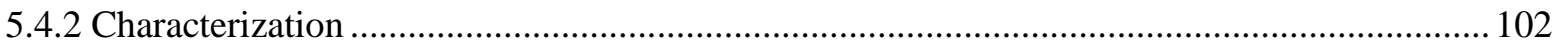

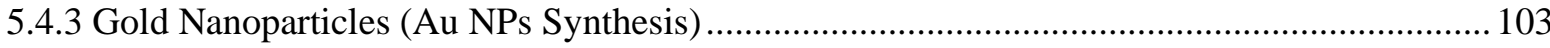

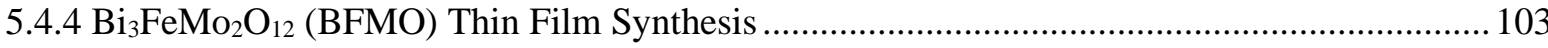

5.4.5 Labeling Anti-Human Immunoglobulin G (IGG) Capture Antibody onto BFMO Films........ 104

5.4.6 Labeling Anti-Human IGG Capture Antibody onto Au NPs .................................................. 104

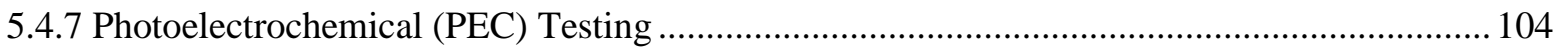

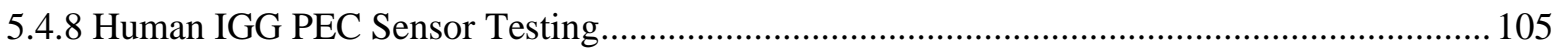

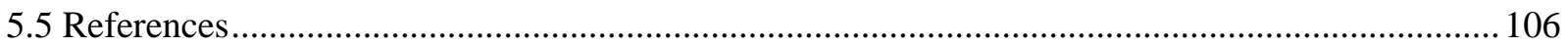

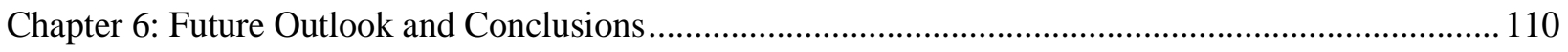

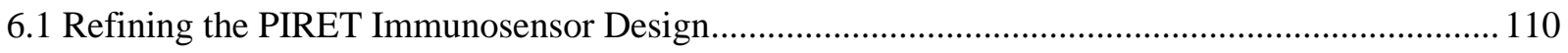

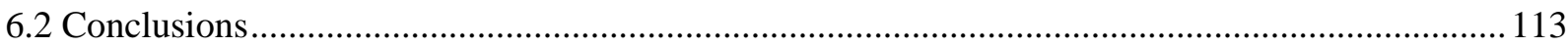

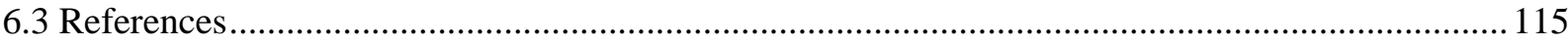

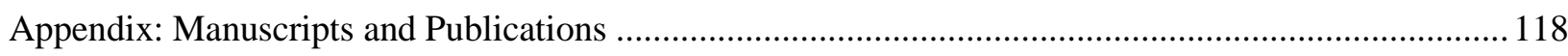

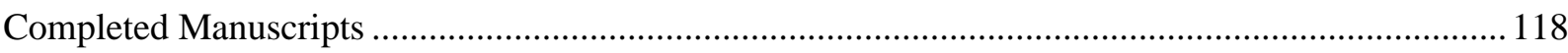

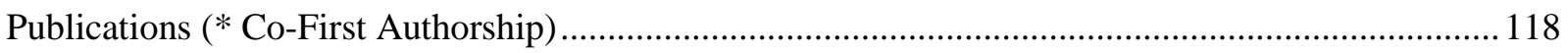




\section{Chapter 1: Introduction}

\subsection{Research Motivations}

Semiconductor devices are the basis of modern technology such as computers, cell phones, solid-state lighting, lasers, etc. ${ }^{[1]}$ One type of semiconductor device of interest is semiconductorbased photoconversion devices where incident light is converted into useful electrical signals. The useful electrical signals can be photoinduced changes to baseline currents, voltage, and resistance through the semiconductor. Semiconductor-based photoconversion devices can be used for several applications including light energy harvesting and conversion into more useful forms of energy such as heat, electricity or chemical energy, environmental remediation, and sensing of light, chemicals, biological substances.

Despite the usefulness of semiconductor photoconversion devices, these devices need further improvement to their photoconversion performance. Specific examples of the need for improved photoconversion performance are improved light energy harvesting and conversion efficiencies to electrical energy or chemical energy in photovoltaics and photoelectrochemical (PEC) cells respectively and improved photoconversion modulation response in the presence of analytes to increase the sensitivity of photoconversion-based sensors. While certain photovoltaic devices such as those based on silicon have become commercially viable, thin-film photovoltaics with commercially viable efficiencies are currently based on semiconductors composed of compounds containing toxic heavy metals such as cadmium $(\mathrm{Cd})$, lead $(\mathrm{Pb})$, tellurium $(\mathrm{Te})$, and selenium (Se) or extremely rare metals such as indium (In). ${ }^{[2,3]}$ As such, development of replacement semiconductors to replace heavy metal containing semiconductors is needed for these photoconversion devices.

In the case of PEC cells, commercial viability for solar energy harvesting applications has not been achieved due to materials challenges for finding semiconductors with sufficient light absorption, charge transport, and long-term chemical stability under operation to be efficient and stable enough for commercial application. ${ }^{[4,5]}$ As such, new semiconductor materials and materials combinations must be designed and tested to make PEC cells commercially viable for solar energy harvesting applications. However, applications that do not require high photoconversion device efficiencies but rather emphasize the ability to modulate the photoconversion performance of devices in response to the presence of some stimulus such as photoconversion-based sensors can utilize the PEC cell as a device architecture. In order for photoconversion-based sensors utilizing PEC cells as the device architecture to be commercially viable, new mechanisms for modulating photoconversion processes within semiconductor photoelectrodes in PEC cells must be developed. 


\subsection{Objectives}

The research presented in this dissertation shows three projects that focus on enhancing and/or controllably modulation photoconversion processes of semiconductor photoelectrodes within PEC cells. The objectives of the research presented within this dissertation are as follows:

- Design and test an ultra-thin porphyrin-based metal-organic framework overlayer onto metal-oxide based rutile titanium dioxide nanorod array photoelectrodes in order to enhance the performance towards solar water-splitting reactions.

- Compare the effectiveness of different photoconversion enhancement mechanisms from plasmonic metal-based nanoparticles evaluating mechanisms of both plasmonic and nonplasmonic origin for modulating photoconversion of semiconductor photoelectrodes for use as photoelectrochemical sensors.

- Practically apply plasmon-induced resonant energy transfer (PIRET) from plasmonic metal nanoparticles conjugated with semiconductor photoelectrodes for use in photoelectrochemical immunosensors for protein biomarker detection.

\subsection{Innovation and Significance}

In the first research presented, a porphyrin-based metal organic framework overlayer was coated onto a rutile titanium dioxide nanorod array for photoelectrochemical water-splitting. While metal-organic framework overlayers have been coated onto metal-oxide photoelectrodes including specifically rutile titanium dioxide nanorod arrays, there are no examples in literature of porphyrinbased metal-organic framework overlayers on metal oxide photoelectrodes. ${ }^{[6,7]}$ The research presented here demonstrates the potential utility of such porphyrin-based metal-organic frameworks as overlayers for improving the PEC water-splitting performance of metal-oxide photoelectrodes.

The second research project that evaluates different plasmonic and non-plasmonic photoconversion enhancement mechanisms from plasmonic metal nanoparticles for their use in modulating photoconversion within semiconductors. Most PEC sensors that utilize plasmonic energy transfer from plasmonic metals to modulate semiconductor photoconversion utilize almost exclusively the injection of hot electrons from plasmonic metal to semiconductor ${ }^{[8-10]}$ and Förster resonant energy transfer (FRET) to plasmonic metal nanoparticles from a semiconductor to modulate the photocurrent of semiconductor photoelectrodes. ${ }^{[11-13]}$ Few examples of PIRET-based photoconversion modulation in PEC sensors exist in literature and have no physics-based explanations of the resulting photoconversion modulation. As a result, no studies comparing different plasmonic and non-plasmonic photoconversion enhancement mechanisms for use in PEC sensors has not performed. The presented research remedies this lack of comparative studies while also providing a physics-based evaluation of the viability of different plasmonic and nonplasmonic photoconversion modulation mechanisms for use in PEC sensors. 
The last research project introduces a new concept for PEC sensors - the "turn-on" PIRETbased PEC immunosensor for protein biomarker detection. The non-radiative energy transfer nature of PIRET allows for it to occur over small separation distances between the plasmonic metal nanoparticle and semiconductor acceptor unlike hot electron injection processes that need direct contact between plasmonic metal nanoparticles and semiconductor. ${ }^{[14,15]}$ This ability to operate over short separation distances is necessary to overcome the separation distances induced by the antibody-antigen sandwich used for capturing analytes and secondary signal probes. While the ability to occur over small separation distances is also possible with FRET-based PEC sensors, FRET-based PEC sensors are typically "turn-off" devices that utilize quantum dots with long carrier dephasing times that are made of toxic heavy metals such as $\mathrm{Cd}, \mathrm{Pb}, \mathrm{Se}$, and Te. ${ }^{[1-13]} \mathrm{PIRET}$ does not require quantum dots to function effectively, allowing for non-toxic metal-oxide based semiconductors to be used as the semiconductor photoelectrode materials. In addition, the overall energy transfer efficiencies of PIRET are higher than hot electron injection, enabling more sensitivity with PIRET-based PEC sensors. ${ }^{[14,15]}$ As such, the presented research lays a foundation for designing PEC-based sensors utilizing PIRET to modulate photoconversion within semiconductor photoelectrodes.

\subsection{References}

[1] Muller, R. S.; Kamins, T. I.; Chan, M. Semiconductor Electronics. Device Electronics for Integrated Circuits, John Wiley \& Sons: New York, 2003; pp 2-55.

[2] Green, M. A.; Hishikawa, Y.; Dunlop, E. D.; Levi, D. H.; Hohl-Ebinger, J.; Yoshita, M.; HoBaillie, A. W. Y. Solar cell efficiency tables (Version 53). Prog. Photovolt. Res. Appl., 2019, 27, 3-12.

[3] Wallace, S. K.; Mitzi, D. B.; Walsh, A. The steady rise of kesterite solar cells. ACS Ener. Lett., 2017, 2, 776-779.

[4] Li, J.; Wu, N. Semiconductor-based photocatalysts and photoelectrochemical cells for solar fuel generation: a review. Catal. Sci. Technol., 2015, 5, 1360-1384.

[5] Sivula, K.; van de Krol, R. Semiconducting materials for photoelectrochemical energy conversion. Nat. Rev. Mater., 2016, 1, 15010.

[6] Zhang, L.; Cui, P.; Yang, H.; Chen, J.; Xiao, F.; Guo, Y.; Liu, Y.; Zhang, W.; Huo, F.; Liu, B. Metal-organic frameworks as promising photosensitizers for photoelectrochemical water splitting. Adv. Sci., 2016, 3, 1500243.

[7] Jiao, Z.; Zheng, J.; Feng, C.; Wang, Z.; Wang, X.; Lu, G.; Bi, Y. Fe/W co-doped BiVO4 photoanodes with a metal-organic framework cocatalyst for improved photoelectrochemical stability and activity. ChemSusChem, 2016, 9, 2824-2831. 
[8] Shi, Y.; Zhang, Q.; Zhai, T.-T.; Zhou, Y.; Yang, D.-R.; Wang, F.-B.; Xia, X.-H. Localized surface plasmon resonance enhanced label-free photoelectrochemical immunoassay by Au-MoS 2 nanohybrid. Electrochim. Acta, 2018, 271, 361-369.

[9] Da, P.; Li, W.; Lin, X.; Wang, Y.; Tang, J.; Zheng, G. Surface plasmon resonance enhanced real time photoelectrochemical protein sensing by gold nanoparticle-decorated $\mathrm{TiO}_{2}$ nanowires. Anal. Chem., 2014, 86, 6633-6639.

[10] Qiao, Y.; Li, J.; Li, H.; Fang, H.; Fan, D.; Wang, W. A label-free photoelectrochemical aptasensor for bisphenol A based on surface plasmon resonance of gold nanoparticlesensitized ZnO nanopencils. Biosens. Bioelectron., 2016, 86, 315-320.

[11] Zhao, W.-W.; Yu, P.-P.; Shan, Y.; Wang, J.; Xu, J.-J.; Chen, H.-Y. Exciton-plasmon interactions between $\mathrm{CdS}$ quantum dots and $\mathrm{Ag}$ nanoparticles in photoelectrochemical system and its biosensing application. Anal. Chem., 2012, 84, 5892-5897.

[12] Han, D.-M.; Jiang, L.-Y.; Tang, W.-Y.; Xu, J.-J.; Chen, H.-Y. Photoelectrochemical determination of inorganic mercury ions based on energy transfer between CdS quantum dots and Au nanoparticles. Electrochem. Commun., 2015, 51, 72-75.

[13] Wang, Y.; Yu, X.; Ye, X.; Wu, K.; Wu, T.; Li, C. Resonance energy transfer between $\mathrm{ZnCdHgSe} \mathrm{quantum} \mathrm{dots} \mathrm{and} \mathrm{gold} \mathrm{nanorods} \mathrm{enhancing} \mathrm{photoelectrochemical}$ immunosensing of prostate specific antigen. Anal. Chim. Acta, 2016, 943, 106-113.

[14] Li, J.; Cushing, S. K.; Meng, F.; Senty, T. R.; Bristow, A. D.; Wu, N. Plasmon-induced resonance energy transfer for solar energy conversion. Nat. Photon., 2015, 9, 601-607.

[15] Wu, N. Plasmonic metal-semiconductor photocatalysts and photoelectrochemical cells: a review. Nanoscale, 2018, 10, 2679-2696. 


\section{Chapter 2: Technical Background}

\subsection{Solar Energy Harvesting}

The amount of incident sunlight reaching the Earth's surface each year is enough to provide 7500 times the world's current yearly energy demands. ${ }^{[1]}$ There are various methods to harvest the energy from solar radiation and convert it into other forms of energy that are useful within society such as heat and electricity. Such methods include photovoltaics, solar-thermal technology, photosynthetic microbe-based biofuels, and photoelectrochemical (PEC) cells or photocatalysis. Of the listed techniques, photovoltaics is the most commonly used solar energy harvesting technology. However, thin-film photovoltaics that generate electricity from photoexcited charge carriers are not efficient or cheap enough to manufacture to be currently economically competitive with fossil fuels on a widescale.

For the purposes of this dissertation, research focus will be spent on solar energy harvesting using semiconductor photoconversion from photoelectrochemical (PEC) cells and photocatalysis only. The basic physics of semiconductors and semiconductor materials in general must be discussed before discussing PEC cells and photocatalysis. In addition, discussion on the concept of the semiconductor $\mathrm{p}-\mathrm{n}$ junction is necessary for context on the research within Chapter 3 of this dissertation.

\subsection{Semiconductors}

Semiconductors are a class of materials that is the basis of most electronics technology since the 1950's due to the advent of single crystal silicon manufacturing. ${ }^{[2]}$ The main property that distinguishes semiconductors from metals and insulator materials is the small finite energy gap, known as a bandgap, between the valence band and conduction band of the material. ${ }^{[3-4]}$ The valence band and conduction bands may also be called the highest occupied molecular orbital (HOMO) and lowest unoccupied molecular orbital (LUMO) respectively particularly within the chemistry community. Metals have overlapping valence and conduction bands while insulators have extremely large bandgaps that significantly limit charge carrier movement through the material. There is some conflict as to the range of bandgap energies that constitute a semiconductor versus an insulator. Traditional definitions state semiconductors have bandgaps between $1-2$ $\mathrm{eV}{ }^{[3]}$ This definition has been relaxed somewhat in recent years to accommodate wider bandgap materials that are used for optical devices. For the purposes of this dissertation, semiconductors will be treated as materials with bandgaps within the energy range of the solar spectrum $(1.0-4.0$ $\mathrm{eV})$.

For solar or light energy harvesting applications, the optical and charge transport properties of semiconductors are of the most interest. Semiconductors have several unique properties due to 
their small bandgaps. Starting with optical properties, a semiconductor can absorb light photons with energy greater than or equal than its bandgap $\left(h v \geq E_{g}\right){ }^{[9,10]}$ The incident light energy results in excitation of an electron from the ground state in the valence band to an excited state within the conduction band. The resulting electron vacancy in the valence band, typically considered a quasiparticle called a "hole", is also a charge carrier that can move through the semiconductor. ${ }^{[9-}$ ${ }^{11]}$ There are two different types of semiconductor bandgap types as depicted in Figure 2.1.
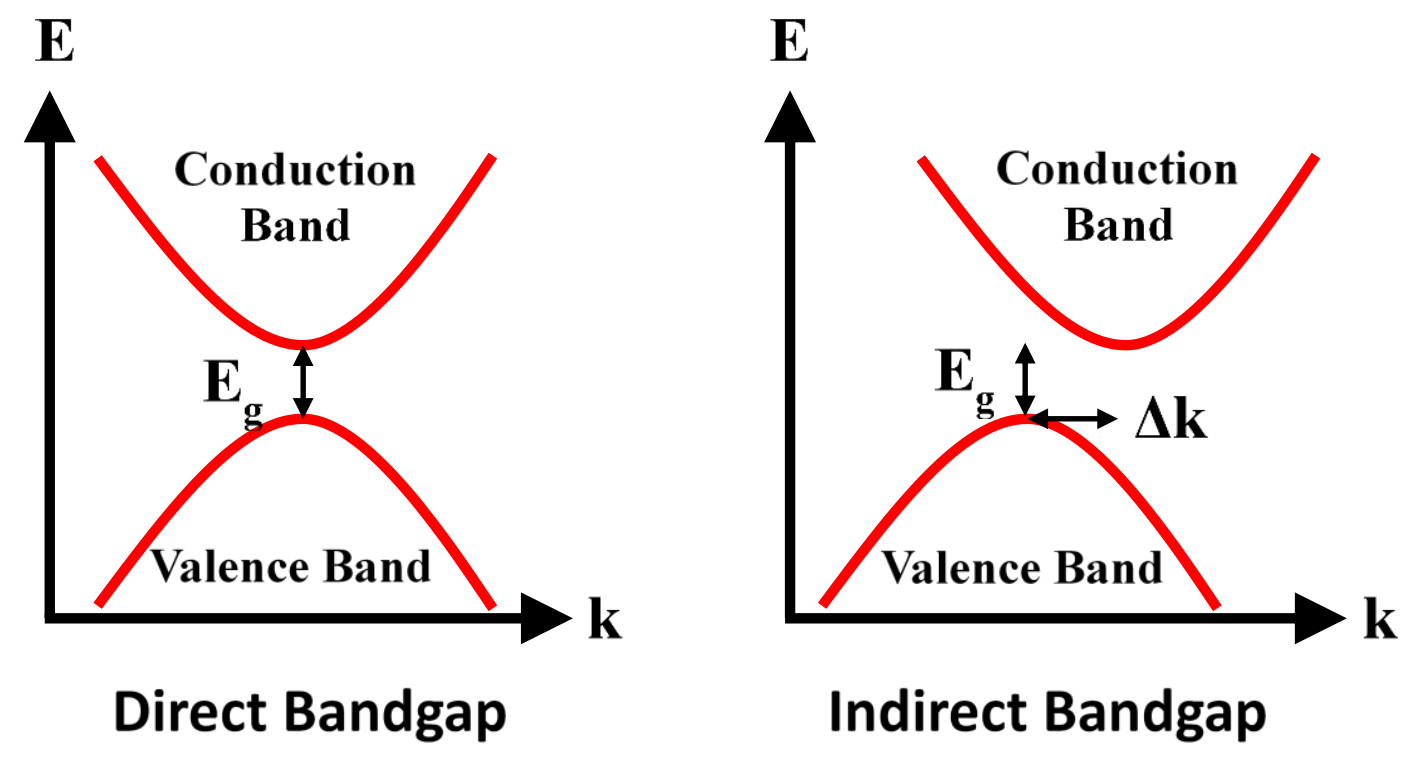

Figure 2.1: Band diagrams ( $E-k$ diagrams) illustrating the types of semiconductor bandgaps.

The first type of semiconductor bandgap is a "direct" bandgap. A direct bandgap has valence and conduction bands with matching crystal momenta $(k) \cdot{ }^{[3,5]}$ The second type of semiconductor bandgap is an "indirect" bandgap where the valence and conduction bands have mismatching crystal momenta with a momenta difference of $\Delta k$. The mismatched valence band and conduction band momenta of an indirect bandgap requires an additional source or sink of momentum such as a phonon to facilitate an electron transition between valence and conduction bands. ${ }^{[3,5]}$ As such, absorption coefficients are significantly lower for indirect bandgap semiconductors than direct bandgap semiconductors that do not require an additional momentum source or sink to facilitate an electronic transition. ${ }^{[3,6]}$

Electrons can have two competing possibilities happen after they are photoexcited from the valence band to the conduction band in a semiconductor. The excited electron can return its ground state by recombining with a hole with the excess energy released through either radiative, non-radiative or Auger recombination. ${ }^{[5-7]}$ The other possibility is that the electron or hole can be extracted from the semiconductor through different ways (e.g. transfer to an external circuit, transfer to a chemical species, etc.) before recombination takes place. Determining whether excited electrons and holes recombine or are extracted from a semiconductor depends on a variety of semiconductor material properties such as excited carrier lifetimes, electron and hole 
conductivities, electron and hole mobilities, etc. in addition to the interface or surrounding media around the semiconductor. ${ }^{[5-7]}$

One of the most important properties of a semiconductor is whether negatively charged electrons or positively charged holes are the majority or dominant charge carrier population within the semiconductor. A n-type semiconductor with electrons as the dominant carrier has a significant non-negligible population of electrons within the conduction band at equilibrium conditions. This results in a Fermi level $\left(E_{F}\right)$, defined as the energy at which the probability of finding a free electron is 50 percent, that is close to the conduction band. ${ }^{[3-5]}$ Similarly, a p-type semiconductor with holes as the dominant carrier has a negligible free electron population within the conduction band at equilibrium. ${ }^{[3-5]}$ The lack of free electrons within the conduction band results in a Fermi level close to valence band. Figure 2.2 illustrates this difference. To alter the type of dominant charge carrier or other electrical or optical properties of a semiconductor, introduction of dopant ions into the semiconductor may be performed. A classic example of this is phosphorous or aluminum doping of silicon to form n-type or p-type silicon with high free carrier densities respectively. ${ }^{[8]}$

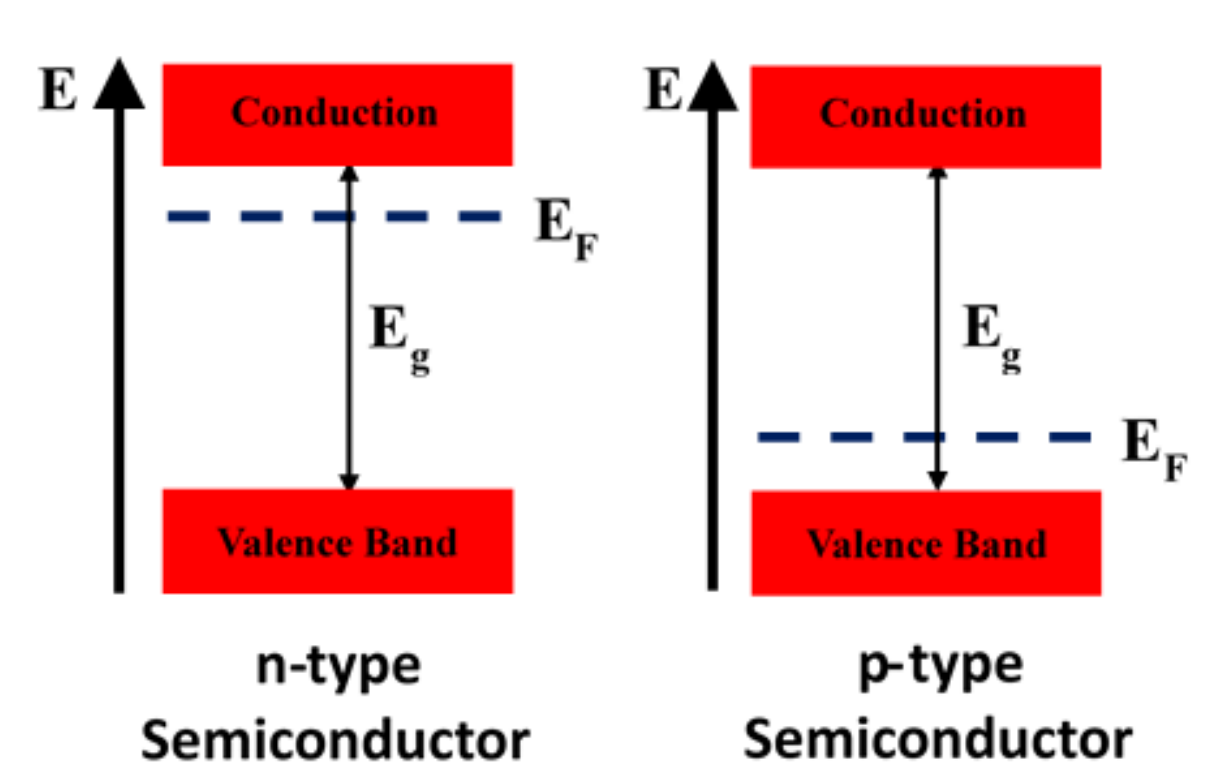

Figure 2.1: Types of semiconductors on basis of majority charge carrier.

Additionally, the electrical conductivity of a semiconductor increases with temperature and light illumination (known as photoconductivity). ${ }^{[4]}$ The increase in a semiconductor's electrical conductivity results from charge carrier excitation from valence band or defect states to conduction band either thermally or by light absorption respectively. These excited charges in the conduction band are more easily transported through the semiconductor than unexcited charge carriers in the valence band due to the delocalized nature of electrons within a semiconductor's conduction band. However, conductivity is compromised at elevated temperatures due free carrier scattering from collisions with phonons and ion impurities. As such, temperature control is a necessity for semiconductor devices. ${ }^{[4]}$ 
The "p-n junction" is a layered combination of n-type and p-type semiconductors in direct contact with each other. Figure 2.3 illustrates the electronic structure of the semiconductors before and after formation of a p-n homojunction. The resulting contact between n-type and p-type semiconductors causes equilibrium electron and hole populations to form within the semiconductors, as denoted by a constant Fermi level $\left(E_{F}\right)$ across both semiconductors). ${ }^{[7,9,10]}$ To reach this equilibrium, a significant migration of free electrons from n-type semiconductor to $\mathrm{p}$ type semiconductor within part of the n-type semiconductor near the contact with the p-type semiconductor. A similar migration of free holes from the p-type semiconductor to the n-type semiconductor near the contact between semiconductors also occurs. The migration of free electrons and holes from the n-type and p-type semiconductors and introduction of those migrated electrons and holes into the p-type and n-type semiconductors respectively results in the region immediately surrounding the $\mathrm{p}-\mathrm{n}$ junction having negligible free charge carriers. This region is colloquially referred to as a "depletion zone". ${ }^{[7,9,10]}$ The width of this depletion zone depends on the charge carrier densities of the n-type and p-type semiconductors with higher carrier densities resulting in a shorter depletion zone width. The formation of the depletion region within the p-n junction also results in the formation of a built-in electric field $\left(\vec{E}_{b i}\right)$ and built-in voltage $\left(V_{b i}\right)$ with magnitude related to the difference in Fermi levels in the p-type and n-type semiconductor.

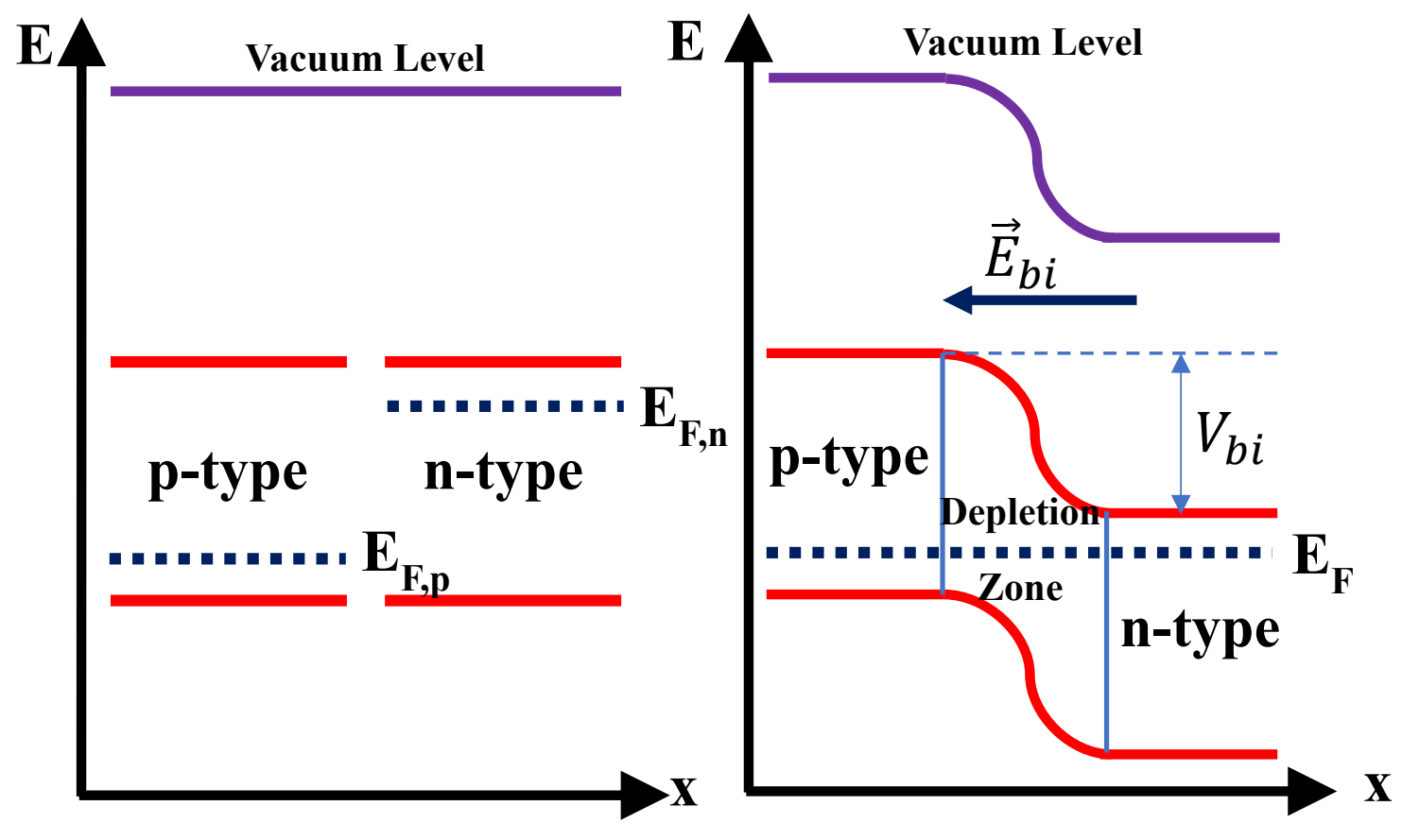

Equilibrium Before Contact

Equilibrium with p-n Junction

Figure 2.2: Equilibrium energy band diagram of a p-n homojunction as a function of depth (x) within semiconductors.

Once a semiconductor $\mathrm{p}-\mathrm{n}$ junction is illuminated, electrons are excited from valence band to conduction band. This results in a new non-equilibrium carrier distribution throughout the $\mathrm{p}-\mathrm{n}$ 
junction. ${ }^{[6,7,11]}$ The traditional approach to evaluate this non-equilibrium is treat electrons and holes separately by giving both electrons and holes their own Fermi levels (called a quasi-Fermi level) that reflect the non-equilibrium carrier distributions within the p-n junction. The nonequilibrium carrier distribution results in charge carriers moving throughout the $\mathrm{p}-\mathrm{n}$ junction to reach a new equilibrium condition. ${ }^{[6,7,11]}$ The flow of charge carriers is a photocurrent that can be used to generate electricity in the case of photovoltaics or produce chemical products in the case of PEC cells or photocatalysts. ${ }^{[1]}$ The amount of current produced by a photovoltaic device is related to the balance of photoexcited electron and photoexcited hole generation with photoexcited electron recombination with photoexcited holes. Several factors affect the both photogeneration rates and recombination rates. These include but are not limited to the absorption coefficient of the semiconductor, the intensity of light-illumination with the semiconductor device, and charge transport properties (i.e. electron/hole mobilities, electron/hole diffusion lengths). The separation between quasi-Fermi levels for electrons and holes results in a photovoltage being generated. In the case of p-n junction, the upper limit for this photovoltage is ultimately the original built-in voltage from the formation of the semiconductor $\mathrm{p}-\mathrm{n}$ junction. Thus, the upper limit to photovoltage which occurs at an open-circuit condition with no flowing current, is directly tied to selection of materials used to create the semiconductor p-n junction device. ${ }^{[1]}$

\subsection{Photoelectrochemical (PEC) Cells and Photocatalysis}

The main solar energy harvesting research topic that utilizes semiconductor photoconversion devices discussed in this dissertation is utilization of photocatalysis or photoelectrochemical (PEC) cells. ${ }^{[12-32]}$ Unlike photovoltaics that directly generate electricity using photoexcited charge carriers from semiconductors, semiconductor-based photocatalysts and photoelectrochemical cells directly use photoexcited charge carriers to drive reduction-oxidation (redox) reactions on the surface of a semiconductor immersed in an electrolyte solution. The main advantage to photocatalysis and PEC cells in comparison to photovoltaic devices for solar energy harvesting is that solar energy is directly stored in the form of chemical bonds of photocatalytic reaction products. The reaction products could be used for either chemical feedstock for manufacturing or for power generation. Figure 2.4 shows the basic operating principles for photocatalysts and photoelectrochemical cells. The working principle illustrated applies regardless of the chemical reaction that is being performed on the photocatalyst/photoelectrode. Common photocatalytic reactions that are heavily researched are solar water-splitting into $\mathrm{H}_{2}$ and $\mathrm{O}_{2}$ gas ${ }^{[12-}$ ${ }^{28]}, \mathrm{CO}_{2}$ reduction in the presence of protons into hydrocarbons ${ }^{[29-31]}$, and fixation of atmospheric nitrogen $\left(\mathrm{N}_{2}\right)$ into nitrogen containing compounds such as ammonia $\left(\mathrm{NH}_{3}\right)^{[32]}$.

As can be seen from Figure 2.4 below, sunlight illumination and subsequent photoexcited charge generation occurs within the bulk of the semiconductor photocatalyst (Figure 2.4a). Both electrons and holes can migrate to the surface of the photocatalyst with a barrier for transport to the semiconductor/electrolyte interface for the photocatalyst's majority charge carriers due to band bending that occurs due to Fermi level equilibration between the semiconductor photocatalyst and the redox potential of the electrolyte solution used that favors minority charge transport to the 
semiconductor/electrolyte interface. (Figure 2.4b). Once the photoexcited electrons and holes reach the surface, they can be consumed in redox reactions that produce potentially useful products.
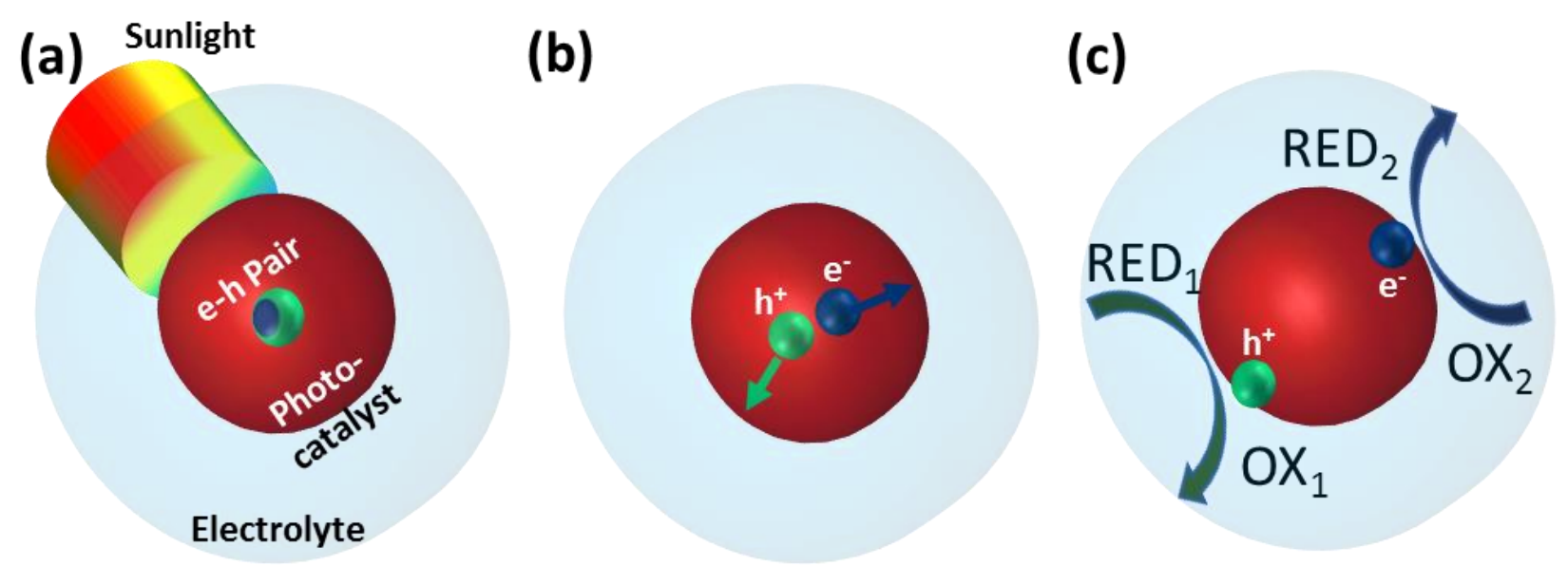

Figure 2.3: Basic operating principles for particle-based photocatalysts; (a) excited electron-hole (e-h) pair generated upon light excitation; (b) Photoexcited electrons ( $\left.\mathrm{e}^{-}\right)$and holes $\left(\mathrm{h}^{+}\right)$separate and migrate to photocatalyst surface; (c) Photoexcited electrons and holes drive reduction and oxidation reactions at photocatalyst surface using reduced (RED) and oxidized (OX) forms of chemicals as reaction reactants and products.

In the case of photoelectrodes in a PEC cell (Figure 2.5), majority carriers travel towards back contact of the photoelectrode to be transported to the counter electrode used to complete the circuit in the PEC cell. At the semiconductor/electrolyte interface, accumulated charge carriers, holes and electrons for n-type photoanodes (Figure 2.5a) and p-type photocathodes (Figure 2.5b) respectively, either are injected into chemical species in the electrolyte or recombine with accumulated charge carrier of opposite charge. In both the case of particulate photocatalysts or semiconductor photoelectrodes, both electrons and holes must be extracted from the photocatalyst material for efficient operation or significant recombination due to an excess of the unextracted carrier will limit the efficiency of the process. 

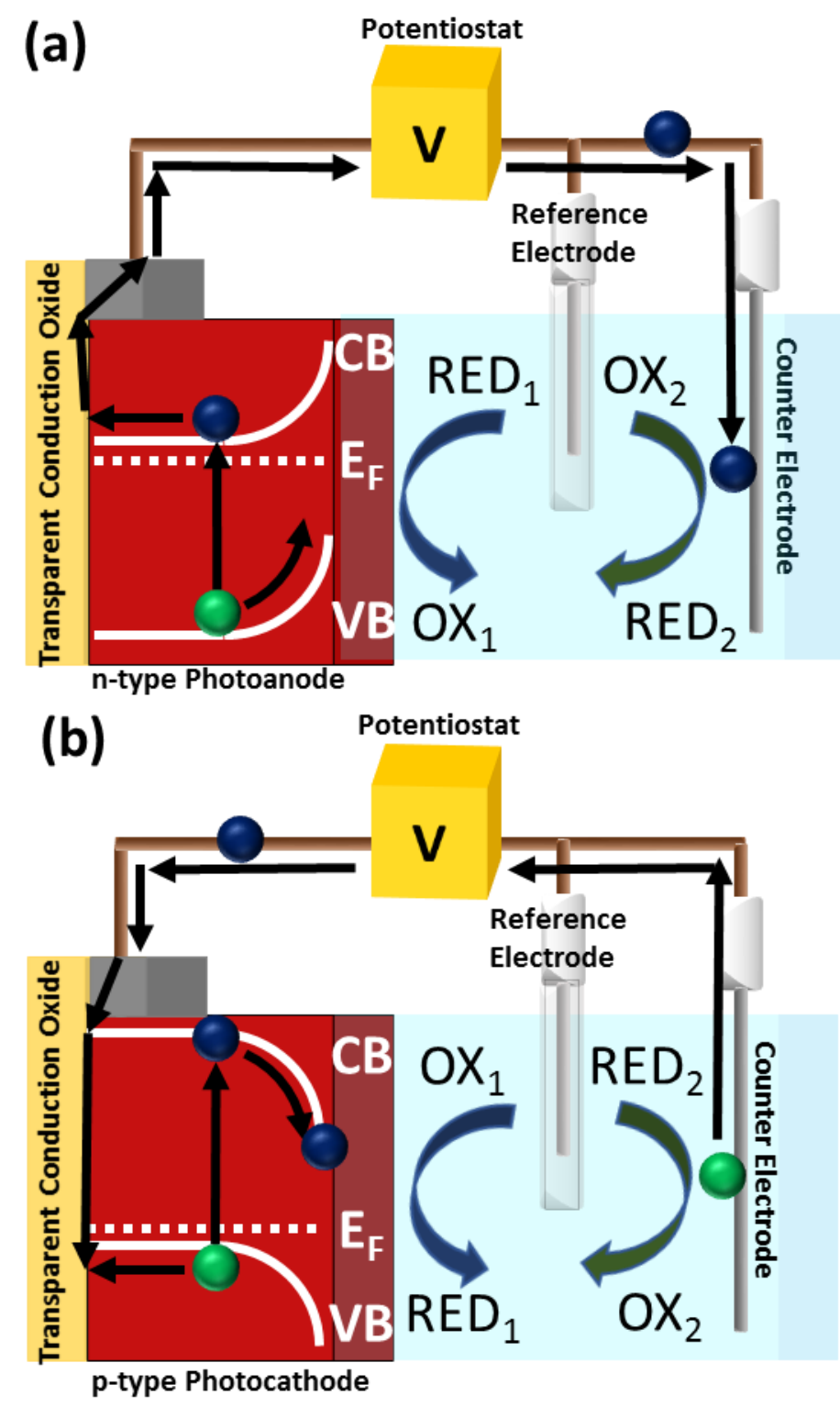

Figure 2.5: Basic operating principles for (A) photocatalysts and (B) n-type photoanodes in electrolytes; Blue circles: electrons; Gray circles: holes; Red circles or rectangles: photocatalyst or photoanode material; Tan circle: potentiostat; Gray line: counter electrode.

The performance of semiconductor-based photoconversion devices such as photovoltaics, photocatalysts, photoelectrochemical cells is tied directly to three processes - (1) light absorption by the device, (2) photoexcited charge transport within the device, and (3) injection of photoexcited charge to chemical species (like in photocatalysts and PEC cells) or an external circuit (in the case of photovoltaics). Photocurrent from light harvesting devices can be expressed as in Equation 2.1: [19]

$$
J_{p h}=J_{A b s} \cdot P_{\text {Sep }} \cdot P_{\text {Inject }}
$$


where $J_{p h}$ is the measured photocurrent of a device, $J_{A b s}$ is the theoretical photocurrent a device should have based on its light absorption spectrum assuming all photoexcited charge is harvested, $P_{S e p}$ is the efficiency for photoexcited charge separation and transport within a device without recombination, and $P_{\text {Inject }}$ is the efficiency for photoexcited charge injection to surface adsorbed chemical species as in the case of photocatalysts and photoelectrodes in PEC cells or an external circuit as in the case of photovoltaics. As such, improving photoconversion efficiencies in semiconductor-based light harvesting devices can only be done by improving these three basic processes.

Based on the processes just described in Figure 2.4, Figure 2.5, and Equation 2.1, there are several properties that ideal photocatalyst and photoelectrode materials must have. First, the photocatalyst/photoelectrode material should have a bandgap well matched to the solar spectrum to increase $J_{a b s}$ while still having sufficient energy to overcome overpotentials associated with driving the desired chemical reaction. To use a photocatalyst/photoelectrode based on a single material designed for driving solar water-splitting as an example, the ideal bandgap for a single photocatalyst/photoelectrode system is $1.9-2.2 \mathrm{eV}$ due to reaction energetics and overpotentials associated with water-splitting ${ }^{[13]}$ with recent studies placing realistic efficiencies for single material junction photoelectrodes at $15.6 \%$ for $2.05 \mathrm{eV}$ bandgap material ${ }^{[22]}$.

Second, the photocatalyst/photoelectrode should have sufficient charge transport properties (i.e. electron and hole mobilities and diffusion lengths) to enable minority carriers to diffuse from the bulk to the semiconductor/electrolyte interface while the material is still optically thick enough to absorb most incoming sunlight. This balance between charge transport and optical absorption lengths is one of the major challenges in photocatalysis/PEC cell materials research and highlights that the three underlying processes in semiconductor-based photoconversion are linked. ${ }^{[13-19]}$

Third, the photocatalyst/photoelectrode material must have good catalytic ability (i.e. fast charge transfer kinetics) for driving the desired photocatalytic/photoelectrochemical reaction. If the photocatalyst/photoelectrode has low catalytic activity for driving a certain reaction, excited charge carriers at the semiconductor/electrolyte interface are more likely to recombine than react with chemical species - resulting in a low $P_{\text {Inject }}$ and overall poor photocurrent for the photocatalyst/photoelectrochemical device. ${ }^{[16-19]}$

Lastly, the photocatalyst/photoelectrode material should be photostable (i.e stable during light-illumination) while in the electrolyte solution used for photocatalytic/photoelectrochemical reactions. The main causes of photodegradation are possible redox reactions resulting in irreversible changes to the photocatalyst/photoelectrode material being more thermodynamically and kinetically favorable to the desired photocatalytic/photoelectrochemical reaction. ${ }^{[13-16,18,19]} \mathrm{A}$ classic example of this tungsten (VI) oxide $\left(\mathrm{WO}_{3}\right)$ being stable in electrolytes with $\mathrm{pH} \leq 4 .{ }^{[23]}$ This is due to $\mathrm{WO}_{3}$ conversion to $\mathrm{WO}_{4}{ }^{2-}$ in the presence of $\mathrm{OH}^{-}$ions being a more favorable redox reaction than solar water-splitting to $\mathrm{O}_{2}$ gas and protons. By keeping the $\mathrm{pH} \leq 4$, water-oxidation becomes more kinetically favorable due to a lack of $\mathrm{OH}$ - needed for $\mathrm{WO}_{4}{ }^{2-}$ ion formation with $\mathrm{WO}_{3}$ - thus keeping $\mathrm{WO}_{3}$ photostable for solar water-splitting. As such, photostability of photocatalyst/photoelectrode materials and good catalytic activity towards a given reaction can be 
directly related. This is an additional design consideration with photocatalyst/PEC cell systems. In the case of photoelectrodes in a PEC cell, excess majority carriers can be extracted into an external circuit and routed to a counter electrode carrying out a complementary half-reaction to complete the circuit. In the case of particulate photocatalysts or photoelectrodes with poor majority carrier transport, the photocatalyst or photoelectrode must be able to drive two separate reactions using both electrons and holes to prevent performance-limiting recombination or even photodegradation of the photocatalyst/photoelectrode system. To solve this issue, different approaches have been used.

The first approach to reduce charge carrier recombination and is to use a very easily oxidized or reduced chemical (commonly referred to as a sacrificial reagent) with redox potential well matched to the energy of the charge carrier that needs to be extracted. Common examples of this are hole scavengers such as hydrogen peroxide $\left(\mathrm{H}_{2} \mathrm{O}_{2}\right)$, triethanolamine (TEA), and sodium sulfite $\left(\mathrm{Na}_{2} \mathrm{SO}_{3}\right)^{[33]}$ or electron scavengers like $\mathrm{Ag}^{+}$and $\mathrm{Fe}^{3+} \cdot{ }^{[34]}$ However, since the sacrificial reagent is irreversibly consumed during the reaction, a steady supply of sacrificial reagent is needed for continuous operation. This can add to the cost of a photocatalyst system unless the products from sacrificial reagent consumption are more economically valuable than the original sacrificial reagent. ${ }^{[23,36,37]} \mathrm{A}$ good example of this case is the use of 5-hydroxymethlfurfural (HMF) as a hole scavenger to produce valuable 2,5-furandicarboxylic acid (FDCA). ${ }^{[36,45]}$ An additional problem with this approach is so called "current-doubling" that can result from consumed sacrificial reagent products undergoing further chemical reactions that result in charge carrier injection from chemical to photocatalyst/photoelectrode. ${ }^{[3]}$ The additionally injected majority charge carrier artificially increases the measured current without a change in the overall number of minority carriers being consumed. A good example of this is methanol oxidation $\left(\mathrm{CH}_{3} \mathrm{OH}\right)$ to formaldehyde $(\mathrm{HCHO})$ without the presence of $\mathrm{O}_{2}$ in photocatalyst/PEC systems. To further oxidize the first reaction intermediate $\left(\mathrm{CH}_{2} \mathrm{OH}^{*}\right)$ to $\mathrm{HCHO}$ without $\mathrm{O}_{2}$ as an oxidizer, the $\mathrm{CH}_{2} \mathrm{OH}^{*}$ injects an electron into the photoelectrode and releases a proton to complete $\mathrm{HCHO}$ formation. This subsequently obfuscates how effectively a photocatalyst/photoelectrode system is performing since an additional charge carrier from an outside source is injected into the system. As such, sacrificial reagents are not a preferred way to improve photocatalyst/PEC cell performance if other alternatives are available.

The second approach to reduce charge carrier recombination and photostability is to use a redox mediator in conjunction with two photoelectrodes or a photoelectrode and electrocatalyst designed to drive separate but complementary reduction or oxidation reactions. An increasingly popular example of this approach is the use of electron-coupled proton buffers (ECPBs) such as phosphomolybdic acid $\left(\mathrm{H}_{3} \mathrm{PMo}_{12} \mathrm{O}_{40}\right)^{[37,38]}$, silicotungstic acid $\left(\mathrm{H}_{4}\left[\mathrm{SiW}_{12} \mathrm{O}_{40}\right)^{[39]}\right.$, or quinones ${ }^{[40]}$, that can be reversible oxidized and reduced using the majority carrier from the photoelectrode on a third electrocatalyst. This approach does not require a constant supply of available sacrificial reagent for consumption and do not suffer from current-doubling. However, current ECPBs are expensive chemicals that would significant increase the cost of a photoelectrochemical systems. Nonetheless, this approach may be more economically viable if current ECPBs manufacturing costs decrease or more commonly available chemicals are discovered to be viable ECPBs. 
The third and most commonly utilized approach to reduce charge carrier recombination and is the use of co-catalyst materials for driving the desired redox reaction. These co-catalyst materials on the surface of the photocatalyst/photoelectrode rapidly extract the minority charge carrier to be used in driving the desired redox reaction out of the photocatalyst/photoelectrode and then inject the minority charge carriers into chemical species in the electrolyte. By rapidly extracting the minority carriers out of the semiconductor photocatalyst/photoelectrode, recombination with majority carriers at the surface is prevented. In the case of water-oxidation, examples of common co-catalyst materials are cobalt phosphate $(\mathrm{Co}-\mathrm{Pi})^{[16,18,41]}$, nickel and iron oxyhydroxides $(\mathrm{NiOOH} \text { and } \mathrm{FeOOH})^{[18,42]}$, and amorphous cobalt-iron oxides and nickel-iron oxides $\left(\mathrm{CoFeO}_{\mathrm{x}} \text { and } \mathrm{NiFeO}_{\mathrm{x}}\right)^{[17,41]}$. For $\mathrm{H}_{2}$ evolution, platinum $(\mathrm{Pt})$ nanoparticles are still the dominant co-catalyst material ${ }^{[13,14,27]}$. There are several design characteristics that must be considered for designing and adding co-catalysts to photocatalyst/photoelectrode systems. First, the electronic interface between semiconductor and co-catalyst must be favorable for minority carrier extraction from the semiconductor. Fast extraction is needed to prevent minority carrier recombination within the semiconductor. Second, the co-catalyst must have high catalytic activity towards the desired redox reaction to be driven to prevent recombination of the charge carriers within the co-catalyst material. Lastly, the co-catalyst must have optical properties such that it does not block or compete with light absorption by the photocatalyst/photoelectrode. If the co-catalyst blocks or absorbs light that would otherwise be absorbed by the photocatalyst/photoelectrode, overall performance of the photocatalyst/PEC cell system decreases due to a smaller amount of photoexcited charge carriers being generated.

Significant problems exist with current photocatalyst/PEC cell that limit their performance below where they are economically viable to use commercially. First, photocatalyst/PEC systems are not efficient enough to be cost-effective currently. The U.S. Department of Energy placed the benchmark solar-to-hydrogen (STH) efficiency of $10 \%$ for economic viability. ${ }^{[43]}$ While some PEC systems have been demonstrated efficiencies exceeding $10 \%$, they have not proven stable for extended operation or are based on rare materials that are not economically viable for widescale production. ${ }^{[20,21]}$

One of the major causes for efficiency problems of photocatalyst/PEC cell systems is a lack of available photocatalyst/photoelectrode materials that are photostable while still able to absorb a significant part of the solar spectrum. Two examples that illustrate this dichotomy are titanium (IV) dioxide $\left(\mathrm{TiO}_{2}\right)$ and copper (I) oxide $\left(\mathrm{Cu}_{2} \mathrm{O}\right)$. Both rutile and anatase phase $\mathrm{TiO}_{2}$ are photostable with good catalytic activity towards water-oxidation. ${ }^{[4,45]}$ However, rutile and anatase $\mathrm{TiO}_{2}$ have bandgaps of $3.0 \mathrm{eV}$ and $3.2 \mathrm{eV}$ respectively - limiting light absorption to near visible and UV light and smaller wavelengths respectively that compose less than $5 \%$ of the solar spectrum. On the opposite case, $\mathrm{Cu}_{2} \mathrm{O}$ is a p-type semiconductor with a $2.1 \mathrm{eV}$ bandgap that can lead to a theoretical maximum STH efficiency of $18 \%$ based on light absorption only. ${ }^{[13,46,47]}$ However, $\mathrm{Cu}_{2} \mathrm{O}$ is not photostable due to extremely favorable kinetics for $\mathrm{Cu}^{+}$oxidation to $\mathrm{Cu}^{2+}$ using photoexcited holes. While combinations of co-catalysts have been employed to improve $\mathrm{Cu}_{2} \mathrm{O}$ stability, the added cost to the manufacturing process required to make the $\mathrm{Cu}_{2} \mathrm{O}$ stable are not economically viable currently. Without discovery of new photocatalyst/photoelectrode 
materials with narrow bandgaps that are photostable during operation, photocatalyst and photoelectrochemical cell systems will not be economically viable for production.

An additional problem with most photocatalyst/photoelectrode materials with sufficiently narrow bandgaps to absorb sunlight and photostability is poor charge transport properties. Most photocatalyst/photoelectrode materials are metal oxide semiconductors with low charge conductivity and mobility. ${ }^{[15-18]}$ With photocatalyst/photoelectrode materials with narrow bandgaps but poor charge transport, a significant amount of charge that has been photoexcited recombines before reaching the semiconductor/electrolyte interface or an external contact for extraction from the semiconductor. ${ }^{[17]}$ This premature recombination results in decreased performance of the photocatalyst/PEC cell system. While doping with various metal ions has been demonstrated to improve charge transport and performance of some photocatalyst/photoelectrode materials (i.e. $\mathrm{Mo}^{6+}$ doping of $\left.\mathrm{BiVO}_{4}\right)^{[15,16,18]}$, effective dopants to improve charge transport have not been found for all narrow bandgap photocatalyst/photoelectrode materials that are stable.

With the underlying performance considerations for semiconductor photoelectrode materials for the PEC cells discussed, the current state-of-the-art for photoelectrodes can put into context. For this, focus will be placed separately on traditional (Si or III-V) semiconductor and metal-oxide based semiconductor photoelectrodes. Peak STH efficiencies for traditional semiconductor based photoelectrodes for PEC water-splitting based on traditional semiconductors as the photoelectrode materials currently exceed the efficiencies obtained from metal-oxide based semiconductor photoelectrode materials. In 1998, John Turner's group at NREL in the United States developed a monolithic photovoltaic/photoelectrode composed of a GaAs photovoltaic cell connected to a p-type $\mathrm{GaInP}_{2}$ photoelectrode to provide the needed photovoltage to drive solar water splitting. ${ }^{[21]}$ Under 11 suns illumination, a peak STH efficiency of $12.4 \%$ was obtained. While this solar-to-hydrogen efficiency meets the U.S. DOE's target 10\% STH efficiency, the materials and manufacturing costs of the $\mathrm{GaAs}$ and $\mathrm{GaInP}_{2}$ used and stability of the overall cell are not sufficient to meet the DOE's other requirements. In 2000, Stuart Licht's group at George Washington University in the United States developed a multiple junction photoelectrolysis cell utilizing AlGaAs/Si multijunction photovoltaic cell where photoexcited electrons and holes were routed to Pt supported on carbon black and $\mathrm{RuO}_{2}$ co-catalysts respectively. ${ }^{[20]}$ This design resulted in a $18.3 \%$ STH efficiency under 1.35 suns illumination. As with the research performed by John Turner's group, the cost of $\mathrm{AlGaAs}$ and $\mathrm{RuO}_{2}$ are cost prohibitive towards widespread use despite meeting the U.S. DOE's target. Another extremely promising photoelectrode composed of traditional semiconductors for PEC water splitting developed at the Eindhoven University of Technology in the Netherlands in 2016. ${ }^{[48]}$ This photocathode is composed of $1 \mu \mathrm{m}$ long p-type $\mathrm{InP}\left(\mathrm{E}_{\mathrm{g}}=1.34 \mathrm{eV}\right)$ nanopillar arrays with overlayers of n-type doped $\mathrm{InP}(100 \mathrm{~nm})$ and n-type $\mathrm{TiO}_{2}$ to form a buried p-n junction and passivate the photoelectrode against photocorrosion, respectively. Pt nanoparticles were also decorated onto the InP nanopillars to act as a co-catalyst for $\mathrm{H}_{2}$ evolution. The resulting electric field from the buried $\mathrm{p}-\mathrm{n}$ junction extracts photoexcited electrons out of the p-type InP nanopillars, drives them towards the photoelectrode surface, and changes the band bending at the surface of the photoelectrode to increase the photovoltage from the photoelectrode. The resulting p-type $\mathrm{InP} / \mathrm{n}$-type $\mathrm{InP} / \mathrm{TiO}_{2} / \mathrm{Pt}$ nanopillars had a $15.8 \%$ applied bias photoconversion efficiency (ABPE), a metric used for measuring an equivalent STH 
efficiency for three-electrode PEC cell configurations where bias between working and reference electrodes is the measured bias instead of between working and counter electrodes, under 1 sun illumination. This value is currently the highest recorded ABPE on any single junction device. In addition, the $\mathrm{p}-\mathrm{InP} / \mathrm{n}-\mathrm{InP} / \mathrm{TiO}_{2} / \mathrm{Pt}$ photoelectrode exhibited stability during six hours of continuous operation at peak efficiency conditions. However, as with the other discussed photoelectrodes using traditional semiconductors, cost and scarcity of indium (In) needed for InP prevent the InP based photoelectrode from being commercially viable for widespread use.

The most promising metal-oxide semiconductor based photoelectrodes are typically comprised of either $\mathrm{BiVO}_{4}$, hematite $\left(\alpha-\mathrm{Fe}_{2} \mathrm{O}_{3}\right)$, or $\mathrm{Cu}_{2} \mathrm{O}$. As such, the state-of-the-art for each material will be discussed. $\mathrm{BiVO}_{4}$ is a promising n-type semiconductor with a direct bandgap of $2.4 \mathrm{eV}$. Based on semiconductor bandgap, $\mathrm{BiVO}_{4}$ has a theoretical maximum STH efficiency of 9.1\%. ${ }^{[13]}$ While this does not meet U.S. DOE STH target efficiency on its own, $\mathrm{BiVO}_{4}$ does have potential in a multi-junction photoelectrode as a wider bandgap component. $\mathrm{BiVO}_{4}$ has some advantages for solar water splitting such as its bandgap and favorable valence band energetics for driving water oxidation. However, $\mathrm{BiVO}_{4}$ suffers from poor charge transport resulting from small polaron trapping within the bulk of $\mathrm{BiVO}_{4}$ and severe surface charge recombination. ${ }^{[15,16,18]}$ $\mathrm{BiVO}_{4}$ 's charge transport can be improved by cationic doping particularly $\mathrm{Mo}^{6+}$ and/or use of 1D heterostructuring with semiconductors that have favorable band energetic alignments with $\mathrm{BiVO}_{4}$. $\mathrm{BiVO}_{4}$ 's surface charge recombination can be mitigated by rapid extraction of accumulated photoexcited charge carriers into a surface co-catalyst layer. In addition, $\mathrm{BiVO}_{4}$ is not photostable except in neutral electrolyte solutions such as phosphate buffered saline (PBS) or by coating with passivation layers that act as co-catalysts such as $\mathrm{Co}-\mathrm{Pi}$ or $\mathrm{NiFeO}_{\mathrm{x}}{ }^{[16,18]}$ The current state-of-the art for $\mathrm{BiVO}_{4}$-based photoelectrodes is $\mathrm{B} \mathrm{BiVO}_{4}$-based photoanode developed in China in 2019. ${ }^{\text {[49] }}$ This $\mathrm{BiVO}_{4}$-based photoanode is a heterostructure composed of a highly porous $\mathrm{Mo}^{6+}$ doped $\mathrm{BiVO}_{4}$ photoanode coated with thin layers of boron-doped $\mathrm{C}_{3} \mathrm{~N}_{4}$ and $\mathrm{NiFeO}_{x}$ to extract photoexcited holes out of the Mo-doped $\mathrm{BiVO}_{4}$ and act as a co-catalyst for water oxidation respectively. The $\mathrm{Mo}^{6+}$ doping content within the $\mathrm{BiVO}_{4}$ was optimized to be $0.1 \%$ mol to improve charge carrier transport within the bulk $\mathrm{BiO}_{4}$ without excess Mo to act as charge recombination centers. B-doped $\mathrm{C}_{3} \mathrm{~N}_{4}$ was chosen as a heterostructuring semiconductor due to minimal band offsets between the $0.1 \%$ Mo-doped $\mathrm{BiVO}_{4}$ and $\mathrm{B}$-doped $\mathrm{C}_{3} \mathrm{~N}_{4}$ that enabled for effective extraction of photoexcited holes with minimal overpotential losses. The use of $\mathrm{NiFeO}_{\mathrm{x}}$ as a co-catalyst limits recombination of the photoexcited holes within the outer B-doped $\mathrm{C}_{3} \mathrm{~N}_{4}$ layers while being mostly transparent to incident light. The $0.1 \%$ Mo-doped $\mathrm{BiVO}_{4} / \mathrm{B}$-doped $\mathrm{C}_{3} \mathrm{~N}_{4} / \mathrm{NiFeO}_{\mathrm{x}}$ photoanode has shown a $2.67 \%$ ABPE in a neutral electrolyte with an $8 \%$ loss in photocurrent after 10 hours of operation at $1.23 \mathrm{~V}$ vs. RHE. The $2.67 \%$ ABPE is significantly below the target $10 \% \mathrm{STH}$ efficiency. In addition, this $0.1 \%$ Mo-doped $\mathrm{BiVO}_{4} / \mathrm{B}$-doped $\mathrm{C}_{3} \mathrm{~N}_{4} / \mathrm{NiFeO}_{x}$ photoanode has not been tested in a two-electrode PEC cell configuration consistent with a realistic commercial system. The main source for the relatively low ABPE versus the STH efficiency limit for $\mathrm{BiVO}_{4}-$ based photoanodes is the slow rise in photocurrent at low potentials despite the use of heterostructures and co-catalysts. As such, there is significant room for performance improvement with $\mathrm{BiVO}_{4}$ photoelectrodes. 
$\alpha-\mathrm{Fe}_{2} \mathrm{O}_{3}$ is another promising n-type semiconductor with an indirect bandgap of $2.0 \mathrm{eV}$ and direct bandgap of $2.2 \mathrm{eV}$. Based on these bandgaps, $\alpha-\mathrm{Fe}_{2} \mathrm{O}_{3}$ has a maximum theoretical STH efficiency of $12.9 \%$, high enough to meet the U.S. DOE efficiency target. ${ }^{[13]}$ The main advantages of $\alpha-\mathrm{Fe}_{2} \mathrm{O}_{3}$ as a photoelectrode are its narrow bandgap, photostability, sufficient valence band energetics to drive water oxidation, and its composition being made from one of the most abundant metals available in iron. However, $\alpha-\mathrm{Fe}_{2} \mathrm{O}_{3}$ also has several problems that have limited its potential for PEC water splitting. $\alpha-\mathrm{Fe}_{2} \mathrm{O}_{3}$ has extremely poor transport due to small polaron trapping. ${ }^{50,51]}$ While $\mathrm{BiVO}_{4}$ has been able to overcome small polaron trapping through doping with $\mathrm{Mo}^{6+}$, small polaron trapping within $\alpha-\mathrm{Fe}_{2} \mathrm{O}_{3}$ has not been overcome despite widespread testing of various cationic dopants for $\alpha-\mathrm{Fe}_{2} \mathrm{O}_{3}$. In addition, surface trap states that form in response to adsorbed intermediate products during solar water-splitting trap photoexcited charge carriers and result in Fermi level pinning. ${ }^{[50]}$ The consequences of the surface trap state formation and Fermi level pinning are increased surface charge carrier recombination and a significantly lower generated photovoltage versus what should be expected based on $\alpha-\mathrm{Fe}_{2} \mathrm{O}_{3}$ 's bandgap. The lower generated photovoltage when coupled with poor catalytic activity of $\alpha-\mathrm{Fe}_{2} \mathrm{O}_{3}$ towards water-splitting results in a high onset potential for photocurrent that compromises $\alpha-\mathrm{Fe}_{2} \mathrm{O}_{3}$ 's STH efficiency. Despite these problems, significant research focus has been spent on overcoming the charge transport issues, low photovoltage, and catalytic activity. The current state-of-the-art for $\alpha-\mathrm{Fe}_{2} \mathrm{O}_{3}$ photoelectrodes is the $\alpha-\mathrm{Fe}_{2} \mathrm{O}_{3}$ photoanode developed at POSTECH in South Korea in 2017. ${ }^{[52]}$ With this $\alpha-\mathrm{Fe}_{2} \mathrm{O}_{3}$ photoanode, 1-D $\alpha-\mathrm{Fe}_{2} \mathrm{O}_{3}$ nanorod arrays $(\sim 730 \mathrm{~nm}$ long and $20-30 \mathrm{~nm}$ in diameter) were grown on FTO glass by hydrothermal methods and then subsequently heated under $\mathrm{H}_{2}$ at $350{ }^{\circ} \mathrm{C}$ to induce oxygen vacancies and $\mathrm{Fe}^{2+}$ formation within the $\alpha-\mathrm{Fe}_{2} \mathrm{O}_{3}$ nanorods. The induced $\mathrm{Fe}^{2+}$ states within the $\alpha-\mathrm{Fe}_{2} \mathrm{O}_{3}$ nanorods act as additional small polaron hopping sites for improved charge transport. In addition, a $3.5 \mathrm{~nm}$ thick amorphous $\mathrm{TiO}_{2}$ layer and Co-Pi layer to act as passivation and co-catalyst layers respectively. The amorphous $\mathrm{TiO}_{2}$ layer was also treated under $\mathrm{H}_{2}$ at $200-250{ }^{\circ} \mathrm{C}$ to boost the conductivity of the layer to further allow for photoexcited holes from the $\alpha-\mathrm{Fe}_{2} \mathrm{O}_{3}$ nanorods to reach the photoanode surface. The $\alpha-\mathrm{Fe}_{2} \mathrm{O}_{3} / \mathrm{TiO}_{2} / \mathrm{Co}-\mathrm{Pi}$ photoanode was ultimately able to generate photocurrents of $6 \mathrm{~mA} / \mathrm{cm}^{2}$ under 1 sun illumination at an applied bias of $1.23 \mathrm{~V}$ vs. RHE (the thermodynamic potential for solar water-splitting). This photocurrent is comparable to the state-of-the-art $\mathrm{BiVO}_{4}$ photoelectrode previously discussed. While the ABPE was not calculated in this paper, the relatively high photocurrent onset potential ( $\sim 0.65 \mathrm{~V}$ vs. RHE) and potential where photocurrent saturation begins ( $\sim 0.8 \mathrm{~V}$ vs. RHE) for this photoanode results in a lower $\mathrm{ABPE}$ in comparison to the state-of-the-art $\mathrm{BiVO}_{4}$ photoelectrode. The $\alpha-\mathrm{Fe}_{2} \mathrm{O}_{3} / \mathrm{TiO}_{2} / \mathrm{Co}-\mathrm{Pi}$ photoanode does exhibit photostability with a negligible decrease in performance after 100 hours of continuous operation. The main bottleneck for improving the performance of the $\alpha-\mathrm{Fe}_{2} \mathrm{O}_{3} / \mathrm{TiO}_{2} / \mathrm{Co}-\mathrm{Pi}$ photoanode still remains charge transport and separation within the $\alpha-\mathrm{Fe}_{2} \mathrm{O}_{3}$ nanorods even after the $\mathrm{H}_{2}$ treatment as $\eta_{\text {sep }}$ does not exceed $50 \%$.

$\mathrm{Cu}_{2} \mathrm{O}$ is a promising p-type semiconductor with a direct bandgap of $2.1 \mathrm{eV}$. As stated previously, based on bandgap, $\mathrm{Cu}_{2} \mathrm{O}$ has a theoretical STH efficiency of $18 \%$, far beyond the U.S. DOE efficiency target efficiency. $\mathrm{Cu}_{2} \mathrm{O}$ has additional advantages for solar water-splitting due to it being composed of earth abundant $\mathrm{Cu}$ and sufficient conduction band energy to drive the hydrogen evolution reaction. ${ }^{[46,47]}$ However, $\mathrm{Cu}_{2} \mathrm{O}$ has several disadvantages for solar watersplitting as well. As previously mentioned, $\mathrm{Cu}_{2} \mathrm{O}$ has poor photostability in aqueous electrolytes. 
The poor photostability has been the most difficult problem to overcome historically for $\mathrm{Cu}_{2} \mathrm{O}$. However, recent breakthroughs with protective overlayer materials and manufacturing have significantly improved the photostability of $\mathrm{Cu}_{2} \mathrm{O}$-based photocathodes. ${ }^{[46,}{ }^{47]} \mathrm{Cu}_{2} \mathrm{O}$ has low absorption coefficients at wavelengths above $500 \mathrm{~nm}$ despite its bandgap and poor electron transport properties. ${ }^{[53]}$ The low absorption coefficients and poor electron transport can be overcome using nanostructuring the $\mathrm{Cu} 2 \mathrm{O}$ such as creating 1-D nanorod arrays to trap light and limit required electron diffusion lengths within the $\mathrm{Cu}_{2} \mathrm{O}$ photocathodes. In addition, doping $\mathrm{Cu}_{2} \mathrm{O}$ with $\mathrm{Na}^{+}$can significantly improve electron transport within $\mathrm{Cu}_{2} \mathrm{O}{ }^{[54]} \mathrm{Cu}_{2} \mathrm{O}$ also has poor catalytic activity towards the hydrogen evolution reaction which can be overcome with the use of cocatalysts. The current state-of-the-art for $\mathrm{Cu}_{2} \mathrm{O}$-based photocathodes was created by Michael Grätzel's research group in 2018. ${ }^{[55]}$ With this $\mathrm{Cu}_{2} \mathrm{O}$-based photocathode, a $\mathrm{Cu}_{2} \mathrm{O}$ nanorod array (3-5 $\mu \mathrm{m}$ long, 200-400 $\mathrm{nm}$ in diameter) is grown on a copper coated FTO glass substrate. Thin $\mathrm{n}-$ type $\mathrm{Ga}_{2} \mathrm{O}_{3}$ and amorphous $\mathrm{TiO}_{2}$ overlayers are grown on the $\mathrm{Cu}_{2} \mathrm{O}$ nanorod array to aid in extraction of photoexcited photoelectrons from the $\mathrm{Cu}_{2} \mathrm{O}$ and passivate the $\mathrm{Cu}_{2} \mathrm{O}$ nanorods against photocorrosion respectively. The use of $\mathrm{Ga}_{2} \mathrm{O}_{3}$ as the n-type charge extraction material was chosen due to its minimal conduction band energy difference with $\mathrm{Cu}_{2} \mathrm{O}$, maximizing the photovoltage generated within the photocathode. The $\mathrm{Cu}_{2} \mathrm{O} / \mathrm{Ga}_{2} \mathrm{O}_{3} / \mathrm{TiO}_{2}$ nanorod arrays were also coated with either a $\mathrm{RuO}_{\mathrm{x}}$ or a NiMo co-catalyst layer to improve the catalytic activity of the photocathode for hydrogen evolution. The resulting $\mathrm{Cu}_{2} \mathrm{O} / \mathrm{Ga}_{2} \mathrm{O}_{3} / \mathrm{TiO}_{2} / \mathrm{RuO}_{x}$ photocathode showed photocurrent onset for hydrogen evolution at $+1.0 \mathrm{~V}$ vs. RHE with photocurrents of $10 \mathrm{~mA} / \mathrm{cm}^{2}$ at $0 \mathrm{~V}$ vs. RHE (the thermodynamic potential for hydrogen evolution) under 1 sun illumination in an aqueous electrolyte at $\mathrm{pH}$ 5. In addition, the $\mathrm{Cu}_{2} \mathrm{O} / \mathrm{Ga}_{2} \mathrm{O}_{3} / \mathrm{TiO}_{2} / \mathrm{RuO}_{\mathrm{x}}$ photocathode showed stability for over 100 hours of continuous testing. However, $\mathrm{Ru}$ is too scarce to be used on wide scale. After switching the RuOx for the NiMo composed of earth-abundant metals, the resulting $\mathrm{Cu}_{2} \mathrm{O} / \mathrm{Ga}_{2} \mathrm{O}_{3} / \mathrm{TiO}_{2} / \mathrm{NiMo}$ nanorod array photocathode shows slightly lower photocurrent ( 7.5 $\mathrm{mA} / \mathrm{cm}^{2}$ at $0 \mathrm{~V}$ vs. RHE under 1 sun illumination in an aqueous electrolyte at $\mathrm{pH}$ 9) due to the lower catalytic activity of the NiMo co-catalyst versus the RuOx co-catalyst. Stability testing showed only a $10 \%$ decrease in photocurrent after 8 hours. Further, a planar (i.e. not a nanorod array) version of the $\mathrm{Cu}_{2} \mathrm{O} / \mathrm{Ga}_{2} \mathrm{O}_{3} / \mathrm{TiO}_{2} / \mathrm{NiMo}$ photocathode was placed in tandem with a n-type $\mathrm{BiVO}_{4}$ photoanode tested for performance. The tandem $\mathrm{Cu}_{2} \mathrm{O} / \mathrm{BiVO}_{4} \mathrm{PEC}$ system had a STH in excess of $3 \%$ and significantly improved versus other tandem photoelectrodes that utilized traditional semiconductor photoelectrodes as one of the photoelectrodes in the PEC cell. As such, $\mathrm{Cu}_{2} \mathrm{O}$-based photocathodes are currently the closest metal-oxide photoelectrode materials capable of meeting efficiency and cost requirements.

\subsection{Metal-Organic Frameworks}

Metal-organic frameworks (MOFs) are a class of materials built around nanometer scale metal-based inorganic nodes interconnected by organic linker ligands. ${ }^{[56,57]}$ Metal-organic frameworks have significantly tunable chemical, electronic, and optical properties due to the large variety of combinations of metal-based nodes and organic linkers available. MOFs are highly 
crystalline due to ordered nature of the inorganic-organic network. This crystallinity tends to give MOFs high thermal and chemical stability depending on the choice of constituent nodes and linkers in comparison to purely organic compounds. In addition, MOFs are highly porous due to the frame-like structure formed by the node-ligand networks. Due to the combination of porosity and tunable properties, MOFs are researched for use as batteries, electrocatalysts, photocatalysts, gas storage, etc. This section will primarily be focused on MOFs as candidates for electrocatalyst and photocatalyst materials. ${ }^{[56-58]}$

Photocatalysts based on MOFs can be classified based on the nature of their photoabsorber component and catalytic centers. Three different cases summarize the possible classes of MOF photocatalysts. With the first case, the photoabsorber material are inorganic semiconductor quantum dots forming the nodes of the MOF (Figure 2.6). ${ }^{[57-60]}$ The organic linker network serves to separate the quantum dots from each other to create a porous network of isolated photocatalysts in the MOF. The porous nature of the MOF allows for mass transfer of reaction products and reactants away from the quantum dots provided that the pores of the MOF are sufficiently large enough for transport.

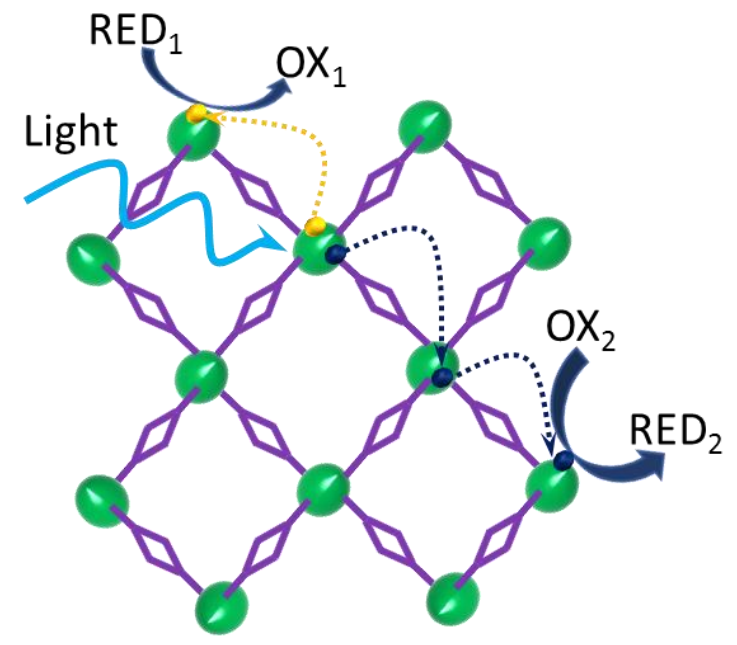

Figure 2.6: MOF-based photocatalyst with photoactive semiconductor quantum dot nodes as photoexcited charge generation sites.

The second case is the organic linker ligands that connect the nodes in the MOF are organic semiconductor materials (i.e. dye molecules) that serve as the photoabsorber material (Figure 2.7). ${ }^{[31,57,61]}$ The metal-based nodes can serve as connectors between organic semiconductor ligands and as potential catalyst sites for photocatalytic reactions. Due to the nature of the linker ligands as organic semiconductors, charge separation and transport, excitons (bound electron-hole pairs) rather than free electrons and holes are formed. As such, MOFs utilizing organic semiconductor ligands must be designed to facilitate easy transport of excitons and its subsequent separation into free electrons and holes. 


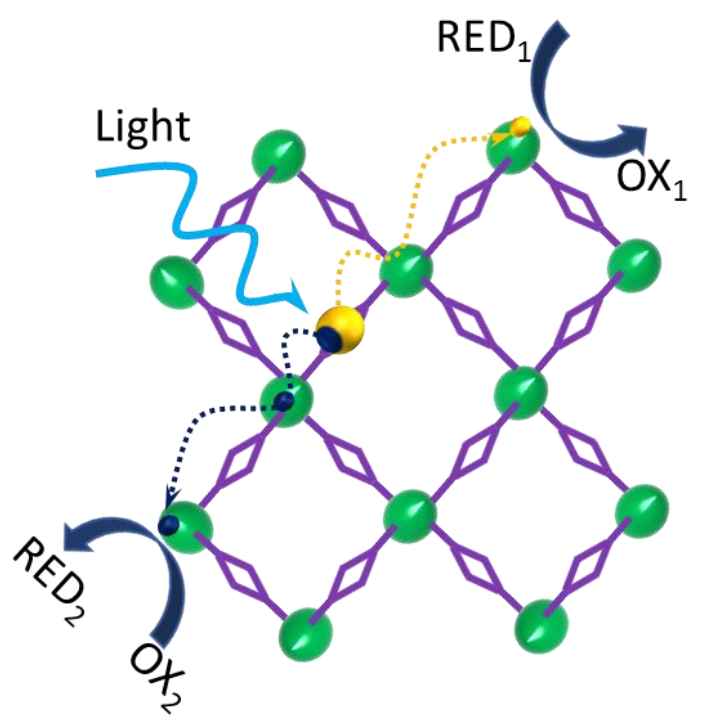

Figure 2.7: MOF-based photocatalyst with organic semiconductor linkers as photoexcited exciton generation sites with subsequent charge carrier separation, transport and catalysis at MOF's metalbased nodes.

The last case is that a separate photoabsorber (inorganic or organic) is introduced into the porous structure of the MOF (Figure 2.8). In this case, the MOF serves as a high surface area scaffold to serve a complementary role to the introduced photoabsorber such as acting as a catalyst or conductive material for charge extraction purposes. In this case, the MOF's pore structure must be sufficiently large to allow for photoabsorber uptake. In addition, the MOF must facilitate charge extraction from the introduce photoabsorber. ${ }^{[57]}$ 


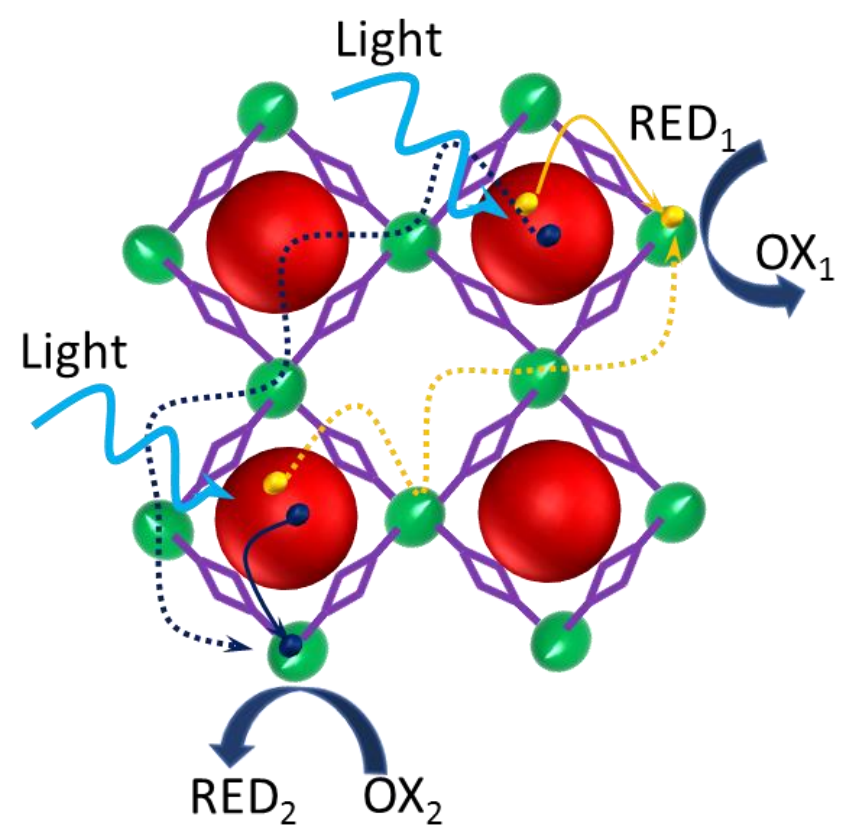

Figure 2.8: MOF-based photocatalyst with photoactive semiconductor quantum dots introduced into the MOF's pore network acting as photoexcited charge generation sites with charge carrier hopping and catalysis at MOF's metal-based nodes.

MOF synthesis is done primarily by two different methods. In the first method, MOF particles can be synthesized through solvothermal methods where node and linker precursors are dissolved in a solvent and heated to elevated temperatures. ${ }^{[59-61]}$ The synthesized MOF particles are typically hundreds of nanometers in size. While MOF particles of that size are suitable for particle-based photocatalysts, they are difficult to form into dense films needed to form photoelectrodes for use as in a PEC cell. The second method for MOF synthesis is a layer-by-layer deposition approach where ligands and metal-based nodes are deposited in repeatedly alternating steps. ${ }^{[62]}$ This approach can yield conformal MOF thin films on a variety of substrates - including nanostructured substrates. However, the need for repeated, time-consuming deposition steps for layer-by-layer MOF deposition steps makes thick films greater than 50-100 nm impractical to synthesize. While physically thin films have advantages for charge transport and charge collection, physically thin films are very rarely optically thick. To compensate for the lack of optical thickness with thin MOF films, light trapping techniques may be employed.

There are candidate MOFs (UiO-66, ZIF-8, NTU-9, MIL-125, and the PCN-22x family) that have shown the most promise for photocatalytic/PEC applications for driving various chemical reactions. Of these MOFs, UiO-66 is the most explored MOFs for photocatalysis. UiO66 is a MOF with Zr-based nodes with 1,4-benzodicarboxylic acid linker ligands. ${ }^{[63-68]} \mathrm{UiO}-66$ is mostly chemically stable. Pristine UiO-66 has a wide bandgap of $3.5 \mathrm{eV}$, thus limiting its light absorption to the UV spectrum. However, a few modifications to the UiO-66 MOF can extend its absorption to the visible spectrum. Functionalizing the 1,4-benzodicarboxylic acid linker ligands 
with groups such as $-\mathrm{NO}_{2}$ and $-\mathrm{NH}_{2}$ results in new electronic states within the bandgap that enables visible light absorption up to $450 \mathrm{~nm} .{ }^{[63,64]}$ Additionally, partial substitution of the 1,4 benzodicarboxylic acid linker ligands with other ligands such as 2-aminoterepthalic acid can also introduce additional electronic states within UiO-66's bandgap to further enable visible light absorption. ${ }^{[63]}$ Lastly, partial substitution of the $\mathrm{Zr}$ from within the UiO-66 nodes with other elements such as Ti can also introduce new electronic states to redshift the absorption of the UiO66. ${ }^{[65]}$ It is important to note that these general approaches to modifying UiO-66 to redshift its absorption to visible wavelengths are applicable to other MOFs as well. UiO-66 has been used as a photocatalyst for dye degradation ${ }^{[65,67]}$, reduction of heavy metal ions such as $\mathrm{Cr}^{6+[67]}$, hydrogen evolution ${ }^{[68]}, \mathrm{CO}_{2}$ reduction to $\mathrm{HCOO}-{ }^{[63]}$, and organic transformation reactions such as conversion of benzyl alcohol to benzaldehyde. ${ }^{[66]}$ The relative chemical stability, tunability of the electronic structure, and large variety of catalytic reactions that can be driven highlight the potential of UiO66 as a photocatalyst material.

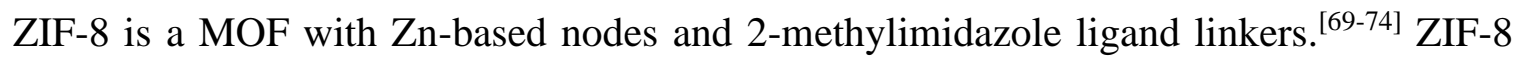
has a wide bandgap of $5.0 \mathrm{eV}$, limiting its light absorption and photocatalytic activity to the UV spectrum only. ZIF-8 has shown some photocatalytic activity towards dye degradation and reduction of $\mathrm{Cr}(\mathrm{VI}) .{ }^{[71-74]}$ However, part of ZIF-8's main appeal as a MOF photocatalyst is its similarity to zeolite materials that are commonly used for adsorption and capture of gases such as $\mathrm{CO}_{2}$. As such, ZIF-8 is commonly used in heterostructures as an overlayer with other semiconductors such as $\mathrm{ZnO}^{[69,70]}, \mathrm{Zn}_{2} \mathrm{GeO}_{4}{ }^{[71]}$, and $\mathrm{TiO}_{2}{ }^{[73,74]}$ as a gas capture and cocatalyst material whose photocatalytic activity supplements the photocatalytic activity of the underlying semiconductor in the heterostructure.

NTU-9 is a MOF with Ti-based nodes and 2,5-dihydroxyterephthalic acid ligand linkers. ${ }^{[60,}$ 75] The main advantages of NTU-9 as a photocatalyst or photoelectrode material for PEC cells are its p-type semiconducting behavior, narrow bandgap $(1.74 \mathrm{eV})$, and conduction band sufficiently negative enough to drive the solar water-splitting reaction among other reactions. In addition, NTU-9 has shown activity towards driving photocatalytic reactions under visible light by itself and when used in heterostructures with CdTe nanorods. ${ }^{[60,75]}$ However, as with several other MOF materials, low carrier concentrations $\left(\sim 10^{15} \mathrm{~cm}^{-3}\right)$ and poor charge separation and transport currently limit the performance of this MOF for photocatalytic applications. ${ }^{[60]}$

MIL-125 is a MOF with Ti-based nodes and 1,4-benzodicarboxylic acid ligand linkers. ${ }^{[59,}$ ${ }^{76-78]}$ Pristine MIL-125 has a wide bandgap of $3.6 \mathrm{eV}$ that limits its photocatalytic activity to the UV spectrum only. However, like in the case of UIO-66, $-\mathrm{NH}_{2}$ functionalization of the 1,4benzodicarboxylic acid linkers introduces new electronic states within the bandgap that extends light absorption to the visible spectrum up to wavelengths of $550 \mathrm{~nm}$. ${ }^{[59,76-78]}$ The $-\mathrm{NH}_{2}$

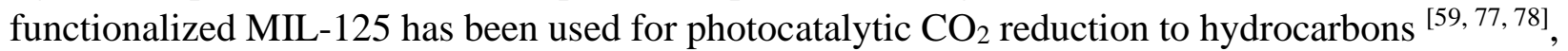
dye degradation ${ }^{[78]}$, and solar water-splitting. ${ }^{[76]}$ As with most MOFs, poor charge transport and separation limit the photocatalytic performance of (pristine and $-\mathrm{NH}_{2}$ functionalized) MIL-125 MOF. However, some research on utilizing heterostructures composed of thin $-\mathrm{NH}_{2}$ functionalized MIL-125 layers on 1-D $\mathrm{TiO}_{2}$ nanorod arrays show the promise of $-\mathrm{NH}_{2}$ functionalized MIL-125 $\mathrm{MOF}$ as a photocatalyst material with visible light activity. ${ }^{[76]}$ 
PCN-22x ( $\mathrm{x}=2-5)$ is a family of MOFs with Zr-based nodes and tetrakis(4carboxyphenyl) porphyrin ligand linkers. ${ }^{[79-83]}$. One of the major disadvantages of porphyrinic MOFs is the degradation of the MOFs due to photobleaching of the porphyrin linkers. However, the Zr-based nodes, similar to those used in UiO-66, provide some chemical stability to the PCN$22 x$ family while the porphyrinic linkers are strong light absorption within the visible spectrum up to wavelengths of $750 \mathrm{~nm}$. In addition, the structure of porphyrin molecules enables incorporation of different transition metal cations such as $\mathrm{Fe}^{3+}$ that can be used to enhance the catalytic activity of PCN-22x MOFs. ${ }^{[79,81,82]}$ The PCN-22x family of MOFs have been demonstrated as visible light active photocatalysts for $\mathrm{CO}_{2}$ reduction to HCOO- ${ }^{[80]}$ and ordinary catalysts for organic transformation reactions such as transformation of benzaldehydes. ${ }^{[79,81,82]}$ Research presented within this dissertation focuses on using PCN-225 in heterostructures for solar water-splitting. However, the charge carriers generated within the porphyrin linker ligands are excitonic in nature resulting in extremely poor charge separation within PCN 22x MOFs. Nonetheless, the wide spectrum of light absorption by the PCN-22x MOF is appealing for future research.

\subsection{Plasmonics for Light Energy Harvesting Applications}

Plasmonics is a field of research focused on utilizing surface plasmon resonance (SPR), a phenomenon where light illumination photoexcites the surface electrons of certain metals and degenerate semiconductors to coherently oscillate, for various applications. For the purposes of this dissertation, focus will be on plasmonic metals only despite recent growth in research on plasmonic semiconductors. Typical plasmonic metals of interest include gold (Au), silver (Ag), copper $(\mathrm{Cu})$, aluminum $(\mathrm{Al})$ since their plasmonic resonance occur within the UV-Visible-NIR spectrum that can be utilized for energy and technological applications such as telecommunications. SPR can occur in two forms - surface plasmon polaritons (SPP) (Figure 2.9a) and localized surface plasmon resonance (LSPR) (Figure 2.9b). Both forms of SPR can have applications in light and solar energy harvesting. 


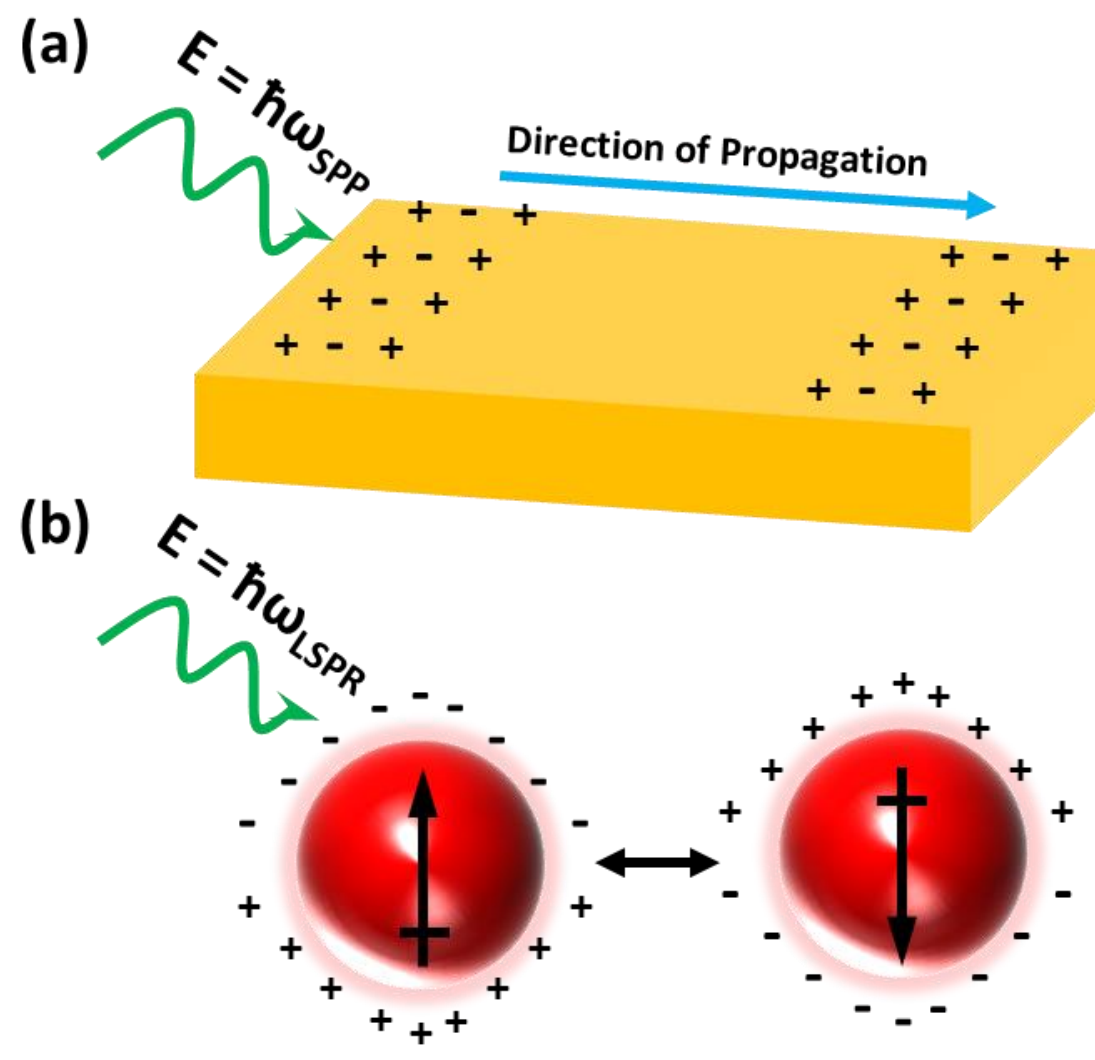

Figure 2.9: Types of surface plasmon resonance (SPR); (a) surface plasmon polaritons; (b) localized surface plasmon resonance (LSPR).

In the case of surface plasmon polaritons (SPP), the excited electron oscillations propagate along the interface of the plasmonic metal and the surrounding dielectric medium. ${ }^{[84,85]}$ This propagating wave of oscillating electrons can be utilized for light-trapping within semiconductors for solar energy harvesting applications due to the considerable generated electromagnetic fields along the plasmonic metal / dielectric interface. However, excitation of SPPs requires an additional source of momentum or employing grating to make up for a difference in dispersion between a photon and SPP. The need for additional momentum is a design consideration before utilizing SPP for optoelectronic devices. ${ }^{[85]}$

In the case of localized surface plasmon resonance (LSPR), the plasmonic material has a characteristic dimension much smaller than the wavelength of light (i.e. nanoparticles (NPs)) used to excite the LSPR. ${ }^{[84-86]}$ Due to the confinement of the plasmon to the surface of the plasmonic $\mathrm{NP}$, the dispersion relation of the LSPR does not have a momentum mismatch with the light used to excite it. ${ }^{[85]}$ As a result, plasmonic metal NPs have extremely large absorption and/or scattering cross-sections relative to their physical size. This gives plasmonic metal nanoparticles and nanostructures great potential as photosensitizers for solar energy. In addition, the confined but coherent oscillation of electrons with LSPR results in extremely high localized electromagnetic 
(EM) fields around the plasmonic metal nanoparticles. This is useful in both solar energy harvesting ${ }^{[85-96]}$ and for sensors based on surface enhanced Raman scattering (SERS). ${ }^{[84]}$ The research within this dissertation uses LSPR and not SPP as the SPR type of interest. As such, all further discussion will be focused on LSPR from plasmonic NPs and its applications towards enhancing the PEC performance of semiconductor photoelectrodes. In addition, additional effects of plasmonic NPs that are non-plasmonic in nature that can enhance the PEC performance of semiconductor photoelectrodes will be discussed as to highlight how to distinguish between plasmonic and non-plasmonic effects of plasmonic metal NPs.

\subsubsection{Plasmonic Effects for Modulating Semiconductor Photoconversion}

The LSPR from plasmonic nanoparticles has many appeals for use in light harvesting and sensing applications. One of the most appealing traits of plasmonic nanoparticles and nanostructures is the high tunability of their optical properties by varying different properties such as shape, size, and material of the plasmonic nanoparticles and nanostructures. To illustrate the effect of changing plasmonic material, $15 \mathrm{~nm}$ diameter silver (Ag) nanospheres have LSPR at wavelengths of $\sim 400 \mathrm{~nm}$ while gold $\mathrm{(Au}$ ) nanospheres of comparable size have a LSPR at wavelengths of $\sim 520 \mathrm{~nm}$. ${ }^{[97]}$ To illustrate the effects of size on the LSPR of plasmonic nanoparticles, the LSPR of $15 \mathrm{~nm}$ diameter Au nanospheres is $\sim 520 \mathrm{~nm}$ while the LSPR of $50 \mathrm{~nm}$ diameter Au nanospheres red-shifts to $\sim 535 \mathrm{~nm} .{ }^{[98]}$ Furthermore, increasing the size of plasmonic nanoparticles also increases the relative contribution of scattering versus absorption towards the overall extinction spectrum of the plasmonic nanoparticle. To illustrate the effects of shape, 60 $\mathrm{nm} \mathrm{Au}$ nanospheres have LSPR at wavelengths around $550 \mathrm{~nm}$. By changing the shape from nanospheres to nanostars with multiple sharp tips, the LSPR can be red-shifted into the NIR part of the spectrum with LSPR between 750 - $800 \mathrm{~nm}$ for Au nanostars of similar size. ${ }^{[99]}$ In addition, sharp corners and edges serve as focal points for LSPR - resulting in further amplified local EM fields around the sharp features of the Au nanostars and similar plasmonic nanoparticles and nanostructures with sharp features.

To effectively utilize LSPR for light energy harvesting, an understanding of how the LSPR and constituent electrons behave from excitation to complete relaxation. First, incident light is absorbed and the LSPR is excited. The electrons that compose the LSPR continue to oscillate coherently for between $20 \mathrm{fs}$ and $30 \mathrm{fs}$ after initial LSPR excitation. After the first 20-30 fs, the electron oscillations begin to lose coherence due to Landau damping. ${ }^{[86,87,90,67]}$ After 100 fs from initial excitation, the LSPR ceases completely. The LSPR's energy may be relaxed either through radiative scattering (typical of larger plasmonic nanoparticles) or released non-radiatively (typical of smaller plasmonic nanoparticles). In the case of non-radiative relaxation, there still exists a population of "hot" electrons and "hot" holes with energies above the Fermi level and below the Fermi level of the metal nanoparticle with a distribution that can be described as thermal distribution at an elevated temperature relative to the bulk plasmonic nanoparticle immediately after the LSPR has fully decayed. These hot electrons and holes will relax back to the Fermi level of the metal over the course of $10 \mathrm{~ns}$ due to electron-phonon scattering (100 fs -1 ps after LSPR 
excitation) and electron-electron scattering ( $1 \mathrm{ps}-10 \mathrm{~ns}$ after LSPR excitation). There are multiple energy transfer processes that can occur at different timescales during the LSPR's lifetime when the plasmonic metal is placed in contact or near a semiconductor.

The first LSPR energy transfer process that is possible is plasmon-induced resonant energy transfer (PIRET) (Figure 2.10). ${ }^{[86,}$ 90, 91, 93-96] plasmonic NPs with diameters or characteristic dimensions with less than $50 \mathrm{~nm}$ can absorb light and then directly transfer the energy from the plasmonic NPs' LSPR into nearby semiconductor acceptors. PIRET is a non-radiative resonant energy transfer process, discovered by Nianqiang Wu's research group in 2012, where energy stored in the LSPR of a plasmonic NPs such as Au NPs is transferred via dipole-dipole interactions similar to Förster resonant energy transfer (FRET) to form new excited electron-hole pairs within a nearby semiconductor acceptor. ${ }^{[89-91,93,100]}$ This process occurs while the plasmon is still coherent $(\mathrm{t} \leq 30 \mathrm{fs}$ after plasmon excitation). As with scattering by Au NPs, there are pre-requisites for PIRET to occur between Au NPs and semiconductors. For PIRET to occur, there must be a spectral overlap between the Au NPs' LSPR and the absorption spectrum of the semiconductor acceptor with the Au NPs' LSPR red-shifted with respect to the semiconductor acceptor's absorption edge. PIRET's energy transfer will follow the spectral overlap integral between the plasmonic Au NP donor and semiconductor acceptor. As in the case of FRET, PIRET has a 1/r distance dependence on the energy transfer efficiency. Therefore, the Au NPs and semiconductor must be near to (i.e. within $10 \mathrm{~nm}$ with less separation being better) but not necessarily in contact with each other to enable PIRET. In addition, interface effects between the Au NPs and the semiconductor must be accounted for as well since electron transfer between the Au NPs and semiconductor can result in early dephasing of the Au NPs LSPR and decreased PIRET efficiency. Since PIRET is a coherent resonant energy transfer process, the dephasing times of both the $\mathrm{Au}$ NPs' LSPR and the semiconductor are significant factors on the efficiency of PIRET. In general, it is preferable to have a smaller dephasing time with the semiconductor versus the Au NPs to prevent back-transfer of energy to the Au NPs from the semiconductor via FRET. While dephasing times for Au NPs or plasmonic nanoparticles can be determined from the LSPR spectrum lineshape, semiconductor dephasing times are difficult to measure and not readily available in literature. ${ }^{[101]}$ This lack of known dephasing times for semiconductors hinders designing devices that utilize PIRET from Au NPs to semiconductors. PIRET is still a promising method for transferring the energy stored in plasmons into nearby acceptors with over $30 \%$ of the plasmon's energy being transferred into the semiconductor acceptor. ${ }^{[90,95]}$ 


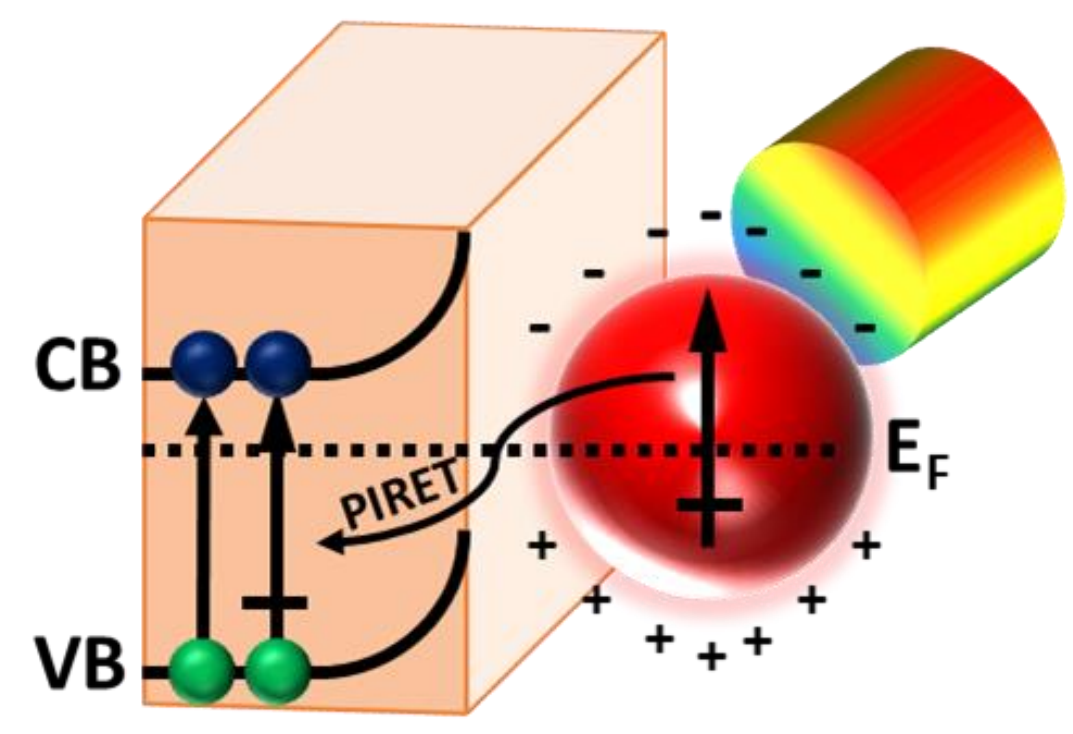

Figure 2.10: Schematic for plasmon-induced resonant energy transfer between plasmonic nanoparticles and a n-type semiconductor.

The second LSPR energy transfer process that is possible is radiative scattering of the LSPR's energy as light (Figure 2.11). ${ }^{[8,86]}$ Scattering occurs as the immediately after the plasmon decays ( $\sim 100$ fs after light excitation Since the plasmon's energy released during scattering takes the form of light photons, the semiconductor coupled does not need to be in direct contact with plasmonic NPs. In addition, large scattering cross-sections are typical of plasmonic nanostructures only if they are above a certain size. For example, scattering for plasmonic nanospheres only begin to dominate absorption when the nanosphere is greater than $50 \mathrm{~nm}$ in dimension. ${ }^{[85]}$ Depending on the size and distribution of the plasmonic NPs with respect to the coupled semiconductor, light may be scattered multiple times within the semiconductor before total absorption, resulting in light-trapping within semiconductor. ${ }^{[85,102,103]}$ The resulting increased effective optical pathlengths from scattering and light trapping are useful especially when dealing with semiconductor thin films with low absorption coefficients. However, since the coupled semiconductor within the heterostructure must absorb wavelengths matching the scattered light from the plasmonic NPs for the light to be harvested and converted into useful current, scattering from plasmonic NPs cannot extend the spectrum of useful wavelengths for a light harvesting device. 


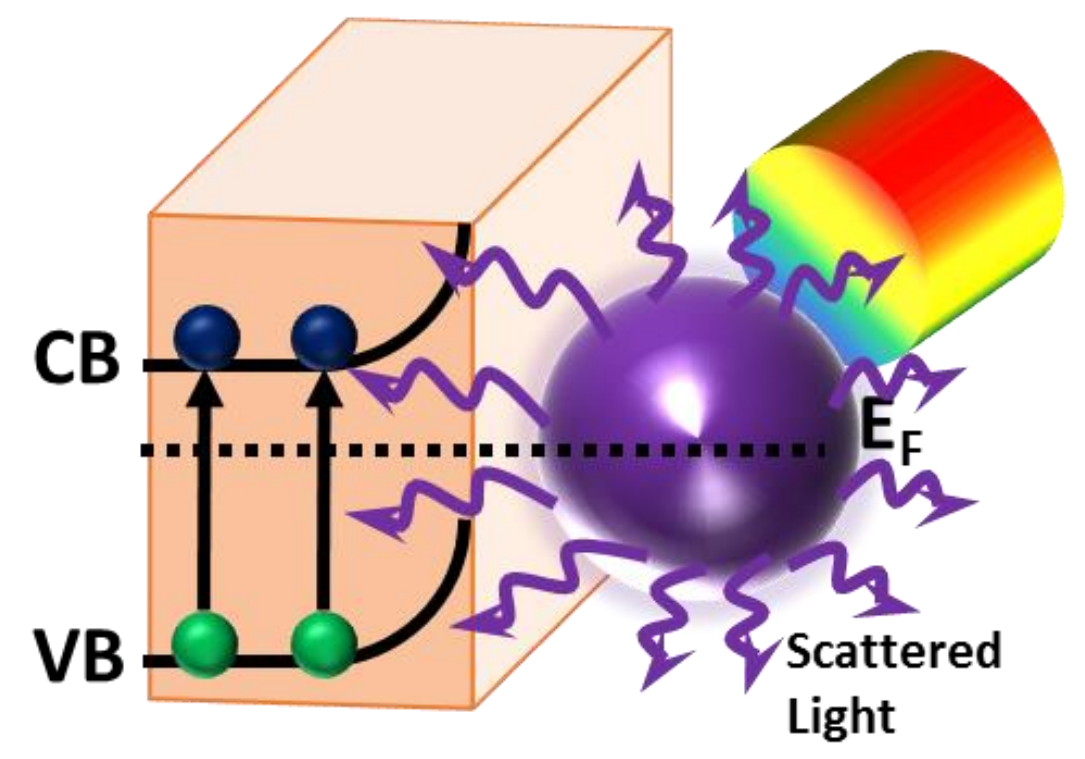

Figure 2.11: Schematic for radiative scattering of light by plasmonic nanoparticles resulting in increased light absorption and charge carrier generation within a n-type semiconductor.

The third LSPR energy transfer process that can occur is injection of energetic hot carriers from plasmonic metal to semiconductor (Figure 2.12). ${ }^{[94-96,99-100,102,104-106]}$ Hot carrier injection (also known as plasmon-induced charge separation (PICS)) was discovered by Dr. Tetsu Tatsuma's group studying Au NPs on $\mathrm{TiO}_{2}$ in 2004. ${ }^{[104-109]}$ The energetic hot charge carriers are a remnant of the plasmonic NPs LSPR after the LSPR has lost coherence and have energy distributions that follow the plasmonic NPs' LSPR. Like with radiative scattering, hot carrier injection starts to occur after the LSPR loses coherence since hot carriers are only generated in the plasmonic metal after Landau damping has occurred. As such, subsequent hot carrier transfer processes occur between 100 fs to $1 \mathrm{~ns}$ after LSPR excitation during which hot carriers are also losing energy through electron-phonon and electron-electron collisions.

Since the energetic hot carriers are generated within the plasmonic NPs and then transferred to the semiconductor, hot carrier injection can occur regardless of the spectral overlap between the Au NPs' LSPR and the contacting semiconductor's absorption spectrum. However, the nature of hot carrier injection as a charge transfer process necessitates contact or a very small tunneling barrier between the plasmonic NPs and semiconductor to which the carriers are being transferred. The hot electrons with sufficient energy to overcome the energetic barrier between plasmonic metal nanoparticle and a contacting semiconductor can be injected into the conduction band of the semiconductor (Figure 2.12a). Similarly, hot holes may also be injected into the valence band of a contacting semiconductor if the hot holes have sufficient energy to overcome the energetic barrier between plasmonic metal and semiconductor (Figure 2.12b). However, since the direction and magnitude of band bending at the interface between the plasmonic metal nanoparticle and contacting semiconductor depends on the difference in Fermi level energies of the plasmonic metals and the contacting semiconductor, As such, hot electrons and hot holes are more effectively 
injected into n-type and p-type semiconductors respectively. Since the hot carriers originate from the LSPR in the plasmonic material and not the semiconductor, hot carrier injection can occur regardless of whether the semiconductor can absorb at the LSPR's excitation wavelengths. However, momentum considerations of the hot electrons and hot holes relative to the semiconductor limit the maximum efficiency of the hot carrier injection process to under $10 \%$ under ideal conditions without special measures to overcome this momentum barrier with current practical transfer efficiencies currently at $1-2 \% . .^{[95,96,109]}$ Nonetheless, the simple requirements for hot carrier injection results in hot carrier injection being a widely used plasmonic enhancement mechanism for improved light harvesting device performance.
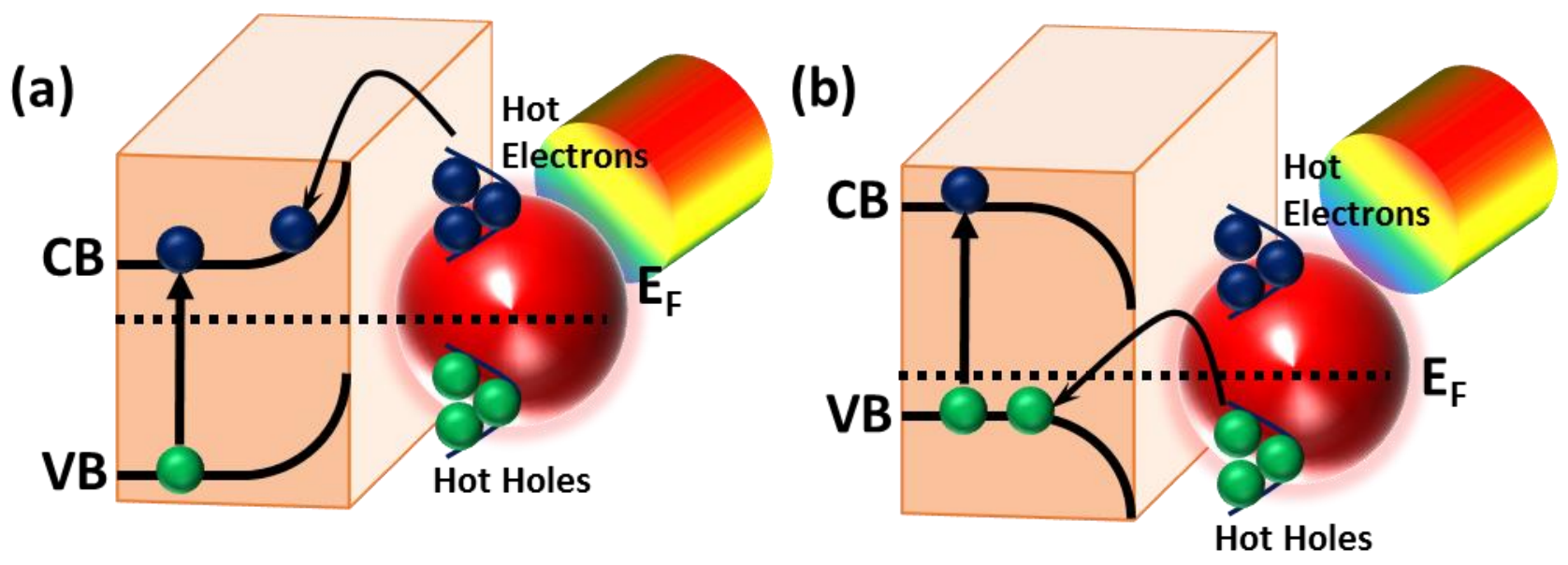

Figure 2.12: Schematic for plasmonic hot carrier injection between plasmonic nanoparticles and semiconductors; (a) hot electron injection into the conduction band of a n-type semiconductor; (b) hot hole injection into the valence band of a p-type semiconductor.

\subsubsection{Non-Plasmonic Effects for Enhancing Photoelectrochemical Cell Performance}

As stated previously, there are non-plasmonic effects that can occur when plasmonic NPs are coupled to semiconductor photoelectrodes. Small plasmonic NPs with diameters or characteristic dimension less than or equal to $10 \mathrm{~nm}$ can be used as electron sinks when deposited on the surface of semiconductor photocatalysts and photoelectrodes. plasmonic NPs' potential to act as electron sinks in due to a phenomenon known as Fermi level equilibration (Figure 2.13) discovered by Dr. Prashant Kamat's group also studying Au NPs on $\mathrm{TiO}_{2}$ in the early 2000 's. ${ }^{[110-}$ ${ }^{112]}$ With Fermi level equilibration, electrons are transferred from the semiconductor into vacant conduction band states within the Au NPs due to quantum mechanics related effects of small $\mathrm{Au}$ NPs. The electron transfer into the surface Au NPs results in a depletion of surface electrons and a corresponding shift in the Fermi level and an increased photovoltage from the semiconductor. The shift in Fermi level can be detected as from a shift in the flat-band potential measured from 
Mott-Schottky (M-S) plots or from illuminated open-circuit voltage measurements. ${ }^{[113]}$ Fermi level equilibration can result in reduced surface charge carrier recombination that can be measured in general from chronoamperometry experiments $(\mathrm{J}-\mathrm{t})^{[114]}$ and an onset potential shift for photocurrent that can lead to improved device efficiencies.

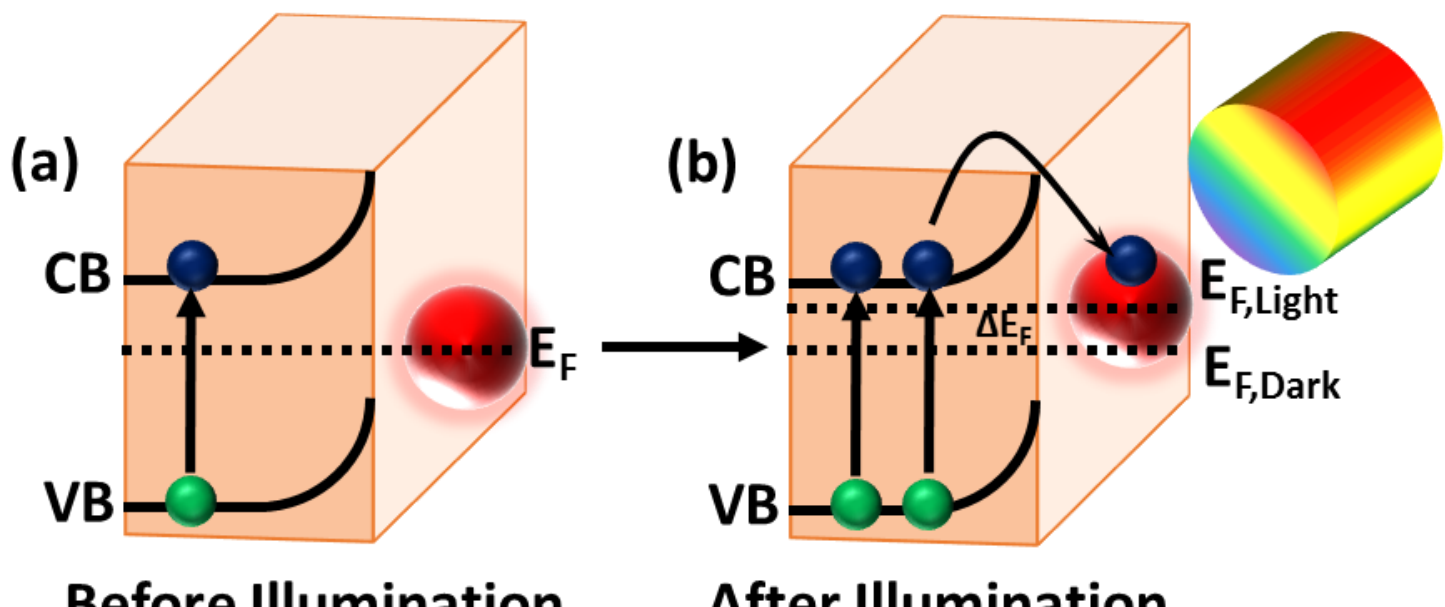

Before Illumination After Illumination

Figure 2.13: Schematic for Fermi level equilibration within a n-type semiconductor as a result of small plasmonic nanoparticles acting as charge carrier sinks upon light illumination.

In addition, plasmonic NPs can be used as electron relays between layers within semiconductor heterostructures. ${ }^{[115]}$ Photoexcited electrons are transferred from one semiconductor to the plasmonic NPs and then transferred from the plasmonic NPs to another semiconductor. By using Au NPs as electron relays, interfacial charge recombination between layers of the semiconductor heterostructure may be suppressed, leading to improved charge transport and overall device conversion efficiencies. Determining whether plasmonic NPs are acting as electron relays is more difficult than testing for Fermi level equilibration. Since the plasmonic NPs are buried and make no contact with the electrolyte, other techniques that can measure charge carrier dynamics are needed to determine whether the Au NPs are acting as electron relays. Ultra-fast transient absorption spectroscopy (TAS) is a useful technique for probing charge carrier dynamics that can be used to determine whether plasmonic NPs are acting as electron relays. In TAS experiments, an ultra-fast laser of fixed wavelength is used to "pump" or excite an initial population of photoexcited charge carriers while a second continuous low intensity light is used to "probe" changes in absorption by the sample across a wide spectrum of wavelengths over femtosecond (fs) to microsecond ( $\mu \mathrm{s})$ timescales. Depending on the pump and probe wavelengths and the materials being studied (i.e. semiconductors with or without plasmonic NPs), charge carrier recombination lifetimes and mechanisms and charge carrier transfer processes can be determined. However, the complex experimental equipment (lasers and optics to generate laser pulses on a fs timescale and physics knowledge required for data analysis unfortunately limits the widespread use of TAS experiments. Since the electron sink and electron relay behaviors do 
not require excitation of the plasmonic NPs' LSPR, the PEC performance enhancement from these mechanisms does not have an illumination wavelength dependence.

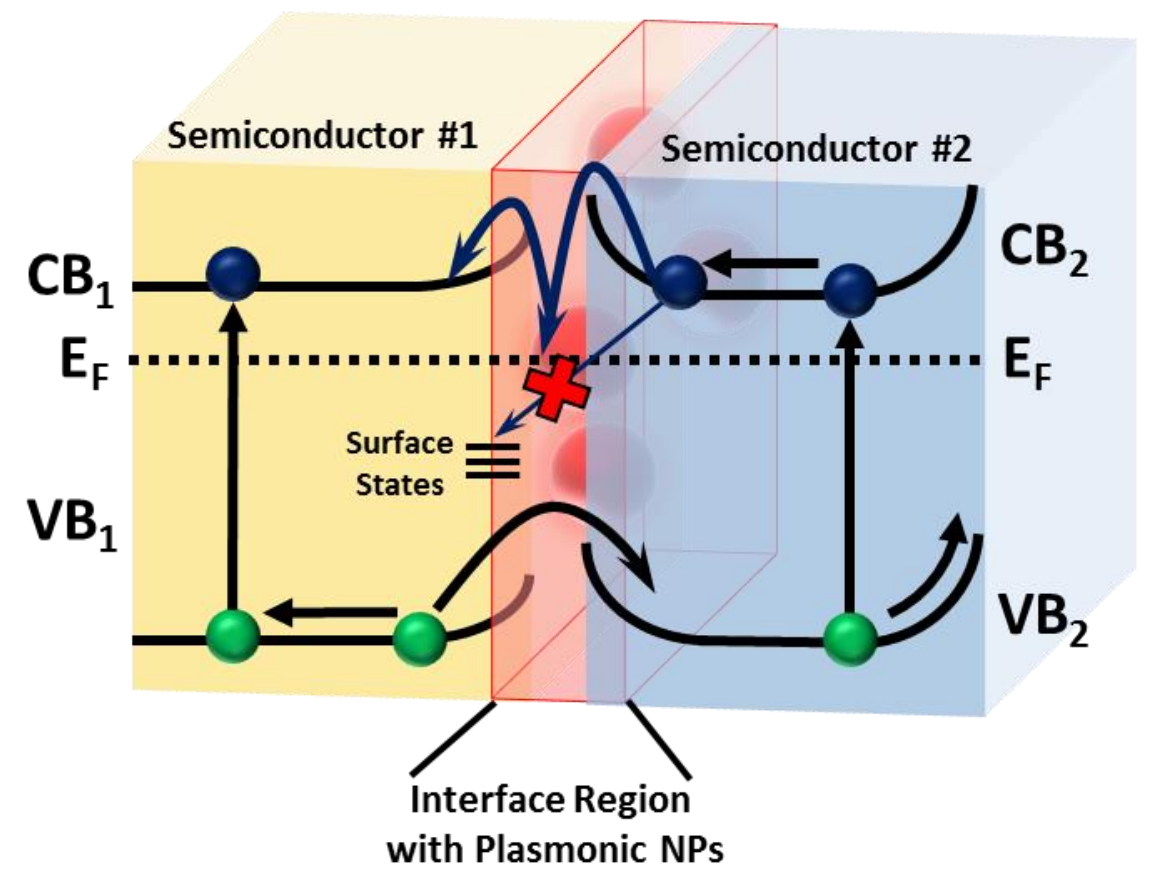

Figure 2.14: Schematic for plasmonic NPs acting as electron relays at the interface of two n-type semiconductors.

In addition, plasmonic NPs in contact with the electrolyte within a PEC cell may also exhibit catalytic activity towards reactions. Small Au NPs can be used as catalysts for driving chemical reactions as shown by Dr. Masatake Haruta's group in the 1985. Ultra-small Au NPs (2$5 \mathrm{~nm}$ ) and $\mathrm{Au}$ atomic clusters on metal oxide and metal hydroxide supports have shown significant catalytic activity towards $\mathrm{CO}$ oxidation, propylene epoxidation, hydrogen dissociation at elevated temperatures. ${ }^{[116,117]}$ Furthermore, Au atomic clusters with significant amounts of exposed high energy edge sites show increased activity show versus larger Au NPs with less edge sites. While $\mathrm{Au}$ NPs are useful for catalyzing some reactions, Au NPs are typically limited as thermally driven catalysts since they typically require elevated temperatures to be effective catalysts. Ultra-small $\mathrm{Au}$ NPs and $\mathrm{Au}$ nanowires with ultra-small cross-sections have been previously used as electrocatalysts for $\mathrm{CO}_{2}$ reduction to $\mathrm{CO} \cdot{ }^{[107,118,119]}$ However, these Au NPs still have issues with morphology stability (i.e. avoiding agglomeration) and reaction selectivity between $\mathrm{CO}$ and $\mathrm{H}_{2}$ generation. In addition, $\mathrm{Cu}$ NPs have shown electrocatalytic activity towards $\mathrm{CO}_{2}$ reduction ${ }^{[120,121]}$ and Ag NPs have shown electrocatalytic activity towards $\mathrm{CO}_{2}$ and $\mathrm{NO}_{3}{ }^{-}$reduction ${ }^{[122]}$. Au NPs can act as weak co-catalysts for water oxidation in highly alkaline electrolytes. ${ }^{[123]}$ However, this catalytic activity is from a thin catalytically active $\mathrm{Au}(\mathrm{OH})_{3}$ layer on the Au NPs formed during operation rather than from the Au NPs themselves. There are more effective co-catalyst options for driving water oxidation such as $\mathrm{Co}-\mathrm{Pi}, \mathrm{FeOOH}$, and $\mathrm{NiOOH} .{ }^{[16,18,41,42]}$ As such, catalysis by $\mathrm{Au}$ NPs is typically a secondary benefit rather than a explicitly sought after design consideration. 
The necessary potential and current required to drive electrochemical reactions can be provided by light energy harvesting devices.

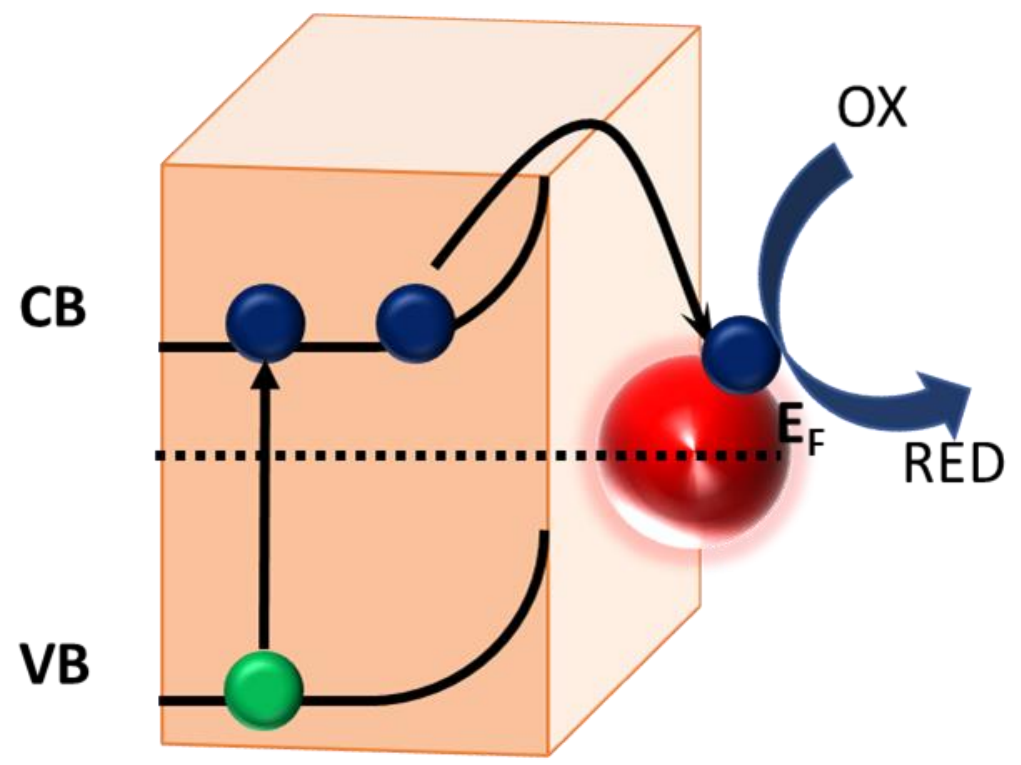

Figure 2.15: Schematic of plasmonic NPs acting as co-catalysts for a n-type semiconductor photoelectrode.

\subsection{Photoelectrochemical (PEC) Biosensors}

Sensors are a device used for detection and quantification of a physical phenomena within an environment. For the purposes of this dissertation, focus will be on sensors that utilize photoelectrochemical (PEC) cells for detection of analytes using biological components. The analyte itself may be a biological or organic in nature or components used to bind the analyte to the photoelectrode may be biological or organic in nature. With PEC biosensors, the measured PEC-based signal such as photovoltage and photocurrent of a photoelectrode is modulated in response to the presence of an analyte. ${ }^{[124-125]}$ The analyte may be contained within the electrolyte of the PEC cell or functionalized onto the surface of the photoelectrode outside of the PEC cell used for testing. The main advantages of PEC sensors are the simple and inexpensive equipment requirements (light source, potentiostat, computer, counter and reference electrodes, and a designed photoelectrode for analyte detection) and simple operation versus other techniques such as SERS. ${ }^{[124-125]}$

In the case of PEC biosensors where the analyte is contained within the electrolyte, the photoelectrode used as the sensing device may directly reduce or oxidize the analyte or may indirectly reduce or oxidize the analyte using a redox mediator that the photoelectrode may reduce or oxidize to then subsequently oxidize or reduce the analyte respectively. However, these two approaches rely on analytes that are electrochemically active species to enable successful detection. In addition, the photoelectrode used in the PEC biosensor must be catalytically active 
towards redox reactions involving the analyte or redox mediator. Selectivity for detection of the analyte is typically achieved by tuning the electrochemical bias used during operation along with tuning the catalytic activity of the photoelectrode to favor redox reactions with the analyte only Examples of PEC biosensors are the $\mathrm{ZnO} / \mathrm{Cu} 2 \mathrm{O}$ p-n junction photoelectrode used for direct detection of glutathione ${ }^{[126]}$. The projects presented within this dissertation do not deal with analytes that are readily electrochemically active. As such, this type of bi all further discussion will focus on PEC sensors that utilize an analyte functionalization step that occurs outside of the PEC cell used for testing.

In the case of PEC biosensors that have the analyte functionalized onto the photoelectrode, the analyte of choice is selectively bound to the photoelectrode. Common methods for binding of analytes include analyte binding to DNA strands ${ }^{[127-131]}$, aptamer-analyte reactions ${ }^{[132-134]}$, and antibody-antigen reactions ${ }^{[135-137]}$. The bound analyte itself may alter the PEC performance of the photoelectrode $^{[132-135,136,138]}$ or a secondary probe may be bound selectively to the photoelectrode at sites where the analyte is already bound ${ }^{[127-131,137,139]}$ to modulate the PEC performance of the photoelectrode. The photoelectrochemical performance of the PEC biosensor's photoelectrode may be modulated utilizing different mechanisms. The following mechanisms are some commonly employed mechanisms for modulating the PEC performance of photoelectrodes in PEC biosensors. It should be noted that more than one of these mechanisms may be combined with each other on a single PEC biosensor.

A commonly employed technique for modulating the PEC performance of photoelectrodes in PEC biosensors is increased charge transfer resistance at the photoelectrode surface due to the presence of bound analyte and bound secondary probes. ${ }^{[132-136,140-143]}$ The presence of biological analytes such as proteins or large probes conjugated to the analyte can impede the diffusion of reactants through steric hinderance to the photoelectrode surface. In addition, the presence of the bound analyte (and bound probes) blocks electrochemically active sites on the photoelectrode surface. The amount of steric hinderance and blocked electrochemically active sites is directly proportional to the concentration of bound analyte. As such, the photocurrent also decreases in a manner that is also proportional to the concentration of bound analyte making this mechanism useful for sensing applications.

Another commonly employed technique for modulating the PEC performance of photoelectrodes in PEC biosensors is the modulation of different energy transfer processes between plasmonic NPs and a semiconductor photoelectrodes. Three different mechanisms, hot electron injection ${ }^{[132-136]}$, PIRET $^{[139]}$, and FRET $^{[127-131,137,144]}$, have been utilized as energy transfer mechanisms. PEC biosensors that utilize hot electron injection have plasmonic nanoparticles already bound to the surface of the semiconductor photoelectrode with analyte binding modulates the magnitude of hot electron injection between the plasmonic nanoparticle and semiconductor photoelectrode. As discussed previously in Section 2.5.1, PIRET and FRET are similar in that they are resonant energy transfer processes that rely on dipole-dipole interactions between plasmonic nanoparticles and semiconductors. However, the direction of energy transfer differs between PIRET and FRET. In PIRET, energy from the LSPR of bound plasmonic nanoparticles is transferred to the semiconductor photoelectrode. The transfer of energy from the plasmonic NPs 
to the semiconductor photoelectrode via PIRET results in increased charge carrier generation and corresponding photocurrent from the semiconductor photoelectrode. To date, there have been few sensors that utilize PIRET from plasmonic NPs in PEC sensors. These PIRET-based PEC sensors utilize selective binding of plasmonic NP probes to semiconductor photoelectrodes in the presence of analyte to initiate PIRET and increase the photocurrent of the photoelectrode. The resulting photocurrent increase is proportional to the amount of bound plasmonic NPs and thus the concentration of analyte used to bind the plasmonic NPs.

In FRET, energy is transferred from the semiconductor to the plasmonic nanoparticle. As such, the loss of energy from the semiconductor photoelectrode results in decreased charge carrier generation and corresponding photocurrent. In the case of FRET-based PEC sensors, the photocurrent of the photoelectrode system may be decreased by selective binding of plasmonic NP probes in the presence of analyte to the photoelectrode surface to initiate $\operatorname{FRET}^{[127-131,135]}$ or increased by selectively increasing the separation distance between the plasmonic nanoparticles and semiconductor photoelectrode to decrease the magnitude of FRET. ${ }^{[144]}$

\subsection{References}

[1] World Energy Council. Solar. World Energy Resources: 2013 Survey, World Energy Council: London, 2013; pp 8.1-8.28.

[2] Jenkins, T. A brief history of ... semiconductors. Phys. Edu., 2005, 40, 430-439.

[3] Ashcroft, N. W.; Mermin, N. D. Homogeneous Semicondcutors. Solid State Physics, Harcourt College Publishers: New York, 1976; pp 561-587.

[4] Muller, R. S.; Kamins, T. I.; Chan, M. Semiconductor Electronics. Device Electronics for Integrated Circuits, John Wiley \& Sons: New York, 2003; pp 2-55.

[5] Bhattacharya, P. Electronic Properties of Semiconductors. Semiconductor Optoelectronic Devices, Prentice Hall: Englewood Cliffs, 1994; pp 59-111.

[6] Bhattacharya, P. Optical Processes in Semiconductors. Semiconductor Optoelectronic Devices, Prentice Hall: Englewood Cliffs, 1994; pp 112-155.

[7] Muller, R. S.; Kamins, T. I.; Chan, M. Currents in pn Junctions. Device Electronics for Integrated Electronics, John Wiley \& Sons: New York, 2003; pp 226-277.

[8] Muller, R. S.; Kamins, T. I.; Chan, M. Silicon Technology. Device Electronics for Integrated Electronics, John Wiley \& Sons: New York, 2003; pp 56-138.

[9] Ashcroft, N. W.; Mermin, N. D. Inhomogeneous Semiconductors. Solid-State Physics Harcourt College Publishers: New York, pp 589-614.

[10] Muller, R. S.; Kamins, T. I.; Chan, M. pn Junctions. Device Electronics for Integrated Circuits, John Wiley \& Sons: New York, 2003; pp. 174-225 
[11] Bhattacharya, P. Solar Cells. Semiconductor Optoelectronic Devices, Prentice Hall: Englewood Cliffs, 1994; pp 413-431.

[12] Fujishima, A.; Honda, K. Electrochemical photolysis of water at a semiconductor electrode. Nature, 1972, 238, 37-38.

[13] Li, J.; Wu, N. Semiconductor-based photocatalysts and photoelectrochemical cells for solar fuel generation: a review. Catal. Sci. Technol., 2015, 5, 1360-1384.

[14] Sivula, K.; van de Krol, R. Semiconducting materials for photoelectrochemical energy conversion. Nat. Rev. Mater., 2016, 1, 15010.

[15] Seabold, J. A.; Zhu, K.; Neale, N. R. Efficient solar photoelectrolysis by nanoporous Mo:BiVO 4 through controlled electron transport. Phys. Chem. Chem. Phys., 2014, 16, 1121-1131.

[16] Pilli, S. K.; Furtak, T. E.; Brown, L. D.; Deutsch, T. G.; Turner, J. A.; Herring, A. M. Cobalt-phosphate (Co-Pi) catalyst modified Mo-doped $\mathrm{BiVO}_{4}$ photoelectrodes for solar water oxidation. Energy Environ. Sci., 2011, 4, 5028-5034.

[17] Du, C.; Yang, X.; Mayer, M. T.; Hoyt, H.; Xie, J.; McMahon, G.; Bischoping, G.; Wang, D. Hematite-based water splitting with low turn-on voltages. Angew. Chem. Intl. Ed., 2013, 52, 12692-12695.

[18] Abdi, F. F.; van de Krol, R. Nature and light dependence of bulk recombination in Co-Picatalyzed $\mathrm{BiVO}_{4}$ photoanodes. J. Phys. Chem. C, 2012, 116, 9398-9404.

[19] Kim, T. W.; Choi, K.-S. Nanoporous $\mathrm{BiVO}_{4}$ photoanode with dual-layer oxygen evolution catalysts for solar water splitting. Science, 2014, 343, 990-994.

[20] Licht, S.; Wang, B.; Mukerji, S.; Soga, T.; Umeno, M.; Tributsch, H. Efficient solar water splitting, exemplified by $\mathrm{RuO}_{2}$-catalyzed AlGaAs/Si photoelectrolysis. J. Phys. Chem. B, 2000, 104, 8920-8924.

[21] Khaselev, O.; Turner, J. A. A monolithic photovoltaic-photoelectrochemical device for hydrogen production via water splitting. Science, 1998, 280, 425-427.

[22] Fountaine, K. T.; Lewerenz, H. J.; Atwater, H. A. Efficiency limits for photoelectrochemical water-splitting. Nat. Commun., 2016, 7, 13706.

[23] Cha, H. G.; Choi, K.-S. Combined biomass valorization and hydrogen production in a photoelectrochemical cell. Nat. Chem., 2015, 7, 328-333.

[24] Wang, G.; Ling, Y.; Wang, H.; Yang, X.;Wang, C.; Zhang, J. Z.; Li, Y. Hydrogen-treated $\mathrm{WO}_{3}$ nanoflakes show enhanced photostability. Energy Environ. Sci., 2012, 5, 61806187.

[25] Zhong, X.; Zhu, Y.; Yang, X.; Wang, S.; Shen, J.; Lin, B.; Li, C. Enhanced visible light photocatalytic activity of interlayer-isolated triplex $\mathrm{Ag} @ \mathrm{SiO}_{2} @ \mathrm{TiO}_{2}$ core-shell nanoparticles. Nanoscale, 2013, 5, 3359-3366. 
[26] Li, Y.; Cheng, X.; Ruan, X.; Song, H.; Lou, Z.; Ye, Z.; Zhu, L. Enhancing photocatalytic activity for visible-light-driven $\mathrm{H}_{2}$ generation with the surface reconstructed $\mathrm{LaTiO}_{2} \mathrm{~N}$ nanostructures. Nano Energy, 2015, 12, 775-784.

[27] Meng, F.; Cushing, S. K.; Li, J.; Hao, S.; Wu, N. Enhancement of solar hydrogen generation by synergistic interaction of $\mathrm{La}_{2} \mathrm{Ti}_{2} \mathrm{O}_{7}$ photocatalyst with plasmonic gold nanoparticles and reduced graphene oxide nanosheets. ACS Catal., 2015, 5, 1949-1955.

[28] Meng, F.; Li, J.; Cushing, S. K.; Zhi, M.; Wu, N. Solar hydrogen generation by nanoscale p$\mathrm{n}$ junction of p-type molybdenum disulfide/n-type nitrogen-doped reduced graphene oxide. J. Am. Chem. Soc., 2013, 135, 10286-10289.

[29] Matsumoto, Y.; Obata, M.;Hombo, J. Photocatalytic reduction of carbon dioxide on p-type $\mathrm{CaFe}_{2} \mathrm{O}_{4}$ powder. J. Phys. Chem., 1994, 98, 2950-2951.

[30] Wang, S.; Hou, Y.; Wang, X. Development of a stable $\mathrm{MnCo}_{2} \mathrm{O}_{4}$ cocatalyst for photocatalytic $\mathrm{CO}_{2}$ reduction with visible light. ACS Appl. Mater. Interfaces, 2015, 7, 4327-4335.

[31] Xu, H.-Q.; Hu, J.; Wang, D.; Li, Z.; Zhang, Q.; Luo, Y.; Yu, S.-H.; Jiang, H.-L. Visiblelight photoreduction of $\mathrm{CO}_{2}$ in a metal-organic framework: Boosting electron-hole separation via electron trap states. J. Am. Chem. Soc., 2015, 137, 13440-13443.

[32] Medford, A. J.; Hatzell, M. C. Photon-driven nitrogen fixation: Current progress, thermodynamic considerations, and future outlook. ACS Catal., 2017, 7, 2624-2643.

[33] Berr, M. J.; Wagner, P.; Fischbach, S.; Vaneski, A.; Schneider, J.; Susha, A. S.; Feldmann, J. Hole scavenger redox potentials determine quantum efficiency and stability of Ptdecorated CdS nanorods for photocatalytic hydrogen generation. Appl. Phys. Lett., 2012, $100,223903$.

[34] Schneider, J.; Bahnemann, D. W. Undesired role of sacrificial reagents in photocatalysis. $J$. Phys. Chem. Lett., 2013, 4, 3479-3483.

[35] Jiang, N.; You, B.; Boonstra, R.; Terrero Rodriguez, I. M.; Sun, Y. Integrating electrocatalytic 5-Hydroxymethylfurfural oxidation and hydrogen production via Co-P derived electrocatalysts. ACS Energy Lett., 2016, 1, 386-390.

[36] You, B.; Liu, X.; Jiang, N.; Sun, Y. A general strategy for decoupled hydrogen production from water splitting by integrating oxidative biomass valorization. J. Am. Chem. Soc., 2016, 138, 13639-13646.

[37] Bloor, L. G.; Solarska, R.; Bienkowski, K.; Kulesza, P. J.; Augustynski, J.; Symes, M. D.; Cronin, L. Solar-driven water oxidation and decoupled hydrogen production mediated by an electron-coupled-proton buffer. J. Am. Chem. Soc., 2016, 138, 6707-6710.

[38] Rausch, B.; Symes, M. D.; Chisholm, G.; Cronin, L. Decoupled catalytic hydrogen evolution from a molecular metal oxide redox mediator in water splitting. Science, 2014, $345,1326-1330$. 
[39] Symes, M. D.; Cronin, L. Decoupling hydrogen and oxygen evolution during electrocatalytic water splitting using an electron-coupled-proton buffer. Nat. Chem., 2013, 5, 403-409.

[40] Rausch, B.; Symes, M. D.; Cronin, L. A bio-inspired, small molecule electron-coupledproton buffer for decoupling the half reactions of electrolytic water splitting. J. Am. Chem. Soc., 2013, 135, 13656-13659.

[41] McCrory, C. C.; Jung, S.; Peters, J. C.; Jaramillo, T. F. Benchmarking heterogeneous electrocatalysts for the oxygen evolution reaction. J. Am. Chem. Soc., 2013, 135, $16977-$ 16987.

[42] Cai, L.; Zhao, J.; Li, H.; Park, J.; Cho, I. S.; Han, H. S.; Zheng, X. One-step hydrothermal deposition of $\mathrm{Ni}$ :FeOOH onto photoanodes for enhanced water oxidation. ACS Energy Lett., 2016, 1, 624-632.

[43] Miller, E. L. Photoelectrochemical Hydrogen Production: DOE PEC Working Group Overview FY 2010. U.S. Department of Energy: Washington D.C., 2011.

[44] Wang, G.; Wang, H.; Ling, Y.; Tang, Y.; Yang, X.; Fitzmorris, R. C.; Wang, C.; Zhang, J. Z.; Li, Y. Hydrogen-treated $\mathrm{TiO}_{2}$ nanowire arrays for photoelectrochemical water splitting. Nano Lett., 2011, 11, 3026-3033.

[45] Wu, N.; Wang, J.; Tafen, D. N.; Wang, H.; Zheng, J.-G.; Lewis, J. P.; Liu, X.; Leonard, S. S.; Manivannan, A. Shape-enhanced photocatalytic activity of single-crystalline anatase $\mathrm{TiO}_{2}$ (101) nanobelts. J. Am. Chem. Soc., 2010, 132, 6679-6685.

[46] Paracchino, A.; Laporte, V.; Sivula, K.; Gratzel, M.; Thimsen, E. Highly active oxide photocathode for photoelectrochemical water reduction. Nat. Mater, 2011, 10, 456-461.

[47] Luo, J.; Steier, L.; Son, M.-K.; Schreier, M.; Mayer, M. T.; Gratzel, M. (2016). $\mathrm{Cu}_{2} \mathrm{O}$ nanowire photocathodes for efficient and durable solar water splitting. Nano Lett., 2016, $16,1848-1857$.

[48] Gao, L.; Cui, Y.; Vervuurt, R. H. J.; van Dam, D.; van Veldhoven, R. P. J.; Hofmann, J. P.; Bol, A. A.; Haverkort, J. E. M.; Notten, P. H. L.; Bakkers, E. P. A. M.; Hensen, E. J. M. High-efficiency InP-based photocathode for hydrogen production by interface energetics design and photon management. Adv. Funct. Mater., 2016, 26, 679-686.

[49] Ye, K.-H.; Li, H.; Huang, D.; Xiao, S.; Qui, W.; Li, M.; Hu, Y.; Mai, W.; Ji, H.; Yang, S. Enhancing photoelectrochemical water splitting by combining work function tuning and heterojunction engineering. Nat. Commun., 2019, 19, 3687.

[50] Dotan, H.; Sivula, K.; Grätzel, M.; Rothschild, A.; Warren, S. C. Probing the photoelectrochemical properties of hematite $\left(\alpha-\mathrm{Fe}_{2} \mathrm{O}_{3}\right)$ electrodes using hydrogen peroxide as a hole scavenger. Energy Environ. Sci., 2011, 4, 958-964. 
[51] Carneiro, L. M.; Cushing, S. K.; Liu, C.; Su, Y.; Yang, P.; Alivisatos, A. P.; Leone, S. R. Excitation-wavelength-dependent small polaron trapping of photoexcited carriers in $\alpha-$ $\mathrm{Fe}_{2} \mathrm{O}_{3}$. Nat. Mater., 2017, 16, 819-826.

[52] Jeon, T. H.; Moon, G.-H.; Park, H.; Choi, W. Ultra-efficient and durable photoelectrochemical water oxidation using elaborately designed hematite nanorod arrays. Nano Energy, 2017, 39, 211-218.

[53] Marin, A. T.; Muñoz-Rojas, D.; Iza, D. C.; Gershon, T.; Musselman, K. P.; MacmanusDriscoll, J. L. Novel atmospheric growth technique to improve both light absorption and charge collection in $\mathrm{ZnO} / \mathrm{Cu}_{2} \mathrm{O}$ thin film solar cells. Adv. Funct. Mater., 2013, 23, 34133419.

[54] Minami, T.; Nishi, Y.; Miyata, T. Impact of incorporating sodium into polycrystalline p-type $\mathrm{Cu}_{2} \mathrm{O}$ for heterojunction solar cell applications. Appl. Phys. Lett., 2014, 105, 212104.

[55] Pan, L.; Kim, J. H.; Mayer, M. T.; Son, M.-K.; Ummadisingu, A.; Lee, J. S.; Hagfeldt, A.; Luo, J.; Grätzel, M. Boosting the performance of $\mathrm{Cu}_{2} \mathrm{O}$ photocathodes for unassisted solar water splitting devices. Nat. Catal., 2018, 1, 412-420.

[56] Furukawa, H.; Cordova, K. E.; O'Keeffe, M.; Yaghi, O. M. The chemistry and applications of metal-organic-frameworks. Science, 2013, 341, 1230444.

[57] Zeng, L.; Guo, X.; He, C.; Duan, C. Metal-organic frameworks: Versatile materials for heterogeneous photocatalysis. ACS Catal., 2016, 6, 7935-7947.

[58] Nguyen, H. L. The chemistry of titanium-based metal-organic frameworks. New J. Chem., 2017, 41, 14030-14043.

[59] Fu, Y.; Sun, D.; Chen, Y.; Huang, R.; Ding, Z.; Fu, X.; Li, Z. An amine-functionalized titanium metal-organic framework photocatalyst with visible-light-induced activity for $\mathrm{CO}_{2}$ reduction. Angew. Chem., 2012, 124, 3420-3423.

[60] Gao, J.; Miao, J.; Li, P.-Z.; Teng, W. Y.; Yang, L.; Zhao, Y.; Liu, B.; Zhang, Q. A p-type Ti(IV)-based metal-organic framework with visible-light photoresponse. Chem. Commun. 2014, 50, 3786-3788.

[61] Fateeva, A.; Chater, P. A.; Ireland, C. P.; Tahir, A. A.; Khimyak, Y. Z.; Wiper, P. V.; Rosseinsky, M. J. A water-stable porphyrin-based metal-organic framework active for visible light photocatalysis. Angew. Chem., 2012, 124, 7558-7562.

[62] Park, H. J.; So, M. C.; Gosztola, D.; Wiederrecht, G. P.; Emery, J. D.; Martinson, A. B.; Er, S.; Wilmer, C. E.; Vermeulen, N. A.; Aspuru-Guzik, A.; Stoddart, J. F.; Farha, O. K.; Hupp, J. T. Layer-by-layer assembled films of perylene diimide- and squaraine containing metal-organic framework-like materials: Solar energy capture and directional energy transfer. ACS Appl. Mater. Inter., 2016, 8, 24983-24988. 
[63] Sun, D.; Fu, Y.; Liu, W.; Ye, L.; Wang, D.; Yang, L.; Fu, X.; Li, Z. Studies on photocatalytic $\mathrm{CO}_{2}$ reduction over $\mathrm{NH}_{2}-\mathrm{Uio}-66(\mathrm{Zr})$ and its derivatives: Towards a better understanding of photocatalysis on metal-organic frameworks. Chem. Eur. J., 2013, 19, $14279-14285$.

[64] Shen, L.; Liang, R.; Luo, M.; Jing, F.; Wu, L. Electronic effects of ligand substitution on metal-organic framework photocatalysts: the case study of UiO-66. Phys. Chem. Chem. Phys., 2015, 16, 117-121.

[65] Wang, A.; Zhou, Y.; Wang, Z.; Chen, M.; Sun, L.; Liu, X. Titanium incorporated with UiO-66(Zr)-type metal-organic framework (MOF) for photocatalytic application. RSC $A d v .$, 2016, 6, 3671-3679.

[66] Li, J.; Musho, T.; Bright, J.; Wu, N. Functionalization of a metal-organic framework semiconductor for tuned band structure and catalytic activity. J. Electrochem. Soc., 2019, 166, H3029-H3034.

[67] Shen, L.; Wu, W.; Liang, R.; Lin, R.; Wu, L. Highly dispersed palladium nanoparticles anchored on UiO-66 $\left(\mathrm{NH}_{2}\right)$ metal-organic framework as a reusable and dual functional visible-light-driven photocatalyst. Nanoscale, 2013, 5, 9374-9382.

[68] Wang, R.; Gu, L.; Zhou, J.; Liu, X.; Teng, F.; Li, C.; Shen, Y.; Yuan, Y. Quasipolymeric metal-organic framework $\mathrm{UiO}-66 / \mathrm{g}-\mathrm{C}_{3} \mathrm{~N}_{4}$ heterojunctions for enhanced photocatalytic hydrogen evolution under visible light irradiation. Adv. Mater. Interfaces, 2015, 2, 1500037.

[69] Zhan, W.; Kuang, Q.; Zhou, J.; Kong, X. Xie, Z.; Zheng, L. Semiconductor@ metal-organic framework core-shell heterostructures: A case of ZnO@ZIF-8 nanorods with selective photoelectrochemical response. J. Am. Chem. Soc., 2013, 135, 1926-1933.

[70] Liu, Q.; Low, Z.-X.; Li, L.; Razmjou, A.; Wang, K.; Yao, J.; Wang, H. ZIF-8/Zn2 $\mathrm{GeO}_{4}$ nanorods with an enhanced $\mathrm{CO}_{2}$ adsorption property in an aqueous medium for photocatalytic synthesis of liquid fuel. J. Mater. Chem. A, 2013, 1, 11563-11569.

[71] Wang, X.; Liu, J.; Leong, S.; Lin, X.; Wei, J.; Kong, B.; Xu, Y.; Low, Z.-X.; Yao, J.; Wang, H. Rapid construction of ZnO@ZIF-8 heterostructures with size-selective photocatalysis properties. ACS Appl. Mater. Interfaces, 2016, 8, 9080-9087.

[72] Jing, H.-P.; Wang, C.-C.; Zhang, Y.-W.; Wang, P.; Li, R. Photocatalytic degradation of methylene blue in ZIF-8. RSC Adv., 2014, 4, 54454-54462.

[73] Zeng, X.; Huang, L.; Wang, C.; Wang, J.; Li, J.; Luo, X. Sonocrystallization of ZIF-8 on electrostatic spinning $\mathrm{TiO}_{2}$ nanofibers surface with enhanced photocatalysis property through synergistic effect. ACS Appl. Mater. Interfaces, 2016, 8, 20274-20282.

[74] Isimjan, T. T.; Kazemian, H.; Rohani, S.; Ray, A. K. Photocatalytic activities of Pt/ZIF-8 loaded highly ordered $\mathrm{TiO}_{2}$ nanotubes. J. Mater. Chem., 2010, 20, 10241-10245. 
[75] Kaur, R.; Rana, A.; Singh, R. K.; Chhabra, V. A.; Kim, K.-H.; Deep, A. Efficient photocatalytic and photovoltaic applications with nanocomposites between CdTe QDs and an NTU-9 MOF. RSC Adv., 2017, 7, 29015-29024.

[76] Zhang, L.; Cui, P.; Yang, H.; Chen, J.; Xiao, F.; Guo, Y.; Liu, Y.; Zhang, W.; Huo, F.; Liu, B. Metal-organic frameworks as promising photosensitizers for photoelectrochemical water splitting. Adv. Sci., 2016, 3, 1500243.

[77] Sun, D.; Liu, W.; Fu, Y.; Fang, Z.; Sun, F.; Fu, X.; Zhang, Y.; Li, Z. Noble metals can have different effects on photocatalysis over metal-organic frameworks (MOFs): A case study on M/NH2-MIL-125(Ti) (M=Pt and Au). Chem. Eur. J., 2014, 20, 4780-4788.

[78] Chambers, M. B.; Wang, X.; Ellezam, L.; Ersen, O.; Fontecave, M.; Sanchez, C.; Rozes, L.; Mellot-Draznieks, C. Maximizing the photocatalytic activity of metal-organic frameworks with aminated-functionalized linkers: Substoichiometric effects in MIL-125$\mathrm{NH}_{2}$.

[79] Feng, D.; Gu, Z.-Y.; Li, J.-R.; Jiang, H.-L.; Wei, Z.; Zhou, H.-C. Zirconiummetalloporphyrin PCN-222: Mesoporous metal-organic frameworks with ultrahigh stability as biomimetic catalysts. Angew. Chem. Int. Ed., 2012, 51, 10307-10310.

[80] Xu, H.-Q.; Hu, J.; Wang, D.; Li, Z.; Zhang, Q.; Luo, Y.; Yu, S.-H.; Jiang, H.-L. Visiblelight photoreduction of $\mathrm{CO}_{2}$ in a metal-organic framework: Boosting electron-hole separation via electron trap states. J. Am. Chem. Soc., 2015, 137, 13440-13443.

[81] Feng, D.; Gu, Z.-Y.; Chen, Y.-P.; Park, J.; Wei, Z.; Sun, Y.; Bosch, M.; Yuan, S.; Zhou, H.-C. A highly stable porphyrinic zirconium metal-organic framework with shp-a topology. J. Am. Chem. Soc., 2014, 136, 17714-17717.

[82] Feng, D.; Chung, W.-C.; Wei, Z.; Gu, Z.-Y.; Jiang, H.-L.; Chen, Y.-P.; Darensbourg, D. J.; Zhou, H.-C. Construction of ultrastable porphyrin Zr metal-organic frameworks through linker elimination. J. Am. Chem. Soc., 2013, 135, 17105-17110.

[83] Jiang, H.-L.; Feng, D.; Wang, K.; Gu, Z.-Y.; Wei, Z.; Chen, Y.-P.; Zhou, H.-C. An exceptionally stably, porphyrinic $\mathrm{Zr}$ metal-organic framework exhibiting $\mathrm{pH}$-dependent fluorescence. J. Am. Chem. Soc., 2013, 135, 13934-13938.

[84] Willets, K. A.; Van Duyne, R. P. Localized surface plasmon resonance spectroscopy and sensing. Annu. Rev. Phys. Chem., 2007, 58, 267-297.

[85] Maier, S. A.; Atwater, H. A. Plasmonics: Localization and guiding of electromagnetic energy in metal/dielectric structures. J. Appl. Phys., 2005, 98, 011101.

[86] Wu, N. Plasmonic metal-semiconductor photocatalysts and photoelectrochemical cells: a review. Nanoscale, 2018, 10, 2679-2696.

[87] Clavero, C. Plasmon-induced hot electron generation at nanoparticle/metal-oxide interfaces for photovoltaic and photocatalytic devices. Nat. Photon., 2014, 8, 95-103. 
[88] Cushing, S. K.; Wu, N. Plasmon-enhanced solar energy harvesting. Interface, 2013, 63-67.

[89] Cushing, S. K.; Li, J.; Meng, F.; Senty, T. R.; Suri, S.; Zhi, M.; Li, M.; Bristow, A. D.; Wu, N. Photocatalytic activity enhanced by plasmonic resonant energy transfer from metal to semiconductor. J. Am. Chem. Soc., 2012, 134, 15033-15041.

[90] Li, J.; Cushing, S. K.; Meng, F.; Senty, T. R.; Bristow, A. D.; Wu, N. Plasmon-induced resonance energy transfer for solar energy conversion. Nat. Photon., 2015, 9, 601-607.

[91] Meng, F.; Cushing, S. K.; Li, J.; Hao, S.; Wu, N. Enhancement of solar hydrogen generation by synergistic interaction of $\mathrm{La}_{2} \mathrm{Ti}_{2} \mathrm{O}_{7}$ photocatalyst with plasmonic gold nanoparticles and reduced graphene oxide nanosheets. ACS Catal., 2015, 5, 1949-1955.

[92] Zhong, X.; Zhu, Y.; Yang, X.; Wang, S.; Shen, J.; Lin, B.; Li, C. Enhanced visible light photocatalytic activity of interlayer-isolated triplex Ag@ $\mathrm{SiO}_{2} @ \mathrm{TiO}_{2}$ core-shell nanoparticles. Nanoscale, 2013, 5, 3359-3366.

[93] Li, J., Cushing, S. K., Bright, J., Meng, F., Senty, T. R., Zheng, P., Bristow, A. D.; Wu, N. $\mathrm{Ag} @ \mathrm{Cu}_{2} \mathrm{O}$ core-shell nanoparticles as visible-light plasmonic photocatalysts. ACS Catal., 2013, 3, 47-51.

[94] Li, J.; Cushing, S. K.; Zheng, P.; Meng, F.; Chu, D.; Wu, N. Plasmon-induced photonic and energy-transfer enhancement of solar water splitting by a nanorod array. Nat. Commun., 2013, 4, 2651.

[95] Cushing, S. K.; Bristow, A. D.; Wu, N. Theoretical maximum efficiency of solar energy conversion in plasmonic metal-semiconductor heterojunctions. Phys. Chem. Chem. Phys., 2015, 17, 30013-30022.

[96] Cushing, S. K.; Wu, N. Progress and perspectives of plasmon-enhanced solar energy conversion. J. Phys. Chem. Lett., 2016, 7, 666-675.

[97] Cushing, S. K.; Li, J.; Bright, J.; Yost, B. T.; Zheng, P.; Bristow, A. D.; Wu, N. Controlling plasmon-induced resonance energy transfer and hot electron injection processes in metal@ $\mathrm{TiO}_{2}$ core-shell nanoparticles. J. Phys. Chem. C, 2015, 119, 16239-16244.

[98] Link, S.; El-Sayed, M. A. Size and temperature dependence of the plasmon absorption of colloidal gold nanoparticles. J. Phys. Chem. B, 1999, 103, 4212-4217.

[99] Li, M.; Cushing, S. K.; Zhang, J.; Lankford, J.; Aguilar, Z. P.; Ma, D.; Wu, N. Shapedependent surface-enhanced Raman scattering in gold-Raman-probe-silica sandwiched nanoparticles for biocompatible applications. Nanotechnology, 2012, 23, 115501.

[100] DuChene, J. S.; Williams, B. P.; Johnston-Peck, A. C.; Qiu, J.; Gomes, M.; Amilhau, M.; Bejleri, D.; Weng, J.; Su, D.; Huo, F.; Stach, E. A.; Wei, W. D. Elucidating the sole contribution from electromagnetic near-fields in plasmon-enhanced $\mathrm{Cu}_{2} \mathrm{O}$ photocathodes. Adv. Energy Mater., 2016, 6, 1501250. 
[101] Klar, T.; Perner, M.; Grosse, S.; von Plessen, G.; Spirkl, W.; Feldmann, J. Surfaceplasmon resonances in single metallic nanoparticles. Phys. Rev. Lett., 1998, 80, 42494252 .

[102] Schaadt, D. M.; Feng, B.; Yu, E. T. Enhanced semiconductor optical absorption via surface plasmon excitation in metal nanoparticles. Appl. Phys. Lett., 2005, 86, 063106.

[103] Derkacs, D.; Lim, S. H.; Matheu, P.; Mar, W.; Yu, E. T. Improved performance of amorphous silicon solar cells via scattering from surface plasmon polaritons in nearby metallic nanoparticles. Appl. Phys. Lett., 2006, 89, 093103.

[104] Tian, Y.; Tatsuma, T. Plasmon-induced photoelectrochemistry at metal nanoparticles supported on nanoporous $\mathrm{TiO}_{2}$. Chem. Commun., 2004, 1810-1811.

[105] Yu, K.; Tian, Y.; Tatsuma, T. Size effects of gold nanaoparticles on plasmon-induced photocurrents of gold- $\mathrm{TiO}_{2}$ nanocomposites. Phys. Chem. Chem. Phys., 2006, 8, 54175420 .

[106] Nishi, H.; Sakamoto, M.; Tatsuma, T. Local trapping of energetic holes at gold nanoparticles on $\mathrm{TiO}_{2}$. Chem. Commun., 2018, 54, 11741-11744.

[107] DuChene, J. S.; Tagliabue, G.; Welch, A. J.; Cheng, W.-H.; Atwater, H. A. Hot hole collection and photoelectrochemical $\mathrm{CO}_{2}$ reduction with plasmonic $\mathrm{Au} / \mathrm{p}-\mathrm{GaN}$ photocathodes. Nano Lett., 2018, 18, 2545-2550.

[108] Mubeen, S.; Hernandez-Sosa, G.; Moses, D.; Lee, J.; Moskovits, M. Plasmonic photosensitization of a wide band gap semiconductor: Converting plasmons to charge carriers. Nano Lett., 2011, 11, 5548-5552.

[109] Leenheer, A. J.; Narang, P.; Lewis, N. S.; Atwater, H. A. Solar energy conversion via hot electron internal photoemission in metallic nanostructures: Efficiency estimates. J. Appl. Phys., 2014, 115, 134301.

[110] Subramanian, V.; Wolf, E. E.; Kamat, P. V. Catalysis with $\mathrm{TiO}_{2} /$ gold nanocomposites. Effect of metal particle size on the Fermi level equilibration. J. Am. Chem. Soc., 2004, $126,4943-4950$.

[111] Chandrasekharan, N.; Kamat, P. V. Improving the photoelectrochemical performance of nanostructured $\mathrm{TiO}_{2}$ films by adsorption of gold nanoparticles. J. Phys. Chem. B, 2000, 104, 10851-10857.

[112] Subramanian, V.; Wolf, E.; Kamat, P. V. Semiconductor-metal composite nanostructures. To what extent do metal nanoparticles improve the photocatalytic activity of $\mathrm{TiO}_{2}$ films? J. Phys. Chem. B, 2001, 105, 11439-11446.

[113] Chen, Z.; Dinh, H. N.; Miller, E. Flat-band potential techniques. Photoelectrochemical Water Splitting: Standards, Experimental Methods, and Protocols, Springer Briefs in Energy; Springer: New York, 2013; pp 63-84. 
[114] Tafalla, D.; Salvador, P.; Benito, R. M. Kinetic approach to the photocurrent transients in water photoelectrolysis at $\mathrm{n}-\mathrm{TiO}_{2}$ electrodes II. Analysis of the photocurrent-time dependence. J. Electrochem. Soc., 1990, 137, 1810-1815.

[115] Li, J.; Cushing, S. K.; Zheng, P.; Senty, T.; Meng, F.; Bristow, A. D.; Manivannan, A.; $\mathrm{Wu}, \mathrm{N}$. Solar hydrogen generation by a $\mathrm{CdS}-\mathrm{Au}-\mathrm{TiO}_{2}$ sandwich nanorod array enhanced with Au nanoparticle as electron relay and plasmonic photosensitizer. J. Am. Chem. Soc., 2014, 136, 8438-8449.

[116] Haruta, M., When gold is not noble: Catalysis by nanoparticles. Chem. Rec., 2003, 3, 7587

[117] Haruta, M., Role of perimeter interfaces in catalysis by gold nanoparticles. Faraday Discuss., 2011, 152, 11-32.

[118] Trindell, J. A.; Clausmeyer, J.; Crooks, R. M. Size stability and $\mathrm{H}_{2} / \mathrm{CO}$ selectivity for Au nanoparticles during electrocatalytic $\mathrm{CO}_{2}$ reduction. J. Am. Chem. Soc., 2017, 139, 16161-16167.

[119] Zhu, W.; Zhang, Y.-J.; Zhang, H.; Lv, H.; Li, Q.; Michalsky, R.; Peterson, A. A.; Sun, S. Active and selective conversion of $\mathrm{CO}_{2}$ to $\mathrm{CO}$ on ultrathin Au nanowires. J. Am. Chem. Soc., 2014, 136, 16132-16135.

[120] Gao, D.; Zegkinoglou, I.; Divins, N. J.; Scholten, F.; Sinev, I.; Grosse, P.; Roldan Cuenya, B. Plasma-activated copper nanocube catalysts for efficient carbon dioxide electroreduction to hydrocarbons and alcohols. ACS Nano, 2017, 11, 4825-4831.

[121] Reske, R.; Mistry, H.; Behafarid, F.; Roldan Cuenya, B.; Strasser, P. Particle size effects in the catalytic electroreduction of $\mathrm{CO}_{2}$ on $\mathrm{Cu}$ nanoparticles. J. Am. Chem. Soc., 2014, 136, 6978-6986.

[122] Kim, Y.; Creel, E. B.; Corson, E. R.; McCloskey, B. D.; Urban, J. J.; Kostecki, R. Surfaceplasmon assisted photoelectrochemical reduction of $\mathrm{CO}_{2}$ and $\mathrm{NO}_{3}{ }^{-}$on nanostructured silver electrodes. Adv. Energy Mater., 2018, 8, 1800363.

[123] Koren, M. G.; Dotan, H.; Rothschild, A. Nano gold rush: On the origin of the photocurrent enhancement in hematite photoanodes decorated with gold nanoparticles. J. Phys. Chem. $C, \mathbf{2 0 1 6}, 120,15042-15051$.

[124] Zhao, W.-W., Xu, J.-J., \& Chen, H.-Y. Photoelectrochemical bioanalysis: The state of the art. Chem. Soc. Rev., 2015, 44, 729-741.

[125] Zhang, X., Guo, Y., Liu, M., \& Zhang, S. Photoelectrocehmically active species and photoelectrochemical biosensors. RSC Adv., 2013, 3, 2846-2857.

[126] Kang, Z.; Yan, X.; Wang, Y.; Bai, Z.; Liu, Y.; Zhang, Z.; Lin, P.; Zhang, X.; Yuan, H.; Zhang, X.; Zhang, Y. Electronic structure engineering of $\mathrm{Cu}_{2} \mathrm{O} / \mathrm{ZnO}$ nanorods array alloxide $\mathrm{p}$-n heterostructure for enhanced photoelectrochemical property and self-powered biosensing application. Sci. Rep., 2015, 5, 7882. 
[127] Zhao, W.-W.; Yu, P.-P.; Shan, Y.; Wang, J.; Xu, J.-J.; Chen, H.-Y. Exciton-plasmon interactions between $\mathrm{CdS}$ quantum dots and $\mathrm{Ag}$ nanoparticles in photoelectrochemical system and its biosensing application. Anal. Chem., 2012, 84, 5892-5897.

[128] Han, D.-M.; Jiang, L.-Y.; Tang, W.-Y.; Xu, J.-J.; Chen, H.-Y. Photoelectrochemical determination of inorganic mercury ions based on energy transfer between CdS quantum dots and Au nanoparticles. Electrochem. Commun., 2015, 51, 72-75.

[129] Dong, Y.-X.; Cao, J.-T.; Wang, B.; Ma, S.-H.; Liu, Y.-M. Exciton-plasmon interactions between $\mathrm{CdS} @ \mathrm{~g}-\mathrm{C}_{3} \mathrm{~N}_{4}$ heterojunction and $\mathrm{Au} @ \mathrm{Ag}$ nanoparticles coupled with DNAasetriggered signal amplification: Toward highly sensitive photoelectrochemical bioanalysis of microRNA. ACS Sustainable Chem. Eng., 2017, 5, 10840-10848.

[130] Shi, X.-M.; Mei, L.-P.; Wang, Q.; Zhao, W.-W.; Xu, J.-J.; Chen, H.-Y. Energy transfer between semiconducting polymer dots and gold nanoparticles in a photoelectrochemical system: A case application for cathodic bioanalysis. Anal. Chem., 2018, 90, 4277-4281.

[131] Dong, Y.-X.; Cao, J.-T.; Liu, Y.-M.; Ma, S.-H. A novel immunosensing platform for highly sensitive prostate specific antigen detection based on dual-quenching of photocurrent from $\mathrm{CdSe}$ sensitized $\mathrm{TiO}_{2}$ electrode by gold nanoparticles decorated polydopamine nanospheres. Biosens. Bioelectron., 2017, 91, 246-252.

[132] Da, P.; Li, W.; Lin, X.; Wang, Y.; Tang, J.; Zheng, G. Surface plasmon resonance enhanced real time photoelectrochemical protein sensing by gold nanoparticle-decorated $\mathrm{TiO}_{2}$ nanowires. Anal. Chem., 2014, 86, 6633-6639.

[133] Xin, Y.; Li, Z.; Zhang, Z. Photoelectrochemical aptasensor for the sensitive and selective detection of kanamycin based on Au nanoparticle functionalized self-doped $\mathrm{TiO}_{2}$ nanotube arrays. Chem. Commun., 2015, 51, 15498-15501.

[134] Qiao, Y.; Li, J.; Li, H.; Fang, H.; Fan, D.; Wang, W. A label-free photoelectrochemical aptasensor for bisphenol A based on surface plasmon resonance of gold nanoparticlesensitized $\mathrm{ZnO}$ nanopencils. Biosens. Bioelectron., 2016, 86, 315-320.

[135] Zhu, Y.-C.; Zhang, N.; Ruan, Y.-F.; Zhao, W.-W.; Xu, J.-J.; Chen, H.-Y. Alkaline phosphatase tagged antibodies on gold nanoparticles/ $/ \mathrm{TiO}_{2}$ nanotubes electrode: A plasmonic strategy for label-fee and amplified photoelectrochemical immunoassay. Anal. Chem., 2016, 88, 5626-5630.

[136] Shi, Y.; Zhang, Q.; Zhai, T.-T.; Zhou, Y.; Yang, D.-R.; Wang, F.-B.; Xia, X.-H. Localized surface plasmon resonance enhanced label-free photoelectrochemical immunoassay by $\mathrm{Au}-\mathrm{MoS}_{2}$ nanohybrid. Electrochim. Acta, 2018, 271, 361-369.

[137] Wang, Y.; Yu, X.; Ye, X.; Wu, K.; Wu, T.; Li, C. Resonance energy transfer between $\mathrm{ZnCdHgSe} \mathrm{quantum} \mathrm{dots} \mathrm{and} \mathrm{gold} \mathrm{nanorods} \mathrm{enhancing} \mathrm{photoelectrochemical}$ immunosensing of prostate specific antigen. Anal. Chim. Acta, 2016, 943, 106-113. 
[138] Wang, S.-S.; Zhao, X.-P.; Liu, F.-F.; Younis, M. R.; Xia, X.-H.; Wang, C. Direct plasmon-enhanced electrochemistry for enabling ultrasensitive and label-free detection of circulating tumor cells in blood. Anal. Chem., 2019, 91, 4413-4420.

[139] Shu, J.; Qiu, Z.; Lv, S.; Zhang, K.; Tang, D. Plasmonic enhancement coupling with defectengineered $\mathrm{TiO}_{2-\mathrm{x}}$ : A mode for sensitive photoelectrochemical biosensing. Anal. Chem., 2018, 90, 2425-2429.

[140] Liu, Y; Yan, T.; Li, Y.; Cao, W.; Pang, X.; Wu, D.; Wei, Q. A simple label-free photoelectrochemical immunosensor for highly sensitive detection of aflatoxin $\mathrm{B} 1$ based on $\mathrm{CdS}-\mathrm{Fe}_{3} \mathrm{O}_{4}$ magnetic nanocomposites. RSC Adv., 2015, 5, 19581-19586.

[141] Wang, Y.; Fan, D.; Zhao, G.; Feng, J.; Wei, D.; Zhang, N.; Cao, W.; Du, B.; Wei, Q. Ultrasensitive photoelectrochemical immunosensor for the detection of amyloid $\beta$-protein based on $\mathrm{SnO}_{2} / \mathrm{SnS}_{2} / \mathrm{Ag}_{2} \mathrm{~S}$ nanocomposites. Biosens. Bioelectron., 2018, 120, 1-7.

[142] Zhang, Y.; Liu, Y.; Li, R.; Khan, M. S.; Gao, P.; Zhang, Y.; Wei, Q. Visible-light driven photoelectrochemical immunosensor based on $\mathrm{SnS}_{2} @ \mathrm{mpg}-\mathrm{C}_{3} \mathrm{~N}_{4}$ for detection of prostate specific antigen. Sci. Rep., 2017, 7, 4629.

[143] Fan, D.; Wu, D.; Cui, J.; Chen, Y.; Ma, H.; Liu, Y.; Wei, Q.; Du, B. An ultrasensitive label-free immunosensor based on $\mathrm{CdS}$ sensitized $\mathrm{Fe}-\mathrm{TiO}_{2}$ with high visible-light photoelectrochemical activity. Biosens. Bioelectron., 2015, 74, 843-848.

[144] Liu, S.; Cao, H.; Wang, X.; Tu, W.; Dai, Z. Green light excited ultrasensitive photoelectrochemical biosensing for microRNA at a low applied potential based on the dual role of Au NPs in $\mathrm{TiO}_{2}$ nanorods/Au NPs composites. Nanoscale, 2018, 10, 1647416478. 


\section{Chapter 3: Porphyrin-based Metal-Organic Framework Coated Titanium Dioxide Nanorod Array for Improved Photoelectrochemical Cell Performance}

\subsection{Introduction}

Fossil fuels currently provide for most of the world's energy needs. However, consumption of fossil fuels releases pollutants and greenhouse gases that cause global climate change. Therefore, new sustainable energy sources are needed to meet the world's increasing energy demand. Solar fuels generated through photoelectrochemical or photocatalytic water-splitting, which represents a sustainable energy source ${ }^{[1-8]}$. Unfortunately, commercialization of semiconductor-based photocatalysts and photoelectrochemcial cells (PECs) is still hindered by low solar-to-chemical energy conversion efficiency due to the lack of semiconductors with sufficient sunlight absorption, charge transport properties and catalytic activity for watersplitting ${ }^{[9]}$. Titanium dioxide $\left(\mathrm{TiO}_{2}\right)$ is a commonly used photocatalyst and photoelectrode material due to its low cost, favorable valence band energetics for water oxidation, ease in forming nanostructures (i.e. nanorods) and photoelectrochemical stability in aqueous electrolytes ${ }^{[10]}$. However, $\mathrm{TiO}_{2}$ has several problems such as a high density of surface trap-states, poor catalytic activity for water oxidation and poor sunlight absorption due to its wide bandgap, which limits its efficiency for solar-water splitting.

Metal-organic frameworks (MOFs) are a class of porous inorganic-organic hybrid materials built with metal-oxo clusters bridged by organic ligands, resulting in a three-dimensional (3D) porous structure ${ }^{[11]}$. MOFs can be constructed with various ligands and metal centers as the molecular building blocks, which provides great flexibility for tuning chemical and physical properties $^{[12-14]}$. They have the highest specific surface area and pore volume among all porous materials, proved to be a superior platform for immobilization of molecular catalysts on conductive substrates; can be tuned to have high selectivity towards a specific chemical reaction. Theoretical calculation predicts that the optical band gap of MOF semiconductors can be tuned from 1.0 to 5.5 $\mathrm{eV}^{[14]}$. Therefore, MOFs are appealing candidates for photocatalysts ${ }^{[15]}$. The application of MOFs in photoelectrochemical cells as photoelectrodes is rare although particulate MOF photocatalysts have been reported ${ }^{[1,12,16-20]}$. The photoelectrochemical water splitting behavior of MOFs remains poorly understood.

In this chapter, a thin, uniform and conformal p-type porphyrin-based MOF, known as PCN-225 (Fig. 3.1) is coated on the surface of a n-type $\mathrm{TiO}_{2}$ nanorod array through a layer-bylayer (LbL)self-assembly method as shown in Fig. 3.2. TCPP (TCPP = tetrakis(4carboxyphenyl)porphyrin) was employed as a ligand; and highly stable Zr6 clusters were chosen as nodes for the assembly of MOFs. A porphyrinic MOF is selected as the test material because of 
its high stability in aqueous solutions favorable band gap and relatively high electronic conductivity compared to other MOF counterparts ${ }^{[21-27]}$.

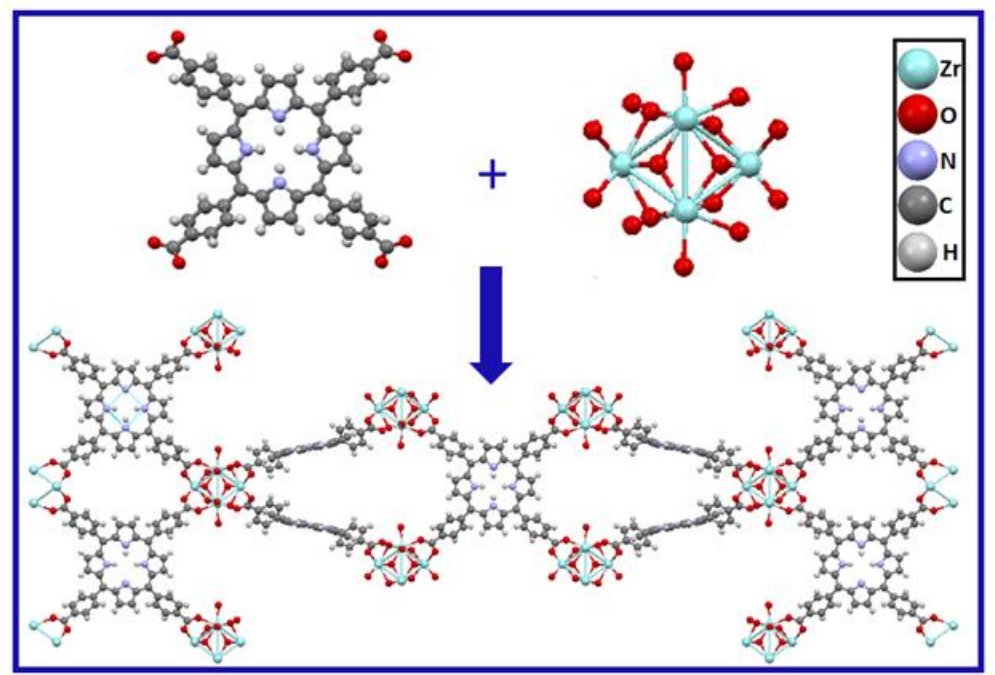

Figure 3.1: Chemical structure and synthetic route of the PCN-225 MOF.

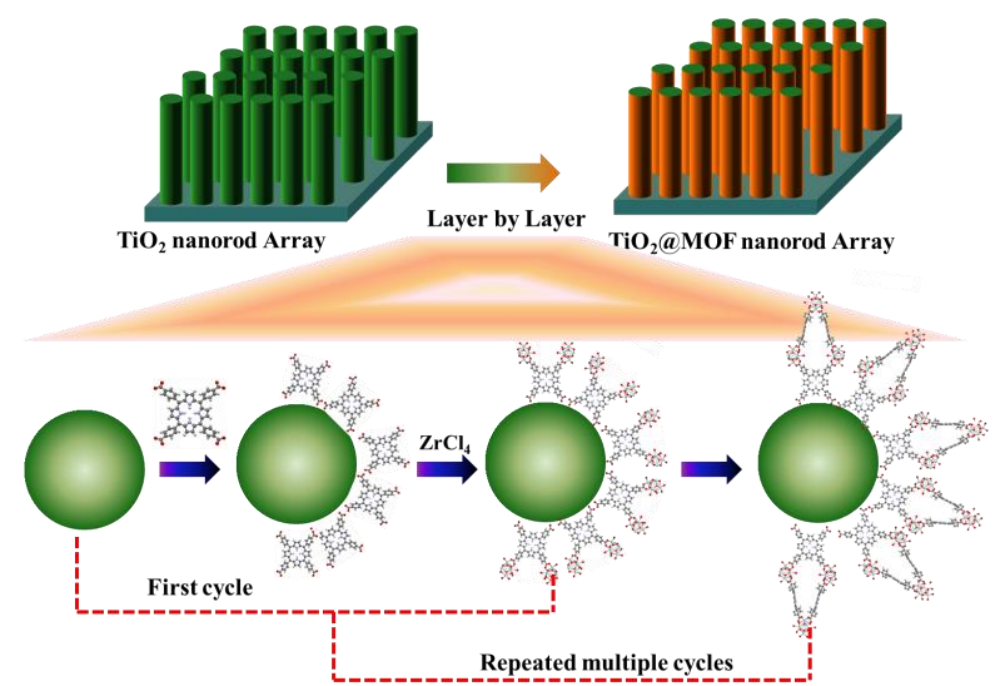

Figure 2. Scheme for the synthesis of $\mathrm{TiO}_{2} @ \mathrm{MOF}$ nanorod array photoanode through layer by layer method.

This $\mathrm{TiO}_{2} @ \mathrm{MOF}$ core-shell nanorod array photoanode, which is vertically aligned on a fluorine-doped tin oxide (FTO) electric contact, allowing for a long optical path-length along the longitudinal axis of the nanorods while maintaining a short path-length for hole transport to the photoanode/electrolyte interface along the radius of the nanorods (the transverse direction), as illustrated in Fig. 3.3. The p-type MOF coating provides a porous surface with more reaction active sites than rutile $\mathrm{TiO}_{2}$ alone, which is expected to improve the charge injection from the photoanode surface to the electrolyte. The formation of a $\mathrm{p}-\mathrm{n}$ heterojunction is expected to aid in charge 
extraction from the photoanode. Therefore, coating $\mathrm{TiO}_{2}$ with $\mathrm{MOF}$ is expected to improve overall photoelectrochemical water-splitting performance. In this study, surface photovoltage spectroscopy (SPV) and electrochemical analysis are performed to gain fundamental understanding of the roles of MOF in the $\mathrm{TiO}_{2} @ \mathrm{Co}-\mathrm{MOF}$ nanorod array photoanode.

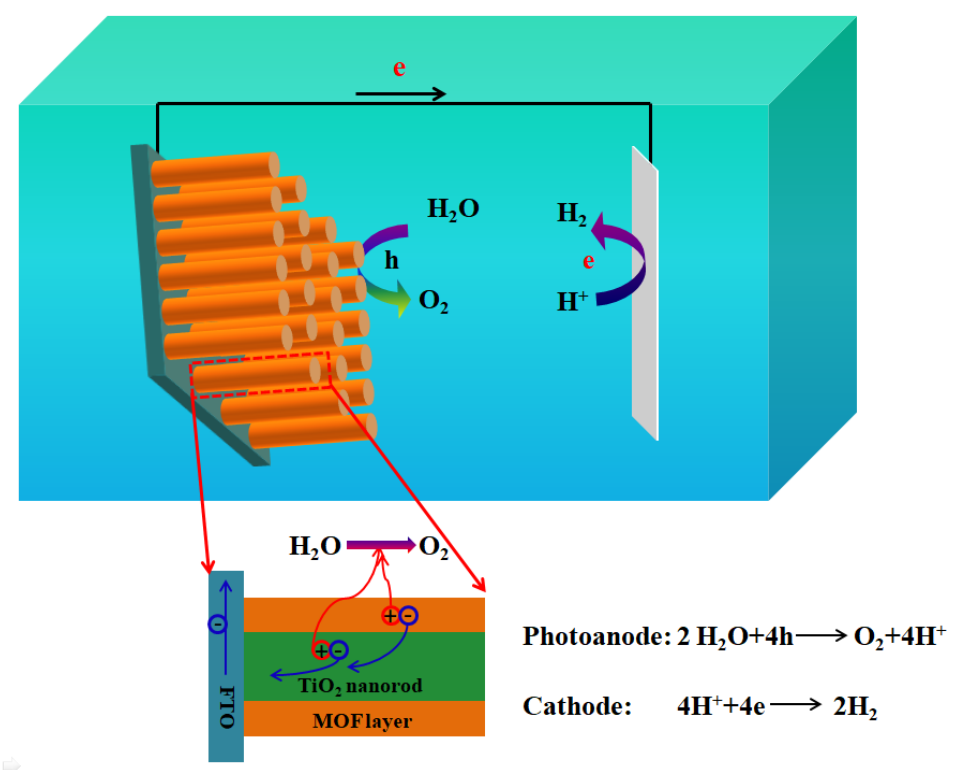

Figure 3.3: Photoelectrochemical water-splitting with $\mathrm{TiO}_{2} @ \mathrm{MOF}$ nanorod array photoanode.

\subsection{Results}

Figure 3.4 shows the single-crystalline rutile $\mathrm{TiO}_{2}$ nanorod array that was grown on the FTO substrate through a hydrothermal method. The SEM images reveal that the $\mathrm{TiO}_{2}$ nanorods were $\sim 2.2 \mu \mathrm{m}$ long with an edge length of $\sim 190 \mathrm{~nm}^{[28,29]}$. The scanning electron microscopy (SEM) (Fig. 3.4(c)) and transmission electron microscopy (TEM) images (Fig. 3.4(d)) display that there are MOF thin films covered on the surface of $\mathrm{TiO}_{2}$. Examination of individual nanorods through high-resolution TEM (HRTEM) images (Fig. 4(e)) shows that inner core $\mathrm{TiO}_{2}$ is completely crystalline along their entire lengths. Lattice fringes with interplanar spacing $d 110=$ $3.18 \mathrm{~A}$ are consistent with rutile phase of $\mathrm{TiO}_{2}$. 


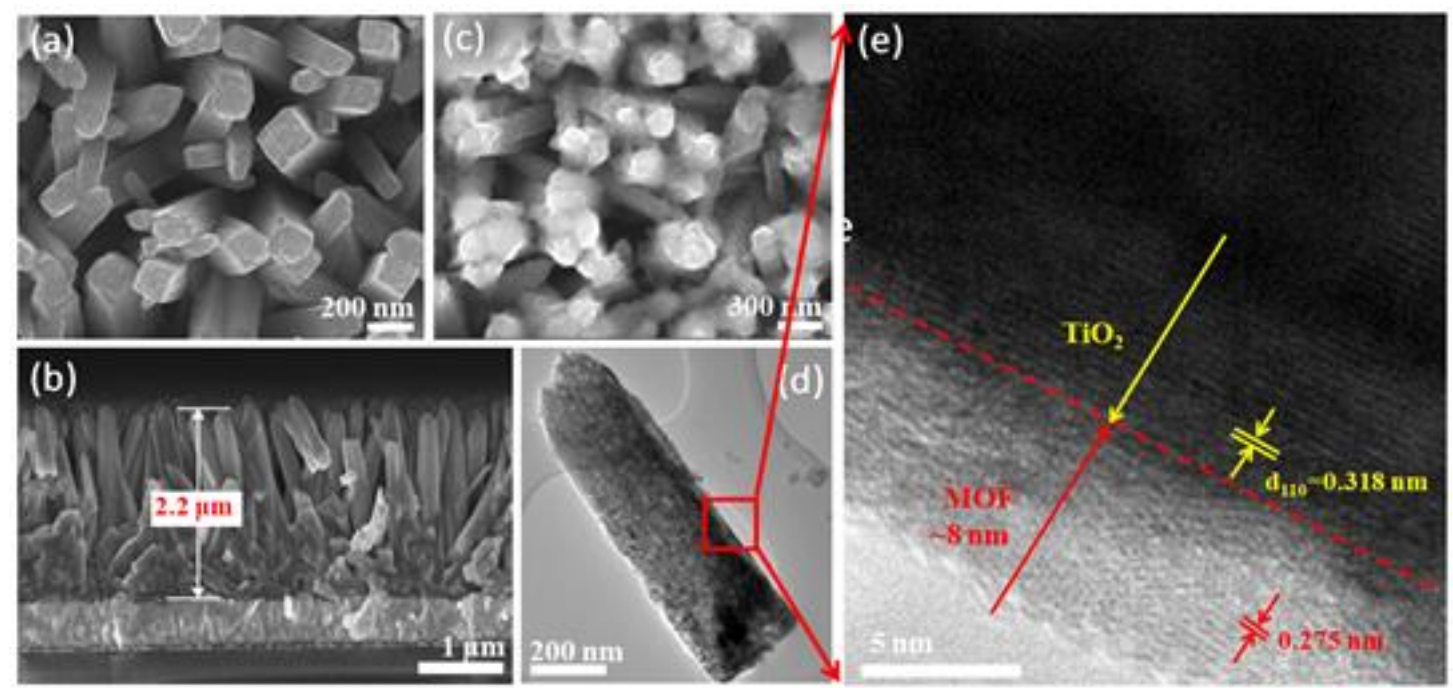

Figure 3.4: Microstructure of the $\mathrm{TiO}_{2} @ \mathrm{MOF}$ nanorod array. (a) Top-view SEM Images of $\mathrm{TiO}_{2}$ nanorod array; (b) Cross-section SEM Image of $\mathrm{TiO}_{2}$ NR array; (c) Top-view of $\mathrm{TiO}_{2} @ \mathrm{MOF}$ nanorod array; (d) TEM and (e) HRTEM images of a TiO $\mathrm{T}_{2} @ \mathrm{MOF}$ nanorod.

The optimized thickness of MOF layer was $\sim 8 \mathrm{~nm}$ with 5 repeated coating cycles based on the photocurrent density-voltage $(J-V)$ curves for $\mathrm{TiO}_{2} @ \mathrm{MOF}$ samples (Fig. 3.5). All subsequent data regarding the $\mathrm{TiO}_{2} @ \mathrm{MOF}$ samples refer to samples with the optimized 5 coating cycles for the rest of this manuscript unless otherwise denoted. The MOF structure has an interplanar spacings of $d 062=2.75 \mathrm{~A}$, coindicating its ordered structure. After the introducing of $\mathrm{Co}$ into the MOF, the morphology of the $\mathrm{TiO}_{2} @ \mathrm{Co}-\mathrm{MOF}$ doesn't change (Fig. 3.6). X-ray diffraction (XRD) was used to characterize the crystal structure of $\mathrm{TiO}_{2}$ nanoarray and MOF coating on $\mathrm{TiO}_{2}$ nanoarray. Fig. 3.7 displays the XRD pattern of the bare $\mathrm{TiO}_{2}$ nanoarray grown on the FTO substrate. All the diffraction peaks, which appear upon $\mathrm{TiO}_{2}$ nanorod growth, agree well with the tetragonal rutile phase. The $\mathrm{TiO}_{2} @ \mathrm{MOF}$ coating in Fig. 3.8 was prepared in a relatively large thickness (30 cycles of coating) to increase the XRD intensity. It can be seen that the XRD pattern matched well with the simulated pattern of PCN-225 ${ }^{[30]}$, which can be indexed as the (011), (013), (112) and (121) planes of PCN-225, demonstrating the presence of an ordered and preferentially oriented crystalline PCN-225 on the surface of $\mathrm{TiO}_{2}$. In addition, an XRD pattern was acquired from the powder sample of PCN-225, which indicated that the MOF coating exhibited the same crystal structure with the bulk counterpart. 


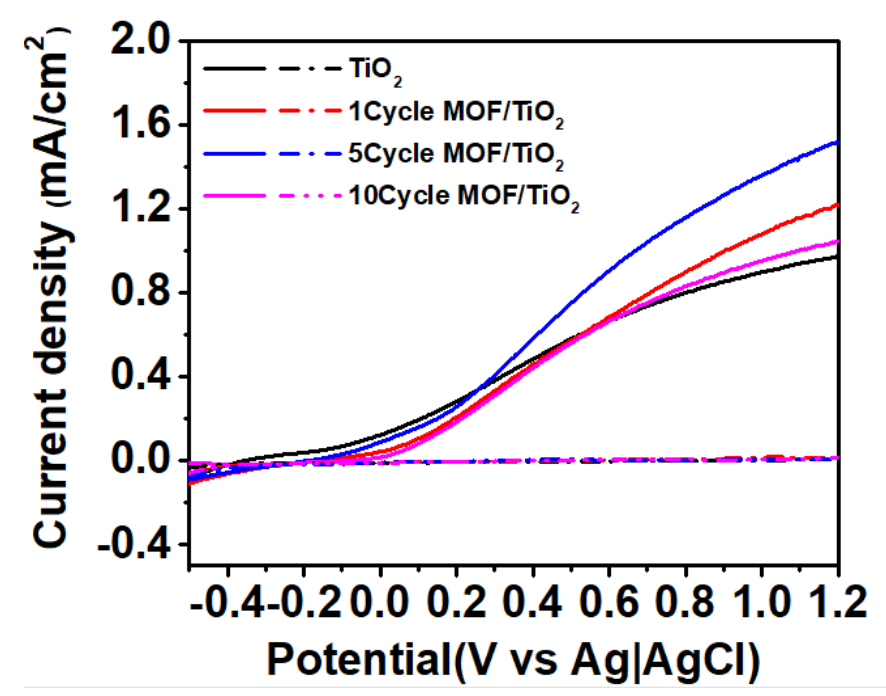

Figure 3.5: Photoelectrochemical performance of the $\mathrm{TiO}_{2}$ nanorod array and the $\mathrm{TiO}_{2} @ \mathrm{MOF}$ nanorod arrays with different numbers of MOF coating layers.

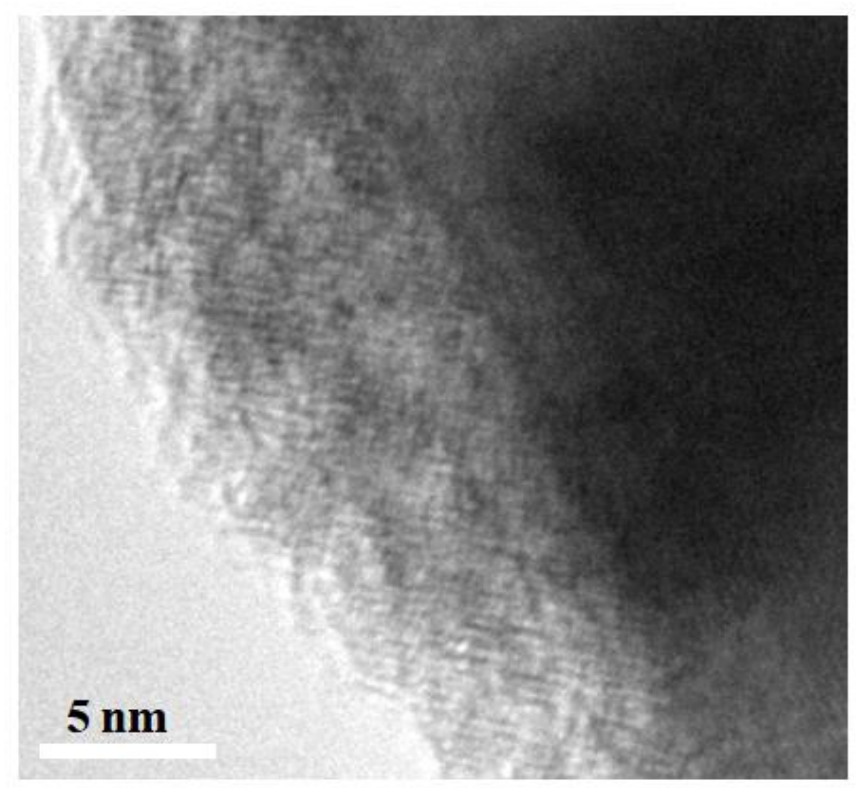

Figure 3.6: $\mathrm{HRTEM}$ image of a $\mathrm{TiO}_{2} @ \mathrm{Co}-\mathrm{MOF}$ nanorod. 


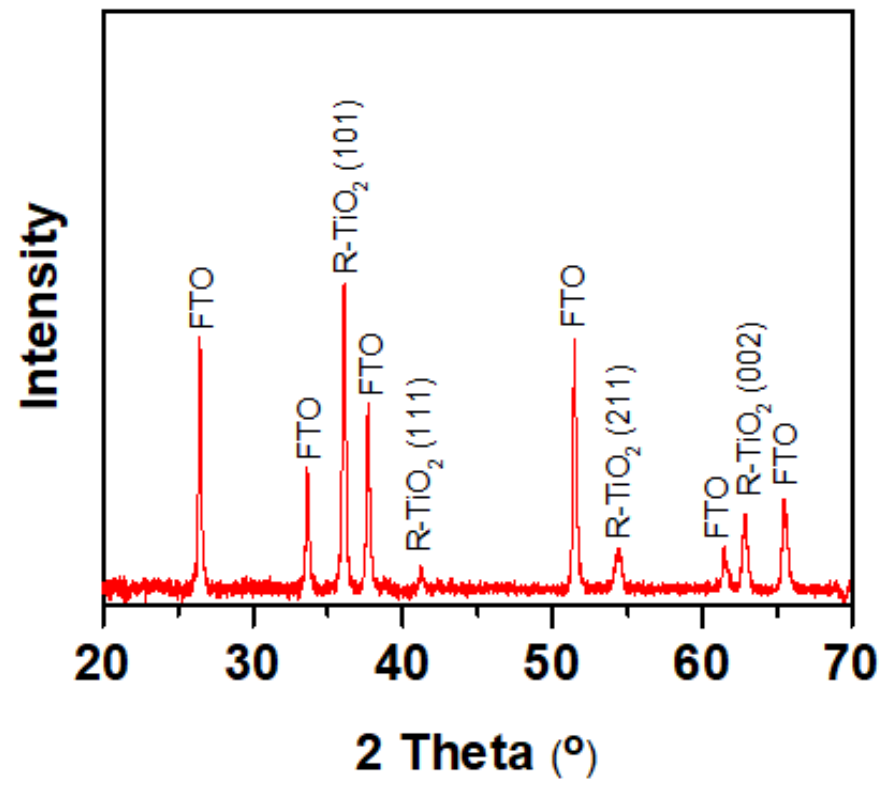

Figure 3.7: Powder X-ray diffraction profiles for the bare $\mathrm{TiO}_{2}$ nanorods on FTO substrate.

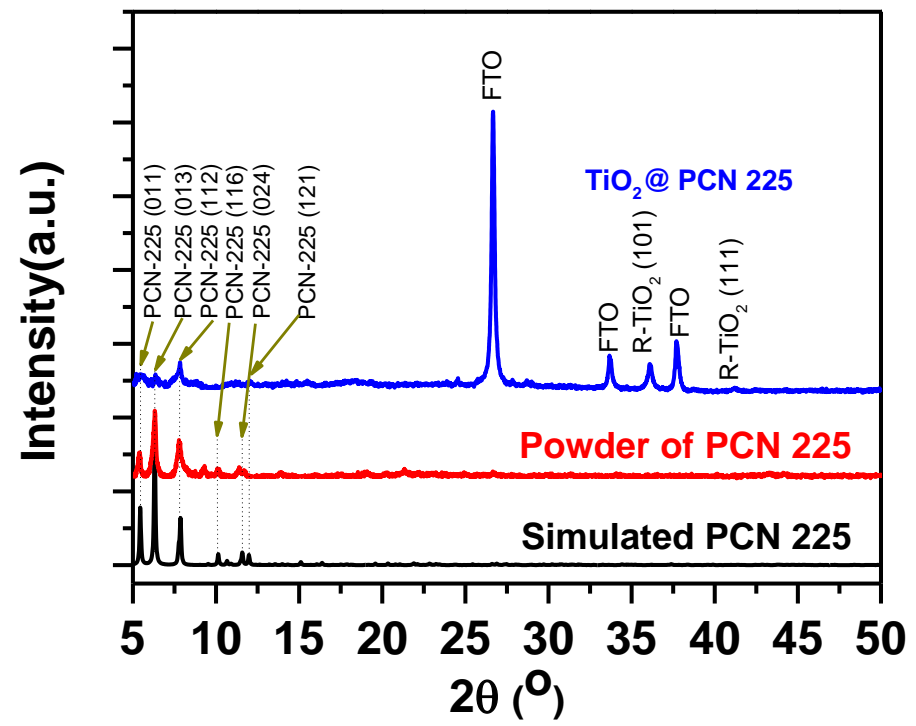

Figure 3.8: Powder $\mathrm{X}$-ray diffraction profiles for the $\mathrm{TiO}_{2} @ \mathrm{MOF}(\mathrm{PCN}-225)$, the $\mathrm{PCN}-225$ powder and the simulated PCN-225. The simulated PCN-225 was taken from the literature (Jiang, H-L., Feng, D. W., Wang, K. C., Gu, Z-Y., Wei, Z. W., Chen, Y-P., Zhou, H-C., J. Am. Chem. Soc. 2013, 135, 13934).

X-ray photoelectron spectroscopy (XPS) analysis was performed to confirm the growth and chemical composition of MOF structure. In the spectra of $\mathrm{TiO}_{2} @ \mathrm{MOF}$ nanorod array in Fig. 3.9 , the peaks corresponding to $\mathrm{C} 1 \mathrm{~s}(284.8 \mathrm{eV}), \mathrm{N} 1 \mathrm{~s}(398.2$ and $400.3 \mathrm{eV})$, 
$\mathrm{Zr} 3 \mathrm{~d}(182.5 \mathrm{eV})$ clearly indicate the presence of Zr-based porphyrin MOFs on the $\mathrm{TiO}_{2}$ nanorod array surface. The carbon and nitrogen elements were exclusively attributed to the porphyrin molecules while the zirconium element came from the metal clusters in the MOF. The $\mathrm{C} 1 \mathrm{~s}$ corelevel XPS spectrum can be deconvoluted into three subcomponents at 284.8, 286.6, and $288.8 \mathrm{eV}$ (Fig. 3.9(a) in the ESM), corresponding to the $\mathrm{C}-\mathrm{C}, \mathrm{C}-\mathrm{O}$ and $-\mathrm{COOH}$ bonds, respectively. The $\mathrm{N}$ 1s peak (Fig. 3.9(c)) was fitted to two subcomponents at $398.2 \mathrm{eV}(\mathrm{C}=\mathrm{N})$ and $400.3 \mathrm{eV}(\mathrm{C}-\mathrm{NH})^{[31]}$. A small signal corresponding to Ti $2 \mathrm{p}(458.7 \mathrm{eV})$ was detected, which indicated that the MOF coating was slightly thinner than the maximum escape depth for photoelectrons from the sample. This was consistent with the MOF layer thickness obtained from the HRTEM image in Fig. 3.4(e). After introducing of cobalt into the MOF coating to form the $\mathrm{TiO}_{2} @ \mathrm{Co}-\mathrm{MOF}$ nanorod array, a new peak corresponding to Co $2 \mathrm{p}$ was found at $781.7 \mathrm{eV}$ (Fig. 3.10(f)). Based on the $15.0 \mathrm{eV}$ doublet separation energy between Co $2 \mathrm{p} 3 / 2$ and Co $2 \mathrm{p} 1 / 2$, the introduced cobalt ions were determined to be in the $\mathrm{Co}^{3+}$ state. In addition, a $0.2 \mathrm{eV}$ shift in binding energy for both $\mathrm{N}$ subpeaks indicated that the $\mathrm{Co}$ (III) was coordinated within the center of the porphyrin ligands ${ }^{[32]}$. FTIR spectra in Fig. 3.11 show significant difference between the $\mathrm{TiO}_{2} @-\mathrm{MOF}$ and the $\mathrm{TiO}_{2} @ \mathrm{Co}-$ MOF samples. The N-H bond stretching and bending frequencies of TCPP located at $994 \mathrm{~cm}^{-1}$ decreased dramatically after cobalt ions were incorporated with porphyrin, which indicates the formation of a cobalt porphyrin compound. No changes were observed for the peaks at $1656 \mathrm{~cm}^{-1}$ $(\mathrm{C}=\mathrm{N}), 1287 \mathrm{~cm}^{-1}(\mathrm{C}-\mathrm{O}), 857 \mathrm{~cm}^{-1}(\mathrm{C}-\mathrm{H}$ bending $)$ attributed to the porphyrin molecules ${ }^{[33,34]}$

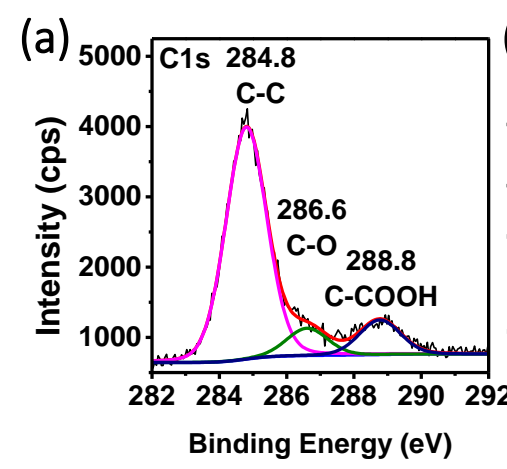

(d)

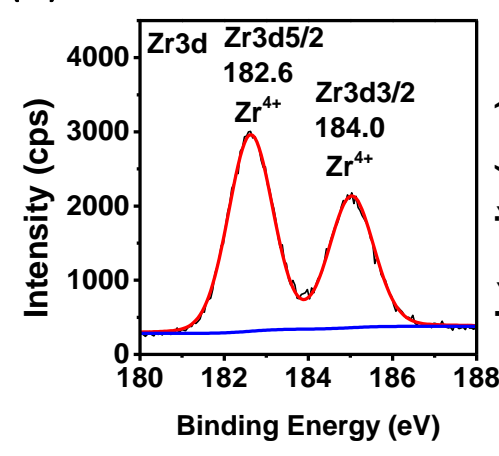

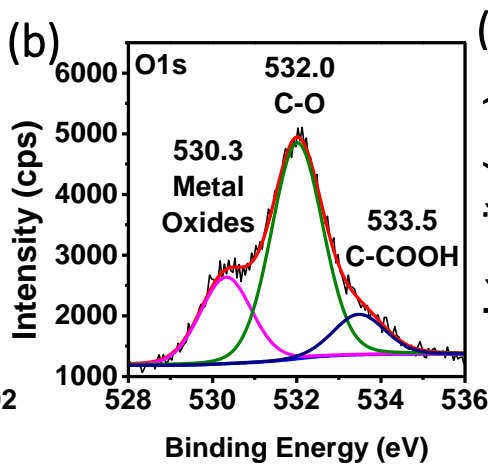

(e)

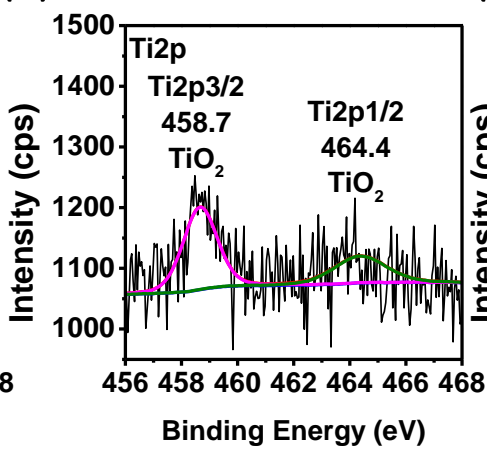

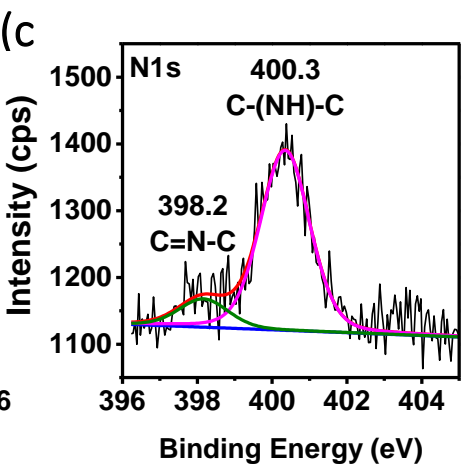

(f)

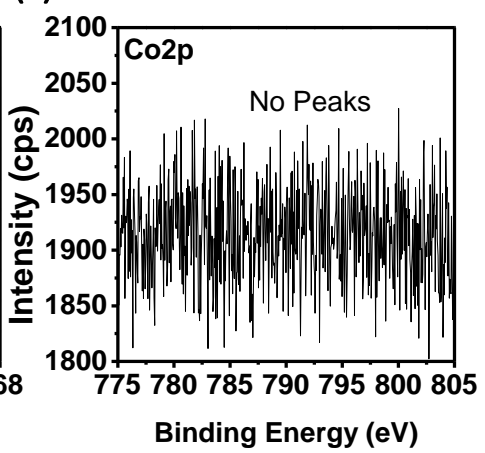

Figure 3.9: XPS Spectra for $\mathrm{TiO}_{2} @ M O F$ samples; (a) C1s, (b) O1s, (c) N1s, (d) Zr3d, (e) Ti2p, (f) $\mathrm{Co} 2 \mathrm{p}$. 

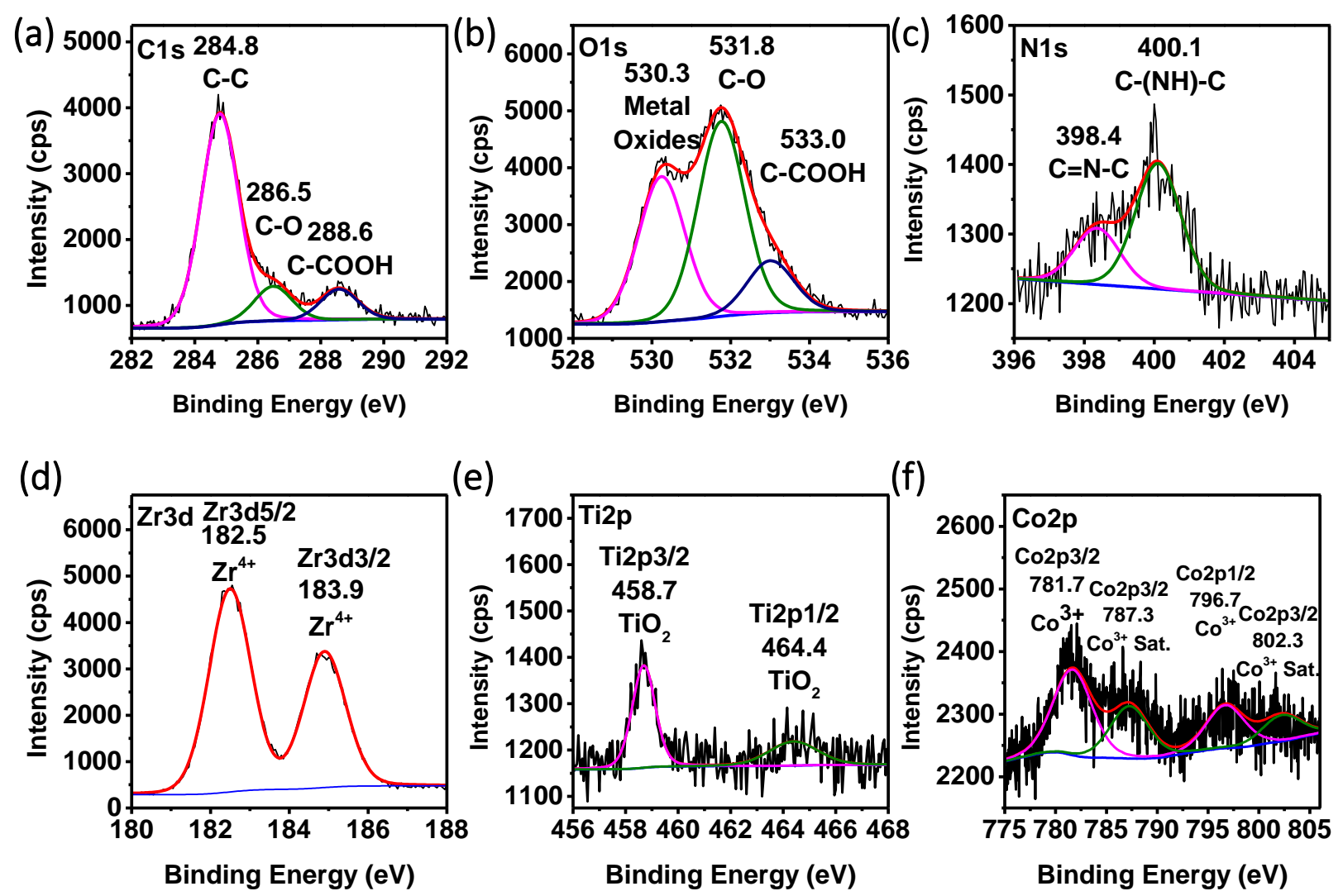

Figure 3.10: XPS Spectra for $\mathrm{TiO}_{2} @$ Co-MOF samples; (a) C1s, (b) O1s, (c) N1s, (d) Zr3d, (e) Ti2p, (f) Co2p.

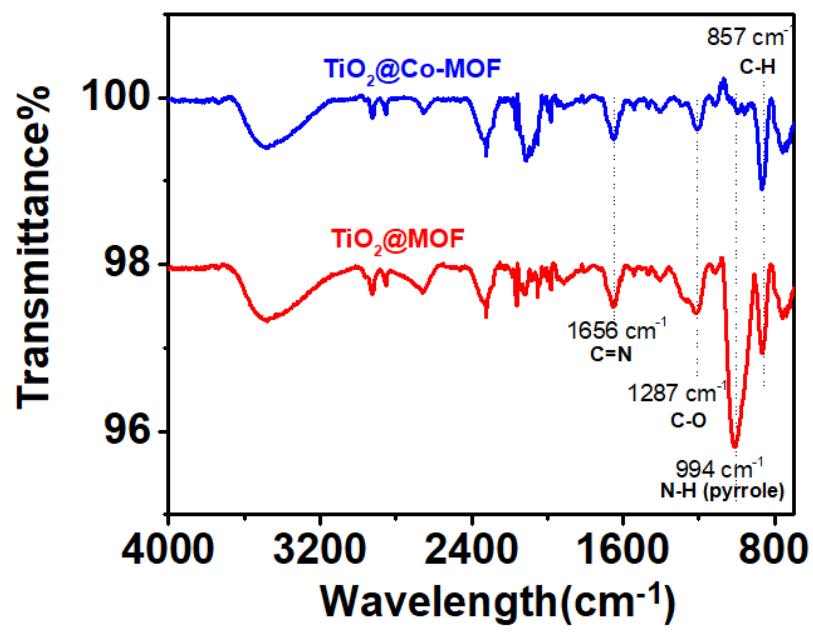

Figure 3.11: FTIR Spectra for $\mathrm{TiO}_{2} @ \mathrm{MOF}, \mathrm{TiO}_{2} @ \mathrm{Co}-\mathrm{MOF}$ samples.

Photoelectrochemical testing was performed on the $\mathrm{TiO}_{2}$ and $\mathrm{TiO}_{2} @ \mathrm{MOF}$ samples (Fig. 3.12(a)). Dark currents of the $\mathrm{TiO}_{2}, \mathrm{TiO}_{2} @ \mathrm{MOF}, \mathrm{TiO}_{2} @ \mathrm{Co}-\mathrm{MOF}$ samples indicate that the water oxidation reaction only occurred under light illumination. The $J-V$ curves show that there was 
significant enhancement in photocurrent density after $\mathrm{MOF}$ was coated onto the $\mathrm{TiO}_{2}$ nanorod array. The bare $\mathrm{TiO}_{2}$ nanorod array displayed a photocurrent density of $1.09 \mathrm{~mA} / \mathrm{cm}^{2}$ at $1.23 \mathrm{~V}$ (vs. RHE). The photocurrent density of the $\mathrm{TiO}_{2} @ \mathrm{MOF}$ core-shell nanorod array increased to 1.99 $\mathrm{mA} / \mathrm{cm}^{2}$ at $1.23 \mathrm{~V}$, which was 1.8 times greater than that of the $\mathrm{TiO}_{2}$ nanorod array. After $\mathrm{Co}^{3+}$ was introduced into the porphyrin, the photocurrent density of $\mathrm{TiO}_{2} @ \mathrm{Co}-\mathrm{MOF}$ sample exhibited $2.93 \mathrm{~mA} / \mathrm{cm}^{2}$ at $1.23 \mathrm{~V}$, which was approximately 2.7 times greater than that of the $\mathrm{TiO}_{2}$ nanorod array alone. It is worth noting that the onset potentials for all the three samples were the same (Fig. 3.12(a)).
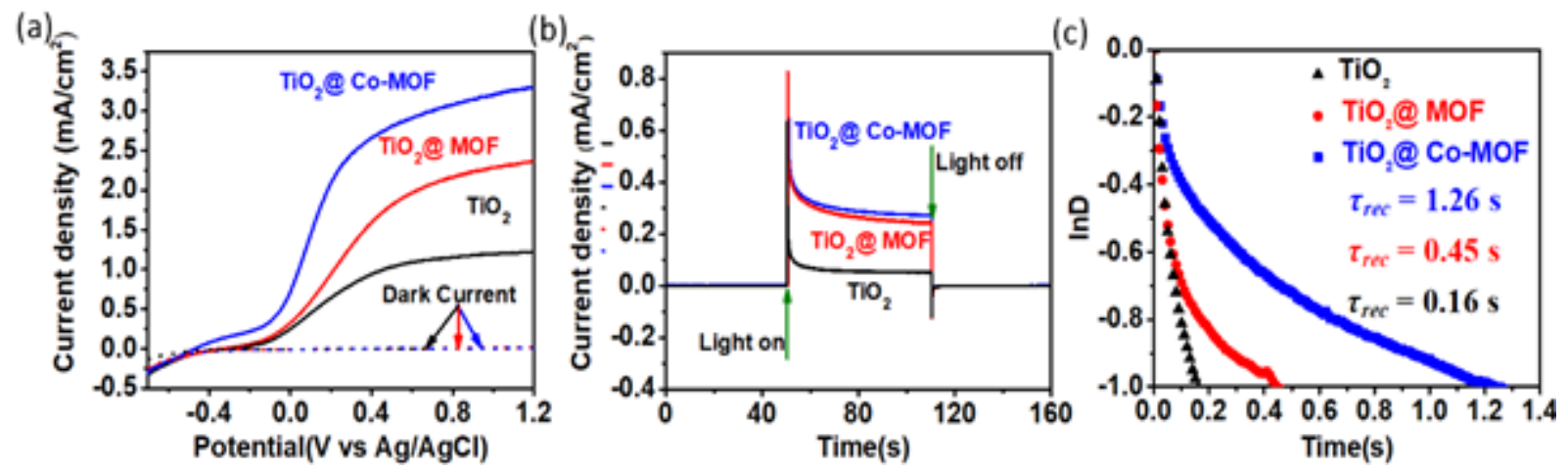

Figure 3.12: Photoelectrochemical performance of nanorod array photoanodes in $0.2 \mathrm{M} \mathrm{Na}_{2} \mathrm{SO}_{4}$ (pH=7). (a) Photocurrent-applied potential (J-V) curves for $\mathrm{TiO}_{2}, \mathrm{TiO}_{2} @ \mathrm{MOF}$ and $\mathrm{TiO}_{2} @ \mathrm{Co}-$ MOF nanorod arrays under irradiation of the full-spectrum unfiltered Xe lamp light; (b) Chronoamperometry $\left(J\right.$ - $t$ ) curves at $0 \mathrm{~V}$ (vs. $\mathrm{Ag} \mid \mathrm{AgCl}$ ); (c) $\ln (D)$ - $t$ plots for $\mathrm{TiO}_{2}, \mathrm{TiO}_{2} @ \mathrm{MOF}$ and $\mathrm{TiO}_{2} @ \mathrm{Co}-\mathrm{MOF}$ nanorod arrays.

Mott-Schottky (M-S) experiments were performed to clarify the nature of the junction formed between $\mathrm{TiO}_{2}$ and MOF. The negative linear slope on the M-S plot for the porphyrin MOF film on FTO glass (Fig. 3.13(a)) indicates that the porphyrin MOF alone showed a p-type semiconducting behavior. The p-type nature of this MOF was confirmed by the negative in-phase and out-of-phase surface photovoltage (SPV) signals for the MOF alone (Fig. 3.14). The positive linear slope on the M-S plot proved the n-type nature of the $\mathrm{TiO}_{2}$ nanorod array (Fig. 3.13(b)). The "inverted V" M-S curves suggested the formation of the $\mathrm{p}-\mathrm{n}$ junction between $\mathrm{MOF}$ and $\mathrm{TiO}_{2}$ in the $\mathrm{TiO}_{2} @ \mathrm{MOF}$ and $\mathrm{TiO}_{2} @ \mathrm{Co}-\mathrm{MOF}$ samples (Figs. 3.13(c) and $3.13(\mathrm{~d}))^{[35]}$. Given the thin nature $(\sim 8 \mathrm{~nm})$ of the MOF coating on the $\mathrm{TiO}_{2}$ nanorods, the p-type MOF layer is likely fully-depleted. There is insufficient thickness for a barrier to hole-injection to electrolyte to form while the built-in electric field can still form between the $\mathrm{TiO}_{2}$ core and MOF shell, which will help drive the photogenerated holes from $\mathrm{TiO}_{2}$ into the MOF layer. In addition, there was no evident difference in the flat-band potential among the $\mathrm{TiO}_{2}, \mathrm{TiO}_{2} @ \mathrm{MOF}$, and $\mathrm{TiO}_{2} @ \mathrm{Co}-\mathrm{MOF}$ nanorod arrays according to the M-S plots. 

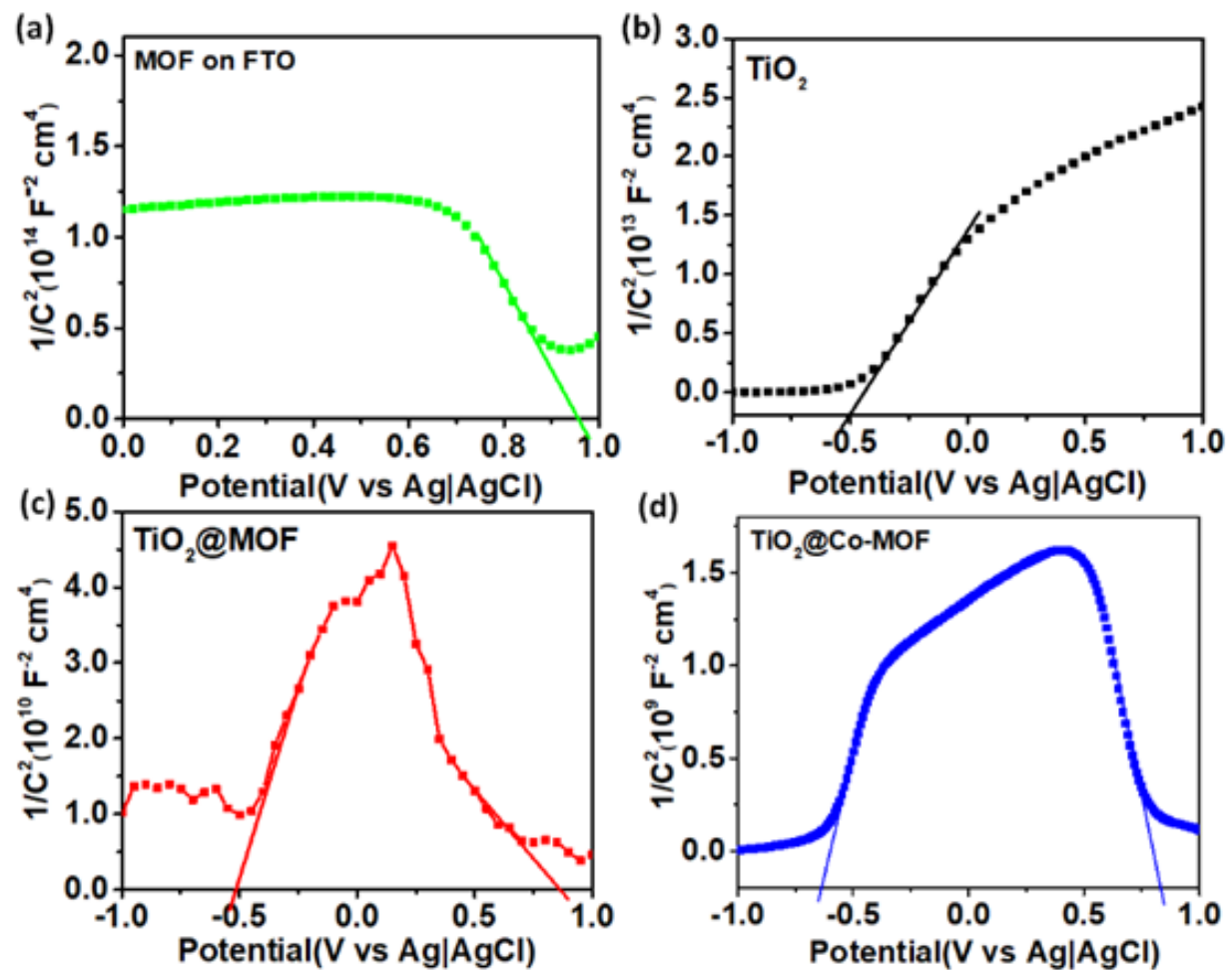

Figure 3.13: Mott-Schottky (M-S) plot measurement. (a) MOF film on FTO glass; (b) $\mathrm{TiO}_{2}$ nanorod array; (c) $\mathrm{TiO}_{2} @ \mathrm{MOF}$; and (d) $\mathrm{TiO}_{2} @ \mathrm{Co}-\mathrm{MOF}$ nanorod array.
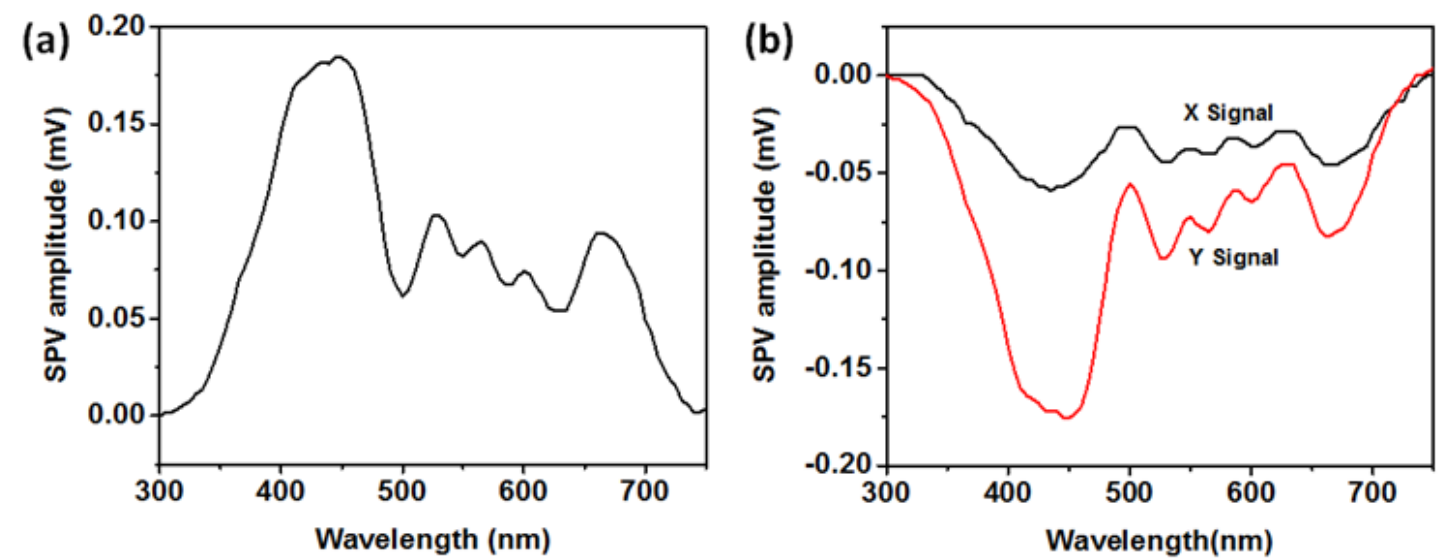

Figure 3.14: (a) Surface photovoltage (SPV) amplitude and (b) in-phase (X) and out-of-phase (Y) components of the SPV signal for MOF film on FTO glass.

To understand the roles of the MOF coating with and without $\mathrm{Co}$ (III) in enhancing the overall photocurrent density of PEC, the transient photocurrent ( $J-t$ curves) was measured at $0 \mathrm{~V}$ (vs. $\mathrm{Ag} / \mathrm{AgCl}, 0.61 \mathrm{~V}$ vs. RHE) to investigate the charge recombination behavior at a potential where photocurrent onset has just started for all samples. The $J-t$ curves in Fig. 3.12(b) were used to construct the plots of the natural logarithm of dimensionless parameter $(D)$ to determine the apparent charge carrier lifetime $\left(\tau_{\text {rec }}\right)$ in each sample (see Equation 3 in Section 3.4.6.2 for 
calculation). $\tau_{\text {rec }}$ was $0.16 \mathrm{~s}$ for the $\mathrm{TiO}_{2}$ nanorod array alone. $\tau_{\text {rec }}$ increased to $0.29 \mathrm{~s}$ after coating MOF onto $\mathrm{TiO}_{2}$, and further increased to $1.26 \mathrm{~s}$ after $\mathrm{Co}^{3+}$ was introduced into the MOF (Fig. 3.12(c)). An increase in $\tau_{\text {rec }}$ indicated that coating of MOF or Co-MOF on the $\mathrm{TiO}_{2}$ nanorod array facilitated the charge extraction inside the photoanode and the charge injection from the photoanode to the electrolyte, which in turn resulted in significant enhancement in photocurrent density.

To determine the relative enhancement of the charge injection and the charge separation by the MOF and Co-MOF coatings on the $\mathrm{TiO}_{2}$ nanorod array, $J-V$ curves were acquired in the $\mathrm{Na}_{2} \mathrm{SO}_{4}$ aqueous solutions in the presence and absence of triethanolamine (TEOA) under illumination of a $300 \mathrm{~W}$ Xe lamp (Fig. 3.15). The incident light was unfiltered for measuring charge injection efficiencies and filtered using a $275-375 \mathrm{~nm}$ bandpass filter for measuring relative charge separation efficiency enhancement. The total steady-state water oxidation photocurrent density $\left(J_{\text {photocurrent/H2O }}\right)$ generated in the aqueous electrolyte is expressed as: ${ }^{[36]}$

$$
J_{\text {photocurrent/H2O }}=J_{\text {absorb }} \times P_{\text {sep }} \times P_{\text {inject }},
$$

where $J_{\text {absorb }}$ is the theoretical maximum photocurrent determined by the optical band gap and optical absorption coefficient of the photoanode; $P_{\text {sep }}$ is the charge separation efficiency that reflects the fraction of the photogenerated holes reaching the photoanode/ electrolyte interface; and $P_{\text {inject }}$ is the charge injection efficiency that reflects the fraction of the photogenerated holes injected to the redox pair in the electrolyte. If $10 \mathrm{vol} . \%$ TEOA is present in the electrolyte as a hole scavenger, $P_{\text {inject }}$ can be considered as approximately $100 \%$ due to the fast oxidation kinetics of TEOA $^{[36,37]}$. Based on this, $P_{\text {inject }}$ can be calculated by taking the ratio of the photocurrent densities of the photoanode in electrolyte without and with TEOA. $P_{\text {inject }}$ was estimated to be $58 \%, 71 \%$ and $88 \%$ at $1.23 \mathrm{~V}$ (vs. RHE) for the $\mathrm{TiO}_{2}, \mathrm{TiO}_{2} @ \mathrm{MOF}$ and $\mathrm{TiO}_{2} @ \mathrm{Co}-\mathrm{MOF}$ photoanodes, respectively. The low charge injection efficiency indicated the abundant surface trap-states on bare $\mathrm{TiO}_{2}$. Coating $\mathrm{TiO}_{2}$ with MOF and Co-MOF passivated the surface trap-states, and the porous MOF favored the charge injection into the electrolyte. To determine the relative change in charge separation efficiency, the photocurrent density was measured in the TEOA-containing electrolyte under $275-375 \mathrm{~nm}$ bandpass filtered illumination to minimize the differences in $J_{\text {absorb }}$ between sample types. 

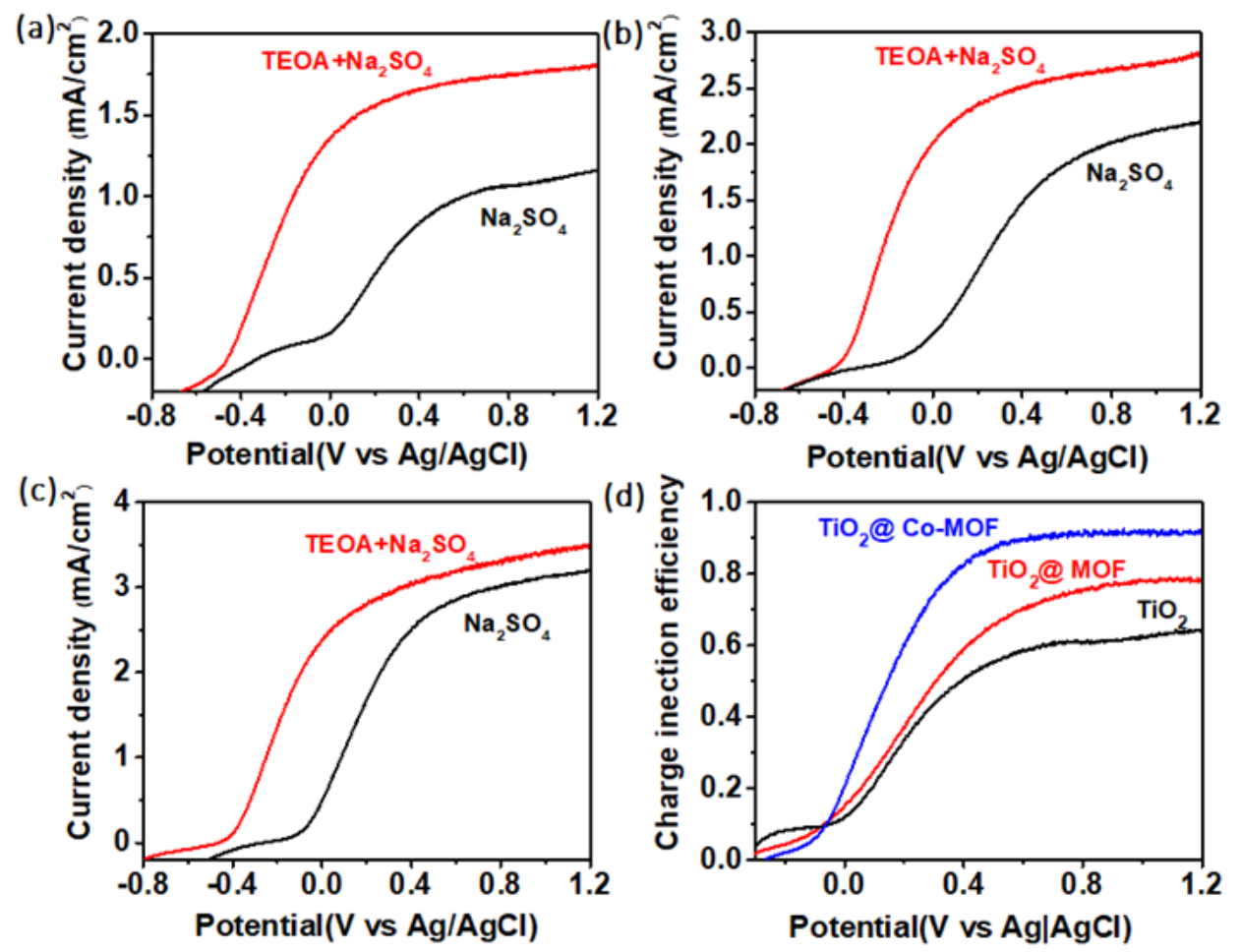

Figure 3.15: Charge injection efficiency for the PECs with different photoanodes in the $\mathrm{Na}_{2} \mathrm{SO}_{4}$ solution electrolyte in the presence and absence of TEOA. (a) J-V curves obtained from the PEC with the $\mathrm{TiO}_{2}$ photoanode; (b) J-V curves obtained from the PEC with the $\mathrm{TiO}_{2} @ \mathrm{MOF}$ photoanode; (c) J-V curves obtained from the PEC with the $\mathrm{TiO}_{2} @ \mathrm{Co}-\mathrm{MOF}$ photoanode; (d) charge injection efficiency for $\mathrm{TiO}_{2}, \mathrm{TiO}_{2} @ \mathrm{MOF}, \mathrm{TiO}_{2} @ \mathrm{Co}-\mathrm{MOF}$ photoanodes in the $\mathrm{Na}_{2} \mathrm{SO}_{4}$ solution electrolyte.

The relative enhancement in charge separation efficiency can be found by taking the ratio of the photocurrent density in the TEOA-containing electrolyte for the $\mathrm{TiO}_{2} @ \mathrm{MOF}$ or $\mathrm{TiO}_{2} @ \mathrm{Co}-$ MOF with respect to the bare $\mathrm{TiO}_{2}$ nanorod array. Figure 3.16 reveals the resulting $J-V$ curves in TEOA electrolyte and the relative charge separation efficiency enhancement by the MOF and CoMOF. The $\mathrm{TiO}_{2} @$ Co-MOF sample provided a charge separation efficiency of 2.7 times larger than the bare $\mathrm{TiO}_{2}$ photoanode. In short, the overall photocurrent enhancement in the $\mathrm{TiO}_{2} @ \mathrm{MOF}$ or $\mathrm{TiO}_{2} @ \mathrm{Co}-\mathrm{MOF}$ samples were ascribed to the relative changes in both the charge separation and the charge injection efficiencies. 
(a)

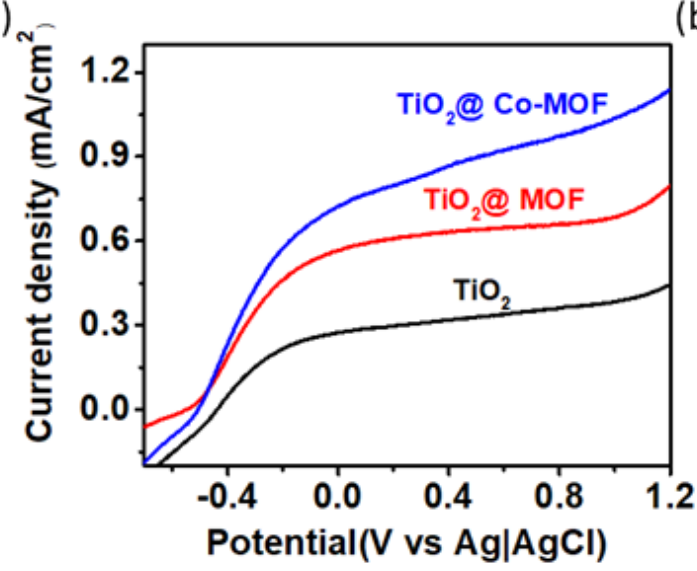

(b)

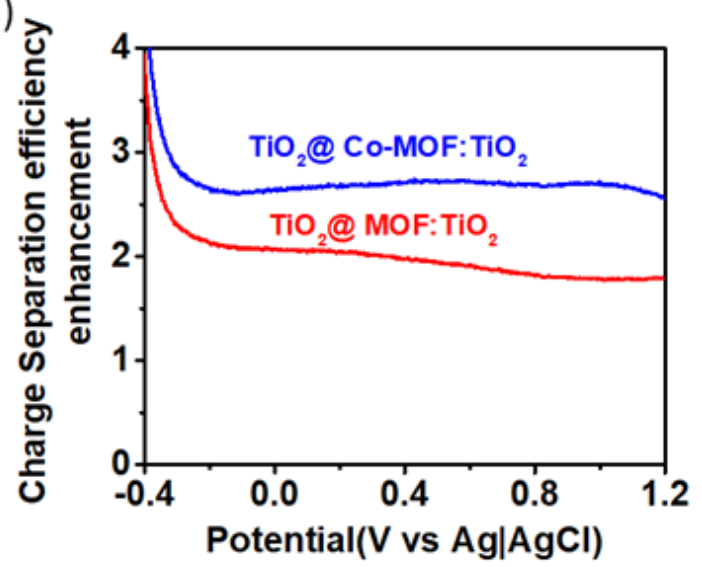

Figure 3.16. Charge separation efficiency enhancement in the photoanodes under incident light at the window of 275-375 nm. (a) J-V curves obtained from the PEC with the $\mathrm{TiO}_{2}, \mathrm{TiO}_{2} @ \mathrm{MOF}$, $\mathrm{TiO}_{2} @ \mathrm{Co}-\mathrm{MOF}$ photoanodes in the TEOA-containing $\mathrm{Na}_{2} \mathrm{SO}_{4}$ solution electrolyte; (b) enhancement factors for the charge separation.

The incident photon-to-electron conversion efficiency (IPCE) spectrum was measured to correlate the quantum efficiency with the wavelength of the incident light (Fig. 3.17). Figure 3.17(a) displays the ultraviolet-visible (UV-Vis) spectra of the three photoanodes. A significant increase in IPCE was observed in the $\mathrm{TiO}_{2} @ \mathrm{MOF}$ and $\mathrm{TiO}_{2} @ \mathrm{Co}-\mathrm{MOF}$ samples with reference to the bare $\mathrm{TiO}_{2}$ nanorod array (Fig. 3.17(b)). In the wavelength range of 320-420 nm, the IPCE enhancement factor was obtained by the IPCE ratio of $\mathrm{TiO}_{2} @ \mathrm{MOF}$ (or $\mathrm{TiO}_{2} @ \mathrm{Co}-\mathrm{MOF}$ ) to $\mathrm{TiO}_{2}$; and its value was 10.8 at $350 \mathrm{~nm}$ for the $\mathrm{TiO}_{2} @ \mathrm{Co}-\mathrm{MOF}$, and decreased to a nearly constant of 1.8 in the range of $420-580 \mathrm{~nm}$ (Fig. 3.17(d)). Close examination of the IPCE at $\lambda \geq 420 \mathrm{~nm}$ (Fig. 3.17(c)), where the $\mathrm{TiO}_{2}$ nanorod array had negligible light absorption, the $\mathrm{TiO}_{2} @ \mathrm{MOF}$ and $\mathrm{TiO}_{2} @$ Co-MOF showed only a small IPCE ( $\leq 0.06 \%$ at wavelengths between 460 and $580 \mathrm{~nm}$ ). The IPCE was very small for both the $\mathrm{TiO}_{2} @ \mathrm{MOF}$ and $\mathrm{TiO}_{2} @ \mathrm{Co}-\mathrm{MOF}$ samples in this spectral range. Although coating $\mathrm{MOF}$ and $\mathrm{Co}-\mathrm{MOF}$ onto $\mathrm{TiO}_{2}$ extended the light absorption spectral range up to $700 \mathrm{~nm}$ (Fig. 3.17(a)), the extension of light absorption range by the MOF coating was not responsible for the overall photocurrent enhancement in the PECs with the $\mathrm{TiO}_{2} @ \mathrm{MOF}$ and $\mathrm{TiO}_{2} @ \mathrm{Co}-\mathrm{MOF}$ photoanodes. In other words, light absorption by the MOF had negligible contribution to photo-generation of electrons and holes in the MOF layer. This was not surprising because the MOF layer was only $8 \mathrm{~nm}$ thick, which led to a limited optical path length in the MOF layer. Instead, the photocurrent enhancement by the $\mathrm{MOF}$ coating onto $\mathrm{TiO}_{2}$ was mainly attributed to the IPCE increase in the spectra range below $420 \mathrm{~nm}$, which came from an improvement in the charge separation efficiency and charge injection efficiency. The applied bias photon-to-current efficiency $(\mathrm{ABPE})^{[38]}$ was shown in Fig. 3.18. 

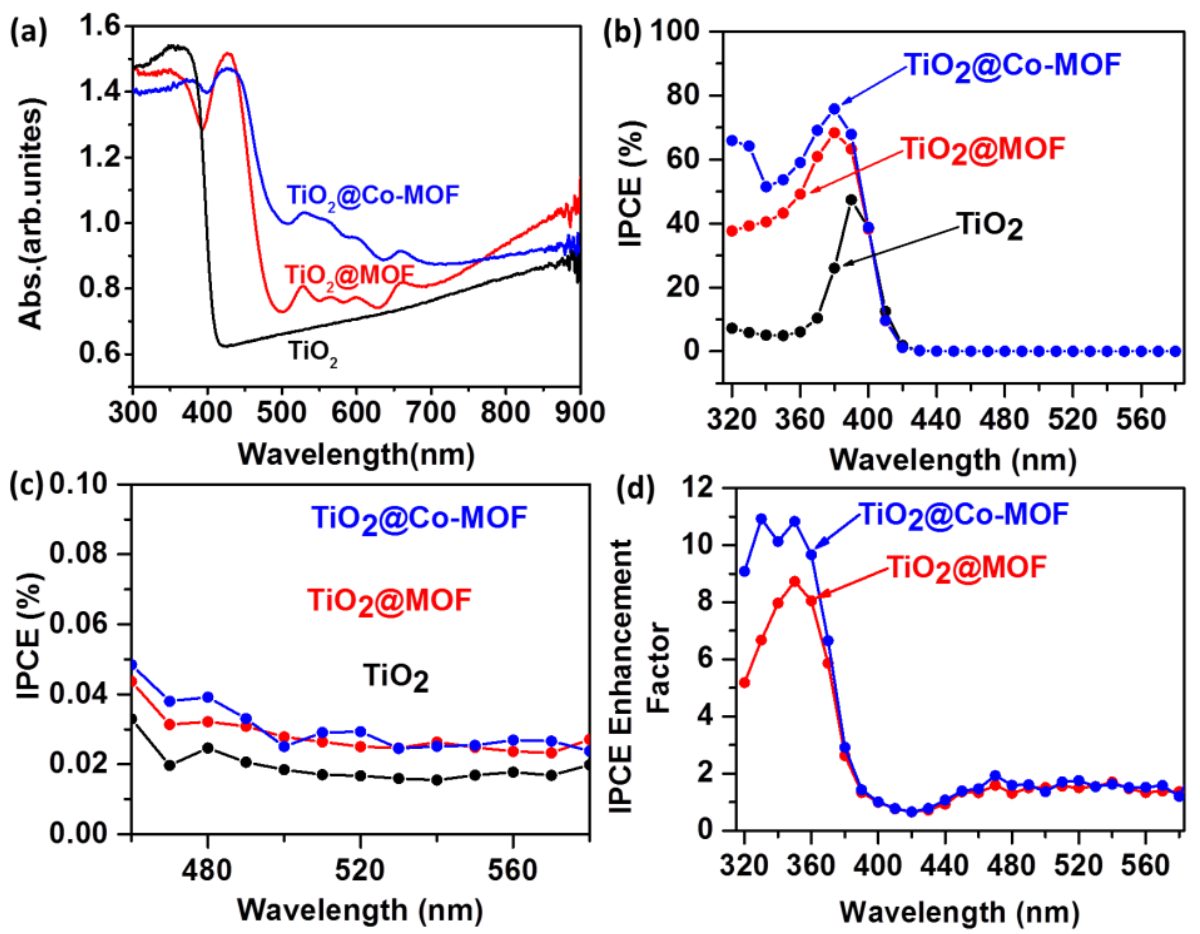

Figure 3.17: Optical absorption and IPCE measurement. (a) UV-Vis absorbance spectra; (b) IPCE spectra for $\mathrm{TiO}_{2}, \mathrm{TiO}_{2} @ \mathrm{MOF}$, and $\mathrm{TiO}_{2} @ \mathrm{Co}-\mathrm{MOF}$ nanorod arrays; (c) Magnified IPCE spectra between $460 \mathrm{~nm}$ and $580 \mathrm{~nm}$ for all samples; (d) IPCE enhancement for the $\mathrm{TiO}_{2} @ \mathrm{MOF}$, and $\mathrm{TiO}_{2} @ \mathrm{Co}-\mathrm{MOF}$ nanorod array.

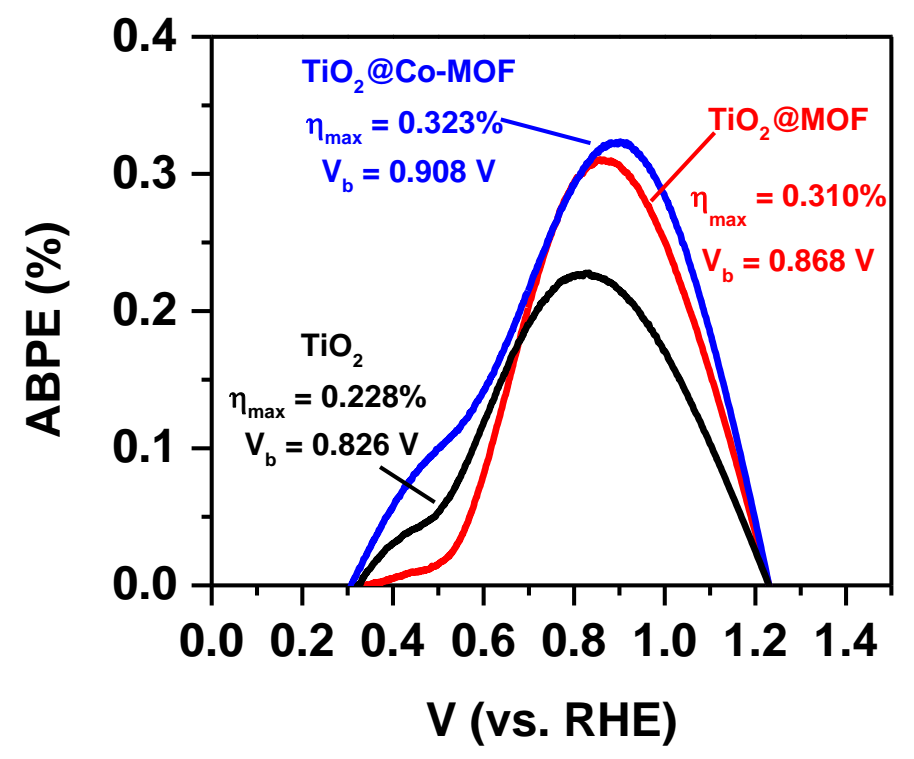

Figure 3.18: The applied bias photon-to-current efficiency (ABPE) of $\mathrm{TiO}_{2}, \mathrm{TiO}_{2} @ \mathrm{MOF}$, and $\mathrm{TiO}_{2} @ \mathrm{Co}-\mathrm{MOF}$ samples. 
It is important to reiterate that an unfiltered $300 \mathrm{~W}$ Xe lamp with an output power density set to $100 \mathrm{~mW} / \mathrm{cm}^{2}$ was used as the illumination source for all measured $J-V$ and $J-t$ curves. The resulting power of incident light exceeded the normal incident illumination power of the solar AM1.5 spectrum. As such, the photocurrent density obtained for the $\mathrm{TiO}_{2} @ \mathrm{MOF}$ and $\mathrm{TiO}_{2} @ \mathrm{Co}-$ MOF samples in Fig. 3.12(b) exceeded the theoretical maximum achievable photocurrent density for rutile $\mathrm{TiO}_{2}$ under AM1.5 illumination is $1.8 \mathrm{~mA} / \mathrm{cm}^{2}{ }^{[39]}$. The $J-V$ curves under irradiation of the simulated AM 1.5G light source are provided for better comparation with the literature (Fig. 3.19). The bare $\mathrm{TiO}_{2}$ nanorod array displayed a photocurrent density of $0.84 \mathrm{~mA} / \mathrm{cm}^{2}$ at $1.23 \mathrm{~V}$ (vs. RHE), which was comparable with the data in Refs. ${ }^{[40,41]}$.

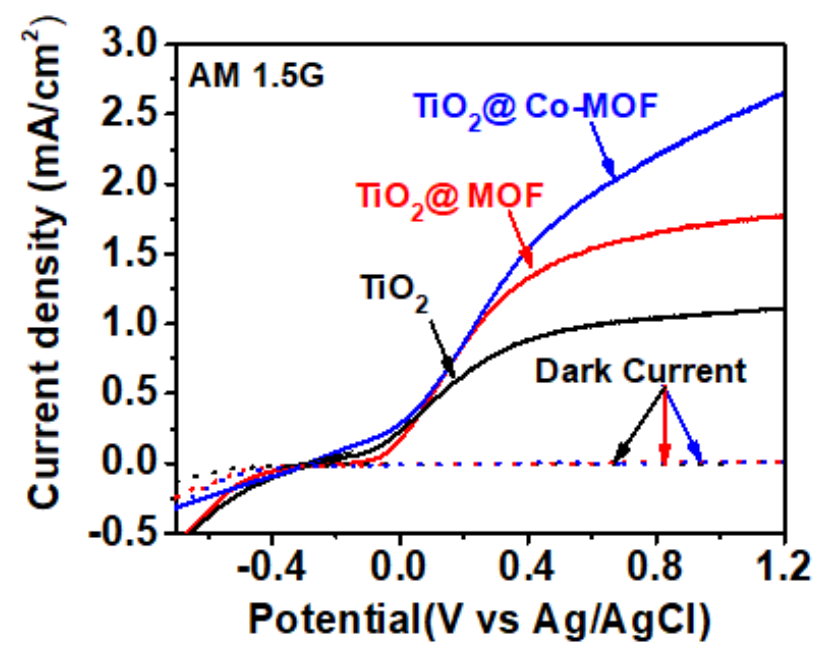

Figure 3.19: Photoelectrochemical performance of the nanorod array photoanodes. (a) Photocurrent-applied potential (J-V) curves for the $\mathrm{TiO}_{2}, \mathrm{TiO}_{2} @ \mathrm{MOF}$ and $\mathrm{TiO}_{2} @ \mathrm{Co}-\mathrm{MOF}$ nanorod arrays under irradiation of the simulated AM 1.5G light source.

Surface photovoltage spectroscopy is a nondestructive surface characterizing technique by monitoring change in the surface potential developed under light illumination ${ }^{[42-44]}$. In this chapter, surface photovoltage spectroscopy is used to determine the magnitude of photogenerated surface charge in the $\mathrm{TiO}_{2}$ and $\mathrm{TiO}_{2} @ \mathrm{MOF}$ samples as a function of the wavelength of incident light. It can be seen from Fig. 3.20(a) that the $\mathrm{TiO}_{2}$ nanorod array exhibited a small, positive in-phase $(X)$ surface photovoltage signal, which was consistent with the expected SPV behavior of n-type semiconductors. In addition, a large negative out-of-phase $(Y)$ surface photovoltage signal indicated the slow electron transport towards the FTO back contact of the $\mathrm{TiO}_{2}$ nanorod array (Fig. 3.20(a)). Hence, the $\mathrm{TiO}_{2}$ nanorod array exhibited the smallest overall SPV amplitude among all three samples (Fig. 3.20(d)). For the $\mathrm{TiO}_{2} @ \mathrm{MOF}$ nanorod array (Fig. 3.20(b)), a significant enhancement in the positive in-phase $(X)$ SPV signal was observed along with a decrease in the negative out-of-phase $(Y)$ SPV signal. As a result, the overall SPV amplitude of the $\mathrm{TiO}_{2} @ \mathrm{MOF}$ sample was greater than that of $\mathrm{TiO}_{2}$ alone (Fig. 3.20(d)). This indicated that the MOF coating promoted the charge separation and the charge extraction out of the photoanode. Introduction of cobalt into the MOF ( $\mathrm{TiO}_{2} @ \mathrm{Co}-\mathrm{MOF}$ ) further increased the overall SPV amplitude (Fig. 3.20(d)). 
In addition, the $\mathrm{TiO}_{2} @ \mathrm{MOF}$ and $\mathrm{TiO}_{2} @ \mathrm{Co}-\mathrm{MOF}$ samples showed an evident SPV signal in the visible-light range $(\lambda \geq 440 \mathrm{~nm})$. These surface charges must come from the MOF layer because only the MOF layer can absorb light in this spectral range, which was concluded from the comparison of Fig. 3.20(d) with Fig. 3.17(a). Keeping in mind that the IPCE value in the same spectral range $(\lambda \geq 440 \mathrm{~nm}$ ) was very small (Figs. 3.17(b) and 3.17(c)), it can be concluded that photogenerated charge carriers accounted for a very small portion in the overall photocurrent in the PEC because the MOF layer was too thin ( $8 \mathrm{~nm}$ thick). In short, herein MOF did not act as a photosensitizer. Instead, it just assisted the extraction of the photogenerated carriers out of $\mathrm{TiO}_{2}$ and promoted the injection of these carriers into the electrolyte. The internal electric field at the $\mathrm{p}-\mathrm{n}$ junction reduced the charge recombination rate, as confirmed by the extended $\tau_{\text {rec }}$ (Fig. 3.12(c)) and promoted the charge mobility of the photogenerated charge carriers in the photoanode as shown by the SPV spectra. Coating $\mathrm{TiO}_{2}$ with MOF reduced the trapping of photogenerated holes at the photoanode/electrolyte interface and favored the charge injection into the electrolyte. Incorporation of $\mathrm{Co}$ (III) into MOF further improved the charge mobility of the photogenerated charge carriers in the photoanode and enhanced the charge injection into the electrolyte.
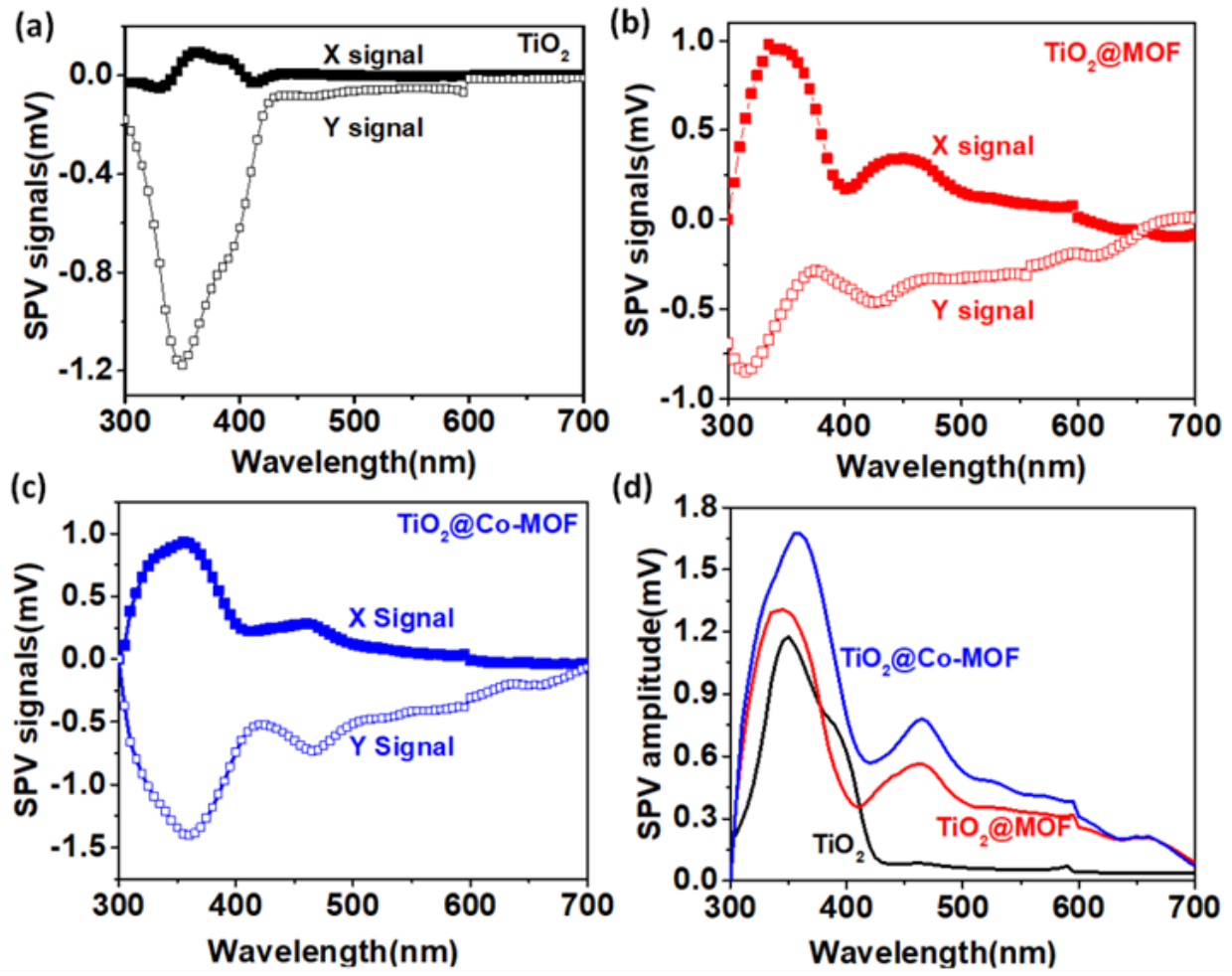

Figure 3.20: Surface photovoltage (SPV) measurement. (a) in-phase (X) and out-of-phase (Y) components of the SPV signal for $\mathrm{TiO}_{2}$ nanorod array on FTO glass; (b) in-phase (X) and out-ofphase (Y) components of the SPV signal for $\mathrm{TiO}_{2} @ \mathrm{MOF}$ nanorod array; (c) in-phase (X) and outof-phase (Y) components of the SPV signal for $\mathrm{TiO}_{2} @$ Co-MOF nanorod array; (d) SPV amplitude comparison of $\mathrm{TiO}_{2}, \mathrm{TiO}_{2} @ \mathrm{MOF}$, and $\mathrm{TiO}_{2} @ \mathrm{Co}-\mathrm{MOF}$ nanorod arrays.

Finally, the stability of the photoanodes during photoelectrochemical operation was tested. The $\mathrm{TiO}_{2}, \mathrm{TiO}_{2} @ \mathrm{MOF}$ and $\mathrm{TiO}_{2} @ \mathrm{Co}-\mathrm{MOF}$ samples were operated continuously at $1.23 \mathrm{~V}$ (vs. RHE) for $3 \mathrm{~h}$ under full spectrum illumination (Fig. 3.21). The test results showed that the 
$\mathrm{TiO}_{2} @ \mathrm{MOF}$ arrays exhibited excellent stability during operation for photoelectrochemical watersplitting. It was observed that bubbles were generated and attached on the surface of $\mathrm{TiO}_{2} @ \mathrm{Co}-$ MOF nanorod photoanode, which caused the noises to the $J-t$ curve. However, a smooth curved was obtained under stirring. SEM images were taken from the $\mathrm{TiO}_{2} @ \mathrm{MOF}$ and $\mathrm{TiO}_{2} @ \mathrm{Co}-\mathrm{MOF}$ samples after the stability tests (Fig. 3.22). The MOF thin films were still covered on the surface of $\mathrm{TiO}_{2}$, which indicated that the MOF layer was stable under irradiation and bias. Given all experiments were performed in neutral electrolyte solutions, this porphyrin-based MOF may be useful for passivation of semiconductor photoanode materials with poor stability in neutral electrolytes, such as $\mathrm{BiVO}_{4}$ and $\mathrm{CuWO}_{4}{ }^{[45,46]}$.

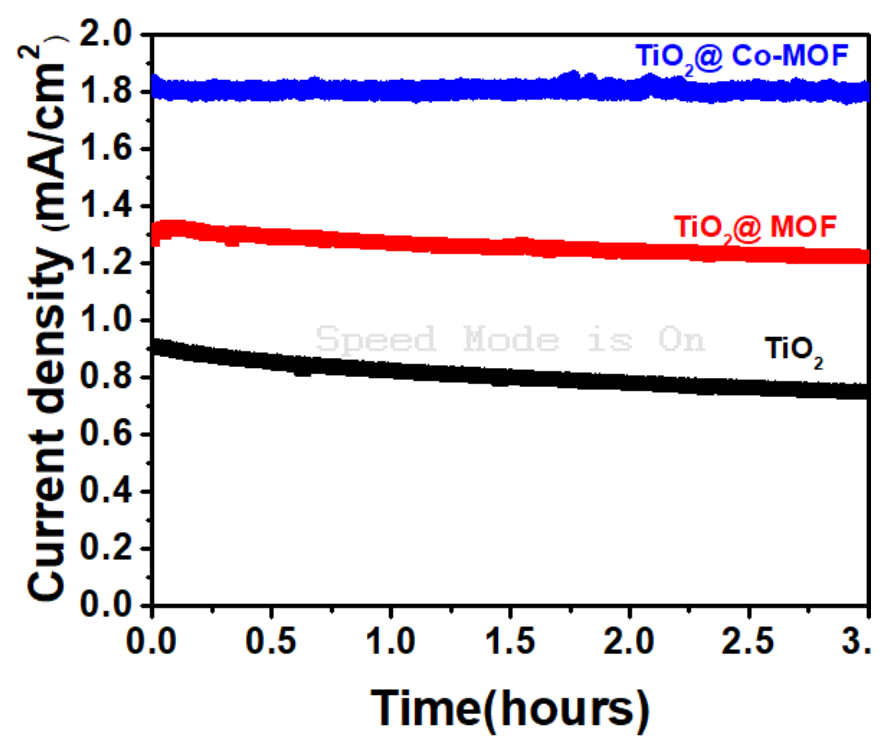

Figure 3.21: Stability tests for different nanorod array photoanodes. (a) $\mathrm{TiO}_{2}$, (b) $\mathrm{TiO}_{2} @ \mathrm{MOF}$, (c) $\mathrm{TiO}_{2} @ \mathrm{Co}-\mathrm{MOF}$.
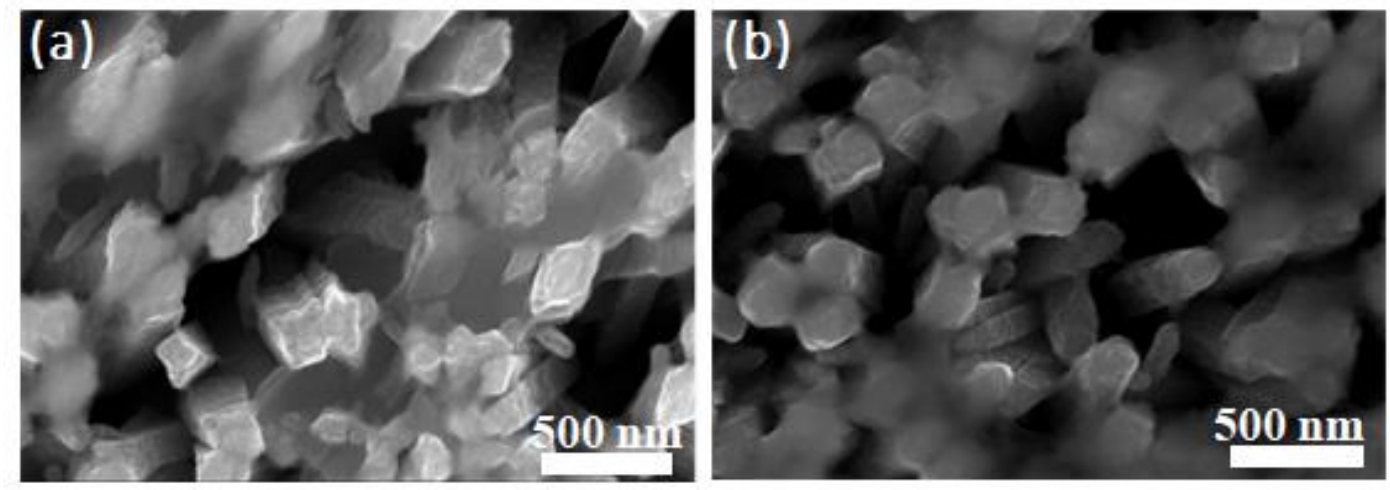

Figure 3.22: $\mathrm{SEM}$ images of (a) $\mathrm{TiO}_{2} @ \mathrm{MOF}$, and (b) $\mathrm{TiO}_{2} @ \mathrm{Co}-\mathrm{MOF}$ samples after stability test. 


\subsection{Conclusions}

In summary, a p-n heterojunction photoanode was formed by coating a conformal thin $\mathrm{p}$ type porphyrin-based MOF or Co-MOF onto the n-type $\mathrm{TiO}_{2}$ nanorod array. The MOF coating and subsequent introduction of $\mathrm{Co}^{3+}$ into the MOF resulted in a 2.7 times enhancement in photocurrent density at $1.23 \mathrm{~V}$ (vs. RHE) with respect to the bare $\mathrm{TiO}_{2}$ nanorod array. The photocurrent enhancement for the $\mathrm{TiO}_{2} @ \mathrm{MOF}$ and $\mathrm{TiO}_{2} @ \mathrm{Co}-\mathrm{MOF}$ has been attributed to multiple factors. Charge separation and hole extraction from $\mathrm{TiO}_{2}$ into the MOF layer was improved due to the built-in electric field at the $\mathrm{p}-\mathrm{n}$ junction between $\mathrm{TiO}_{2}$ and $\mathrm{MOF}$. Also, the MOF coating improved remarkably the charge injection into the electrolyte. Incorporation of $\mathrm{Co}$ (III) into MOF further improved the charge mobility of the photogenerated charge carriers in the photoanode, enhanced the charge injection into the electrolyte. This chapter has demonstrated MOFs have a great promise in solar water-splitting.

\subsection{Methods}

\subsubsection{Materials}

All chemicals were purchased from without further purification. FTO glass and dimethyl sulfoxide (DMSO, 99.5+\%) were purchased from Sigma-Millipore. Titanium n-butoxide (99+\%), hydrochloric acid (36\% w/w), ammonium hydroxide ( $28 \% \mathrm{w} / \mathrm{v} \mathrm{NH}$ ), zirconium(IV) chloride (ZrCl4, 99.5+\%), cobalt(II) chloride hexahydrate $\left(\mathrm{CoCl}_{2} \cdot 6 \mathrm{H}_{2} \mathrm{O}\right.$, ACS, 98\%-102\%), diethylformamide (DEF, anhydrous, 99.8\%), and TEOA (98+\%) were purchased from Alfa-Aesar. Anhydrous sodium sulfate was purchased from Amresco. Tetra-meso(4-carboxyphenyl) porphyrine (TCPP) was purchased from Frontier Scientific.

\subsubsection{Growth of $\mathrm{TiO}_{2}$ nanorod array on FTO}

Oriented rutile $\mathrm{TiO}_{2}$ nanowire arrays were grown on FTO substrate through a hydrothermal method $^{[28]}$. Typically, $1.5 \mathrm{~mL}$ of titanium n-butoxide was dissolved with $60 \mathrm{~mL}$ of $6 \mathrm{M}$ hydrochloric acid. After stirring for $30 \mathrm{~min}$, the solution was transferred into a $125 \mathrm{~mL}$ of Teflonlined stainless-steel autoclave (Parr Instruments 4748), four pieces of pre-cleaned FTO glass substrates $(2.0 \mathrm{~cm} \times 1.25 \mathrm{~cm} \times 2 \mathrm{~mm})$ were placed into the autoclave. The hydrothermal reaction was conducted in an electronic oven at $180{ }^{\circ} \mathrm{C}$ for $2.5 \mathrm{~h}$. After the reaction, the $\mathrm{TiO}_{2}$ covered FTO glass was rinsed with deionized water and ethanol, dried with an air flow, and sintered in a furnace at $450{ }^{\circ} \mathrm{C}$ for $1 \mathrm{~h}$. After sintering, the $\mathrm{TiO}_{2}$ nanorod arrays were further immersed in a "Base Piranha" $\left(\mathrm{H}_{2} \mathrm{O}_{2}: \mathrm{NH}_{3} \cdot \mathrm{H}_{2} \mathrm{O}: \mathrm{H}_{2} \mathrm{O}\right.$ (v:v:v = 1:1:5) solution to improve the their hydrophilicity for subsequent MOF coating. 


\subsubsection{Fabrication of p-n $\mathrm{TiO}_{2} @$ MOF nanorod arrays}

Thin films of porphyrin-based $\mathrm{PCN}-225 \mathrm{MOF}$ on $\mathrm{TiO}_{2}$ nanorod arrays were fabricated using a LbL approach. The LbL approach provides a direct route to MOFs containing metal-free porphyrins. In a typical procedure, the $\mathrm{TiO}_{2}$ nanorod arrays were alternately immersed in a 0.5 $\mathrm{mM}$ TCPP in ethanol solution followed by a $2 \mathrm{mM} \mathrm{ZrCl}_{4}$ in ethanol solution at $40{ }^{\circ} \mathrm{C}$ for $10 \mathrm{~min}$ intervals. The immersion process was subsequently repeated for 5 cycles to form a MOF layer of a given thickness denoted as $\mathrm{TiO}_{2} @ \mathrm{MOF} 5 \mathrm{Cycles}$ for the rest of this manuscript. $\mathrm{TiO}_{2} @ \mathrm{MOF}$ samples were subsequently heated at $150{ }^{\circ} \mathrm{C}$ under an $\mathrm{N}_{2}$ gas environment to promote better contact between the $\mathrm{TiO}_{2}$ nanorods and PCN-225 MOF coating ${ }^{[26,27,30]}$.

\subsection{4 $\mathrm{Co}^{3+}$ functionalization of p-n TiO @MOF nanorod array on FTO}

$\mathrm{Co}^{3+}$ ions were incorporated into the center of TCPP molecule in the PCN-225 MOF structure to form a Co-TCPP complex, synthesized according to the previous literature ${ }^{[47]}$. Briefly, $\mathrm{TiO}_{2} @ \mathrm{MOF}$ nanorod arrays on FTO glass were placed into a $0.24 \mathrm{mM} \mathrm{CoCl}{ }_{2} \cdot 6 \mathrm{H}_{2} \mathrm{O}$ in DMSO solution, followed by refluxing $138{ }^{\circ} \mathrm{C}$ for $12 \mathrm{~h}$. The resulting Co-functionalized $\mathrm{TiO}_{2} @ \mathrm{MOF}$ samples were referred to as $\mathrm{TiO}_{2} @ \mathrm{Co}-\mathrm{MOF}$. After the solution was cooled to room temperature, the $\mathrm{TiO}_{2} @ \mathrm{Co}-\mathrm{MOF}$ on FTO glass was removed, washed with ethanol, and dried by an air flow.

\subsubsection{Synthesis of porphyrin MOF and Co-MOF powder}

$\mathrm{ZrCl}_{4}(70 \mathrm{mg})$, TCPP (50 mg) and benzoic acid (2,700 mg) were added into $8 \mathrm{~mL}$ of DEF, ultrasonically dissolved. The mixture was heated in $120{ }^{\circ} \mathrm{C}$ oven for $48 \mathrm{~h}$. After the reaction, red needle shaped crystals were harvested by filtration $(35 \mathrm{mg}, 47 \% \text { yield })^{[30]}$.

\subsubsection{Characterization}

Field emission scanning electron microscopy (FESEM) images were taken with a JEOL JSM-7600 with an accelerating voltage of $15 \mathrm{kV}$. TEM images were taken with a JEOL JEM 2100F. The UV-Vis absorption spectra were measured by the diffuse-reflection mode with Shimadzu UV-2550 spectrometer equipped with an integrating sphere (UV 2401/2, Shimadzu). XPS measurements were performed with Physical Electronics PHI 5000 Versa Probe to analyze the chemical state and atomic concentrations of elements. Binding energies (BE) were calibrated at adventitious carbon $\mathrm{BE}$ of $284.8 \mathrm{eV}$.

\subsubsection{Photoelectrochemical measurements}

The performance of the PEC was measured with a three-electrode configuration using a Gamry Reference 3000 Potentiostat/Galvanostat/ZRA Instrument. An aqueous electrolyte 
containing $0.2 \mathrm{M} \mathrm{Na}_{2} \mathrm{SO}_{4}(\mathrm{pH}=7)$ was purged with $\mathrm{N}_{2}$ for 30 min prior to measurement. The $\mathrm{TiO}_{2}, \mathrm{TiO}_{2} @ \mathrm{MOF}$ and $\mathrm{TiO}_{2} @ \mathrm{Co}-\mathrm{MOF}$ nanorod arrays were used as the working electrode with a Pt wire as the counter electrode and the $\mathrm{Ag} / \mathrm{AgCl}$ (Sat. $\mathrm{KCl}, E^{\circ}=+0.1976 \mathrm{~V}$ vs. NHE) as the reference electrode. An unfiltered $300 \mathrm{~W}$ Xe lamp was used as the light source. The choice to use an unfiltered Xe lamp was made to preserve UV light at wavelengths below $370 \mathrm{~nm}$ that are cutoff by a traditional AM1.5 filter in PEC experiments ${ }^{[48]}$. As the AM1.5G spectrum does contain non-negligible amounts of light at wavelengths below $370 \mathrm{~nm}$ and the $\mathrm{TiO}_{2}$ nanorod arrays have a significant part of their total light absorption confined to the UV part of the spectrum below 370 $\mathrm{nm}$. The potential vs. RHE was calculated with a reference to $\mathrm{Ag} / \mathrm{AgCl}$ according to the Nernst equation: ${ }^{[43]}$

$$
E_{\mathrm{RHE}}=E_{\mathrm{Ag} / \mathrm{AgCl}}+0.05916 \mathrm{pH}+E_{0},
$$

where $E_{\mathrm{RHE}}$ is the potential vs. RHE, $E_{\mathrm{Ag} / \mathrm{AgCl}}$ is the measured potential vs. $\mathrm{Ag} / \mathrm{AgCl}$, and $E_{0}=$ $0.1976 \mathrm{~V}$ at $25^{\circ} \mathrm{C}$.

\subsubsection{Transient photocurrent density measurements}

Transient photocurrent density measurements were performed at $0 \mathrm{~V}$ vs. $\mathrm{Ag} / \mathrm{AgCl}$ for all samples. The obtained transient photocurrents were then processed to make plots of the natural $\operatorname{logarithm}$ of $D$, a dimensionless parameter calculated by the following equation: ${ }^{[49]}$

$$
\ln (D)=\frac{J(t)-J_{s s}}{J_{i n}-J_{s s}}
$$

where $J(t)$ is the photocurrent density at a given time $(t), J_{\mathrm{ss}}$ is the steady-state photocurrent density at a given time, and $J_{\text {in }}$ is the initial photocurrent density once light illumination begins. The nominal charge recombination lifetime for each sample $\left(\tau_{\text {rec }}\right)$ was taken as the time at which $\ln (D)$ $=-1$.

\subsubsection{Wavelength-dependent IPCE}

IPCE was measured with a 300W Xe lamp with an aligned monochromator (Oriel Cornerstone 130 1/8m). The IPCE was calculated according to Equation (3.4): ${ }^{[50,51]}$

$$
I P C E=\frac{1240 \mathrm{~J}}{\lambda \cdot I_{\text {light }}},
$$

where $J$ is the measured photocurrent density at $+0.6 \mathrm{~V}$ vs. $\mathrm{Ag} / \mathrm{AgCl}$ at a certain wavelength $(\lambda)$, and $I_{\text {light }}$ is the irradiance intensity at the specific wavelength $(\lambda)$. 


\subsubsection{M-S plots}

M-S plots were obtained with a three-electrode cell at an alternating current (AC) frequency of $5 \mathrm{kHz}$ using an AC amplitude of $20 \mathrm{mV}$. The capacitance $(C)$ was calculated from the electrochemical impedance spectroscopy (EIS) spectra at a potential according to Equation $(3.5):^{[50,51]}$

$$
Z_{\text {img }}=\frac{1}{2 \pi f C}
$$

where $Z_{i m g}$ is the imaginary part of the impedance and, $f$ is the frequency, and $C$ is the capacitance. The M-S plots were then generated with the capacitance value normalized with the exposed planar surface area of the electrode. Flat-band potentials were obtained from the M-S plots $\left(1 / C^{2}\right.$ vs. potential) by linearly interpolating the linear region of the M-S plots to its intercept with the potential axis.

\subsubsection{Surface photovoltage spectroscopy}

Surface photovoltage spectroscopy were performed on samples by placing a transparent FTO electrode on the $\mathrm{TiO}_{2}$ and $\mathrm{TiO}_{2} @$ MOF nanorod array on FTO glass samples with air/insulator as a dielectric medium in between sample and FTO electrode to form a parallel plate capacitor. In the case of PCN-225 MOF powder, a small amount of PCN-225 powder was sandwiched between two FTO electrodes. The samples were illuminated with chopped monochromatic light $\left(f_{\text {chop }}=35\right.$ $\mathrm{Hz}$ ) at varying wavelengths. The surface charge is collected through lock-in amplifier (Stanford Research SR830) as in phase $(X)$ and out of phase $(Y)$ signals. The in phase ( $X$-signal) and out of phase ( $Y$-signal, 90 shift) give information about the instantaneous charge collected on the sample surface/quartz + FTO electrode interface and the time response of the charge separation respectively ${ }^{[42]}$. The SPV amplitude is the square root of the sum of the squares of the $X$ and $Y$ signals ${ }^{[52,53]}$.

$$
\text { SPV amplitude }=\left(X^{2}+Y^{2}\right)^{1 / 2},
$$

where $X$ is in-phase signal with respect to modulated input light illumination and $Y$ is out-of-phase signal shifted by $90^{\circ}$ with respect to modulated input light illumination.

\subsubsection{Calculation of charge injection efficiency and charge separation efficiency}

The charge injection efficiency and charge separation efficiency can be evaluated as follows: The total steady-state water oxidation photocurrent density $\left(J_{\text {photocurrent/H2O }}\right)$ generated in the aqueous electrolyte is expressed as:

$$
J_{\text {photocurrent } / H 2 O}=J_{\text {absorb }} \times P_{\text {sep }} \times P_{\text {inject }},
$$

where $J_{\text {absorb }}$ is the photocurrent density that result from an absorbed photon conversion efficiency 
(APCE) of $100 \% . P_{s e p}$ and $P_{\text {inject }}$ are the charge separation efficiency and the charge injection efficiency, respectively.

For oxidation of TEOA, $P_{\text {inject }}$ is considered to be approximately $100 \%$ at all potentials, then:

$$
J_{\text {photocurrent } / T E O A}=J_{\text {absorb }} \times P_{\text {sep }} \text {. }
$$

The charge injection efficiency $P_{\text {inject }}$ for $\mathrm{TiO}_{2}, \mathrm{TiO}_{2} @ \mathrm{MOF}, \mathrm{TiO}_{2} @ \mathrm{Co}-\mathrm{MOF}$ photoanodes can be expressed as:

$$
P_{\text {inject }}=J_{\text {photocurrent/H2O/ }} J_{\text {photocurrent } / T E O A} .
$$

The charge separation efficiency enhancement factor in the presence of MOF coating and Co-MOF coating can be expressed as:

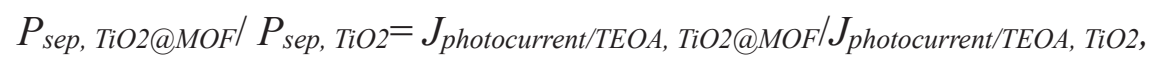

and

$$
P_{\text {sep,TiO2@Co-MOF }} / P_{\text {sep,TiO2 }}=J_{\text {photocurrent/TEOA,Co-TiO2@MOF/ }} J_{\text {photocurrent/TEOA, TiO2. }}
$$

Applied bias photoconversion efficieny (ABPE) represents the photoelectrode performance as a function of the applied potential. The ABPE efficiency is calculated by:

$$
\operatorname{ABPE}(\%)=J_{p h} \frac{\left(1.23 \mathrm{~V}-\mathrm{V}_{\mathrm{app}}\right)}{\rho} \times 100,
$$

where $J_{p h}$ is the measured photocurrent density under AM1.5G illumination, $1.23 \mathrm{~V}$ is the standard state reversible potential of $\mathrm{H}_{2} \mathrm{O}$ oxidation, $V_{\text {app }}$ is the applied potential during the measurement of the photocurrent density versus the reversible hydrogen electrode (RHE) potential, $\rho$ is the total integrated optical power input density $\left(100 \mathrm{~mW} / \mathrm{cm}^{2}\right)$ using AM1.5G illumination (M. Shaban, M. Rabia, A.M.A. El-Sayed, A. Ahmed, S. Sayed, Sci. Rep., 2017, 7, 14100.). J-V curve data under AM1.5G illumination (Figure S13) was used for calculating the APBE.

\subsection{References}

[1] Fujishima, A.; Honda, K. Electrochemical photolysis of water at a semiconductor electrode. Nature 1972, 238, 37-38.

[2] Kang, D.; Kim, T. W.; Kubota, S. R.; Cardiel, A. C.; Cha, H. G.; Choi, K.S. Electrochemical synthesis of photoelectrodes and catalysts for use in solar water splitting. Chem. Rev. 2015, 115, 12839-12887.

[3] Dresselhaus, M. S.; Thomas, I. L. Alternative energy technologies. Nature 2001, 414, 332337. 
[4] Tachibana, Y.; Vayssieres, L.; Durrant, J. R. Artificial photosynthesis for solar watersplitting. Nat. Photonics 2012, 6, 511-518.

[5] Walter, M. G.; Warren, E. L.; McKone, J. R.; Boettcher, S. W.; Mi, Q. X.; Santori, E. A.; Lewis, N. S. Solar water splitting cells. Chem. Rev. 2010, 110, 6446-6473.

[6] Katz, M. J.; Riha, S. C.; Jeong, N. C.; Martinson, A. B. F.; Farha, O. K.; Hupp, J. T. Toward solar fuels: Water splitting with sunlight and "rust"? Coord. Chem. Rev. 2012, 256, 2521-2529.

[7] Mayer, M. T.; Du, C.; Wang, D. W. Hematite/si nanowire dual-absorber system for photoelectrochemical water splitting at low applied potentials. J. Am. Chem. Soc. 2012, $134,12406-12409$.

[8] Chernomordik, B. D.; Russell, H. B.; Cvelbar, U.; Jasinski, J. B.; Kumar, V.; Deutsch, T.; Sunkara, M. K. Photoelectrochemical activity of as-grown, $\alpha-\mathrm{Fe} 2 \mathrm{O} 3$ nanowire array electrodes for water splitting. Nanotechnology 2012, 23, 194009.

[9] Osterloh, F. E. Inorganic nanostructures for photoelectrochemical and photocatalytic water splitting. Chem. Soc. Rev. 2013, 42, 2294-2320.

[10] Zhang, Y. H.; Tang, Z. R.; Fu, X. Z.; Xu, Y. J. TiO2-graphene nanocomposites for gasphase photocatalytic degradation of volatile aromatic pollutant: Is TiO2-graphene truly different from other TiO2-carbon composite materials? ACS Nano 2010, 4, 7303-7314.

[11] Zhou, H. C.; Long, J. R.; Yaghi, O. M. Introduction to metal-organic frameworks. Chem. Rev. 2012, 112, 673-674.

[12] Xia, W.; Mahmood, A.; Zou, R. Q.; Xu, Q. Metal-organic frameworks and their derived nanostructures for electrochemical energy storage and conversion. Energy Environ. Sci. 2015, 8, 1837-1866.

[13] Wang, W.; Xu, X. M.; Zhou, W.; Shao, Z. P. Recent progress in metal-organic frameworks for applications in electrocatalytic and photocatalytic water splitting. Adv. Sci. 2017, 4, 1600371.

[14] Kuc, A.; Enyashin, A.; Seifert, G. Metal-organic frameworks: Structural, energetic, electronic, and mechanical properties. J. Phys. Chem. B 2007, 111, 8179-8186.

[15] Zhang, P.; Guan, B. Y.; Yu, L.; Lou, X. W. Facile synthesis of multi-shelled ZnS-CdS cages with enhanced photoelectrochemical performance for solar energy conversion. Chem 2018, 4, 162-173.

[16] Zhang, H. B.; Nai, J. W.; Yu, L.; Lou, X. W. Metal-organic-framework-based materials as platforms for renewable energy and environmental applications. Joule 2017, 1, 77-107.

[17] Wu, H. B.; Lou, X. W. Metal-organic frameworks and their derived materials for electrochemical energy storage and conversion: Promises and challenges. Sci. Adv. 2017, 3, eaap9252. 
[18] Wang, W.; Xu, X. M.; Zhou, W.; Shao, Z. P. Recent progress in metal-organic frameworks for applications in electrocatalytic and photocatalytic water splitting. Adv. Sci. 2017, 4, 1600371.

[19] Zhang, L. P.; Cui, P.; Yang, H. B.; Chen, J. Z.; Xiao, F. X.; Guo, Y. Y.; Liu, Y.; Zhang, W. N.; Huo, F. W.; Liu, B. Metal-organic frameworks as promising photosensitizers for photoelectrochemical water splitting. Adv. Sci. 2016, 3, 1500243.

[20] Dou, Y. B.; Zhou, J.; Zhou, A. W.; Li, J. R.; Nie, Z. R. Visible-light responsive MOF encapsulation of noble-metal-sensitized semiconductors for high-performance photoelectrochemical water splitting. J. Mater. Chem. A 2017, 5, 19491-19498.

[21] Otsuki, J. Supramolecular approach towards light-harvesting materials based on porphyrins and chlorophylls. J. Mater. Chem. A 2018, 6, 6710-6753.

[22] Gao, W. Y.; Chrzanowski, M.; Ma, S. Q. Metal-metalloporphyrin frameworks: A resurging class of functional materials. Chem. Soc. Rev. 2014, 43, 5841-5866.

[23] Zhao, M.; Ou, S.; Wu, C. D. Porous metal-organic frameworks for heterogeneous biomimetic catalysis. Acc. Chem. Res. 2014, 47, 1199-1207.

[24] Huh, S.; Kim, S. J.; Kim, Y. Porphyrinic metal-organic frameworks from custom-designed porphyrins. CrystEngComm 2016, 18, 345-368.

[25] Farha, O. K.; Shultz, A. M.; Sarjeant, A. A.; Nguyen, S. T.; Hupp, J. T. Active-site accessible, porphyrinic metal-organic framework materials. J. Am. Chem. Soc. 2011, 133, $5652-5655$.

[26] Deria, P.; Bury, W.; Hupp, J. T.; Farha, O. K. Versatile functionalization of the NU-1000 platform by solvent-assisted ligand incorporation. Chem. Commun. 2014, 50, 1965-1968.

[27] So, M. C.; Jin, S. Y.; Son, H. J.; Wiederrecht, G. P.; Farha, O. K.; Hupp, J. T. Layer-bylayer fabrication of oriented porous thin films based on porphyrin-containing metalorganic frameworks. J. Am. Chem. Soc. 2013, 135, 15698-15701.

[28] Liu, B.; Aydil, E. S. Growth of oriented single-crystalline rutile TiO2 nanorods on transparent conducting substrates for dye-sensitized solar cells. J. Am. Chem. Soc. 2009, 131, 3985-3990.

[29] Li, J. T.; Hoffmann, M. W. G.; Shen, H.; Fabrega, C.; Prades, J. D.; Andreu, T.; HernandezRamirez, F.; Mathur, S. Enhanced photoelectrochemical activity of an excitonic staircase in $\mathrm{CdS} @ \mathrm{TiO}_{2}$ and CdS@anatase@ $@$ rutile $\mathrm{TiO}_{2}$ heterostructures. J. Mater.Chem. 2012, 22, 20472-20476.

[30] Jiang, H. L.; Feng, D. W.; Wang, K. C.; Gu, Z. Y.; Wei, Z. W.; Chen, Y. P.; Zhou, H. C. An exceptionally stable, porphyrinic $\mathrm{Zr}$ metal-organic framework exhibiting $\mathrm{pH}$-dependent fluorescence. J. Am. Chem. Soc. 2013, 135, 13934-13938.

[31] Yang, H.; Zhang, S. L.; Han, L. H.; Zhang, Z.; Xue, Z.; Gao, J.; Li, Y. J.; Huang, C. H.; Yi, Y. P.; Liu, H. B. et al. High conductive two-dimensional covalent organic framework for lithium storage with large capacity. ACS Appl. Mater. Interfaces 2016, 8, 5366-5375. 
[32] Sarno, D. M.; Matienzo, L. J.; Jones, W. E. X-ray photoelectron spectroscopy as a probe of intermolecular interactions in porphyrin polymer thin films. Inorg. Chem. 2001, 40, 6308-6315.

[33] Fidalgo-Marijuan, A.; Barandika, G.; Bazán, B.; Urtiaga, M. K.; Arriortua, M. I. Selfassembly of iron TCPP (meso-tetra(4-carboxyphenyl)porphyrin) into a chiral 2D coordination polymer. Polyhedron 2011, 30, 2711-2716.

[34] Sonkar, P. K.; Prakash, K.; Yadav, M.; Ganesan, V.; Sankar, M.; Gupta, R.; Yadav, D. K. $\mathrm{Co}(\mathrm{II})$-porphyrin-decorated carbon nanotubes as catalysts for oxygen reduction reactions: An approach for fuel cell improvement. J. Mater. Chem. A 2017, 5, 6263-6276.

[35] Meng, F. K.; Li, J. T.; Cushing, S. K.; Zhi, M. J.; Wu, N. Q. Solar hydrogen generation by nanoscale $\mathrm{p}-\mathrm{n}$ junction of p-type molybdenum disulfide/ n-type nitrogen-doped reduced graphene oxide. J. Am. Chem. Soc. 2013, 135, 10286-10289.

[36] Kim, T. W.; Choi, K. S. Nanoporous BiVO4 photoanodes with dual-layer oxygen evolution catalysts for solar water splitting. Science 2014, 343, 990-994.

[37] Zhou, M.; Bao, J.; Bi, W. T.; Zeng, Y. Q.; Zhu, R.; Tao, M. S.; Xie, Y. Efficient water splitting via a heteroepitaxial BiVO4 photoelectrode decorated with Co-Pi catalysts. ChemSusChem 2012, 5, 1420-1425.

[38] Shaban, M.; Rabia, M.; El-Sayed, A. M. A.; Ahmed, A.; Sayed, S. Photocatalytic properties of $\mathrm{PbS}$ /graphene oxide/polyaniline electrode for hydrogen generation. Sci. Rep. 2017, 7, 14100.

[39] Li, J. T.; Wu, N. Q. Semiconductor-based photocatalysts and photoelectrochemical cells for solar fuel generation: A review. Catal. Sci. Technol. 2015, 5, 1360-1384.

[40] Wang, W. H.; Dong, J. Y.; Ye, X. Z.; Li, Y.; Ma, Y. R.; Qi, L. M. Heterostructured TiO2 nanorod@nanobowl arrays for efficient photoelectrochemical water splitting. Small 2016, 12, 1469-1478.

[41] Hwang, Y. J.; Hahn, C.; Liu, B.; Yang, P. D. Photoelectrochemical properties of TiO2 nanowire arrays: A study of the dependence on length and atomic layer deposition coating. ACS Nano 2012, 6, 5060-5069.

[42] Kronik, L.; Shapira, Y. Surface photovoltage phenomena: Theory, experiment, and applications. Surf. Sci. Rep. 1999, 37, 1-206.

[43] Goodman, A. M. A method for the measurement of short minority carrier diffusion lengths in semiconductors. J. Appl. Phys. 1961, 32, 2550-2552.

[44] Lagowski, J. Semiconductor surface spectroscopies: The early years. Surf. Sci. 1994, 299_ 300, 92-101.

[45] Zhong, D. K.; Choi, S.; Gamelin, D. R. Near-complete suppression of surface recombination in solar photoelectrolysis by "Co-Pi" catalyst-modified W:BiVO4. J. Am. Chem. Soc. 2011, 133, 18370-18377. 
[46] Yourey, J. E.; Pyper, K. J.; Kurtz, J. B.; Bartlett, B. M. Chemical stability of CuWO4 for photoelectrochemical water oxidation. J. Phys. Chem. C 2013, 117, 8708-8718.

[47] Nakazono, T.; Parent, A. R.; Sakai, K. Cobalt porphyrins as homogeneous catalysts for water oxidation. Chem. Commun. 2013, 49, 6325-6327.

[48] Abdi, F. F.; van de Krol, R. Nature and light dependence of bulk recombination in Co-Picatalyzed $\mathrm{BiVO}_{4}$ photoanodes. J. Phys. Chem. C 2012, 116, 9398-9404.

[49] Li, J. T.; Cushing, S. K.; Zheng, P.; Senty, T.; Meng, F. K.; Bristow, A. D.; Manivannan, A.; Wu, N. Q. Solar hydrogen generation by a CdS-Au-TiO2 sandwich nanorod array enhanced with Au nanoparticle as electron relay and plasmonic photosensitizer. J. Am. Chem. Soc. 2014, 136, 8438-8449.

[50] Li, J. T.; Cushing, S. K.; Zheng, P.; Meng, F. K.; Chu, D.; Wu, N. Q. Plasmon-induced photonic and energy-transfer enhancement of solar water splitting by a hematite nanorod array. Nat. Commun. 2013, 4, 2651.

[51] Schmuki, P.; Böhni, H.; Bardwell, J. A. In situ characterization of anodic silicon oxide films by Ac impedance measurements. J. Electrochem. Soc. 1995, 142, 1705-1712.

[52] Wafula, H.; Juma, A.; Sakwa, T.; Musembi, R.; Simiyu, J. A surface photovoltage study of surface defects on Co-doped $\mathrm{TiO} 2$ thin films deposited by spray pyrolysis. Coatings 2016, 6,30 .

[53] Ivanov, T.; Donchev, V.; Germanova, K.; Kirilov, K. A vector model for analysing the surface photovoltage amplitude and phase spectra applied to complicated nanostructures. J. Phys. D: Appl. Phys. 2009, 42, 135302. 


\section{Chapter 4: Rational Design for a Photoelectrochemical (PEC) Sensor Utilizing Plasmonic Energy Transfer for $\mathrm{Hg}^{2+}$ Detection}

\subsection{Introduction}

With the population of Earth expected to exceed 10 billion by $2100^{[1]}$, there is an increasing need for food, safe drinking water, and improved health care to meet societal demands. To aid in dealing with these challenges, accurate detection and quantification of environmental pollutants and biological markers for health diagnostics is needed. Sensors are commonly based on fluorescence, surface enhanced Raman scattering (SERS), or photoelectrochemistry (PEC) as actuation mechanisms. PEC-based sensors where the presence of an analyte modulates photocurrent or photovoltage are of interest due to their low instrumentation cost, low background signals, and simplicity in operation versus established methods like gas and liquid chromatographies..$^{[2-5]}$

One area of research that has applications in sensing is plasmonics. In plasmonics, light illumination triggers the coherent oscillation of conduction band electrons (called surface plasmon resonance (SPR) of nanoparticles of certain metals (e.g. $\mathrm{Ag}, \mathrm{Au}$, and $\mathrm{Cu})^{[6-10]}$ and defective semiconductors (e.g. $\mathrm{MoO}_{3-\mathrm{x}}, \mathrm{WO}_{3-\mathrm{x}}, \mathrm{Cu}_{2-\mathrm{x}} \mathrm{S}, \mathrm{Cu}_{2-\mathrm{x}} \mathrm{Se}, \mathrm{Cu}_{2-\mathrm{x}} \mathrm{Te}$ ). ${ }^{[1-13]}$ Focus is paid to localized surface plasmon resonance (LSPR) that occurs when the plasmon is confined to the surface of extremely small nanoparticles. The light energy stored in the LSPR of these materials can be utilized in multiple ways to increase the photocurrent of a nearby semiconductor. Depending on the size and morphology of the plasmonic nanoparticles, the light energy stored in the plasmon can be radiatively scattered to improve effective optical pathlengths ${ }^{[10,14]}$ or concentrated into intense local electromagnetic fields within the semiconductor ${ }^{[7,10,15]}$. While the LSPR of the plasmonic nanoparticle is coherent $(\mathrm{t}<30 \mathrm{fs}$ ), energy from the plasmon can also be transferred by plasmon-induced resonant energy transfer (PIRET) via dipole-dipole interactions to a semiconductor acceptor. ${ }^{[16-19]}$ After the LSPR of a plasmonic nanoparticle loses coherence, a population of energetic "hot" carriers (electrons and holes) remains that can be transferred to another material and/or directly drive chemical reactions before the hot carriers relax to their ground state. ${ }^{[20-24]}$ All of these mechanisms can be utilized to increase the photocurrent of semiconductor based PEC cells for applications including sensing ${ }^{[25-30]}$. Additional mechanisms where energy is transferred from a semiconductor into plasmonic nanoparticles such as Förster resonance energy transfer (FRET) can also be used to decrease the photocurrent of a nearby semiconductor in a manner that can be used for sensing. ${ }^{[31-33]}$

Several PEC-based sensors use plasmonic nanoparticles in their design in order to modulate photocurrent. Specifically focusing on PEC-based sensors that increase in photocurrent 
in the presence of an analyte, insufficient attention is given towards optimizing the energy transfer mechanism between the plasmonic nanoparticle. Most PEC-based sensors that utilize plasmonic nanoparticles to increase photocurrent are based on hot electron injection. ${ }^{[25-]}$ However, the maximum theoretical efficiency for hot electron injection is limited to $\sim 10 \%$ due to energetics and momentum mismatches between the plasmonic hot carriers and the contacting semiconductor. ${ }^{[34]}$ PIRET is rarely used in PEC-based sensors with limited examples ${ }^{[27]}$ available in literature despite potential energy transfer efficiencies theoretically being as high as $30 \% \cdot{ }^{[35]}$ In addition, to the authors' knowledge, no papers directly attempt to compare and contrast PIRET and hot electron transfer as photocurrent enhancement mechanisms in PEC-based sensors using the same semiconductor film.

In this chapter, we compare hot electron transfer and PIRET from plasmonic Au and $\mathrm{Au} @ \mathrm{SiO}_{2}$ core@ shell nanoparticles (NPs) to a thin $\mathrm{Bi}_{3} \mathrm{FeMo}_{2} \mathrm{O}_{12}$ (BFMO) semiconductor film and explore the potential use of BFMO-plasmonic nanoparticle conjugates as PEC-based sensors for mercury (II) $\left(\mathrm{Hg}^{2+}\right)$ ion detection. Plasmonic nanoparticles (NPs) were controllably linked to the BFMO thin film by use of mismatched single-strand DNA (ssDNA) that could be linked in the presence of $\mathrm{Hg}^{2+}$ as an analyte by formation of double-strand DNA (dsDNA). The nature of the energy transfer between the plasmonic nanoparticles and the BFMO film was tuned by the presence of a dielectric silica $\left(\mathrm{SiO}_{2}\right)$ spacer layer.

\subsection{Results}

The main purpose of this chapter is to demonstrate and compare different plasmon-based energy transfer mechanisms and evaluate their potential for use in PEC sensors. Different pairings of plasmonic nanoparticles with $\mathrm{Bi}_{3} \mathrm{FeMo}_{2} \mathrm{O}_{12}$ (BFMO) films are studied. Plasmonic nanoparticles are bound to the BFMO thin films on FTO glass (50-80 nm thick; see Fig. 4.1) By utilizing $\mathrm{Hg}^{2+}$ ions affinity for thymine, $\mathrm{Hg}^{2+}$ can be selectively bound to ssDNA strands on the BFMO thin films and used to bind to the thymine bases on the complementary ssDNA strands on the plasmonic nanoparticles as shown in the schematic in Fig. 4.2. After a washing step to remove nonspecifically bound plasmonic nanoparticles from the BFMO thin film substrate, the remaining bound plasmonic nanoparticles must be linked to the BFMO through the newly conjugated double stranded DNA (dsDNA). This ensures that plasmonic energy transfer that can increase the overall photocurrent of the BFMO based photoelectrode occurs only when $\mathrm{Hg}^{2+}$ is present with the number of plasmonic nanoparticles bound being proportional to the amount of $\mathrm{Hg}^{2+}$ present in the test sample. 


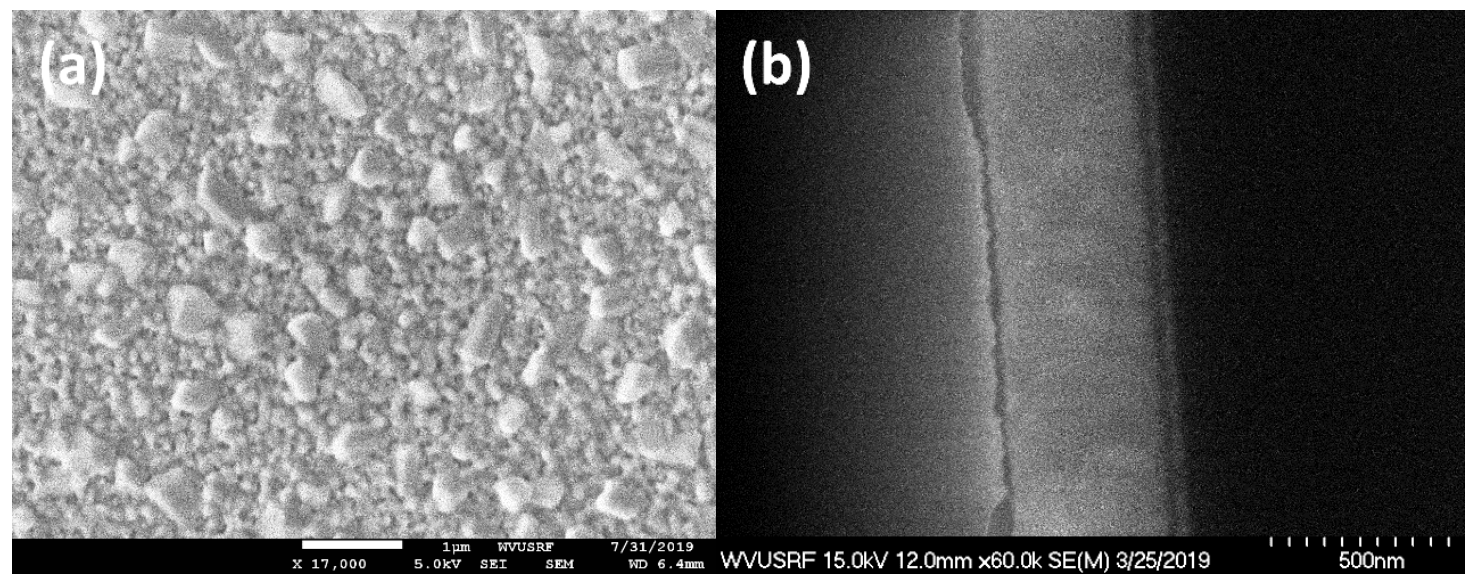

Figure 4.1: SEM Images of $\mathrm{Bi}_{3} \mathrm{FeMo}_{2} \mathrm{O}_{12}(\mathrm{BFMO})$ thin film; (a) Top-down SEM; (b) CrossSection SEM Image of BFMO thin film.

(a) $\mathrm{Hg}^{2+}$ Containing Sample Added to BFMO-DNA Film

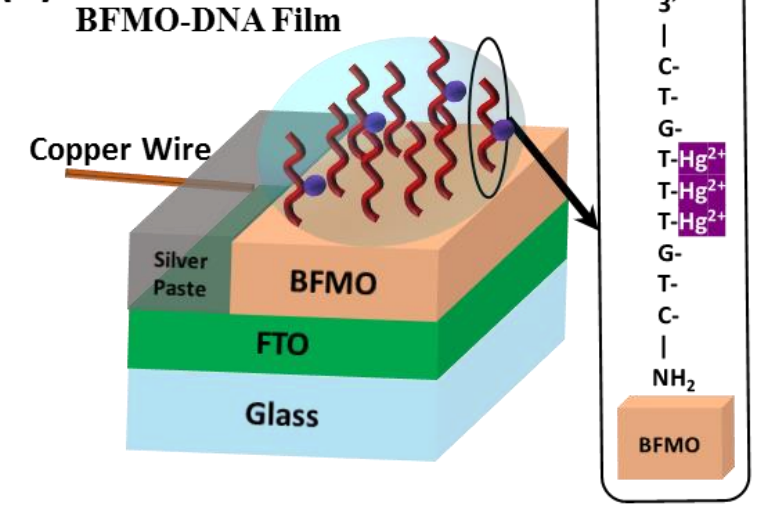

(b) Plasmonic Capture Probes Bind with $\mathrm{Hg}^{2+}$ Functionalized BFMO-DNA Substrate

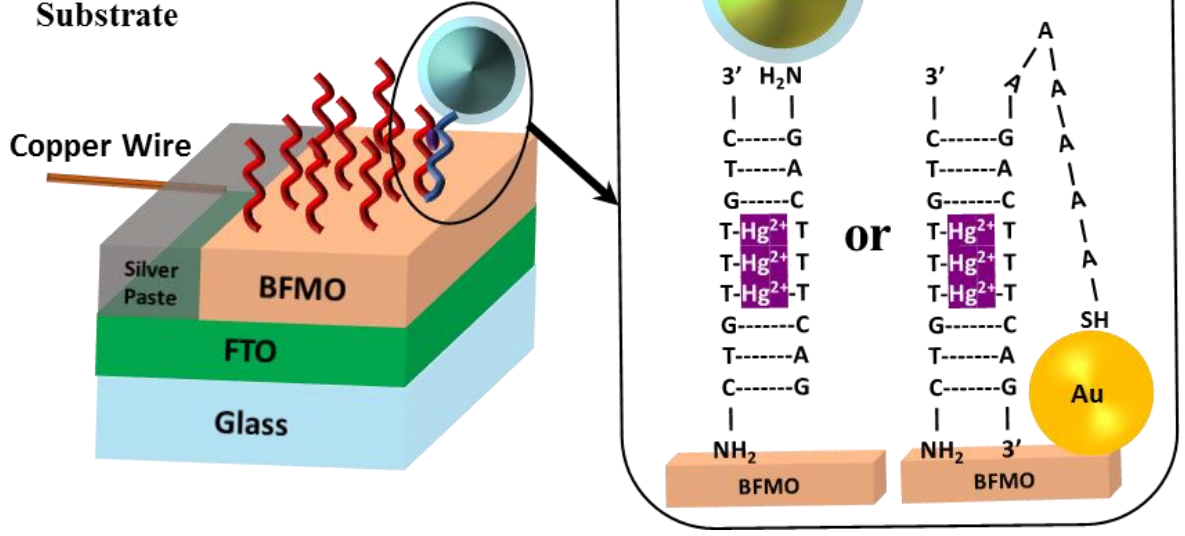

Figure 4.2: Schematic of Plasmonic Nanoparticle Binding to $\mathrm{Bi}_{3} \mathrm{FeMo}_{2} \mathrm{O}_{12}(\mathrm{BFMO})$ Films for Use as a PEC Sensor; (a) $\mathrm{Hg}^{2+}$ conjugation with ssDNA on BFMO film; (b) Conjugation of plasmonic nanoparticles with $\mathrm{Hg}^{2+}$ conjugated BFMO film. 
Figure 4.3 shows the UV-Visible light absorption spectra for the BFMO films and the different types of plasmonic nanoparticles (Au NPs, and $\mathrm{Au} @ \mathrm{SiO}_{2} \mathrm{NPs}$ ) studied in this chapter.

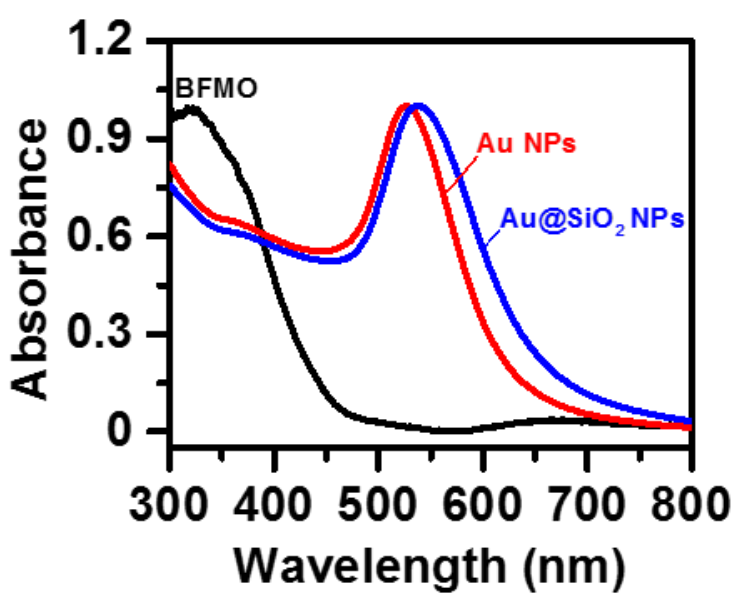

Figure 4.3: Normalized UV-Visible Light Absorption Spectra of $\mathrm{Bi}_{3} \mathrm{FeMo}_{2} \mathrm{O}_{12}$ (BFMO) films, $\mathrm{Au}$, and $\mathrm{Au} @ \mathrm{SiO}_{2} \mathrm{NPs}$.

The main overriding requirement for PIRET from a plasmonic metal to a semiconductor acceptor is a spectral overlap between the LSPR of the plasmonic metal and the absorption spectrum of the semiconductor film with the plasmonic metal's LSPR red-shifted relative to the absorption edge of the semiconductor acceptor ${ }^{[16,17]}$. As seen from Fig. 4.3, the LSPRs of the Au $\mathrm{NPs}$ and $\mathrm{Au} @ \mathrm{SiO}_{2} \mathrm{NPs}$ and BFMO absorption spectrum have a spectral overlap. However, the spectral overlap between the Au NPs and BFMO is limited to only BFMO's absorption tail between 480 - $550 \mathrm{~nm}$ and part of the BFMO absorption edge between $440-480 \mathrm{~nm}$ based on extrapolation of the $\mathrm{Au}$ and $\mathrm{Au} @ \mathrm{SiO}_{2}$ LSPRs. The limited spectral overlap means that the PIRET between the Au NPs and BFMO would be weak due to the weak dipole strength of the BFMO at the absorption tail wavelengths.

(a) BFMO-Au

PIRET, Hot Electrons, \&

Fermi Level Equilibration

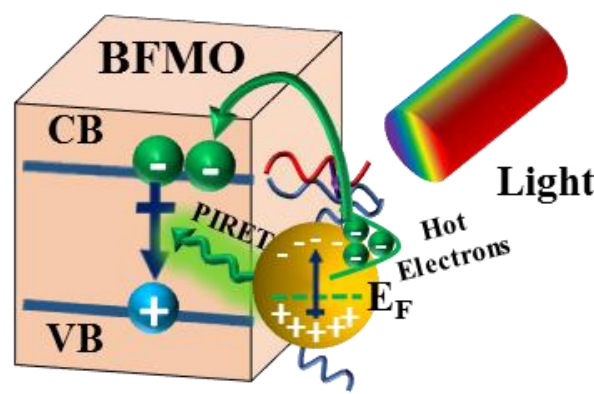

(b) BFMO-Au@ $\mathrm{SiO}_{2}$ PIRET Only

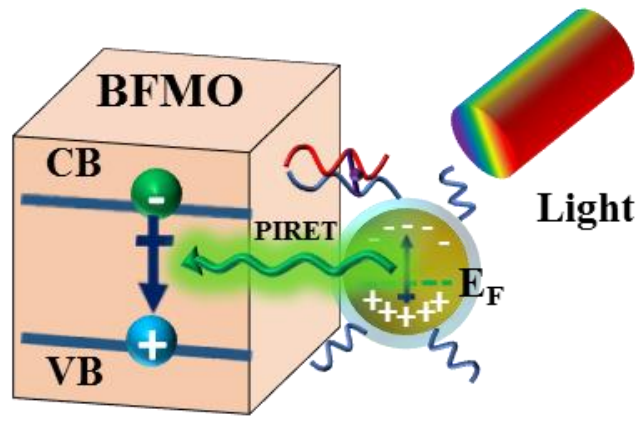

Figure 4.4: Schematic of photocurrent enhancement for the different BFMO - DNA - Plasmonic Nanoparticle combinations studied; (a) BFMO-DNA-Au, (b) BFMO-DNA-Au@ $\mathrm{SiO}_{2}$. 
In the case of $\mathrm{Au}$ NPs without a $\mathrm{SiO}_{2}$ spacer layer (Figure 4.4(a)), hot electron transfer from the $\mathrm{Au}$ NPs to the BFMO is possible in addition to PIRET because of the intimate contact between the plasmonic Au and BFMO and the favorable energetics of the BFMO conduction band and the hot electrons of the Au NPs. ${ }^{[36,37]}$ The presence of a $5 \mathrm{~nm} \mathrm{SiO} 2$ spacer layer on the outside of the $\mathrm{Au} @ \mathrm{SiO}_{2} \mathrm{NPs}$ (Figure 4.5(b)) limits the plasmonic energy transfer to PIRET with the BFMO thin film without any hot electron transfer (Figure 4.4(b)).

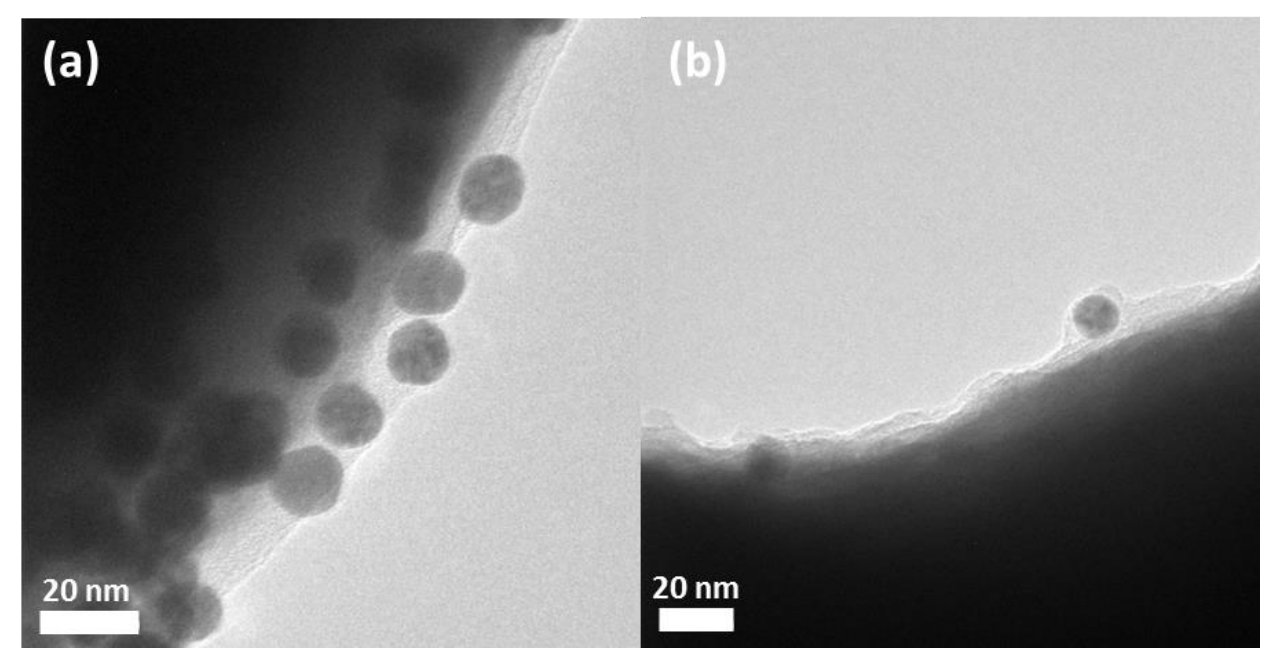

Figure 4.5: HRTEM Images of (a) Au NPs and (b) $\mathrm{Au} @ \mathrm{SiO}_{2} \mathrm{NPs}$

To understand the nature of the energy transfer between the plasmonic nanoparticles and the BFMO films, photoelectrochemical (PEC) testing of the BFMO-DNA films before and after incubation with a solution containing $500 \mathrm{ppb}$ of $\mathrm{Hg}^{2+}$ ions and then plasmonic nanoparticles was performed. Figure 4.6 shows J-V curves and M-S plots when Au NPs and Au@ $\mathrm{SiO}_{2} \mathrm{NPs}$ were the conjugated plasmonic nanoparticles. 

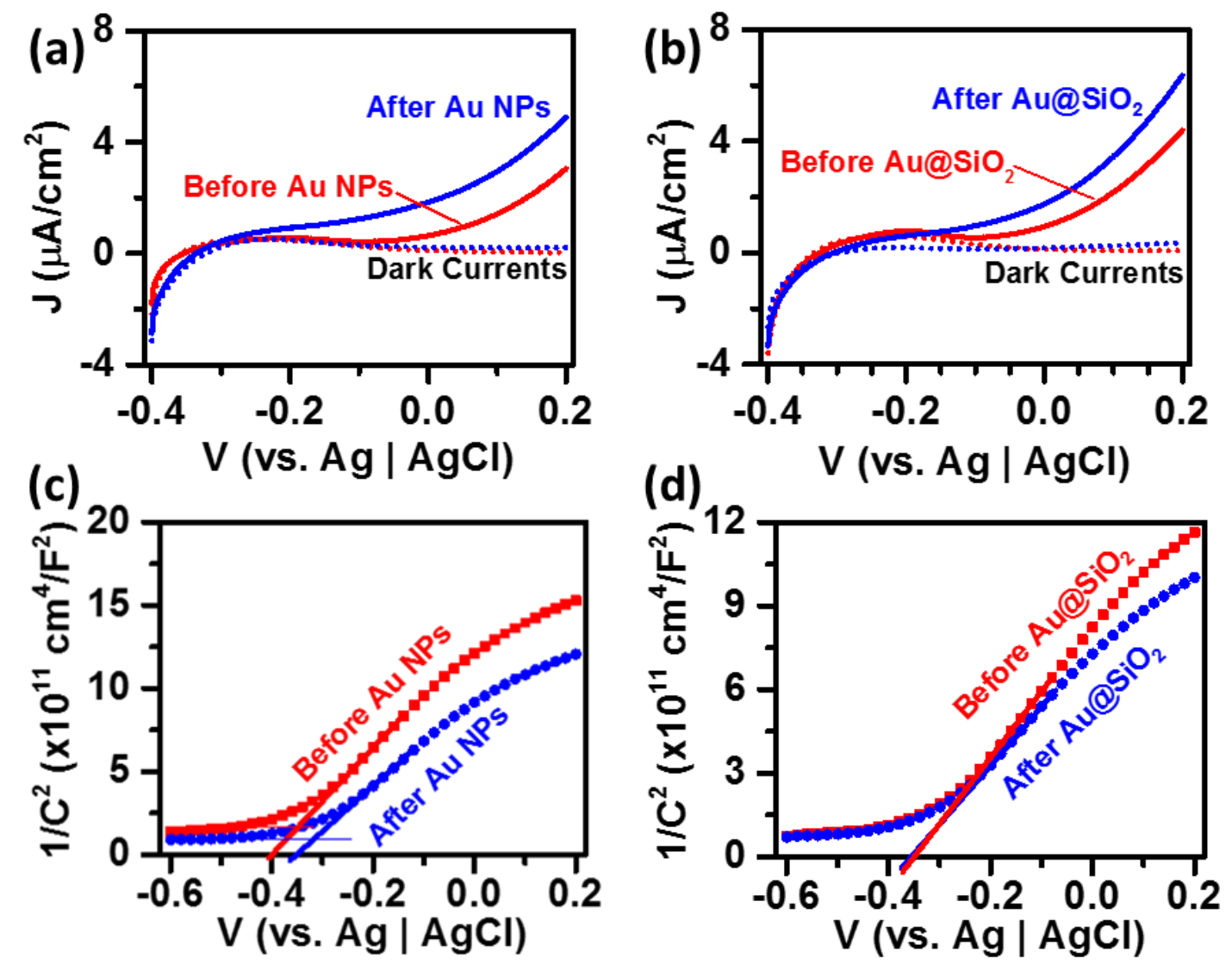

Figure 4.6: (a-b) J-V Curves and (c-d) M-S Plots of BFMO-DNA films before and after incubation with $500 \mathrm{ppb}$ of $\mathrm{Hg}^{2+}$ and plasmonic nanoparticle addition; with $(\mathrm{a}, \mathrm{c}) \mathrm{Au}$ NPs added, and (b, d) $\mathrm{Au} @ \mathrm{SiO}_{2} \mathrm{NPs}$ added.

The addition of $\mathrm{Au}$ NPs (Figure 4.6(a)), and $\mathrm{Au} @ \mathrm{SiO}_{2} \mathrm{NPs}$ (Figure 4.6(b)) result in enhanced photocurrent of the sample. However, there is a negative shift in the photocurrent onset potential +0.05 V shift in flat-band potential on the M-S plot (Figure 4.6(c)) for the BFMO-DNA$\mathrm{Au}$ sample that indicates a change in the Fermi level of the sample after the Au NPs have been added to the sample. The shifts in photocurrent onset and flat-band potentials are consistent with Fermi level equilibration between the BFMO and the contacting Au NPs as a contributing mechanism towards the photocurrent enhancement by the Au NPs ${ }^{[38,39]}$. With Au@SiO $2 \mathrm{NPs}$ added, there is no change in the photocurrent onset potential or the flat-band potential (Figure 4.6(d)). The lack of change in photocurrent onset potential and flat-band potential indicates that the $\mathrm{SiO}_{2}$ shell does not improve the catalytic activity of the BFMO-DNA-Au@ $\mathrm{SiO}_{2} \mathrm{samples}$ within the potential window tested and that charge transfer between the $\mathrm{Au}$ cores has been successfully blocked by the $\mathrm{SiO}_{2}$ shell layers. To separate wavelength-independent enhancement mechanisms like Fermi level equilibration and wavelength-dependent photocurrent enhancement mechanisms, incident photon-to-current efficiency (IPCE) (Figure 4.7) measurements were performed at $+0.15 \mathrm{~V}$ vs. $\mathrm{Ag} \mid \mathrm{AgCl}$ using $500 \mathrm{ppb} \mathrm{Hg}^{2+}$ for plasmonic nanoparticle conjugation. 

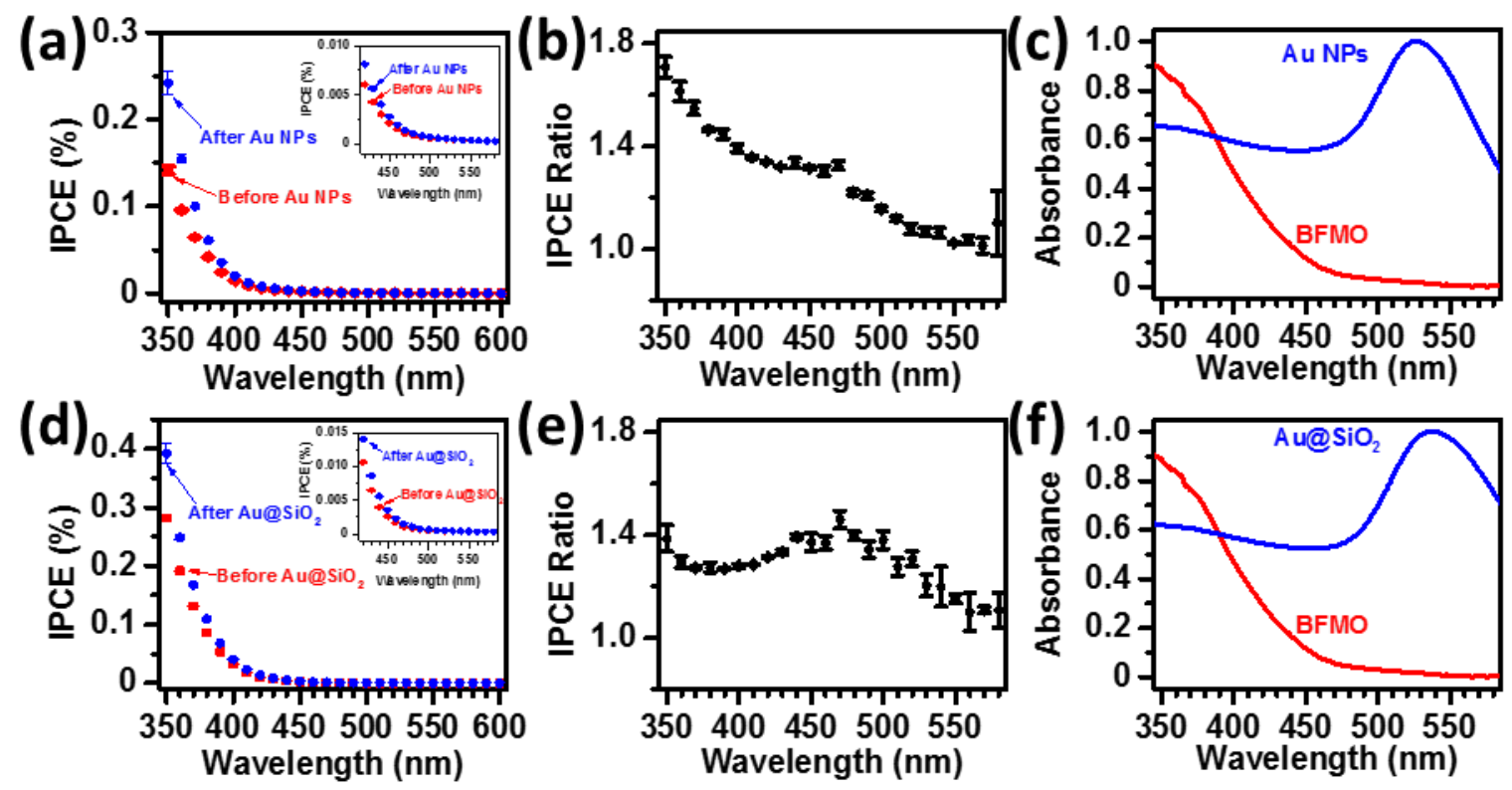

Figure 4.7: (a,d) IPCE Spectra measured at +0.15 V vs. $\mathrm{Ag} \mid \mathrm{AgCl}$ for BFMO-DNA films before and after incubation with $500 \mathrm{ppb}$ of $\mathrm{Hg}^{2+}$ and plasmonic nanoparticle addition, Insets: magnified IPCE spectra between $420 \mathrm{~nm}$ and $580 \mathrm{~nm}$. (b, e) Ratio of IPCE Spectra (After versus Before $\mathrm{Hg}^{2+}$ and plasmonic nanoparticle incubation) and (c,f) normalized UV-Vis absorption spectra for BFMO and corresponding plasmonic nanoparticles: (a-c) with $\mathrm{Au} \mathrm{NPs,} \mathrm{(d-f)} \mathrm{with} \mathrm{Au} @ \mathrm{SiO}_{2} \mathrm{NPs}$.

The overall IPCEs of the BFMO film before incubation with $\mathrm{Hg}^{2+}$ and plasmonic nanoparticles (Figs. 4.7(a) and 4.7(d)) show an onset at $470 \mathrm{~nm}$, consistent with the band edge of the BFMO thin film shown in Figure 1a. Upon incubation with $500 \mathrm{ppb} \mathrm{Hg}^{2+}$ and Au NPs (Figs. 4.7(a) and 4.7(b)) or Au @ $\mathrm{SiO}_{2}$ NPs (Figs. 4.7(d) and 4.7(e)), there is a noticeable increase in the IPCE at wavelengths less than or equal to $550 \mathrm{~nm}$. The $550 \mathrm{~nm}$ onset for IPCE enhancement is consistent with the overlap between weak absorption tail of the BFMO film and the LSPRs of the $\mathrm{Au}$ NPs. The IPCE enhancement can be split into two separate spectral regions. The first region between However, while there is an IPCE at the wavelengths between 480 and $550 \mathrm{~nm}$ for both the BFMO-Au NPs case and BFMO-Au@ $\mathrm{SiO}_{2} \mathrm{NPs}$ case, the overall IPCEs are still under $0.005 \%$. In the case of BFMO-Au films, the enhancement within this spectral region is a combination of hot electron injection, PIRET, and Fermi level equilibration from the Au NPs to the BFMO film. Between 350 - $450 \mathrm{~nm}$ (within the bandgap absorption of the BFMO but below the $\mathrm{Au} / \mathrm{Au} @ \mathrm{SiO}_{2}$ LSPR wavelengths), there is still wavelength-dependent enhancement in the IPCE spectra. However, PIRET between the Au NPs or $\mathrm{Au} @ \mathrm{SiO}_{2}$ NPs and the BFMO or hot electron injection from the bare Au NPs to the BFMO thin film are not possible at these wavelengths since the LSPR of the $\mathrm{Au} / \mathrm{Au} @ \mathrm{SiO}_{2} \mathrm{NPs}$ is not excited. To determine the source of the IPCE enhancement, UVVisible light absorption spectroscopy was performed for the BFMO-DNA-Au (Fig. 4.8) samples before and after incubation with $500 \mathrm{ppb} \mathrm{Hg}^{2+}$ and $\mathrm{Au}$ NPs. From Figures S4, there is an increase in light absorption between $350-450 \mathrm{~nm}$ for the BFMO-DNA-Au case. 


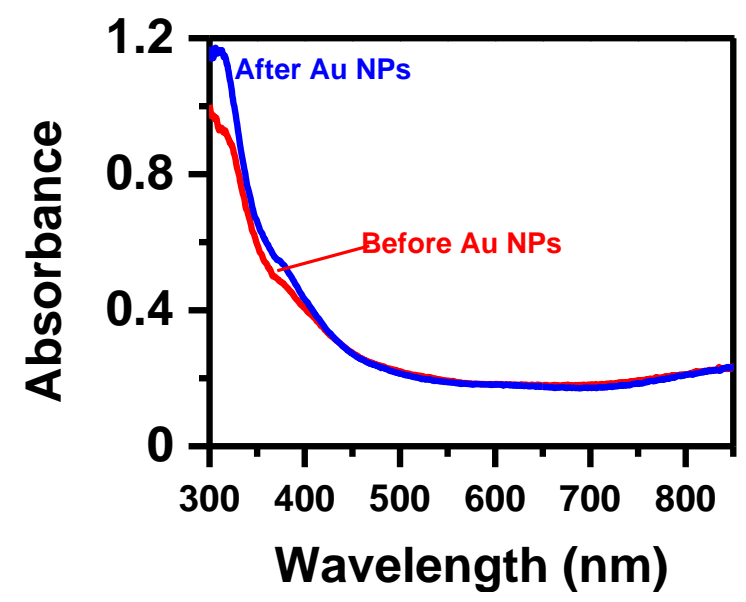

Figure 4.8: UV-Visible light absorption spectra of BFMO-DNA films before and after addition of

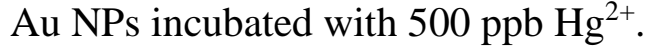

This means that at least part of the photocurrent enhancement is due to an optical absorption enhancement not directly related to the LSPR of the $\mathrm{Au}$ and $\mathrm{Au} @ \mathrm{SiO}_{2} \mathrm{NPs}$. However, the source of this optical absorption enhancement has not been fully explored. Given the wide spectral range of enhancement, Au NPs were used as the plasmonic nanoparticle for subsequent sensor tests for detection of $\mathrm{Hg}^{2+}$ in deionized water (Fig. 4.9).
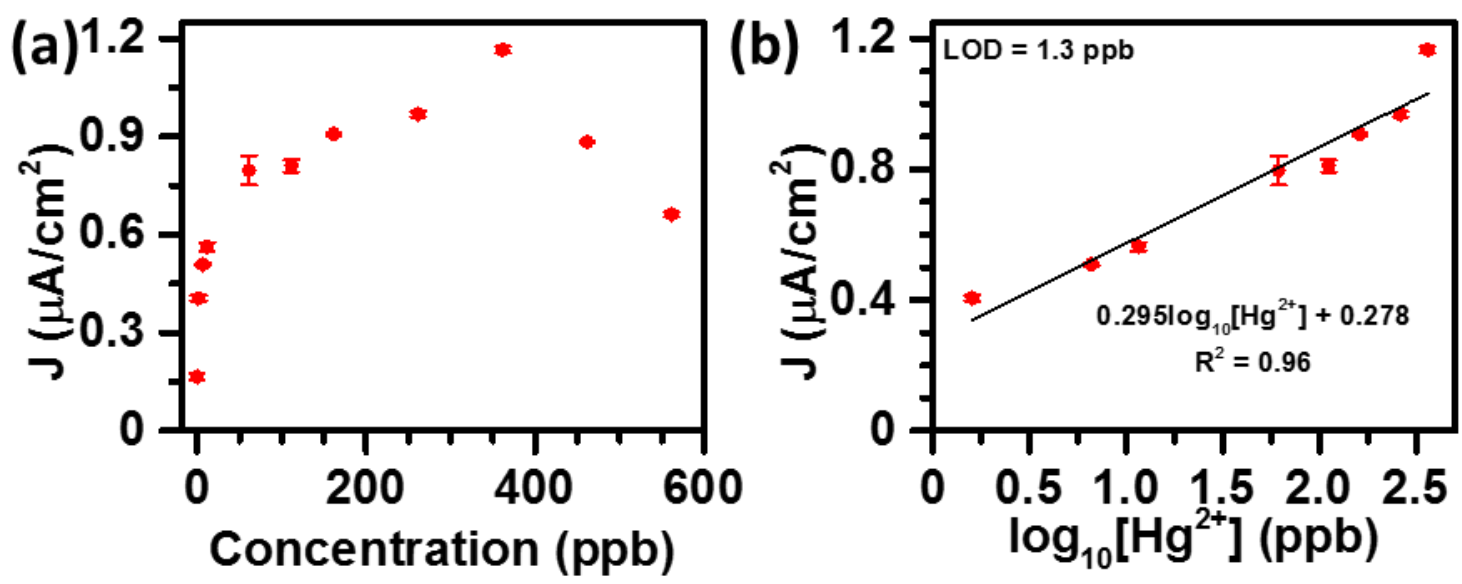

Figure 4.9: (a) Baseline subtracted photocurrent for BFMO-DNA-Au films as a function of incubated $\mathrm{Hg}^{2+}$ concentration; (b) Linear $(1.6-360 \mathrm{ppb})$ range of the photocurrent increase curve with linear fit.

From Figure 4.9(a), BFMO incubated with $\mathrm{Hg}^{2+}$ and $\mathrm{Au}$ NPs results in a photocurrent increase. The change in photocurrent increases linearly with respect to the logarithmically (base 10) with increasing $\mathrm{Hg}^{2+}$ concentration up to $360 \mathrm{ppb}$ followed by a significantly decrease at $\mathrm{Hg}^{2+}$ concentrations higher than $360 \mathrm{ppb}$. The decrease in photocurrent at higher $\mathrm{Hg}^{2+}$ concentrations is 
attributed to a combination of the Au NPs blocking light and surface reaction sites. The linear range for $\mathrm{Hg}^{2+}$ detection would be sufficient for the typical concentrations of $\mathrm{Hg}^{2+}$ in wastewater and starts just below the limit of $\mathrm{Hg}^{2+}$ in drinking water within the United States. ${ }^{[40-41]}$ This demonstrates the practical applications of the BFMO-DNA-Au conjugate photoelectrode for sensing of $\mathrm{Hg}^{2+}$. To further prove potential application of the BFMO-DNA-Au conjugate photoelectrode, the selectivity of the BFMO-DNA-Au photoelectrode for detection of $\mathrm{Hg}^{2+}$ was measured with and without the presence of potential interference cations $\left(\mathrm{Fe}^{3+}, \mathrm{Zn}^{2+}, \mathrm{Cu}^{2+}, \mathrm{As}^{3+}\right.$, $\mathrm{Cd}^{2+}$, and $\mathrm{Pb}^{2+}$ ) and shown in Figure 4.10.

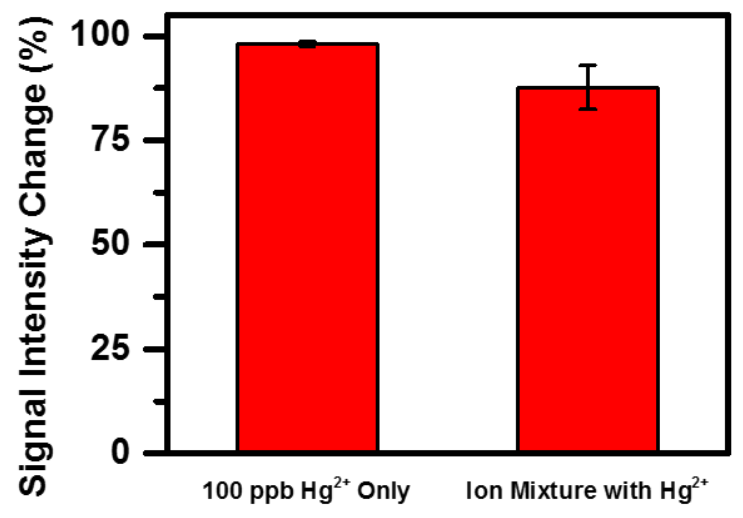

Figure 4.10: Signal intensity change of the photocurrent from the BFMO-DNA-Au towards $\mathrm{Hg}^{2+}$ only $\left(100 \mathrm{ppb} \mathrm{Hg}^{2+}\right)$ and the $100 \mathrm{ppb}$ interfering ion mixture (100 ppb Total of $\mathrm{Fe}^{3+}, \mathrm{Zn}^{2+}, \mathrm{Cu}^{2+}$, $\mathrm{As}^{3+}, \mathrm{Cd}^{2+}$, and $\mathrm{Pb}^{2+}$ ) with $100 \mathrm{ppb}$ of added $\mathrm{Hg}^{2+}$ in DI water.

Figure 4.10 shows a $98.1 \pm 0.6 \%$ increase in photocurrent versus baseline is obtained when incubating the BFMO film with $100 \mathrm{ppb} \mathrm{Hg}^{2+}$ and Au NPs. There is a $21.4 \pm 0.2 \%$ decrease in photocurrent upon incubation with a mixture of $100 \mathrm{ppb}$ combined of interfering ions without any added $\mathrm{Hg}^{2+}$. However, the photocurrent again increases by $87.5 \pm 3.2 \%$ versus the baseline photocurrent without $\mathrm{Hg}^{2+}$ and $\mathrm{Au}$ incubation, indicating that the interfering ions at low concentrations do not interfere significantly with the $\mathrm{Hg}^{2+}$ binding to the BFMO-DNA substrate. Overall, the concept of utilizing PIRET between plasmonic nanoparticles and a semiconductor film combined with the conjugation of plasmonic nanoparticles to the semiconductor to enhance the semiconductor's photocurrent has been demonstrated as a potential sensor working mechanism.

\subsection{Conclusions}

In this chapter, a comparison between hot electron injection and PIRET as photocurrent enhancement mechanisms is explored using plasmonic $\mathrm{Au}$ and $\mathrm{Au} @ \mathrm{SiO}_{2}$ nanoparticles in 
conjunction with a BFMO semiconductor photoanode using ssDNA strands with mismatched thymine bases and $\mathrm{Hg}^{2+}$ to facilitate DNA conjugation. Au NPs were found to enhance the BFMO's photocurrent through a combination of hot electron injection, PIRET, and Fermi-level equilibration, and an optical absorption enhancement mechanism at wavelengths where the Au NPs LSPR is not excited. Au@ $\mathrm{SiO}_{2}$ NPs were found to enhance the BFMO's photocurrent through PIRET and the previously mentioned optical absorption enhancement mechanism. A PEC sensor for detecting $\mathrm{Hg}^{2+}$ in water was demonstrated using the BFMO-DNA-Au conjugate photoanode due to the higher magnitude of enhancement across a wide wavelength spectrum versus the BFMO-DNA-Au@ $\mathrm{SiO}_{2}$ photoanode. The resulting BFMO-DNA-Au sensor has a limit of detection $(1.3 \mathrm{ppb})$ and linear range $(1.6-360 \mathrm{ppb})$ that is sufficient for detection of $\mathrm{Hg}^{2+}$ in common wastewater sources and drinking water. Further research will be focused on optimizing the effectiveness of the plasmonic energy transfer from the plasmonic metal to different semiconductors.

\subsection{Methods}

\subsubsection{Materials}

All chemicals and materials were used as received without further purification. (3aminopropyl)trimethoxysilane (97\%), ammonium hydroxide (28\% $\mathrm{NH}_{3}$ in $\mathrm{H}_{2} \mathrm{O}$ ), bismuth (III) nitrate pentahydrate (ACS, 98\%), copper (II) nitrate hemi(pentahydrate), hydrochloric acid (36\% w/w), hydrogen tetrachloroaurate (III) trihydrate (ACS, 99.99\%), iron (III) nitrate nonahydrate (98+\% metals basis), and trisodium citrate dihydrate (ACS, $99.0 \%$ min) were purchased from AlfaAesar. Acetone, citric acid, isopropyl alcohol, mercury (II) chloride, reagent alcohol, and sucrose were purchased from VWR International. Triethoxysilylpropyl succinic anhydride (TEPSA) was purchased from Gelest, Inc. 1-ethyl-3-(3-(dimethylamino)-propyl) carbodiimide (EDC), arsenic ICP/DCP standard solution, bovine serum albumin (BSA), cadmium (II) nitrate tetrahydrate, deoxyadenosine triphosphate (dATP), N-hydroxysuccinimide (NHS), lead (II) nitrate, phosphate buffered saline (PBS, $\mathrm{pH}=7.4$ ) tablets, sodium chloride, sodium dodecyl sulfate (SDS), sodium phosphate $\left(\mathrm{Na}_{3} \mathrm{PO}_{4} \cdot 12 \mathrm{H}_{2} \mathrm{O}\right)$, sodium silicate solution (reagent grade), and Tween 20 were purchased from Sigma-Aldrich. Zinc (II) nitrate hexahydrate was purchased from Strem Chemicals. Ammonium molybdate tetrahydrate $\left(\left(\mathrm{NH}_{4}\right)_{6} \mathrm{Mo}_{7} \mathrm{O}_{24} \cdot 4 \mathrm{H}_{2} \mathrm{O}\right)$ was purchased from Ward's Science. Fluorine-doped tin oxide (FTO) coated glass (TEC 15) was purchased from MTI Corporation.

DNA probes were purchased from Integrated DNA Technologies, Inc. (Coralville, IA) and have the following sequences:

Detection DNA for $\mathrm{SiO}_{2}$ Coated NPs (amine functionalized): 5'-/ $\mathrm{NH}_{2} / \mathrm{CAGTTTGAC-3}$ '

Detection DNA for Au NPs (Thiol Functionalized): 5'-/ThioMC6-D/AAAAAACAGTTTGAC-3' 
Capture DNA probe for BFMO (amine functionalized: 5'-/5AmMC6/GTCTTTCTG/ $\mathrm{NH}_{2} / 3^{\prime}$ '

\subsubsection{Characterization}

UV-Visible light absorption spectra were measured using a Shimadzu UV-2550 spectrometer with an integrating sphere (Shimadzu UV 2401/2) using $\mathrm{BaSO}_{4}$ as the optical "white" reference material. Field emission scanning electron microscopy (FESEM) was performed using JEOL JSM-7600 and Hitachi S-4700 FESEMs. TEM images were obtained using a JEOL JEM 2100F TEM.

\subsubsection{Gold Nanoparticles (Au NPs) Synthesis}

Gold nanoparticles (Au NPs) were synthesized using a traditional citrate reduction method $^{[42,43]}$. In a typical synthesis, $0.0197 \mathrm{~g}$ of hydrogen tetrachloroaurate (III) trihydrate $\left(\mathrm{HAuCl}_{4} \cdot 3 \mathrm{H}_{2} \mathrm{O}\right)$ was dissolved in $100 \mathrm{~mL}$ of DI water. This solution was then heated to boiling. Once the $\mathrm{HAuCl}_{4} \cdot 3 \mathrm{H}_{2} \mathrm{O}$ solution was boiling, $3 \mathrm{~mL}$ of a $1 \%(\mathrm{w} / \mathrm{v})$ trisodium citrate dihydrate in DI water solution was added to the $\mathrm{HAuCl}_{4} \cdot 3 \mathrm{H}_{2} \mathrm{O}$ solution where the color changed from light yellow to wine-red over a period of a few minutes. The $\mathrm{HAuCl}_{4} \cdot 3 \mathrm{H}_{2} \mathrm{O} /$ citrate solution was boiled continuously for another 30 minutes and then allowed to cool to room temperature naturally. The as-prepared Au NPs solution was stored for further use.

\subsubsection{Gold@Silicon Dioxide Core@Shell Nanoparticles (Au@SiO $\mathrm{NPs}_{2}$ Synthesis}

Silicon dioxide coating of the Au NPs was performed using a condensation-polymerization method $^{[44,45]}$. In a typical synthesis, $200 \mu \mathrm{L}$ of a $2 \mathrm{mM}$ (3-aminopropyl)trimethoxysilane aqueous solution was added to $20 \mathrm{~mL}$ of the as-prepared Au NPs solution and stirred for 30 minutes. Then, $1 \mathrm{~mL}$ of a $0.54 \mathrm{wt} \%$ sodium silicate solution was added dropwise under stirring to the Au NPs solution. The Au NPs solution was then stirred for another 10 minutes and then allowed to stand overnight. $10 \mathrm{~mL}$ of reagent alcohol was then added to the Au NPs solution. The Au NPs solution was stirred for 10 minutes and then allowed to stand overnight. Another $10 \mathrm{~mL}$ of reagent alcohol was then added to the Au NPs solution. The solution was again stirred for 10 minutes and then allowed to stand overnight. The resulting $\mathrm{Au} @ \mathrm{SiO}_{2} \mathrm{NPs}$ solution was then collected and washed using reagent alcohol at least 3 times via centrifugation. The $\mathrm{Au} @ \mathrm{SiO}_{2} \mathrm{NPs}$ were finally redispersed in reagent alcohol for further use. 


\subsection{5 $\mathrm{Bi}_{3} \mathrm{FeMo}_{2} \mathrm{O}_{12}$ (BFMO) Thin Film Synthesis}

$\mathrm{Bi}_{3} \mathrm{FeMo}_{2} \mathrm{O}_{12}$ (BFMO) thin films were synthesized using a modified "Pechini" complex precursor containing $\mathrm{Bi}(\mathrm{III}), \mathrm{Fe}(\mathrm{III})$, and $\mathrm{Mo}(\mathrm{VI})$ salts as metal sources. $2.91 \mathrm{~g}$ of bismuth (III) nitrate pentahydrate and $0.808 \mathrm{~g}$ of iron (III) nitrate were dissolved in $10 \mathrm{~mL}$ of ethylene glycol. Then, $0.706 \mathrm{~g}$ of ammonium molybdate tetrahydrate and $4.61 \mathrm{~g}$ of citric acid were added and dissolved in the precursor solution. The BFMO precursor solution was stirred overnight at room temperature before use.

FTO glass substrates were cleaned by alternating ultrasonication in reagent alcohol, 9\% w/w hydrochloric acid, acetone, and isopropyl alcohol for 20 minutes each followed by treatment under a radio frequency excited oxygen plasma for 90 seconds to ensure substrate hydrophilicity. BFMO deposition onto the cleaned FTO glass substrates was performed via spin coating of the BFMO precursor (4000 RPM, 100 seconds) followed by drying the films at $225^{\circ} \mathrm{C}$ for 15 minutes. The BFMO films were then placed into a muffle furnace and sintered at $650{ }^{\circ} \mathrm{C}$ for 1 hour $(1$ ${ }^{\circ} \mathrm{C} / \mathrm{min}$ ramp from $25^{\circ} \mathrm{C}$ to $600{ }^{\circ} \mathrm{C}$ with $10^{\circ} \mathrm{C} / \mathrm{min}$ ramp from $600{ }^{\circ} \mathrm{C}$ to $650{ }^{\circ} \mathrm{C}$; cooling to $25^{\circ} \mathrm{C}$ at $\left.10{ }^{\circ} \mathrm{C} / \mathrm{min}\right)$.

\subsubsection{Labeling $\mathrm{Ag} @ \mathrm{SiO}_{2}$ and $\mathrm{Au} @ \mathrm{SiO}_{2}$ with Amine Group Linked Detection DNA Probe}

Labeling of silicon dioxide coated nanoparticles was performed based on procedures in literature. ${ }^{[46]} 3.0 \mathrm{~mL}$ of $\mathrm{Ag} @ \mathrm{SiO}_{2}$ or $\mathrm{Au} @ \mathrm{SiO}_{2}$ nanoparticles were mixed with $20 \mu \mathrm{L}$ of TEPSA. The mixture solution was incubated overnight to achieve carboxyl group-terminated $\mathrm{Ag} @ \mathrm{SiO}_{2}$ nanoparticles. After washing with ethanol and D.I. water for several times, the resulting nanoparticles were re-suspended in $0.5 \mathrm{~mL}$ of the solution containing $50 \mathrm{mM}$ NHS and $200 \mathrm{mM}$ EDC. After incubation for $2 \mathrm{~h}$, the $\mathrm{COOH}$ group was activated. $50 \mu \mathrm{L}$ of $20 \mu \mathrm{M}$ ssDNA (amine group labeled detection DNA) solution was then added into the mixture. After overnight incubation, the solution was washed with a buffer solution (PBS containing $0.1 \%$ of BSA) for

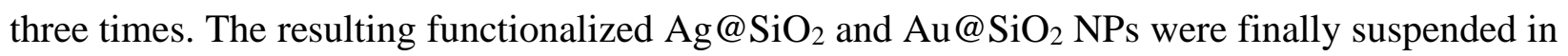
$200 \mu \mathrm{L}$ of eluent buffer $\left(20 \mathrm{nM}\right.$ of $\mathrm{Na}_{3} \mathrm{PO}_{4} \cdot 12 \mathrm{H}_{2} \mathrm{O}$ containing $5 \%$ BSA, $0.25 \%$ Tween 20 , and $10 \%$ sucrose) and stored at $4{ }^{\circ} \mathrm{C}$ for future use.

\subsubsection{Labeling Au with Thiol-Group Linked Detection DNA Probe}

The Au NPs/detection DNA conjugate was prepared according to the reported methods ${ }^{[47]}$. dATP was added into $1 \mathrm{~mL}$ of concentrated Au NPs solution (final concentration of dATP is 7.05 $\mu \mathrm{M})$. The mixture was incubated at room temperature for 20 minutes. $15 \mu \mathrm{L}$ of $1 \%$ of SDS was slowly added into the mixture and incubated using a shaker for 10 minutes. $50 \mu \mathrm{L}$ of $2 \mathrm{M} \mathrm{NaCl}$ 
aqueous solution was dropped into the mixture at a rate of $2 \mu \mathrm{L} / 2 \mathrm{~min}$. Then, $0.25 \mathrm{OD}$ of thiolated detection DNA was added and the mixture was incubated for 3 hours in oven at $60^{\circ} \mathrm{C}$. After the incubation, the mixture was centrifuged at $12000 \mathrm{rpm}$ for 15 minutes. The supernatant was discarded. The Au NPs were then washed with $1 \mathrm{~mL}$ of PBS for 3 times. The resulting red sediments were re-suspended in $500 \mu \mathrm{L}$ of eluent buffer $\left(20 \mathrm{nM}\right.$ of $\mathrm{Na}_{3} \mathrm{PO}_{4} \cdot 12 \mathrm{H}_{2} \mathrm{O}$ containing $5 \%$ BSA, $0.25 \%$ Tween 20 and $10 \%$ sucrose).

\subsubsection{Labeling Amine Group Linked Capture DNA Probe on BFMO Films}

The BFMO films were labelled with capture DNA according to reported methods ${ }^{[47]}$. The BFMO films on FTO glass were first cleaned by successive immersion in ethanol and DI water each for $10 \mathrm{~min}$. The cleaned BFMO films were incubated overnight in an ethanolic solution containing $0.5 \%$ TEPSA and then washed with ethanol to remove free TEPSA. The resulting TEPSA-modified BFMO films were activated by immersion in a PBS solution containing $50 \mathrm{mM}$ NHS and $200 \mathrm{mM}$ EDC. After being washed with PBS solution, chips were incubated overnight in PBS solution containing $20 \mu \mathrm{M}$ of ssDNA (amine group labeled capture DNA), followed by rigorously washing with PBS solution to remove free capture DNA and kept in a humid chamber prior to assay.

\subsubsection{Photoelectrochemical (PEC) Testing}

All photoelectrochemical (PEC) testing was performed using a three-electrode cell configuration in a $0.1 \mathrm{M}$ phosphate buffered saline (PBS) $(\mathrm{pH}=7.4)$ aqueous electrolyte. BFMODNA thin films with and without plasmonic nanoparticles functionalization were used as the working electrodes. An $\mathrm{Ag} \mid \mathrm{AgCl}$ electrode (Sat. KCl; $\mathrm{E}^{\mathrm{O}}=+0.197 \mathrm{~V}$ vs. $\mathrm{NHE}$ ) and a platinum mesh were used as the reference and counter electrodes, respectively. All PEC measurements were made using a Gamry Reference 3000 potentiostat/galvanostat/ZRA instrument.

$\mathrm{J}-\mathrm{V}$ curves were recorded using simulated sunlight from a $300 \mathrm{~W}$ Xe arc lamp with an AM1.5G filter calibrated to $100 \mathrm{~mW} / \mathrm{cm}^{2}$ using a thermopile sensor (Newport 818P) as the light source. Wavelength-dependent incident photon-to-current efficiency (IPCE) measurements were performed using light from the $300 \mathrm{~W}$ Xe arc lamp channeled through a monochromator (Oriel Cornerstone $^{\mathrm{TM}} 1301 / 8 \mathrm{~m}$ ) as the light source. Wavelength-dependent optical power measurements were performed using a Newport 71675 silicon photodiode detector. The IPCE for a given light wavelength was calculated using Equation 1: ${ }^{[48]}$

$$
I P C E=\frac{1240 \mathrm{~J}}{\lambda \cdot P}
$$


where $J$ (in $\left.\mathrm{mA} / \mathrm{cm}^{2}\right)$ is the photocurrent measured under a given light wavelength $(\lambda$ in $\mathrm{nm})$ and $P$ is the optical power density (in $\mathrm{mW} / \mathrm{cm}^{2}$ ) of the incident light at a given light wavelength.

Mott-Schottky (M-S) plots were obtained at $f=5000 \mathrm{~Hz}$ with an applied AC bias of $10 \mathrm{mV}$ RMS. The obtained electrochemical impedance spectra were used to calculate the space charge capacitance using Equation 2: ${ }^{[4]}$

$$
Z_{i m g}=\frac{1}{2 \pi f C}
$$

where $Z_{i m g}$ is the imaginary component of the measured electrochemical impedance, $f$ is the frequency of the applied AC bias, and $C$ is the space charge capacitance of the sample.

\subsubsection{Mercury (II) Ion $\left(\mathrm{Hg}^{2+}\right)$ PEC Sensor Testing}

Initially, the baseline PEC performance (J-V curves, M-S, and IPCE) of BFMO films labeled with only the capture DNA was tested. Then, the BFMO films are conjugated with mercury (II) ions $\left(\mathrm{Hg}^{2+}\right)$ and plasmonic nanoparticles in a two-step process. $50 \mu \mathrm{L}$ of target solution containing various mercury (II) ion concentrations $(0-500 \mathrm{ppb})$ were dropped onto the detection area of the BFMO chip. After incubation for $30 \mathrm{~min}$, the BFMO film was vigorously rinsed with PBS to remove unbound $\mathrm{Hg}^{2+}$ ions. Then, $50 \mu \mathrm{L}$ of the synthesized detection DNA probe linked plasmonic nanoparticles conjugates were dropped onto the detection area and incubated for 30 min, followed by rinsing with PBS to remove free conjugates. The resulting BFMO-DNAplasmonic nanoparticle sandwich films was subject to the PEC measurements.

The sensitivity and selectivity of the BFMO-DNA-Au photoelectrodes towards $\mathrm{Hg}^{2+}$ was measured using simulated sunlight from $300 \mathrm{~W}$ Xe lamp calibrated to $100 \mathrm{~mW} / \mathrm{cm}^{2}$ The limit of detection of the BFMO-DNA-Au was determined using Equation 3: ${ }^{[50]}$

$$
L O D=10^{\frac{3 \cdot(S D)}{\text { Slope }}}
$$

where $L O D$ is the limit of detection for $\mathrm{Hg}^{2+}, \mathrm{SD}$ is the standard deviation of the photocurrent during measurement of a blank sample $\left(0 \mathrm{ppb}\right.$ of $\left.\mathrm{Hg}^{2+}\right)$, and Slope is the slope of the linear region of the photocurrent as a function of added $\mathrm{Hg}^{2+}$ concentration.

\subsection{References}

[1] Lee, R. The Outlook for Population Growth. Science 2011, 333, 569-573

[2] Zhao, W.-W.; Xu, J.-J.; Chen, H.-Y. Photoelectrochemical bioanalysis: the state of the art. Chem. Soc. Rev. 2015, 44, 729-741 
[3] Peng, M.; Guan, G.; Deng, H.; Han, B.; Tian, C.; Zhuang, J.; Xu, Y.; Liu, W.; Lin, Z. PCN224/rGO nanocomposite based photoelectrochemical sensor with intrinsic recognition ability for efficient p-arsanilic acid detection. Envrion. Sci.: Nano 2019, 6, 207-215.

[4] Zhang, B.; Guo, L.-H. Highly sensitive and selective photoelectrochemical DNA sensor for the detection of $\mathrm{Hg}^{2+}$ in aqueous solutions. Biosens. Bioelectron. 2012, 37, 112-115.

[5] Li, H.; Li, J.; Yang, Z.; Xu, Q.; Hu, X. A novel photoelectrochemical sensor for the organophosphorus pesticide dichlofenthion based on nanometer-sized titania coupled with a screen-printed electrode. Anal. Chem. 2011, 83, 5290-5295.

[6] Maier, S. A.; Atwater, H. A. Plasmonics: Localization and guiding of electromagnetic energy in metal/dielectric structures. J. Appl. Phys. 2005, 98, 011101.

[7] Wu, N. Plasmonic metal-semiconductor photocatalysts and photoelectrochemical cells: a review. Nanoscale 2018, 10, 2679-2696.

[8] Stewart, M. E.; Anderton, C. R.; Thompson, L. B.; Maria, J.; Gray, S. K.; Rogers, J. A.; Nuzzo, R. G. Nanostructured plasmonic sensors. Chem. Rev. 2008, 108, 494-521.

[9] Schuller, J. A.; Barnard, E. S.; Cai, W.; Jun, Y. C.; White, J. S.; Brongersma, M. L. Plasmonics for extreme light concentration and manipulation. Nat. Mater. 2010, 9, 193205.

[10] Atwater, H. A.; Polman, A. Plasmonics for improved photovoltaic devices. Nat. Mater. 2010, 9, 205-213.

[11] Li, Y.; Cheng, J.; Liu, Y.; Liu, P.; Cao, W.; He, T.; Chen, R.; Tang, Z. Manipulation of surface plasmon resonance in sub-stoichiometry molybdenum oxide nanodots through charge carrier control technique. J. Phys. Chem. C 2017, 121, 5208-5214.

[12] Lou, Z.; Zhu, M.; Yang, X.; Zhang, Y.; Whangbo, M.-H.; Li, B.; Huang, B. Continual injection of photoinduced electrons stabilizing surface plasmon resonance of nonelemental-metal plasmonic photocatalyst $\mathrm{CdS} / \mathrm{WO}_{3-\mathrm{x}}$ for efficient hydrogen generation. Appl. Catal. B-Environ. 2018, 226, 10-15.

[13] Kriegel, I.; Jiang, C.; Rodriguez-Fernandez, J.; Schaller, R. D.; Talapin, D. V.; da Como, E.; Feldmann, J. Tuning the excitonic and plasmonic properties of copper chalcogenide nanocrystals. J. Am. Chem. Soc. 2012, 134, 1583-1590.

[14] Catchpole, K. R.; Polman, A. Design principles for particle plasmon enhanced solar cells. Appl. Phys. Lett. 2008, 93, 191113. 
[15] Seh, Z. W.; Liu, S.; Low, M.; Zhang, S.-Y.; Liu, Z.; Mlayah, A.; Han, M.-Y. Janus Au-TiO 2 photocatalysts with strong localization of plasmonic near-fields for efficient visible-light hydrogen generation. Adv. Mater. 2012, 24, 2310-2314.

[16] Cushing, S. K.; Li, J.; Meng, F.; Senty, T. R.; Suri, S.; Zhi, M.; Li, M.; Bristow, A. D.; Wu, N. Photocatalytic activity enhanced by plasmonic resonant energy transfer from metal to semiconductor. J. Am. Chem. Soc. 2012, 134, 15033-15041.

[17] Li, J.; Cushing, S. K.; Meng, F.; Senty, T. R.; Bristow, A. D.; Wu, N. Plasmon-induced resonance energy transfer for solar energy conversion. Nat. Photon. 2015, 9, 601-608.

[18] Meng, F.; Cushing, S. K.; Li, J.; Hao, S.; Wu, N. Enhancement of solar hydrogen generation by synergistic interaction of $\mathrm{La}_{2} \mathrm{Ti}_{2} \mathrm{O}_{7}$ photocatalyst with plasmonic gold nanoparticles and reduced graphene oxide nanosheets. ACS Catal. 2015, 5, 1949-1955.

[19] Li, G.; Cherqui, C.; Bigelow, N. W.; Duscher, G.; Straney, P. J.; Millstone, J. E.; Masiello, D. J.; Camden, J. P. Spatially mapping energy transfer from single plasmonic particles to semiconductor substrates via STEM/EELS. Nano Lett. 2015, 15, 3465-3471.

[20] Tian, Y.; Tatsuma, T. Plasmon-induced photoelectrochemistry at metal nanoparticles supported on nanoporous $\mathrm{TiO}_{2}$. Chem. Commun. 2004, 1810-1811.

[21] Clavero, C. Plasmon-induced hot-electron generation at nanoparticle/metal-oxide interfaces for photovoltaic and photocatalytic devices. Nat. Photon. 2014, 8, 95-103.

[22] Sousa-Castillo, A.; Comesaña-Hermo, M.; Rodríguez-González, B.; Pérez-Lorenzo, M.; Wang, Z.; Kong, X.-T.; Govorov, A. O.; Correa-Duarte, M. A. Boosting hot electrondriven photocatalysis through anisotropic plasmonic nanoparticles with hot spots in $\mathrm{Au}-$ $\mathrm{TiO}_{2}$ nanoarchitectures. J. Phys. Chem. C 2016, 120, 11690-11699.

[23] Sundararaman, R.; Narang, P.; Jermyn, A. S.; Goddard III, W. A.; Atwater, H. A. Theoretical predictions for hot-carrier generation from surface plasmon decay. Nat. Commun. 2014, 5, 5788.

[24] DuChene, J. S.; Tagliabue, G.; Welch, A. J.; Cheng, W.-H.; Atwater, H. A. Hot hole collection and photoelectrochemical $\mathrm{CO}_{2}$ reduction with plasmonic $\mathrm{Au} / \mathrm{p}-\mathrm{GaN}$ photocathodes. Nano Lett. 2018, 18, 2545-2550.

[25] Da, P.; Li, W.; Lin, X.; Wang, Y.; Tang, J.; Zheng, G. Surface plasmon resonance enhanced real-time photoelectrochemical protein sensing by gold nanoparticle-decorated $\mathrm{TiO}_{2}$ nanowires. Anal. Chem. 2014, 86, 6633-6639.

[26] Xin, Y.; Li, Z.; Zhang, Z. Photoelectrochemical aptasensor for the sensitive and selective detection of kanamycin based on Au nanoparticle functionalized self-doped $\mathrm{TiO}_{2}$ nanotube arrays. Chem. Commun. 2015, 51, 15498-15501. 
[27] Shu, J.; Qiu, Z.; Lv, S.; Zhang, K.; Tang, D. Plasmonic enhancement coupling with defectengineered $\mathrm{TiO}_{2-\mathrm{x}}$ : A mode for sensitive photoelectrochemical biosensing. Anal. Chem. 2018, 90, 2425-2429.

[28] Qiao, Y.; Li, J.; Li, H.; Fang, H.; Fan, D.; Wang, W. A label-free photoelectrochemical aptasensor for bisphenol A based on surface plasmon resonance of gold nanoparticlesensitized ZnO nanopencils. Biosens. Bioelectron. 2016, 86, 315-320.

[29] Shi, Y.; Zhang, Q., Zhai, T.-T.; Zhou, Y.; Yang, D.-R.; Wang, F.-B.; Xia, X.-H. Localized surface plasmon resonance enhanced label-free photoelectrochemical immunoassay by $\mathrm{Au}-\mathrm{MoS}_{2}$ nanohybrid. Electrochimica Acta 2018, 271, 361-369.

[30] Zhu, Y.-C.; Zhang, N.; Ruan, Y.-F.; Zhao, W.-W.; Xu, J.-J.; Chen, H.-Y. Alkaline phosphatase tagged antibodies on gold nanoparticles/ $/ \mathrm{TiO}_{2}$ nanotubes electrode: A plasmonic strategy for label-free and amplified photoelectrochemical immunoassay. Anal. Chem. 2016, 88, 5626-5630.

[31] Zhao, W.-W.; Yu, P.-P., Shan, Y.; Wang, J.; Xu, J.-J.; Chen, H.-Y. Exciton-plasmon interactions between $\mathrm{CdS}$ quantum dots and $\mathrm{Ag}$ nanoparticles in photoelectrochemical system and its biosensing application. Anal. Chem. 2012, 84, 5892-5897.

[32] Dong, Y.-X.; Cao, J.-T.; Wang, B.; Ma, S.-H.; Liu, Y.-M. Exciton-plasmon interactions between $\mathrm{CdS} @ \mathrm{~g}-\mathrm{C}_{3} \mathrm{~N}_{4}$ heterojunction and $\mathrm{Au} @ \mathrm{Ag}$ nanoparticles coupled with DNAasetriggered signal amplification: Toward highly sensitive photoelectrochemical bioanalysis of microRNA. ACS Sustainable Chem. Eng. 2017, 5, 10840-10848.

[33] Liu, S.; Cao, H.; Wang, X.; Tu, W.; Dai, Z. Green light excited ultrasensitive photoelectrochemical biosensing for microRNA at a low applied potential based on the dual role of $\mathrm{Au}$ NPs in $\mathrm{TiO}_{2}$ nanorods/Au NPs composites. Nanoscale 2018, 10, 1647416478.

[34] Leenheer, A. J.; Narang, P.; Lewis, N. S.; Atwater, H. A. Solar energy conversion via hot electron internal photoemission in metallic nanostructures: efficiency estimates. J. Appl. Phys. 2014, 115, 134301.

[35] Cushing, S. K.; Bristow, A. D.; Wu, N. Theoretical maximum efficiency of solar energy conversion in plasmonic metal-semiconductor heterojunctions. Phys. Chem. Chem. Phys. 2015, 17, 30013-30022.

[36] Liu, B.; Yasin, A. S.; Musho, T.; Bright, J.; Tang, H.; Huang, L.; Wu, N. Visible-light bismuth iron molybdate photocatalyst for artificial nitrogen fixation. J. Electrochem. Soc. 2019, 166, H3091-H3096. 
[37] Cushing, S. K.; Chen, C.-J.; Dong, C. L.; Kong, X.-T.; Govorov, A. O.; Liu, R.-S.; Wu, N. Tunable nonthermal distribution of hot electrons in a semiconductor injected from a plasmonic gold nanostructure. ACS Nano 2018, 12, 7117-7126.

[38] Subramanian, V.; Wolf, E. E.; Kamat, P. V. Catalysis with $\mathrm{TiO}_{2}$ /gold nanocomposites. effect of metal particle size on the Fermi level equilibration. J. Am. Chem. Soc. 2004, 126, 4943-4950.

[39] Li. J.; Cushing, S. K.; Chu, D.; Zheng, P.; Bright, J.; Castle, C.; Manivannan, A.; Wu, N. Distinguishing surface effects of gold nanoparticles from plasmonic effect on photoelectrochemical water splitting by hematite. J. Mater. Res. 2016, 31, 1608-1615.

[40] Mercury Pollutant Minimization Program Guidance; U.S. Environmental Protection Agency Region 5 NPDES Programs Branch: Chicago, 2004.

[41] 2018 Edition of the Drinking Water Standards and Health Advisories; EPA 822-F-18-001; U.S. Environmental Protection Agency: Washington D.C., 2018.

[42] Frens, G. Controlled nucleation for the regulation of the particle size in monodisperse gold suspensions. Nat. Phys. Sci. 1973, 241, 20-22.

[43] Kimling, J.; Maier, M.; Okenve, B.; Kotaidis, V.; Ballot, H.; Plech, A. Turkevich method for gold nanoparticle synthesis revisited. J. Phys. Chem. B 2006, 110, 15700-15707.

[44] Liz-Marzán, L. M.; Giersig, M.; Mulvaney, P. Synthesis of nanosized gold- silica coreshell particles. Langmuir 1996, 12, 4329-4335.

[45] Li, M.; Cushing, S. K.; Zhang, J.; Lankford, J.; Aguilar, Z. P.; Ma, D.; Wu, N. Shapedependent surface-enhanced Raman scattering in gold-Raman-probe-silica sandwiched nanoparticles for biocompatible applications. Nanotechnology 2012, 23, 115501.

[46] Gao, X.; Xu, H.; Baloda, M.; Gurung, A. S.; Xu, L.-P.; Wang, T.; Zhang, X.; Liu, G. Visual detection of microRNA with lateral flow nucleic acid biosensor. Biosens. Bioelectron. 2014, 54, 578-584.

[47] Zheng, P.; Li, M.; Jurevic, R.; Cushing, S. K.; Liu, Y.; Wu, N. A gold nanohole array based surface-enhanced Raman scattering biosensor for detection of silver (I) and mercury (II) in human saliva. Nanoscale 2015, 7, 11005-11012.

[48] Bak, T.; Nowotny, J.; Rekas, M.; Sorrell, C. C. Photo-electrochemical hydrogen generation from water using solar energy. Materials-related aspects. Int. J. Hydrogen Energy 2002, 27, 991-1022.

[49] Bott, A. W. Electrochemistry of semiconductors. Current Separations 1998, 17, 87-91.

[50] Long, G. L.; Winefordner, J. D. Limit of detection. A closer look at the IUPAC definition. Anal. Chem. 1983, 55, 712-724. 


\section{Chapter 5: Semiconductor Photoelectrochemical (PEC) Immunosensor Utilizing Plasmonic Energy Transfer for Immunoglobin G (IgG) Detection}

\subsection{Introduction}

As the human population continues to increase across the world, there is a need for new and improved strategies for ensuring the health of the population. One of the major concerns for ensuring the health of humanity is the improved detection and diagnosis of human illnesses. As rapid detection and diagnosis of illness significantly improves the prognosis for patient recovery, new biosensors must be developed to diagnose illness.

In recent years, significant focus has been spent on designing immunosensors, a type of biosensor that utilize antibody-antigen reactions for detection of proteins biomarkers that may be part of the human body's immune system response to illness. ${ }^{[1-3]}$ These protein biomarkers range from biomarkers for infectious disease agents such as bacteria and viruses to other proteins released as the result of trauma or non-infectious illness in the human body. The main appeal of immunosensors is the specificity and stability of the antibody-antigen binding events used to capture and detect desired analytes. As with other types of biosensors, immunosensors can utilize a variety of signal transduction mechanisms ranging from colorimetry ${ }^{[4-5]}$, fluorescence ${ }^{[6,7]}$, electrochemistry ${ }^{[1,2]}$, and surface enhanced Raman scattering (SERS) ${ }^{[8]}$.

Photoelectrochemical (PEC) immunosensors have been previously built using internally photoexcited electrical current from within a semiconductor photoelectrode to drive electrochemical reactions at the photoelectrode surface. These PEC immunosensors utilize different methods for modulating photocurrent, photovoltage, or charge transfer resistance in the presence of analytes as the sensing actuation mechanism. ${ }^{[3,9-23]}$ There are several advantages to PEC-based immunosensors versus other immunosensor techniques due to inexpensive equipment needed (light source and potentiostat) and relatively simple operation versus other techniques like SERS or commercially available colorimetric immunosensors like the enzyme-linked immunosorbent assay (ELISA) kits. ${ }^{[24,25]}$

Some PEC immunosensors incorporate nanoparticles made of plasmonic materials such as $\mathrm{Au}$ or $\mathrm{Ag}$ as an active component of the PEC immunosensor design. The appeal of plasmonic nanoparticles is the strong light absorption and intense localized electromagnetic fields around the plasmonic nanoparticles from their localized surface plasmon resonance (LSPR). While plasmonic nanoparticles are commonly used in colorimetric, fluorescence, or SERS-based biosensors, PEC immunosensors utilize plasmonic nanoparticles modulate photocurrent through Förster resonant energy transfer (FRET) from semiconductor photoelectrode to conjugated plasmonic nanoparticles $^{[22,23]}$ or changes to hot electron injection processes from already decorated plasmonic nanoparticles to the supporting semiconductor photoelectrode ${ }^{[19-21,26-28]}$. However, to 
the authors' knowledge, there are currently no immunosensors that utilize plasmon-induced resonant energy transfer (PIRET) from conjugated plasmonic nanoparticles to the semiconductor photoelectrode. PIRET is a plasmonic energy transfer process that utilizes non-radiative dipoledipole interactions to transfer energy stored in the localized surface plasmon resonance (LSPR) from plasmonic nanoparticles to nearby semiconductors. PIRET is a more efficient plasmonic energy transfer process than hot electron injection with as much as $30 \%$ of the harvested light energy converted into useful photoexcited carriers within the semiconductor photoelectrode versus a theoretical maximum hot carrier injection efficiency of $10 \% .{ }^{[29-31]}$ In addition, the nature of PIRET allows for plasmonic energy to be transferred across short distances even if the plasmonic nanoparticles are not contact with the semiconductor. While FRET and PIRET are both resonant energy transfer processes that rely on dipole-dipole interactions over similar distances, there are similar advantages between FRET and PIRET. However, the direction of energy transfer differs between those processes. Energy transfer from the semiconductor into the plasmonic nanoparticles by FRET decreases the semiconductor's photocurrent. However, PIRET from plasmonic nanoparticles to the semiconductor increases the semiconductor's photocurrent.

In this chapter, a PEC immunosensor utilizing PIRET between plasmonic gold nanoparticles ( $\mathrm{Au}$ NPs) and $\mathrm{a}_{3} \mathrm{Bi}_{3} \mathrm{FeMo}_{2} \mathrm{O}_{12}$ (BFMO) semiconductor thin film for detection of human immunoglobulin G (IGG) in buffer is demonstrated. Human IGG is chosen as the analyte due to it being a well-studied protein biomarker within the human body that allows for comparison of sensor performance across literature Au NPs are controllably linked to the BFMO thin film via anti-human IGG antibody-IGG antigen reactions. PIRET from the conjugated Au NPs results in an increased photocurrent that is proportional to the amount of conjugated Au NPs. While the resulting PIRET sensor shows some sensitivity towards IGG detection, the large separation distance between the Au NPs and BFMO film and weak dipole strength of the BFMO film necessitates further refinement of the PIRET sensor design.

\subsection{Results}

For this PIRET based PEC immunosensor requires multiple components in order to function. The first component is a semiconductor capable of driving PEC reactions. The semiconductor must have an optical absorbance that can overlap with the LSPR of plasmonic nanoparticles in order for PIRET to be possible. ${ }^{[30,31]}$ In addition, the semiconductor should also be thin enough such that light absorption rather than charge carrier transport within the semiconductor is limiting factor for photocurrent generation. For the semiconductor, $\mathrm{Bi}_{3} \mathrm{FeMo}_{2} \mathrm{O}_{12}$ (BFMO) was chosen due to its bandgap (2.25 eV) which overlaps with the LSPR of plasmonic Au NPs and its relative PEC stability. ${ }^{[32,33]}$ Figure 5.1 shows SEM images of synthesized BFMO thin films. From the cross-section and top-view SEM images in Figures 5.1a and 5.1b respectively, the synthesized BFMO thin films are between $150-200 \mathrm{~nm}$ in thickness and polycrystalline in nature with crystallites $50-200 \mathrm{~nm}$ in dimension. 


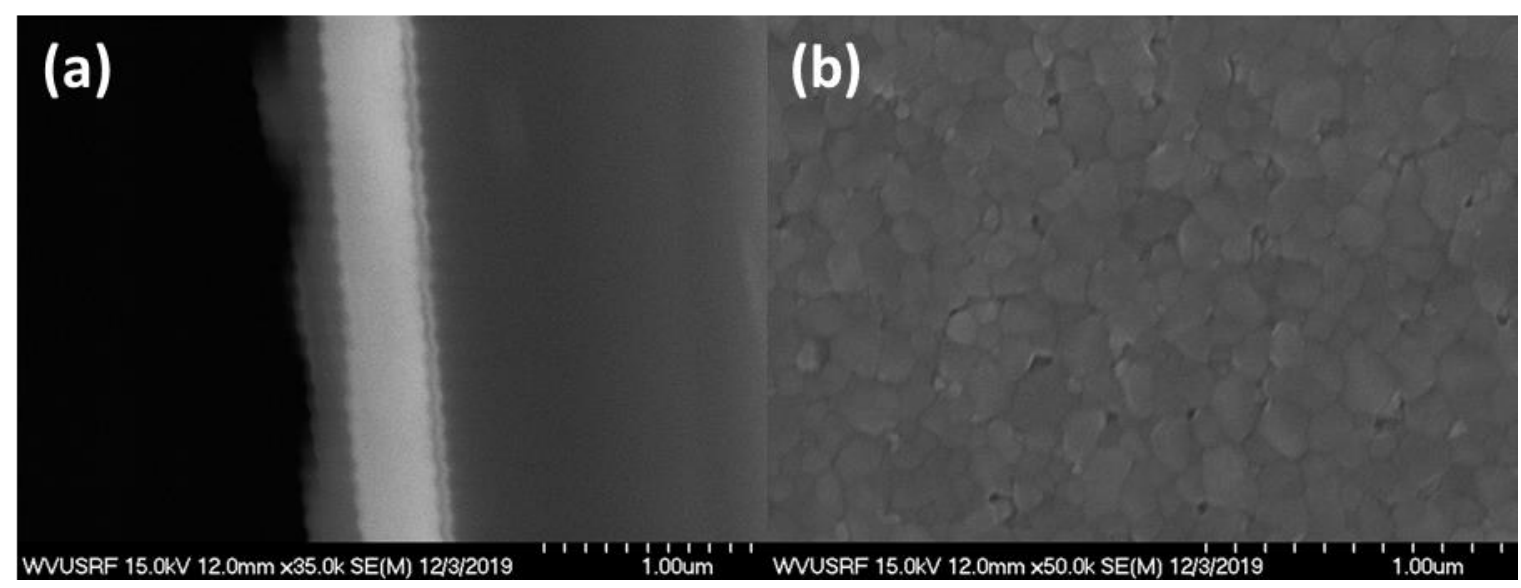

Figure 5.1: (a) Cross-Section and (b) Top view SEM images of $\mathrm{Bi}_{3} \mathrm{FeMo}_{2} \mathrm{O}_{12}$ (BFMO) thin film substrates.

To verify the successful synthesis of pure phase $\mathrm{Bi}_{3} \mathrm{FeMo}_{2} \mathrm{O}_{12}$ thin films, an X-ray diffraction spectrum (Figure 5.2) was collected of the BFMO thin films on FTO glass.

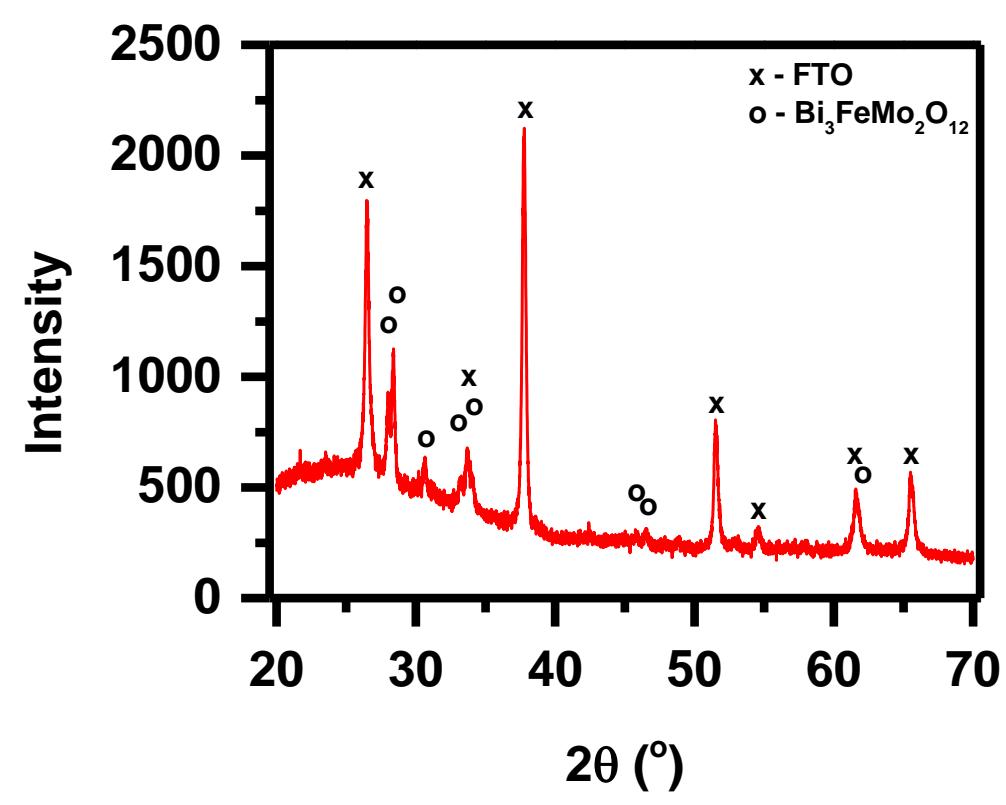

Figure 5.2: X-ray Diffraction (XRD) spectrum for $\mathrm{Bi}_{3} \mathrm{FeMo}_{2} \mathrm{O}_{12}(\mathrm{BFMO})$ film on fluorine doped tin oxide (FTO) coated glass substrate.

From Figure 5.2, there are two distinct sets of peaks detected. The first peak set characterized by major peaks at $26.5^{\circ}, 37.8^{\circ}$, and $51.8^{\circ}$ matches reference data for tin oxide $\left(\mathrm{SnO}_{2}\right.$; ICSD 98-003-9177) consistent with the conductive FTO layer on the FTO glass substrate. The second peak set characterized by major peaks at $28.0^{\circ}, 28.4^{\circ}, 30.6^{\circ}, 33.2^{\circ}$, and $34.0^{\circ}$ are consistent with monoclinic BFMO ( $\mathrm{Bi}_{3} \mathrm{FeMo}_{2} \mathrm{O}_{12}$; ICSD 98-000-0045). The lower intensity of the BFMO peaks relative to the FTO peaks is reasonable given the differences in thickness between the BFMO 
and FTO layers. No peaks corresponding to secondary impurity phases were detected within the spectrum in Figure 5.2, indicating the BFMO thin films are pure in phase.

After synthesizing pure phase BFMO thin films, in order to form an immunoassay, the BFMO are labeled with human immunoglobulin G (IGG) capture antibodies in accordance with the schematic in Figure 5.3. Liquid sample containing human IGG is deposited onto an anti-human IGG capture antibody labeled BFMO film. Human IGG within the deposited sample conjugates with the labeled human IGG capture antibodies on the surface with the number of conjugated antibodies directly proportional to the amount of human IGG contained within the deposited sample (Figure 5.3a). Subsequently, anti-human IGG capture antibody labeled Au NPs are added after the human IGG conjugation (Figure 5.3b). The antibody labeled Au NPs should only specifically bind to the antibodies on the BFMO that have already conjugated with IGG with sufficient washing to remove non-specifically bound Au NPs. To confirm successful labeling of the anti-human IGG capture antibodies onto the surface of the synthesized BFMO thin films, Fourier transform infrared (FTIR) spectroscopy (Figure 5.4) and X-ray photoelectron spectroscopy (XPS) (Figure 5.5) were performed on anti-human IGG antibody labeled BFMO thin films. 


\section{(a) Human Immunoglobulin G (IGG) Containing Sample Added to BFMO-Antibody (Ab) Film}

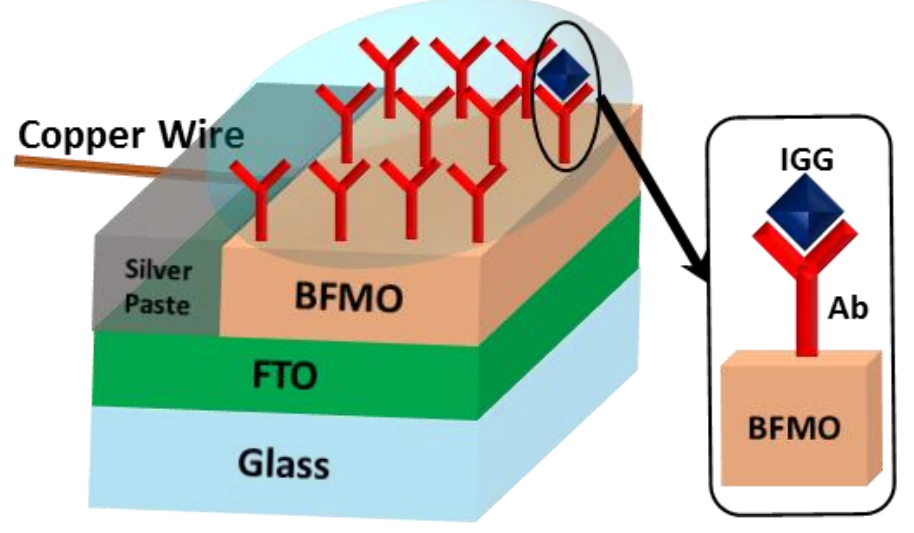

\section{(b) Antibody Functionalized Au NPs Conjugated with BFMO-Antibody-IGG Film}

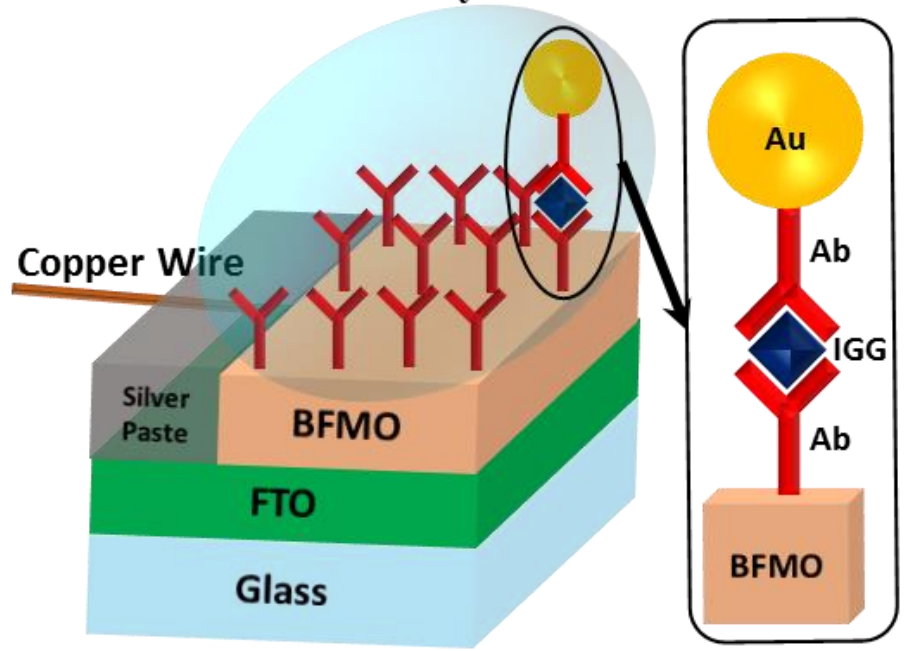

Figure 5.3: Schematic for conjugation of gold nanoparticles ( $\mathrm{Au} N \mathrm{NPs}$ ) with $\mathrm{Bi}_{3} \mathrm{FeMo}_{2} \mathrm{O}_{12}$ (BFMO) film for use as photoelectrochemical sensor for human immunoglobulin G (IGG) detection; (a) Human IGG containing sample incubated with anti-human IGG antibody (Ab) functionalized BFMO film (BFMO-Ab); (b) anti-human IGG antibody functionalized Au NPs added to BFMOAb-IGG film with Au NPs to bind with already conjugated human IGG. 


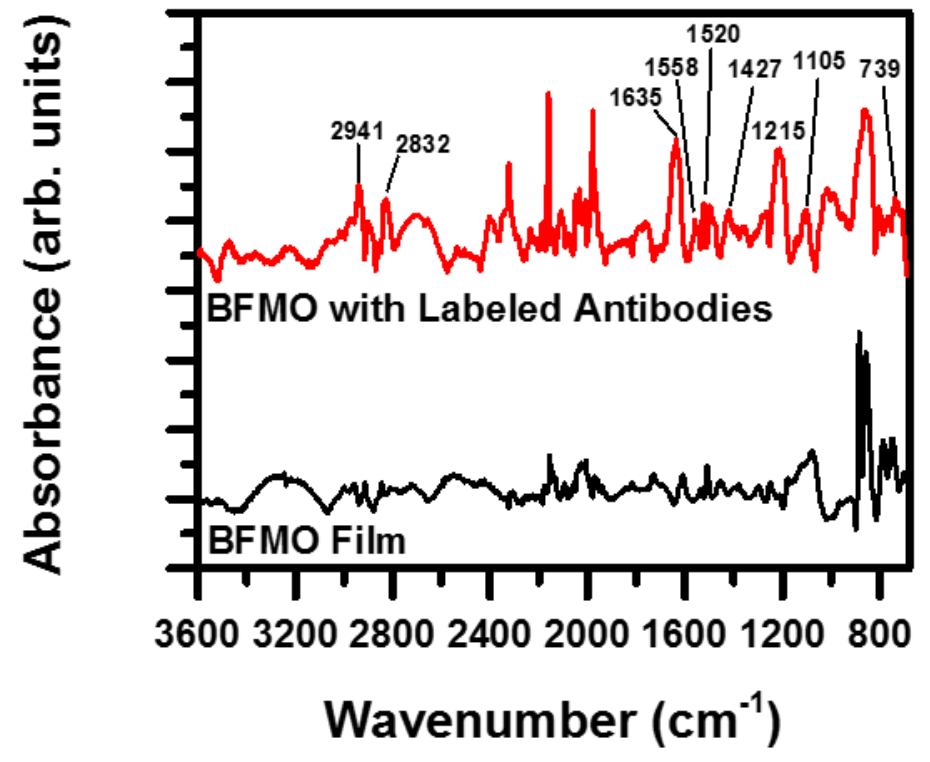

Figure 5.4: Fourier Transform Infrared Transmission spectra of $\mathrm{Bi}_{3} \mathrm{FeMo}_{2} \mathrm{O}_{12}$ (BFMO) film before and after labeling with anti-human IGG antibodies.

From the FTIR spectra in Figure 5.4, several new peaks appear after the human IGG labeling. The new peaks at $739,1520,1558$, and $1635 \mathrm{~cm}-1$ are consistent with the wagging, scissoring, and stretching modes of $\mathrm{N}-\mathrm{H}$ bonds that would be present from amine $\left(-\mathrm{NH}_{2}\right)$ groups on the labeled anti-human IGG antibodies. ${ }^{[24,34]}$ The peaks at 1105 and $1215 \mathrm{~cm}^{-1}$ are consistent with C-N stretching ${ }^{[34]}$ from the linking of the human IGG antibodies to XPS spectra of the BFMO film labeled with anti-human IGG antibodies before conjugation with human IGG (Figure 5.5) further indicates the presence of carboxylic $(-\mathrm{COOH})$ (Figures 5.5a and 5.5c) and amine $\left(-\mathrm{NH}_{2}\right)$ (Figure 5.5b) functional groups consistent with the NHS-EDC linker used to attach the human IGG antibodies to the BFMO and the anti-human IGG antibodies themselves. ${ }^{[35,36]}$ The results are consistent with the FTIR spectra in Figure 5.4. 

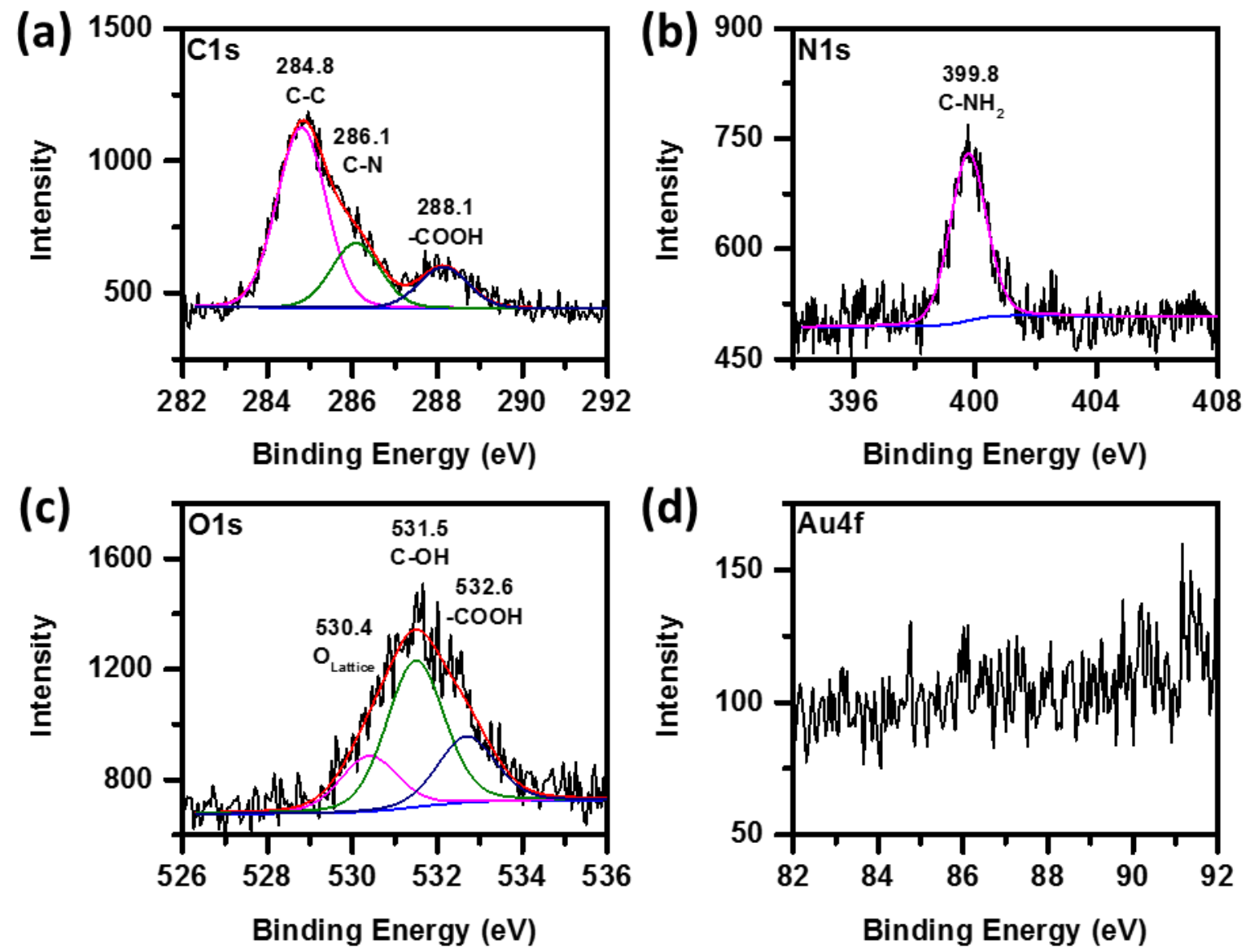

Figure 5.5: XPS Spectra of $\mathrm{Bi}_{3} \mathrm{FeMo}_{2} \mathrm{O}_{12}$ (BFMO) film functionalized with anti-human IGG antibodies before conjugation with IGG and Au NPs; (a) C1s; (b) N1s; (c) O1s; (d) Au4f.

After confirmation of successful anti-human IGG antibody labeling onto the BFMO film, confirmation that the Au NPs could successfully conjugate with the human IGG already conjugated with the anti-human IGG antibody labeled BFMO film is needed. To confirm successful Au NP conjugation, XPS spectra of the anti-human IGG antibody labeled BFMO were collected after Au NPs conjugation (Figure 5.6). 

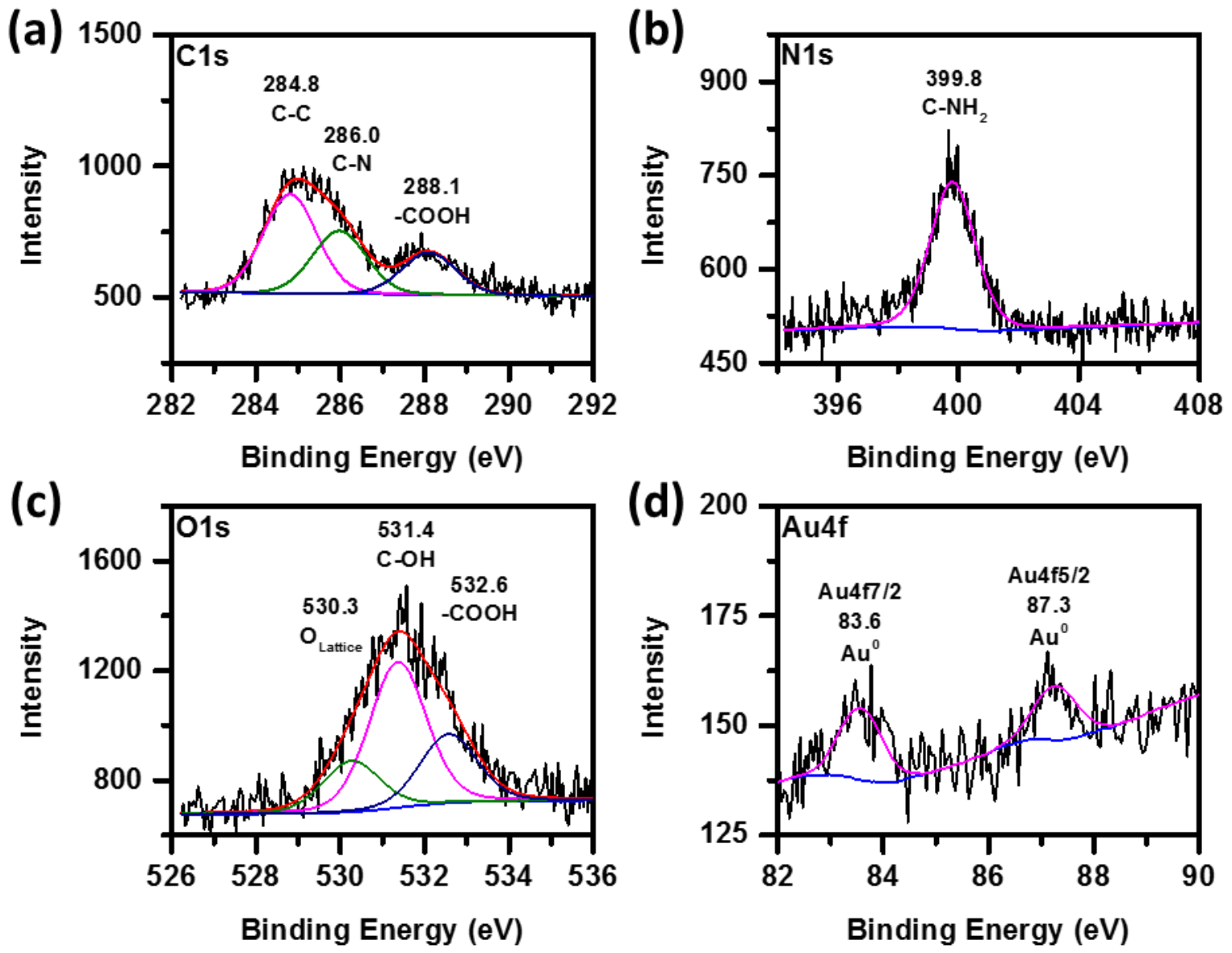

Figure 5.6: XPS Spectra of $\mathrm{Bi}_{3} \mathrm{FeMo}_{2} \mathrm{O}_{12}$ (BFMO) film functionalized with IGG antibodies after conjugation with $200 \mathrm{ng} / \mathrm{mL}$ IGG and Au NPs; (a) C1s; (b) N1s; (c) O1s; (d) Au4f.

After anti-human IGG antibody labeled Au NPs have been conjugated, the C1s, N1s, and O1s (Figures 5.6a through Figures 5.6c) are similar to the XPS spectra obtained before Au NP conjugation, indicating that the surface. However, after Au NP conjugation, a new Au4f signal at $83.6 \mathrm{eV}$ (Figure 5.6d) that was not present before Au NP conjugation (see Figure 5.5d), indicating successful conjugation of the Au NPs ${ }^{[37]}$ After confirming the ability to conjugate Au NPs to the BFMO films utilizes antibody-antigen binding, PEC testing of the BFMO-antibody films and the effects of the human IGG and Au NP conjugation on the PEC performance of the BFMO-antibody films are tested using sample solutions containing $30 \mathrm{ng} / \mathrm{mL}$ of human IGG in PBS buffer. 

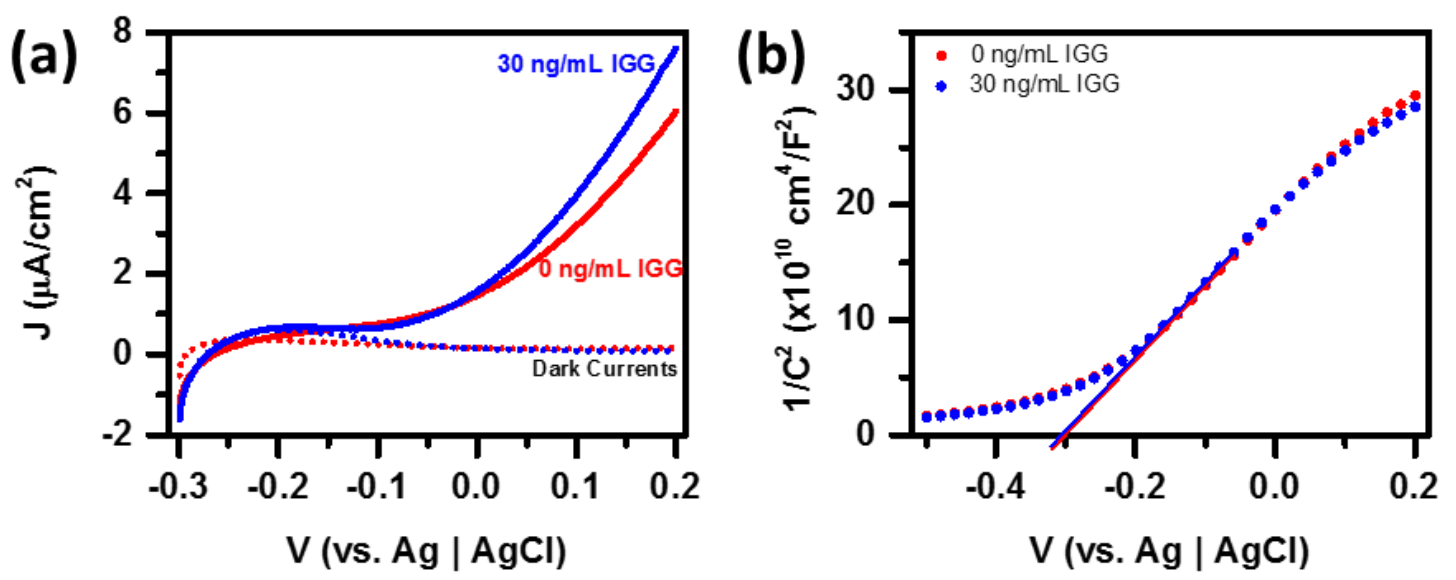

Figure 5.7: (a) Photocurrent-Voltage (J-V) curves and (b) Mott-Schottky (M-S) plots for $\mathrm{Bi}_{3} \mathrm{FeMo}_{2} \mathrm{O}_{12}$ (BFMO) films conjugated with IGG and Au NPs.

Conjugation with a sample solution containing $30 \mathrm{ng} / \mathrm{mL}$ human IGG and then with the antibody labeled $\mathrm{Au}$ NPs results in an increase in photocurrent from $4.48 \mu \mathrm{A} / \mathrm{cm}^{2}$ to $5.65 \mu \mathrm{A} / \mathrm{cm}^{2}$ at $+0.15 \mathrm{~V}$ vs. $\mathrm{Ag} \mid \mathrm{AgCl}$ (Figure 5.7a). The change in photocurrent is sufficient in magnitude to be detectable, showing the potential of this immunosensor design. In addition, conjugation with the human IGG and Au NPs does not result in a change in photocurrent onset potential or flat-band potential as measured from Mott-Schottky plots (Figure 5.7b), indicating that there is no Fermilevel equilibration between the BFMO and Au NPs. ${ }^{[38,39]}$ The lack of Fermi-level equilibration is expected given the lack of contact between the bound Au NPs and the BFMO as the expected separation distance due to the antibody-antigen-antibody sandwich is approximately $10-20 \mathrm{~nm}$ depending on orientation. ${ }^{[40-42]}$ To verify that the mechanism for photocurrent enhancement by the $\mathrm{Au}$ NPs is PIRET, wavelength-dependent incident-photon-to-current efficiency (IPCE) measurements were performed at $+0.15 \mathrm{~V}$ vs. $\mathrm{Ag} \mid \mathrm{AgCl}$ (Figure 5.8).
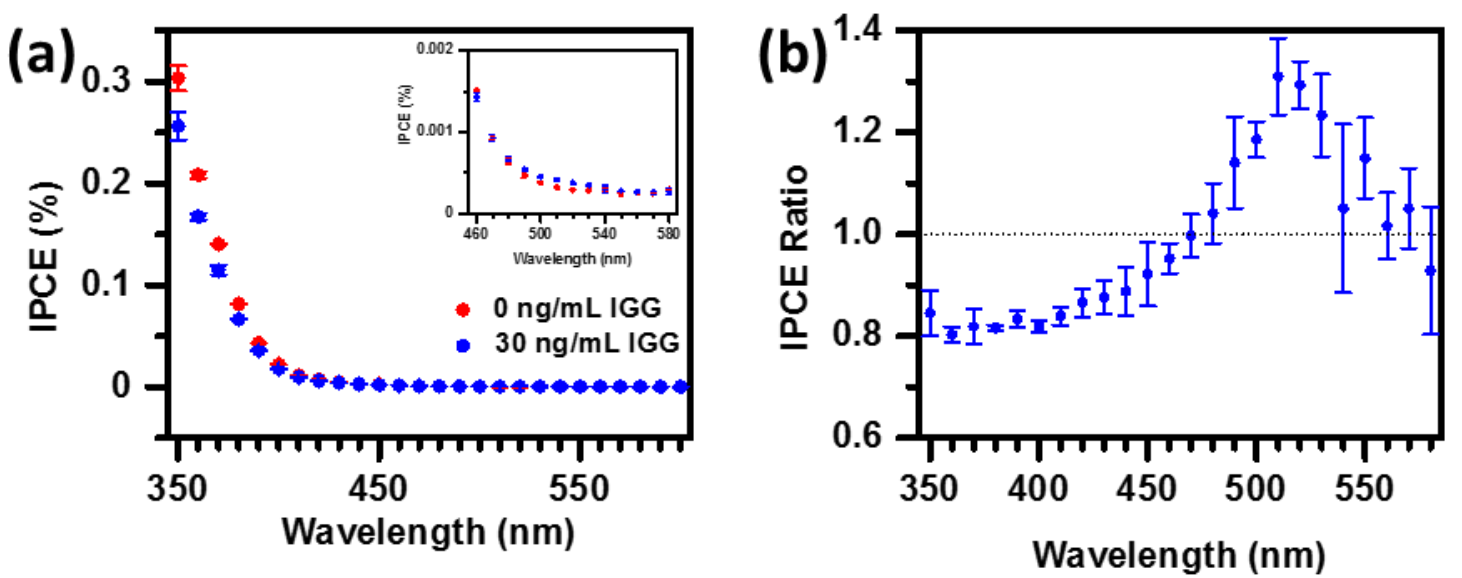

Figure 5.8: (a) Incident-Photon-to-Current Efficiency (IPCE) curves for $\mathrm{Bi}_{3} \mathrm{FeMo}_{2} \mathrm{O}_{12}$ (BFMO) films conjugated with IGG and Au NPs measured at $+0.15 \mathrm{~V}$ vs. Ag $\mid \mathrm{AgCl}$; (b) Ratio of IPCE spectra for BFMO films conjugated with $30 \mathrm{ng} / \mathrm{mL}$ IGG versus $0 \mathrm{ng} / \mathrm{mL}$ IGG. 
From the measured IPCE spectra, there is a decrease in IPCE after conjugation with the 30 $\mathrm{ng} / \mathrm{mL}$ human IGG and Au NPs between $350 \mathrm{~nm}$ and $470 \mathrm{~nm}$. This decrease in IPCE can be attributed primarily to two reasons. First, a slight photoelectrochemical degradation of the BFMO film occurs due to prolonged testing under applied electric bias required for IPCE measurements. In addition, Au NPs absorb light via photoexcited interband transitions between $350-470 \mathrm{~nm}$. ${ }^{[28]}$ The light energy harvested through the Au NPs' interband transitions cannot be transferred to the BFMO film, effectively blocking a portion of usable light from reaching the BFMO. Between 480 $\mathrm{nm}$ and $560 \mathrm{~nm}$, there is an increase in IPCE that correlates well with the LSPR of the Au NPs used for conjugation and the region of spectral overlap between the BFMO and Au NPs. In theory, this enhancement in IPCE between $480-560 \mathrm{~nm}$ could be due to either hot electron injection or PIRET. However, the separation between the conjugated Au NPs and BFMO film due to the antibody-antigen-antibody sandwich prevents electrical contract needed for hot electron injection to be possible. ${ }^{[26-29]}$ As such, the likely photocurrent enhancement mechanism is PIRET as expected. UV-Visible light absorption spectroscopy was performed to see the effects of the conjugated Au NPs on the overall light absorption of the BFMO thin films (Figure 5.9). The UVVisible light absorption spectra (Figure 5.9a) taken before and after conjugation of $100 \mathrm{ng} / \mathrm{mL}$ human IGG and Au NPs shows a small but clear increase in light absorption at wavelengths below $600 \mathrm{~nm}$. Taking the difference in absorption ( $\triangle A b s)$ spectra taken before and after human IGG and $\mathrm{Au}$ NPs conjugation (Figure 5.9b), the difference in absorption matches the expected absorption spectrum shape of Au NPs with a clear peak centered at $530 \mathrm{~nm}$ and rising background consistent with the interband transition of Au. The interband transition absorption at wavelengths below 480 $\mathrm{nm}$ acts as a parasitic absorption that blocks light from the BFMO. Since it has been shown that the photocurrent can be enhanced by the conjugated Au NPs, a sensitivity curve for the BFMO PIRET-based immunosensor is tested (Figure 5.10).
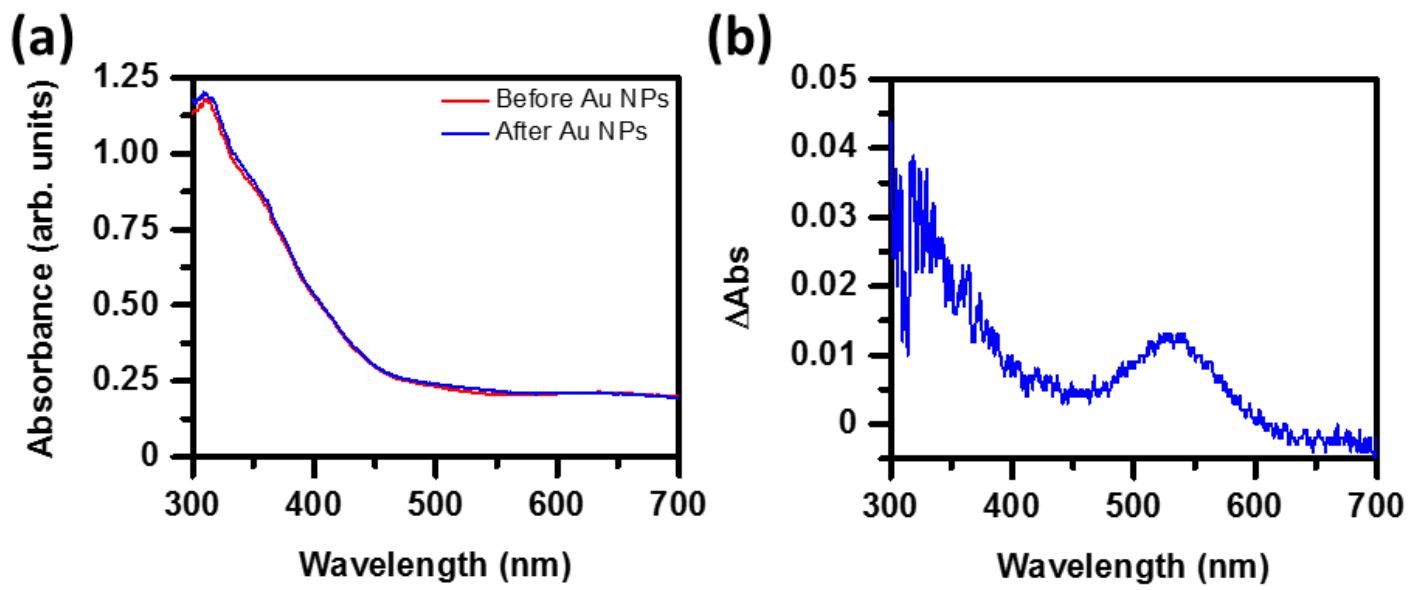

Figure 5.9: (a) UV-Visible light absorbance spectrum of $\mathrm{Bi}_{3} \mathrm{FeMo}_{2} \mathrm{O}_{12}$ (BFMO) film functionalized with IGG antibodies before and after conjugation with $100 \mathrm{ng} / \mathrm{mL}$ IGG and Au NPs; (b) Change in absorbance after conjugation with $100 \mathrm{ng} / \mathrm{mL}$ IGG and Au NPs. 

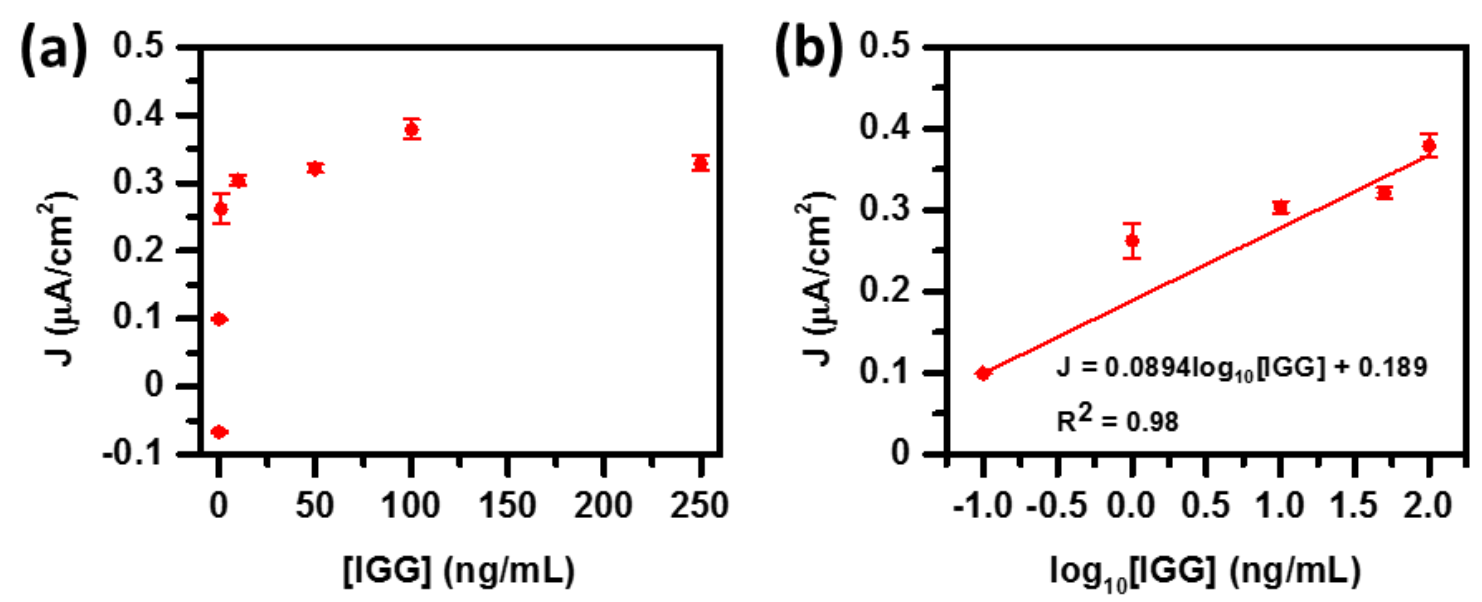

Figure 5.10: (a) Baseline subtracted photocurrent for BFMO Films conjugated with IGG and Au NPs as a function of IGG concentration; (b) Linear $(0.1-250 \mathrm{ng} / \mathrm{mL})$ range of the photocurrent increase curve with linear fit.

From Figure 5.10a, there is a sudden and obvious increase in photocurrent even after small amounts $(100 \mathrm{pg} / \mathrm{mL})$ of human IGG are conjugated to the BFMO-antibody film with the rate of photocurrent increase beginning to level off after concentrations on the order of $10 \mathrm{ng} / \mathrm{mL}$ human IGG. When plotted on a log-linear plot (Figure 5.10b), a mostly linear slope can be seen for the photocurrent increase as a logarithm of the conjugated human IGG concentration up to $250 \mathrm{ng} / \mathrm{mL}$, the highest concentration tested. On the basis of linear slope and standard deviation of measurements in Figure 5.10b (see Section 5.4.8 for the calculation details), a limit of detection of $47 \mathrm{pg} / \mathrm{mL}$ was obtained. However, the magnitude of the photocurrent increase detected in response to conjugated human IGG and Au NPs $\left(\sim 87 \mathrm{nA} / \mathrm{cm}^{2}\right.$ per decade $)$ is low enough that increased noise present during measurements may influence the accuracy of results. Figure 5.11 shows the selectivity of the photocurrent change of the BFMO-IGG-Au photoelectrodes towards human IGG with and without the presence of human anti-mouse antibody and human anti-goat antibody as interference biomolecules commonly found in human blood. 


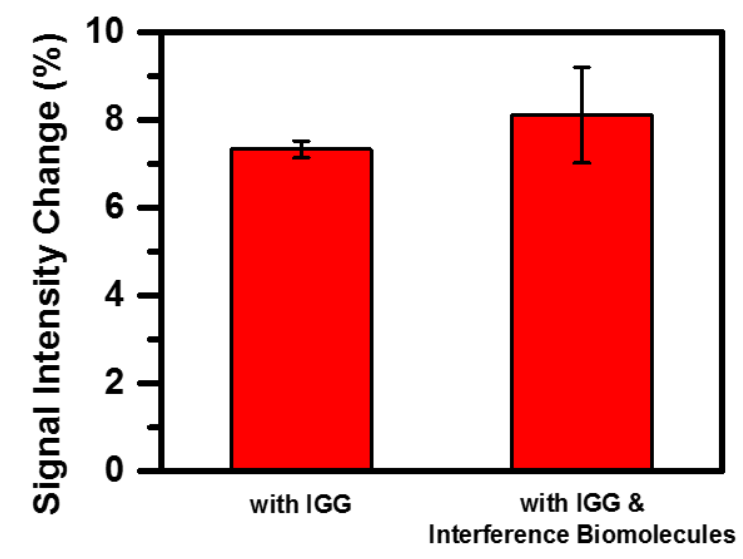

Figure 5.11: Signal intensity change in measured photocurrent signal of the BFMO-IGG-Au in the presence of IGG only, IGG and interference biomolecules (human anti-mouse antibody and human anti-goat antibody), versus photocurrent measured using a $0 \mathrm{ng} / \mathrm{mL}$ human IGG sample as a baseline.

As can be seen from Figure 5.11, there are photocurrent increases $7.33 \pm 0.19 \%$ and 8.10 $\pm 1.09 \%$ increase in photocurrent for the BFMO-IGG-Au photoelectrodes when human IGG and human IGG mixed with the interference biomolecules were incubated with the BFMO-antibody films before $\mathrm{Au}$ NPs respectively. The positive photocurrent increases on the same magnitude when incubating the BFMO-antibody photoelectrodes with either human IGG or the human IGG and the interference biomolecules indicates that the tested interference biomolecules do not interfere with human IGG conjugation to the BFMO-antibody photoelectrodes. This indicates that the BFMO PIRET-based PEC immunosensor is selective towards human IGG as a test analyte with very limited interference from the other biomolecules tested.

The overall low sensitivity of the as-designed BFMO PIRET-based PEC immunosensor is mainly due to the limitations of the materials selected. First, while BFMO has a $2.25 \mathrm{eV}$ bandgap that allows for spectral overlap with the LSPR spherical Au NPs between $480-560 \mathrm{~nm}$, the overall IPCE within this spectral overlap region is low for BFMO in general. The low IPCE is due to a combination of a low absorption coefficient at these wavelengths and poor charge transport due to the formation of small polarons within this spectral window. ${ }^{[32]}$ The main consequence of the low absorption coefficient for BFMO within the spectral overlap region is that it results in a weak acceptor dipole strength that limits the strength of the dipole-dipole interactions that enable PIRET to happen. The dipole-dipole interaction strength is further weakened by the relatively large separation distance $(\sim 10-20 \mathrm{~nm})$ between the conjugated Au NPs and BFMO film as the strength of PIRET has $\mathrm{r}_{\mathrm{o}} / r^{6}$ dependence with $r$ and $r_{o}$ representing the separation distance between the semiconductor acceptor and plasmonic nanoparticle donor and the separation distance between semiconductor and plasmonic nanoparticles where the PIRET efficiency is equal to $50 \% .{ }^{[30,31]}$ Due to the discussed issues with BFMO for application in this PIRET-based PEC immunosensor, a different semiconductor that is more sensitive towards PIRET may be used to replace BFMO along with another plasmonic nanoparticle probe designed such that the separation distance between the plasmonic nanoparticle probe and semiconductor photoelectrode is decreased. 
However, a suitable replacement semiconductor candidate or plasmonic nanoparticle probe design have not been tested. Further research will focus on improving the PIRET-based PEC immunosensor design.

\subsection{Conclusions}

In this chapter, a PEC immunosensor utilizing PIRET from Au NPs conjugated with a BFMO semiconductor thin film. Human IGG was used as a protein biomarker for proof-of-concept testing. BFMO thin films and Au NPs were successfully labeled with anti-human IGG capture antibodies. Successful conjugation of the Au NPs and the BFMO through antibody-antigen reactions was confirmed using XPS. PEC performance testing shows that conjugation of human IGG and the Au NPs to the BFMO thin films enhanced the PEC performance versus BFMO only with the photocurrent enhancement mechanism confirmed as PIRET. Sensitivity testing of the BFMO PIRET-based PEC immunosensor as a function of human IGG concentration showed a low sensitivity $\left(87 \mathrm{nA} / \mathrm{cm}^{2}\right.$ per decade increase in IGG concentration) that was selective to human IGG only. The as-designed PIRET-based PEC immunosensor requires further refinement.

\subsection{Methods}

\subsubsection{Materials}

All chemicals and materials were used as received without further purification. (3aminopropyl)trimethoxysilane (97\%), ammonium hydroxide $\left(28 \% \mathrm{NH}_{3}\right.$ in $\mathrm{H}_{2} \mathrm{O}$ ), bismuth (III) nitrate pentahydrate (ACS, 98\%), hydrochloric acid $(36 \% \mathrm{w} / \mathrm{w})$, hydrogen tetrachloroaurate (III) trihydrate (ACS, 99.99\%), iron (III) nitrate nonahydrate (98+\% metals basis), and trisodium citrate dihydrate (ACS, 99.0\% min) were purchased from Alfa-Aesar. Acetone, citric acid, isopropyl alcohol, reagent alcohol, and sucrose were purchased from VWR International. Triethoxysilylpropyl succinic anhydride (TEPSA) was purchased from Gelest, Inc. 1-ethyl-3-(3(dimethylamino)-propyl) carbodiimide (EDC), bovine serum albumin (BSA), deoxyadenosine triphosphate (dATP), goat anti-human IgG polyclonal antibody, human anti-mouse antibody, human anti-goat antibody, IgG from human serum, N-hydroxysuccinimide (NHS), phosphate buffered saline (PBS, $\mathrm{pH}=7.4$ ) tablets, sodium chloride, sodium dodecyl sulfate (SDS), sodium phosphate $\left(\mathrm{Na}_{3} \mathrm{PO}_{4} \cdot 12 \mathrm{H}_{2} \mathrm{O}\right)$, and Tween 20 were purchased from Sigma-Aldrich. Ammonium molybdate tetrahydrate $\left(\left(\mathrm{NH}_{4}\right)_{6} \mathrm{Mo}_{7} \mathrm{O}_{24} \cdot 4 \mathrm{H}_{2} \mathrm{O}\right)$ was purchased from Ward's Science. Fluorinedoped tin oxide (FTO) coated glass (TEC 15) was purchased from MTI Corporation.

\subsubsection{Characterization}

UV-Visible light absorption spectra were measured using a Shimadzu UV-2550 spectrometer with an integrating sphere (Shimadzu UV 2401/2) using $\mathrm{BaSO}_{4}$ as an optical "white" 
reference material. Field emission scanning electron microscopy (FESEM) was performed using a Hitachi S-4700 FESEM. X-ray diffraction (XRD) spectra were collected using a PANalytical X'pert Pro X-ray diffractometer. Fourier transform infrared (FTIR) spectroscopy was performed using a Thermo Scientific Nicolet 6700 spectrometer using an attenuated total reflection (ATR) accessory. X-ray photoelectron spectroscopy (XPS) was performed using a Physical Electronics Versa Probe 5000. Obtained XPS spectra were corrected for charging using the adventitious carbon $\mathrm{C} 1 \mathrm{~s}$ peak at $284.8 \mathrm{eV}$ as a reference.

\subsubsection{Gold Nanoparticles (Au NPs Synthesis)}

Gold nanoparticles (Au NPs) were synthesized using a traditional citrate reduction method $^{[43,44]}$. In a typical synthesis, $0.0197 \mathrm{~g}$ of hydrogen tetrachloroaurate (III) trihydrate $\left(\mathrm{HAuCl}_{4} \cdot 3 \mathrm{H}_{2} \mathrm{O}\right)$ was dissolved in $100 \mathrm{~mL}$ of DI water. This solution was then heated to boiling. Once the $\mathrm{HAuCl}_{4} \cdot 3 \mathrm{H}_{2} \mathrm{O}$ solution was boiling, $3 \mathrm{~mL}$ of a $1 \%$ (w/v) trisodium citrate dihydrate in DI water solution was added to the $\mathrm{HAuCl}_{4} \cdot 3 \mathrm{H}_{2} \mathrm{O}$ solution where the color changed from light yellow to wine-red over a period of a few minutes. The $\mathrm{HAuCl}_{4} \cdot 3 \mathrm{H}_{2} \mathrm{O} /$ citrate solution was boiled continuously for another 30 minutes and then allowed to cool to room temperature naturally. The as-prepared Au NPs solution was stored for further use without further purification.

\subsection{4 $\mathrm{Bi}_{3} \mathrm{FeMo}_{2} \mathrm{O}_{12}(\mathrm{BFMO})$ Thin Film Synthesis}

$\mathrm{Bi}_{3} \mathrm{FeMo}_{2} \mathrm{O}_{12}$ (BFMO) thin films were synthesized using a modified "Pechini" complex precursor containing $\mathrm{Bi}(\mathrm{III}), \mathrm{Fe}(\mathrm{III})$, and $\mathrm{Mo}(\mathrm{VI})$ salts as metal sources. $2.91 \mathrm{~g}$ of bismuth (III) nitrate pentahydrate and $0.808 \mathrm{~g}$ of iron (III) nitrate were dissolved in $10 \mathrm{~mL}$ of ethylene glycol. Then, $0.706 \mathrm{~g}$ of ammonium molybdate tetrahydrate and $4.61 \mathrm{~g}$ of citric acid were added and dissolved in the precursor solution. The BFMO precursor solution was stirred overnight at room temperature before use.

FTO glass substrates were cleaned by alternating ultrasonication in reagent alcohol, 9\% w/w hydrochloric acid, acetone, and isopropyl alcohol for 20 minutes each followed by treatment under a radio frequency excited oxygen plasma for 90 seconds to ensure substrate hydrophilicity. BFMO deposition onto the cleaned FTO glass substrates was performed via spin coating of the BFMO precursor (4000 RPM, 100 seconds) followed by drying the films at $225^{\circ} \mathrm{C}$ for 15 minutes. The BFMO films were then placed into a muffle furnace and sintered at $650{ }^{\circ} \mathrm{C}$ for 1 hour $(1$ ${ }^{\circ} \mathrm{C} /$ min ramp from $25^{\circ} \mathrm{C}$ to $600{ }^{\circ} \mathrm{C}$ with $10^{\circ} \mathrm{C} / \mathrm{min}$ ramp from $600{ }^{\circ} \mathrm{C}$ to $650{ }^{\circ} \mathrm{C}$; cooling to $25^{\circ} \mathrm{C}$ at $\left.10{ }^{\circ} \mathrm{C} / \mathrm{min}\right)$. 


\subsubsection{Labeling Anti-Human Immunoglobulin G (IGG) Capture Antibody onto BFMO Films}

Anti-human IGG capture antibodies were labeled onto the BFMO thin films based on procedures in literature. ${ }^{[45]}$ The BFMO thin films were first cleaned by successive immersion in ethanol and DI water each for $10 \mathrm{~min}$. The cleaned BFMO films were incubated overnight in an ethanolic solution containing 0.5\% TEPSA and then washed with ethanol to remove free TEPSA. The resulting TEPSA-modified chips were activated by immersion in a PBS solution containing $50 \mathrm{mM}$ NHS and $200 \mathrm{mM}$ EDC. After being washed with PBS solution, chips were incubated overnight in PBS solution containing $1 \mathrm{mg} / \mathrm{mL}$ of anti-human $\mathrm{IgG}$ antibody, followed by rigorously washing with PBS solution to remove free anti-human IgG antibody and kept in a humid chamber prior to assay. The conjugation of antibody onto the chip surface was confirmed by FTIR spectroscopy.

\subsubsection{Labeling Anti-Human IGG Capture Antibody onto Au NPs}

Five milliliters of Au nanospheres were concentrated into one milliliter. Then $0.01 \mathrm{mg}$ of antihuman IgG antibody was added to the concentrated nanoparticles (pH 9.0). The mixture was gently incubated for $1 \mathrm{~h}$ and blocked by $0.1 \mathrm{~mL}$ of $10 \mathrm{wt} \% \mathrm{BSA}$ for $30 \mathrm{~min}$. The resulting solution was centrifuged at 12,500 rpm for $15 \mathrm{~min}$, and the nanoparticles were washed with PBS (1\% BSA) 3 times. The resulting nanoparticles were dispensed in $1.0 \mathrm{~mL}$ of Eluent buffer containing $20 \mathrm{mM}$ $\mathrm{Na}_{3} \mathrm{PO}_{4} \cdot 12 \mathrm{H}_{2} \mathrm{O}, 0.25 \%$ Tween $20,10 \%$ sucrose, and $5 \%$ BSA and stored under $4{ }^{\circ} \mathrm{C}$ for future use.

\subsubsection{Photoelectrochemical (PEC) Testing}

All photoelectrochemical (PEC) testing was performed using a three-electrode cell configuration in a $0.1 \mathrm{M}$ phosphate buffered saline (PBS) $(\mathrm{pH}=7.4)$ aqueous electrolyte. BFMOAntibody thin films with and without $\mathrm{Au}$ NPs functionalization were used as the working electrodes. An $\mathrm{Ag} \mid \mathrm{AgCl}$ electrode (Sat. $\mathrm{KCl} ; \mathrm{E}^{\mathrm{O}}=+0.197 \mathrm{~V}$ vs. NHE) and a platinum mesh were used as the reference and counter electrodes, respectively. All PEC measurements were made using a Gamry Reference 3000 potentiostat/galvanostat/ZRA instrument.

$\mathrm{J}-\mathrm{V}$ curves were recorded using simulated sunlight from a $300 \mathrm{~W}$ Xe arc lamp with an AM1.5G filter calibrated to $100 \mathrm{~mW} / \mathrm{cm}^{2}$ using a thermopile sensor (Newport 818P) as the light source. Wavelength-dependent incident photon-to-current efficiency (IPCE) measurements were performed using light from the $300 \mathrm{~W}$ Xe arc lamp channeled through a monochromator (Oriel 
Cornerstone $^{\mathrm{TM}} 1301 / 8 \mathrm{~m}$ ) as the light source. Wavelength-dependent optical power measurements were performed using a Newport 71675 silicon photodiode detector. The IPCE for a given light wavelength was calculated using Equation $1:{ }^{[46]}$

$$
I P C E=\frac{1240 \mathrm{~J}}{\lambda \cdot P},
$$

where $J$ (in $\left.\mathrm{mA} / \mathrm{cm}^{2}\right)$ is the photocurrent measured under a given light wavelength $(\lambda \mathrm{in} \mathrm{nm})$ and $P$ is the optical power density (in $\mathrm{mW} / \mathrm{cm}^{2}$ ) of the incident light at a given light wavelength.

Mott-Schottky (M-S) plots were obtained at $f=5000 \mathrm{~Hz}$ with an applied AC bias of $10 \mathrm{mV}$ RMS. The obtained electrochemical impedance spectra were used to calculate the space charge capacitance using Equation 2: ${ }^{[47]}$

$$
Z_{i m g}=\frac{1}{2 \pi f C},
$$

where $Z_{i m g}$ is the imaginary component of the measured electrochemical impedance, $f$ is the frequency of the applied AC bias, and $C$ is the space charge capacitance of the sample.

\subsubsection{Human IGG PEC Sensor Testing}

Initially, the baseline PEC performance (J-V curves, M-S, and IPCE) of BFMO films labeled with the anti-human IGG capture antibody. Then, the BFMO films are conjugated with Au NPs in a two-step process. $20 \mu \mathrm{L}$ of target solution containing various human IGG concentrations $(0-250 \mathrm{ng} / \mathrm{mL})$ were dropped onto the detection area of the BFMO-Ab chip. After incubation for 20 min, the BFMO-Ab-IGG film was vigorously rinsed with PBS to remove non-specifically bound human IGG. Then, $20 \mu \mathrm{L}$ of the synthesized anti-human IGG antibody labeled Au NPs were dropped onto the detection area and incubated for $30 \mathrm{~min}$, followed by rinsing with PBS to remove free conjugates. The resulting BFMO-Ab-IGG-Ab-Au film was subjected to the PEC measurements outlined in Section 5.4.7.

The sensitivity of the BFMO-Ab-IGG-Ab-Au photoelectrodes towards human IGG was measured from chronoamperometry (J-t) curves taken at $+0.15 \mathrm{~V}$ vs. $\mathrm{Ag} \mid \mathrm{AgCl}$ using simulated sunlight from $300 \mathrm{~W}$ Xe lamp calibrated to $100 \mathrm{~mW} / \mathrm{cm}^{2}$. The limit of detection of the BFMODNA-Au was determined using Equation $3:{ }^{[48]}$

$$
L O D=10^{\frac{3 \cdot(S D)}{\text { Slope }}},
$$

where $L O D$ is the limit of detection for human IGG, SD is the standard deviation of the photocurrent during measurement of a blank sample ( $0 \mathrm{ng} / \mathrm{mL}$ of human IGG), and Slope is the slope of the linear region of the photocurrent as a function of added human IGG concentration. 


\subsection{References}

[1] Felix, F. S.; Angnes, L. Electrochemical immunosensors - A powerful tool for analytical applications. Biosens. Bioelectron. 2018, 102, 470-478.

[2] Lei, K. F. Electrical detection of sandwich immunoassay on indium tin oxide interdigitated electrodes Micro Nano Lett., 2011, 6, 157-160.

[3] Zhao, W.-W.; Xu, J.-J.; Chen, H.-Y. Photoelectrochemical immunoassays Anal. Chem., 2018, 90, 615-627.

[4] Liu, H.; Rong, P.; Jia, H.; Yang, J.; Dong, B.; Dong, Q.; Yang, C.; Hu, P.; Wang, W.; Liu, H.; Liu, D. A wash-free homogeneous colorimetric immunoassay method Theranostics, 2016, 6, 54-64.

[5] Alamer, S.; Eissa, S.; Chinnappan, R.; Zourob, M. A rapid colorimetric immunoassay for the detection of pathogenic bacteria on poultry processing plants using cotton swabs and nanobeads Microchim. Acta, 2018, 185, 164.

[6] Li, Z.; Wang, Y.; Wang, J.; Tang, Z.; Pounds, J. G.; Lin, Y. Rapid and sensitive detection of protein biomarker using a portable fluorescence biosensor based on quantum dots and a lateral flow test strip Anal. Chem., 2010, 82, 7008-7014.

[7] Chen, R.; Huang, X.; Li, J.; Shan. S.; Lai, W.; Xiong, Y. A novel fluorescence immunoassay for the sensitive detection of Escherichia coli O157:H7 in milk based on catalasemediated fluorescence quenching of CdTe quantum dots Anal. Chim. Acta, 2016, 947, $50-57$.

[8] Gao, X.; Zheng, P.; Kasani, S.; Wu, S.; Yang, F.; Lewis, S.; Nayeem, S.; Engler-Chiurazzi, E. B.; Wigginton, J. G.; Simpkins, J. W.; Wu, N. Paper-based surface-enhanced Raman scattering lateral flow strip for detection of neuron-specific enolase in blood plasma Anal. Chem., 2017, 89, 10104-10110.

[9] Zhang, Y.; Liu, Y.; Li, R.; Khan, M. S.; Gao, P.; Zhang, Y.; Wei, Q. Visible-light driven photoelectrochemical immunosensor based on $\mathrm{SnS}_{2} @ m p g-\mathrm{C}_{3} \mathrm{~N}_{4}$ for detection of prostate specific antigen Sci. Rep., 2017, 7, 4629.

[10] Liu, Y.; Yan, T.; Li, Y.; Cao, W.; Pang, X.; Wu, D.; Wei, Q. A simple label-free photoelectrochemical immunosensor for highly sensitive detection of aflatoxin $\mathrm{B}_{1}$ based on $\mathrm{CdS}-\mathrm{Fe}_{3} \mathrm{O}_{4}$ magnetic nanocomposites $R S C A d v$., 2015, 5, 19581-19586.

[11] An, Y.; Tang, L.; Jiang, X.; Chen, H.; Yang, M.; Jin, L.; Zhang, S.; Wang, C.; Zhang, W. A photoelectrochemical immunosensor based on Au-doped $\mathrm{TiO}_{2}$ nanotube arrays for the detection of $\alpha$-synuclein Chem. Eur. J., 2010, 16, 14439-14446. 
[12] Wang, Y.; Fan, D.; Zhao, G.; Feng, J.; Wei, D.; Zhang, N.; Cao, W.; Du, B.; Wei, Q. Ultrasensitive photoelectrochemical immunosensor for the detection of amyloid $\beta$-protein based on $\mathrm{SnO}_{2} / \mathrm{SnS}_{2} \mathrm{Ag}_{2} \mathrm{~S}$ nanocomposites Biosens. Bioelectron., 2018, 120, 1-7.

[13] Wang, H.; Wang, Y.; Zhang, Y.; Wang, Q.; Ren, X.; Wu, D.; Wei, Q. Photoelectrochemical immunosensor for detection of carcinoembryonic antigen based on $2 \mathrm{D} \mathrm{TiO}_{2}$ nanosheets and carboxylated graphitic carbon nitride Sci. Rep., 2016, 6, 27385.

[14] Shu, J.; Tang, D. Current advances in quantum-dots-based photoelectrochemical immunoassays Chem. Asian J., 2017, 12, 2780-2789.

[15] Li, Y.-J.; Ma, M.-J.; Zhu, J.-J. Dual-signal amplification strategy for ultrasensitive photoelectrochemical immunosensing of $\alpha$-fetoprotein Anal. Chem., 2012, 84, 1049210499.

[16] Haddour, N.; Chauvin, J.; Gondran, C.; Cosnier, S. Photoelectrochemical immunosensor for label-free detection and quantification of anti-cholera toxin antibody J. Am. Chem. Soc., 2006, 128, 9693-9698.

[17] Yang, H.; Zhao, X.; Wang, H.; Deng, W.; Tan, Y.; Ma, M.; Xie, Q. Sensitive photoelectrochemical immunoassay of Staphylococcus aureus based on one-pot electrodeposited $\mathrm{ZnS} / \mathrm{CdS}$ heterojunction nanoparticles Analyst, 2020, 148, 165-171.

[18] Zhao, W.-W.; Chen, R.; Dai, P.-P.; Li, X.-L.; Xu, J.-J.; Chen, H.-Y. A general strategy for photoelectrochemical immunoassay using an enzyme label combined with a $\mathrm{CdS}$ quantum dot/ $/ \mathrm{TiO}_{2}$ nanoparticle composite electrode Anal. Chem., 2014, 86, 1151311516.

[19] Zhu, Y.-C.; Zhang, N.; Ruan, Y.-F.; Zhao, W.-W.; Xu, J.-J.; Chen, H.-Y. Alkaline phosphatase tagged antibodies on gold nanoparticles/TiO2 nanotubes electrode: A plasmonic strategy for label-free and amplified photoelectrochemical immunoassay Anal. Chem., 2016, 88, 5626-5630.

[20] Lv, S.; Zhang, K.; Lin, Z.; Tang, D. Novel photoelectrochemical immunosensor for diseaserelated protein assisted by hemin/G-quadruplex-based DNAzyme on gold nanoparticles to enhance cathodic photocurrent on p- $\mathrm{CuBi}_{2} \mathrm{O}_{4}$ semiconductor Biosens. Bioelectron., 2017, 96, 317-323.

[21] Hu, Y.; Huang, Y.; Wang, Z.; Wang, Y.; Ye, X.; Wong, W.; Li, C.; Sun, D. Gold/WS2 nanocomposites fabricated by in-situ ultrasonication and assembling for photoelectrochemical immunosensing of carcinoembryonic antigen Microchim. Acta, 2018, 185,570 .

[22] Dong, Y.-X.; Cao, J.-T.; Liu, Y.-M.; Ma, S.-H. A novel immunosensing platform for highly sensitive prostate specific antigen detection based on dual-quenching of photocurrent from $\mathrm{CdSe}$ sensitized $\mathrm{TiO}_{2}$ electrode by gold nanoparticles decorated polydopamine nanospheres Biosens. Bioelectron., 2017, 91, 246-252. 
[23] Wang, Y.; Yu, X.; Ye, X.; Wu, K.; Wu, T.; Li, C.; Resonance energy transfer between $\mathrm{ZnCdHgSe} \mathrm{quantum} \mathrm{dots} \mathrm{and} \mathrm{gold} \mathrm{nanorods} \mathrm{enhancing} \mathrm{photoelectrochemical}$ immunosensing of prostate specific antigen Anal. Chim. Acta, 2016, 943, 106-113.

[24] Vashist, S. K.; Schneider, E. M.; Lam, E.; Hrapovic, S.; Luang, J. H. T. One-step antibody immobilization-based rapid and highly sensitive sandwich ELISA procedure for potential in vitro diagnostics Sci. Rep., 2014, 4, 4407.

[25] Lequin, R. M. Enzyme immunoassay (EIA)/enzyme-linked immunosorbent assay (ELISA) Clin. Chem., 2005, 51, 2415-2418.

[26] Tian, Y.; Tatsuma, T., Plasmon-induced photoelectrochemistry at metal nanoparticles supported on nanoporous $\mathrm{TiO}_{2}$ Chem. Commun., 2004, 1810-1811.

[27] Yu, K.; Tian, Y.; Tatsuma, T., Size effects of gold nanoparticles on plasmon-induced photocurrents of gold- $\mathrm{TiO}_{2}$ nanocomposites Phys. Chem. Chem. Phys., 2006, 8, 54175420 .

[28] Clavero, C. Plasmon-induced hot-electron generation at nanoparticle/metal-oxide interfaces for photovoltaic and photocatalytic devices Nat. Photon., 2014, 8, 95-103.

[29] Leenheer, A. J.; Narang, P.; Lewis, N. S.; Atwater, H. A. Solar energy conversion via hot electron internal photoemission in metallic nanostructures: efficiency estimates. J. Appl. Phys. 2014, 115, 134301.

[30] Cushing, S. K.; Li, J.; Meng, F.; Senty, T. R.; Suri, S.; Zhi, M.; Li, M.; Bristow, A. D.; Wu, N. Photocatalytic activity enhanced by plasmonic resonant energy transfer from metal to semiconductor J. Am. Chem. Soc., 2012, 134, 15033-15041.

[31] Li, J.; Cushing, S. K.; Meng, F.; Senty, T. R.; Bristow, A. D.; Wu, N. Plasmon-induced resonance energy transfer for solar energy conversion Nat. Photon., 2015, 9, 601-607.

[32] Liu, B.; Yasin, A. S.; Musho, T.; Bright, J.; Tang, H.; Huang, L.; Wu, N. Visible-light bismuth iron molybdate photocatalyst for artificial nitrogen fixation J. Electrochem. Soc., 2019, 166, H3091-H3096.

[33] Nie, X.; Wulayin, W.; Song, T.; Wu, M.; Qiao, X. Surface, optical characteristics and photocatalytic ability of Scheelite-type monoclinic $\mathrm{Bi}_{3} \mathrm{FeMo}_{2} \mathrm{O}_{12}$ nanoparticles App. Surf. Sci., 2016, 387, 351-357.

[34] Glassford, S. E.; Byrne, B.; Kazarian, S. G. Recent applications of ATR FTIR spectroscopy and imaging to proteins Biochim. Biophys. Acta, 2013, 1834, 2849-2858.

[35] Moulder, J. F.; Stickle, W. F.; Sobol, P. E.; Bomben, K. D. Handbook of X-ray photoelectron spectroscopy; Perkin-Elmer Corporation: 1992; pp 41.

[36] Moulder, J. F.; Stickle, W. F.; Sobol, P. E.; Bomben, K. D. Handbook of X-ray photoelectron spectroscopy; Perkin-Elmer Corporation: 1992; pp 227-228. 
[37] Moulder, J. F.; Stickle, W. F.; Sobol, P. E.; Bomben, K. D. Handbook of X-ray photoelectron spectroscopy; Perkin-Elmer Corporation: 1992; pp 183.

[38] Subramanian, V.; Wolf, E. E.; Kamat, P. V. Catalysis with $\mathrm{TiO}_{2}$ /gold nanocomposites. effect of metal particle size on the Fermi level equilibration. J. Am. Chem. Soc. 2004, 126, 4943-4950.

[39] Li. J.; Cushing, S. K.; Chu, D.; Zheng, P.; Bright, J.; Castle, C.; Manivannan, A.; Wu, N. Distinguishing surface effects of gold nanoparticles from plasmonic effect on photoelectrochemical water splitting by hematite. J. Mater. Res. 2016, 31, 1608-1615.

[40] Wadu-Mesthrige, K; Amro, N. A.; Garno, J. C.; Xu, S.; Liu, G.-Y. Fabrication of nanometer-sized protein patterns using atomic force microscopy and selective immobilization Biophys. J., 2001, 80, 1891-1899.

[41] Reth, M. Matching cellular dimensions with molecular sizes Nat. Immunol., 2013, 14, 765 767.

[42] Sheriff, S.; Silverton, E. W.; Padlan, E. A.; Cohen, G. H.; Smith-Gill, S. J.; Finzel, B. C.; Davies, D. R. Three-dimensional structure of an antibody-antigen complex Proc. Natl. Acad. Sci., 1987, 84, 8075-8079.

[43] Frens, G. Controlled nucleation for the regulation of the particle size in monodisperse gold suspensions. Nat. Phys. Sci. 1973, 241, 20-22.

[44] Kimling, J.; Maier, M.; Okenve, B.; Kotaidis, V.; Ballot, H.; Plech, A. Turkevich method for gold nanoparticle synthesis revisited. J. Phys. Chem. B 2006, 110, 15700-15707.

[45] Zheng, P.; Li, M.; Jurevic, R.; Cushing, S. K.; Liu, Y.; Wu, N. A gold nanohole array based surface-enhanced Raman scattering biosensor for detection of silver (I) and mercury (II) in human saliva. Nanoscale 2015, 7, 11005-11012.

[46] Bak, T.; Nowotny, J.; Rekas, M.; Sorrell, C. C. Photo-electrochemical hydrogen generation from water using solar energy. Materials-related aspects. Int. J. Hydrogen Energy 2002, $27,991-1022$.

[47] Bott, A. W. Electrochemistry of semiconductors. Current Separations 1998, 17, 87-91.

[48] Long, G. L.; Winefordner, J. D. Limit of detection. A closer look at the IUPAC definition. Anal. Chem. 1983, 55, 712-724. 


\section{Chapter 6: Future Outlook and Conclusions}

\subsection{Refining the PIRET Immunosensor Design}

As stated in Chapter 5 of this dissertation, the existing plasmon-induced resonant energy transfer (PIRET)-based PEC immunosensor design based on antibody labeled $\mathrm{Bi}_{3} \mathrm{FeMo}_{2} \mathrm{O}_{12}$ (BFMO) semiconductor thin film coupled to antibody-labeled plasmonic Au nanoparticle (Au NPs) probes using the desired antigen analyte to conjugate them does function as a PEC immunosensor. However, the existing design has low sensitivity in its current form. The sources of the low sensitivity are briefly discussed in Chapter 5, but this discussion will be expanded upon further in this chapter.

When utilizing PIRET to improve light harvesting of the semiconductor as the working mechanism to increase the photocurrent in the PIRET immunosensor design, the semiconductor must still have a finite amount of light absorption within the region of spectral overlap to facilitate the dipole-dipole interactions for PIRET. ${ }^{[1,2]}$ However, despite the need for finite semiconductor light absorption, the balance between light absorption and charge transport within the semiconductor photoelectrode must still be maintained. If the semiconductor film is still optically thin (i.e. not all light is absorbed by the semiconductor film) but physically too thick for all photoexcited charge to be collected, the semiconductor photoelectrode performance will be charge transport limited which PIRET cannot overcome. In the case of the BFMO photoelectrodes used, the dipole-dipole interactions with Au NPs were weak due in part to the low light absorption within the region of spectral overlap with the plasmonic Au nanoparticle probes.

Due to the relative ease of synthesis, spherical Au nanospheres make for an ideal plasmonic nanoparticle probe. If the plasmonic nanoparticle probe is kept as spherical Au NPs $\left(\lambda_{\text {LSPR }} \approx 520\right.$ $\mathrm{nm}$ ), candidate semiconductors to replace BFMO would need to have a bandgap between $2.0 \mathrm{eV}$ $\left(\lambda_{\text {onset }}=620 \mathrm{~nm}\right)$ and $2.5 \mathrm{eV}\left(\lambda_{\text {onset }}=496 \mathrm{~nm}\right)$ to ensure at least partial spectral overlap with the LSPR of the Au NPs. In addition, candidate semiconductors should be either be photoelectrochemically stable in neutral aqueous electrolytes or easily stabilized by charge carrier scavengers or ultra-thin passivation overlayers. Use of charge carrier scavengers or redox couples such as ascorbic acid or ferrocyanide/ferricyanide is already common with PEC sensors due to general photocurrent increases from using readily reduced or oxidized compounds versus difficult to reduce or oxidize compounds like protons or water respectively. ${ }^{[3-11]}$ However, the use of passivation overlayers is not preferable for the PIRET-based PEC immunosensor since the overlayer would be an additional physical barrier that separates the plasmonic nanoprobes and semiconductor photoelectrode that lowers the PIRET efficiency. Based on these guidelines, the following semiconductors may be potential candidates to replace BFMO as the semiconductor in the PIRET-based PEC immunosensor design (Table 6.1): 
Table 6.1: Candidate Semiconductors to Replace $\mathrm{Bi}_{3} \mathrm{FeMo}_{2} \mathrm{O}_{12}$ (BFMO) in the PIRET-based PEC Immunosensor

\begin{tabular}{|c|c|c|c|}
\hline Semiconductor & Bandgap $(\mathbf{e V})$ & $\begin{array}{c}\text { Semiconductor } \\
\text { Type }\end{array}$ & $\begin{array}{c}\text { Stable in Neutral } \\
\text { Aqueous } \\
\text { Electrolyte? }\end{array}$ \\
\hline $\begin{array}{c}\text { Copper (I) Oxide } \\
\left(\mathrm{Cu}_{2} \mathrm{O}\right)^{[12-14]}\end{array}$ & 2.1 & p-type & No \\
\hline $\begin{array}{c}\text { Bismuth Vanadate } \\
\left(\mathrm{BiVO}_{4}\right)^{[15-17]}\end{array}$ & 2.4 & n-type & n-type \\
\hline $\begin{array}{c}\text { Copper (II) } \\
\text { Tungstate } \\
(\mathrm{CuWO})^{[18-20]}\end{array}$ & 2.25 & n-type & No \\
\hline $\begin{array}{c}\text { Cadmium Sulfide } \\
(\mathrm{CdS})^{[4,10,21]}\end{array}$ & 2.4 & & Yes \\
\hline
\end{tabular}

Of the candidate semiconductors in Table 6.1, copper (I) oxide $\left(\mathrm{Cu}_{2} \mathrm{O}\right)$ and cadmium sulfide $(\mathrm{CdS})$ are most commonly used in sensors. PIRET between $\mathrm{Cu} 2 \mathrm{O}$ and $\mathrm{Au}$ NPs has already been studied extensively. As such, there are no mechanistic concerns whether PIRET into $\mathrm{Cu}_{2} \mathrm{O}$ is possible. However, under cathodic PEC operating conditions, $\mathrm{Cu}_{2} \mathrm{O}$ is not stable due to parasitic reduction of the $\mathrm{Cu}_{2} \mathrm{O}$ to metallic $\mathrm{Cu}$ discussed in detail in Chapter 2.3. ${ }^{[12]} \mathrm{Cu}_{2} \mathrm{O}$ can be stabilized at least in part using passivation overlayers and using electron scavengers such as methyl viologen or hydrogen peroxide. ${ }^{[1,14]}$ However, the stability of the labeled antibodies in the presence of methyl viologen and hydrogen peroxide must be further studied. Cadmium sulfide is commonly utilized in PEC sensors including those based on Förster resonant energy transfer (FRET) with spherical Au NPs. ${ }^{[4,10]}$ CdS can also be readily stabilized using hole scavengers such as sodium sulfite, sodium sulfide, and ascorbic acid. ${ }^{[21]}$ However, the ease of FRET between CdS and Au NPs is also problematic for use a PIRET sensor since the direction of FRET counters any photocurrent enhancement that would occur from PIRET. ${ }^{[2,4]}$ In addition, CdS utilizes cadmium, a heavy metal, as a constituent material. The toxicity of cadmium should limit the use of CdS to controlled settings where the cadmium can be contained. Bismuth vanadate $\left(\mathrm{BiVO}_{4}\right)$ and copper (II) tungstate $\left(\mathrm{CuWO}_{4}\right)$ are both oxide semiconductors that have been heavily studied for PEC water-splitting for solar energy harvesting with limited use for sensing. $\mathrm{BiVO}_{4}$ is one of the most promising material for PEC water-splitting. In addition, PIRET between spherical Au NPs and $\mathrm{BiVO}_{4}$ has been previously observed. ${ }^{[16,17]}$ However, $\mathrm{BiVO}_{4}$ is not stable in neutral aqueous electrolytes without either a passivation overlayer or a hole scavenger. $\mathrm{CuWO}_{4}$ is stable in neutral aqueous borate buffers but not in neutral aqueous phosphate buffers. ${ }^{[18]}$ However, there is limited study on plasmonic energy transfer between plasmonic nanoparticles and $\mathrm{CuWO}_{4} \cdot{ }^{[20]}$ Nonetheless, both $\mathrm{BiVO}_{4}$ and $\mathrm{CuWO}_{4}$ are potential replacement photoelectrode materials for consideration.

The large separation distance $(\sim 10-20) \mathrm{nm}$ ) between the BFMO thin film and plasmonic Au nanoparticle probes resulting from the antibody-antigen-antibody sandwich that conjugates the BFMO and Au NPs together. ${ }^{[22-24]}$ As described previously, PIRET is a resonant energy transfer process that utilizes dipole-dipole interactions to transfer energy from a plasmonic nanoparticle 
donor to a nearby semiconductor acceptor. While PIRET does not require direct contact between the semiconductor and plasmonic nanoparticle to occur, the efficiency of PIRET falls off with a $\left(r_{o} / r^{6}\right)$ dependence with $r$ and $r_{o}$ representing the separation distance between the semiconductor acceptor and plasmonic nanoparticle donor and the separation distance between semiconductor and plasmonic nanoparticles where the PIRET efficiency is equal to $50 \%{ }^{[2]}$ While $r_{o}$ is an empirically derived semiconductor material dependent constant typically on the order of $\sim 10 \mathrm{~nm}$, there is a rapid drop-off in the PIRET efficiency as the separation distance increases. The practical separation distance of 10-20 nm from the antibody-antigen-antibody sandwich is too much for efficient PIRET to occur between the BFMO and Au NPs in the existing PIRET immunosensor design. It should be noted that this is a similar problem for signal "turn-off" PEC immunosensors based on signal quenching by Förster resonant energy transfer (FRET) between conjugated plasmonic nanoparticles and a semiconductor photoelectrode as a working mechanism. Similar approaches for overcoming the separation distance problem in FRET-based PEC immunosensors may be applicable to the PIRET immunosensor.

A possible solution to reduce the separation distance would be to create a plasmonic nanoprobe based on plasmonic nanoparticles conjugated with single-strand DNA-antibody (ssDNA-Ab) conjugates rather than only to antibodies (Figure 6.1). Such ssDNA-AB conjugates are already studied for their applications for immuno-PCR, a technique used for protein detection. ${ }^{[26,27]}$ In the proposed plasmonic nanoparticle probes, long single stranded DNA strands would act as flexible tethers that allow conjugated Au NPs to rest very near or in contact with the semiconductor photoelectrode. This approach is similar in concept to the use of compatible ssDNA of mismatched length conjugated to Au NPs utilized for the PEC sensor for $\mathrm{Hg}^{2+}$ detection presented in Chapter 4 of this dissertation. The resulting reduced separation distance between the plasmonic nanoprobe and semiconductor film should greatly increase the PIRET efficiency while hopefully offsetting additional steric hinderance from the additional bulk of the ssDNA-Ab conjugates. 


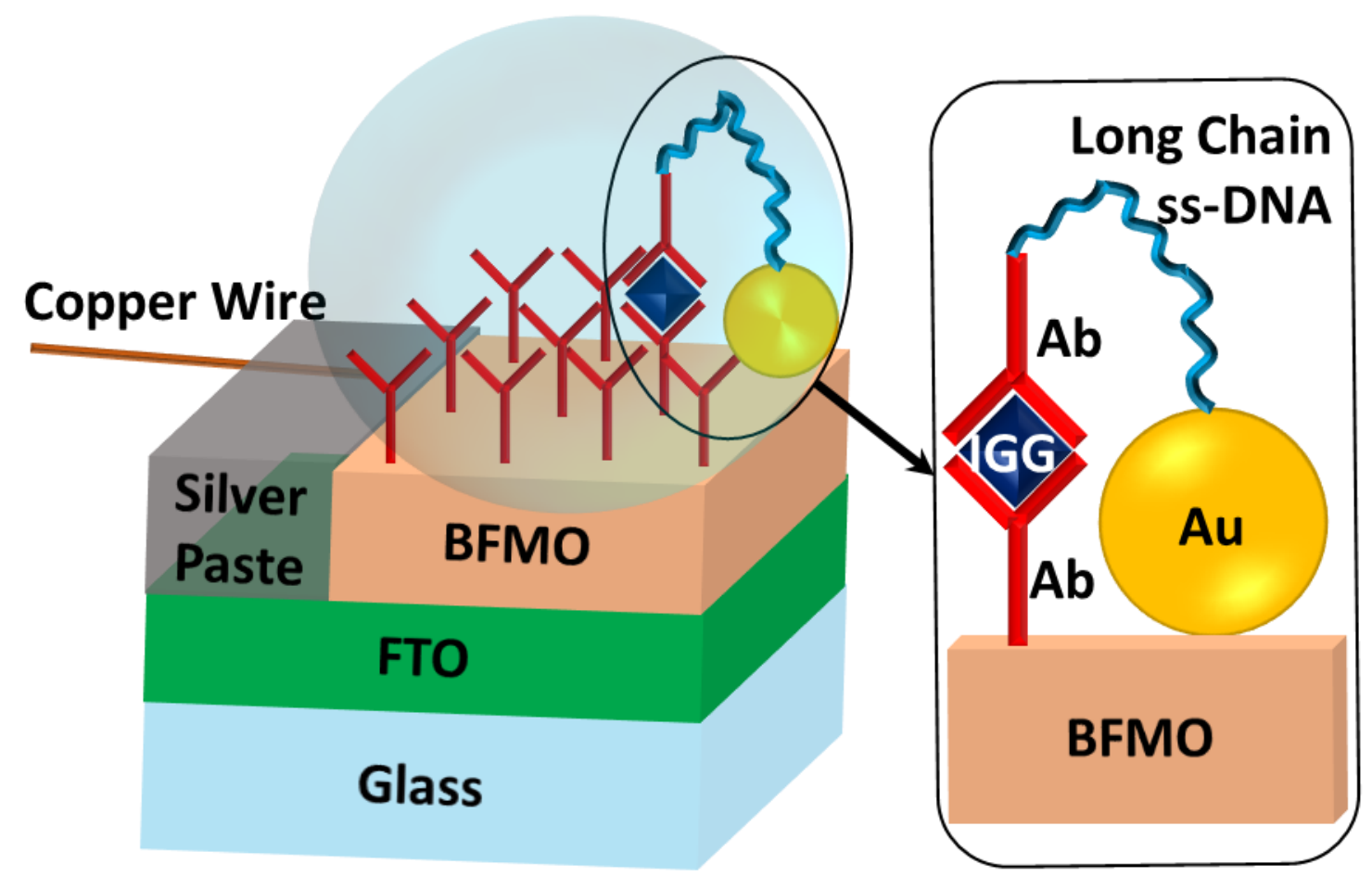

Figure 6.1: Proposed replacement plasmonic Au NP nanoprobe conjugate system for conjugating $\mathrm{Au}$ NPs to $\mathrm{Bi}_{3} \mathrm{FeMo}_{2} \mathrm{O}_{12}$ (BFMO)semiconductor film for PIRET-based PEC immunosensor.

\subsection{Conclusions}

In this dissertation, three photoelectochemistry (PEC) based projects utilizing semiconductor photoelectrodes are presented for applications related to solar energy harvesting and sensing. In the first presented project, a titanium dioxide $\left(\mathrm{TiO}_{2}\right)$ nanorod array was coated with an ultra-thin zirconium and $\mathrm{Co}^{3+}$ infiltrated porphyrin-based metal-organic framework (MOF) known as PCN-225 for use as an improved photoanode for solar water-splitting. The $\mathrm{TiO}_{2}$ nanorod array coated with the $\mathrm{Co}^{3+}$ infiltrated PCN-225 MOF $\left(\mathrm{TiO}_{2} @ \mathrm{Co}-\mathrm{MOF}\right)$ exhibited enhanced PEC performance for solar water-splitting. Despite the wide absorption spectrum of the PCN-225 MOF, the enhanced solar-water splitting performance of the $\mathrm{TiO}_{2} @ \mathrm{Co}-\mathrm{MOF}$ was confined to light illumination wavelengths where the $\mathrm{TiO}_{2}$ nanorod array already absorbed rather than extending the spectrum of useful light wavelengths. The photocurrent enhancement from the $\mathrm{Co}^{3+}$ infiltrated PCN-225 MOF coating is due to improved charge separation from the suppression of surface states on the $\mathrm{TiO}_{2}$ and the built-in potential from a depleted p-n junction formed between the PCN-225 and $\mathrm{TiO}_{2}$. This research further shows the potential of thin surface coatings for improving the PEC performance and the difficulties in utilizing metal-organic frameworks for efficient PEC watersplitting. 
In the second and third projects presented, a phenomenon known as localized surface plasmon resonance (LSPR) that occurs with nanoparticles of certain metals such as $\mathrm{Au}$ and $\mathrm{Ag}$ is utilized to modulate the PEC response of $\mathrm{Bi}_{3} \mathrm{FeMo}_{2} \mathrm{O}_{12}$ (BFMO) semiconductor photoelectrodes for sensing applications. In the second presented project, the presence of plasmonic energy transfer mechanisms such as hot electron injection and plasmon-induced resonant energy transfer (PIRET) as signal "turn-on" mechanisms for use in PEC sensing. Single-strand DNA (ss-DNA) and $\mathrm{Hg}^{2+}$ ions' affinity for the thymine bases on DNA were used to conjugate plasmonic Au NPs and $\mathrm{Au} @ \mathrm{SiO}_{2}$ core-shell NPs to BFMO semiconductor thin-film photoelectrodes. When Au NPs were conjugated with the BFMO thin-film, a combination of hot electron injection, PIRET, wavelengthindependent Fermi-level equilibration, and a wavelength-dependent internal reflection that increased light absorption within the BFMO photoelectrode increased the photocurrent of the BFMO photoelectrode. When $\mathrm{Au} @ \mathrm{SiO}_{2} \mathrm{NPs}$ were conjugated to the BFMO thin-film, the thin insulating $\mathrm{SiO}_{2}$ layer blocked hot electron injection and prevented Fermi level equilibration while PIRET and the wavelength dependent internal reflection still occurred. A PEC sensor for $\mathrm{Hg}^{2+}$ detection was created using bare Au NPs as the plasmonic probe and demonstrated a sensitivity and selectivity for detection of $\mathrm{Hg}^{2+}$ ions in water sufficient to meet detection requirements for safe drinking and waste-water limits on $\mathrm{Hg}^{2+}$ ions. levels. The results from this project highlight the promise that plasmonic energy transfer mechanisms have as a method to modulate the PEC response of photoelectrodes for sensing applications.

In the third project, the proof-of-concept for a PEC immunosensor for human immunoglobulin $\mathrm{G}(\mathrm{IgG})$ detection utilizing PIRET from plasmonic Au NP nanoparticle-based probes to a BFMO semiconductor thin film as the working mechanism for photocurrent modulation. The resulting PIRET-based PEC immunosensor did exhibit a wavelength-dependent photocurrent enhancement consistent with PIRET between the Au nanoprobes and the BFMO thin film. However, while the PEC immunosensor functions, the sensor exhibited low sensitivity towards human IgG due to low PIRET efficiency between the conjugated Au NPs and BFMO thin film. The low PIRET efficiency is attributed to low light absorption of the BFMO within the spectral overlap with the LSPR of the spherical Au NPs leading to low dipole-dipole interaction strength and a relatively large separation distance $(10-20 \mathrm{~nm})$ between the BFMO and conjugated $\mathrm{Au}$ NPs resulting from the size of the antibody-antigen-antibody sandwich used for conjugation. As such, further optimization of the BFMO semiconductor photoelectrode and the design of the plasmonic Au NP probes is needed to improve the PIRET-based PEC immunosensor design.

While research on PEC for solar energy harvesting applications has stagnated due to insufficient progress towards reaching efficiency and cost requirements to be competitive on the energy market, PEC for sensing applications is an alive and growing field. The potential growth in the PEC-based sensor field is due to two reasons. The first reason is that light conversion efficiency is less important than the ability to modulate the PEC performance of a photoelectrode directly or indirectly in response to the presence of an analyte for sensing applications. This allows for use of less expensive materials such as metal oxide semiconductors for the photoelectrode that may not produce as much photocurrent as more expensive III-V semiconductors. The second reason is the low equipment requirements (potentiostat, light source, and a transparent PEC cell) and technical expertise required to setup and operate the PEC experiments used for sensing. This 
dissertation has focused on utilizing plasmonic photocurrent enhancement mechanisms (PIRET and hot electron injection) as a photocurrent modulation mechanism for the signal "turn-on" type of PEC sensor. While the presented results are promising, further design optimization particularly in the case of the PIRET-based PEC immunosensor is necessary for creating a sensor with the sensitivity and limit of detection required for current analytes of interest.

\subsection{References}

[1] Cushing, S. K.; Li, J.; Meng, F.; Senty, T. R.; Suri, S.; Zhi, M.; Li, M.; Bristow, A. D.; Wu, N. Photocatalytic activity enhanced by plasmonic resonant energy transfer from metal to semiconductor J. Am. Chem. Soc., 2012, 134, 15033-15041.

[2] Li, J.; Cushing, S. K.; Meng, F.; Senty, T. R.; Bristow, A. D.; Wu, N. Plasmon-induced resonance energy transfer for solar energy conversion Nat. Photon., 2015, 9, 601-607.

[3] Wang, Y.; Yu, X.; Ye, X.; Wu, K.; Wu, T.; Li, C.; Resonance energy transfer between $\mathrm{ZnCdHgSe} \mathrm{quantum} \mathrm{dots} \mathrm{and} \mathrm{gold} \mathrm{nanorods} \mathrm{enhancing} \mathrm{photoelectrochemical}$ immunosensing of prostate specific antigen Anal. Chim. Acta, 2016, 943, 106-113.

[4] Han, D.-M.; Jiang, L.-Y.; Tang, W.-Y.; Xu, J.-J.; Chen, H.-Y. Photoelectrochemical determination of inorganic mercury ions based on energy transfer between CdS quantum dots and Au nanoparticles Electrochem. Comm., 2015, 51, 72-75.

[5] Li, Y.-J.; Ma, M.-J.; Zhu, J.-J. Dual-signal amplification strategy for ultrasensitive photoelectrochemical immunosensing of $\alpha$-fetoprotein Anal. Chem., 2012, 84, 1049210499.

[6] Zhao, W.-W.; Chen, R.; Dai, P.-P.; Li, X.-L.; Xu, J.-J.; Chen, H.-Y. A general strategy for photoelectrochemical immunoassay using an enzyme label combined with a CdS quantum dot/ $\mathrm{TiO}_{2}$ nanoparticle composite electrode Anal. Chem., 2014, 86, 1151311516.

[7] An, Y.; Tang, L.; Jiang, X.; Chen, H.; Yang, M.; Jin, L.; Zhang, S.; Wang, C.; Zhang, W. A photoelectrochemical immunosensor based on Au-doped $\mathrm{TiO}_{2}$ nanotube arrays for the detection of $\alpha$-synuclein Chem. Eur. J., 2010, 16, 14439-14446.

[8] Wang, Y.; Fan, D.; Zhao, G.; Feng, J.; Wei, D.; Zhang, N.; Cao, W.; Du, B.; Wei, Q. Ultrasensitive photoelectrochemical immunosensor for the detection of amyloid $\beta$-protein based on $\mathrm{SnO}_{2} / \mathrm{SnS}_{2} \mathrm{Ag}_{2} \mathrm{~S}$ nanocomposites Biosens. Bioelectron., 2018, 120, 1-7.

[9] Wang, H.; Wang, Y.; Zhang, Y.; Wang, Q.; Ren, X.; Wu, D.; Wei, Q. Photoelectrochemical immunosensor for detection of carcinoembryonic antigen based on $2 \mathrm{D}^{\mathrm{TiO}_{2}}$ nanosheets and carboxylated graphitic carbon nitride Sci. Rep., 2016, 6, 27385. 
[10] Liu, Y.; Yan, T.; Li, Y.; Cao, W.; Pang, X.; Wu, D.; Wei, Q. A simple label-free photoelectrochemical immunosensor for highly sensitive detection of aflatoxin $\mathrm{B}_{1}$ based on $\mathrm{CdS}-\mathrm{Fe}_{3} \mathrm{O}_{4}$ magnetic nanocomposites $R S C A d v$., 2015, 5, 19581-19586.

[11] Zhang, Y.; Liu, Y.; Li, R.; Khan, M. S.; Gao, P.; Zhang, Y.; Wei, Q. Visible-light driven photoelectrochemical immunosensor based on $\mathrm{SnS}_{2} @$ mpg- $\mathrm{C}_{3} \mathrm{~N}_{4}$ for detection of prostate specific antigen Sci. Rep., 2017, 7, 4629.

[12] Paracchino, A.; Brauer, J. C.; Moser, J.-E.; Thimsen, E.; Graetzel, M. Synthesis and characterization of high-photoactivity electrodeposited $\mathrm{Cu}_{2} \mathrm{O}$ solar absorber by photoelectrochemistry and ultrafast spectroscopy J. Phys. Chem. C., 2012, 116, 73417350 .

[13] Luo, J.; Steier, L.; Son, M.-K.; Schreier, M.; Mayer, M. T.; Grätzel, M. $\mathrm{Cu}_{2} \mathrm{O}$ nanowire photocathodes for efficient and durable solar water splitting Nano Lett., 2016, 16, 18481857.

[14] Somasundaram, S.; Chenthamarakshan, C. R. N.; de Tacconi, N. R.; Rajeshwar, K. Photocatalytic production of hydrogen from electrodeposited $\mathrm{p}-\mathrm{Cu}_{2} \mathrm{O}$ film and sacrificial electron donors Int. J. Hydrog. Ener., 2007, 32, 4661-4669.

[15] Abdi, F. F.; van de Krol, R. Nature and light dependence of bulk recombination in Co-Picatalyzed $\mathrm{BiVO}_{4}$ photoanodes J. Phys. Chem. C, 2012, 116, 9398-9404.

[16] Abdi, F. F.; Dabirian, A.; Dam, B.; van de Krol, R. Plasmonic enhancement of the optical absorption and catalytic efficiency of $\mathrm{BiVO}_{4}$ photoanodes decorated with $\mathrm{Ag} @ \mathrm{SiO}_{2}$ coreshell nanoparticles Phys. Chem. Chem. Phys., 2014, 16, 15272-15277.

[17] Zhang, L.; Lin, C.-Y.; Valev, V. K.; Reisner, E.; Steiner, U.; Baumberg, J. J. Plasmonic enhancement in $\mathrm{BiVO}_{4}$ photonic crystals for efficient water splitting Small, 2014, 10, 3970-3978.

[18] Yourey, J. E.; Pyper, K. J.; Kurtz, J. B.; Bartlett, B. M. Chemical stability of CuWO $\mathrm{Cu}_{4}$ for photoelectrochemical water oxidation J. Phys. Chem. C, 2013, 117, 8708-8718.

[19] Gao, Y.; Hamann, T. W. Quantitative hole collection for photoelectrochemical water oxidation with $\mathrm{CuWO}_{4}$ Chem. Commun., 2017, 53, 1285-1288.

[20] Valenti, M.; Dolat, D.; Biskos, G.; Schmidt-Ott, A.; Smith, W. A. Enhancement of the photoelectrochemical performance of $\mathrm{CuWO}_{4}$ thin films for solar water splitting by plasmonic nanoparticle functionalization J. Phys. Chem. C, 2015, 119, 2096-2104.

[21] Li, J.; Cushing, S. K.; Zheng, P.; Senty, T.; Meng, F.; Bristow, A. D.; Manivannan, A.; Wu, N. Solar hydrogen generation by a CdS-Au-TiO 2 sandwich nanorod array enhanced with Au nanoparticle as electron relay and plasmonic photosensitizer J. Am. Chem. Soc., 2014, 136, 8438-8449. 
[22] Wadu-Mesthrige, K; Amro, N. A.; Garno, J. C.; Xu, S.; Liu, G.-Y. Fabrication of nanometer-sized protein patterns using atomic force microscopy and selective immobilization Biophys. J., 2001, 80, 1891-1899.

[23] Reth, M. Matching cellular dimensions with molecular sizes Nat. Immunol., 2013, 14, 765767.

[24] Sheriff, S.; Silverton, E. W.; Padlan, E. A.; Cohen, G. H.; Smith-Gill, S. J.; Finzel, B. C.; Davies, D. R. Three-dimensional structure of an antibody-antigen complex Proc. Natl. Acad. Sci., 1987, 84, 8075-8079.

[25] Fan, D.; Wu, D.; Cui, J.; Chen, Y.; Ma, H.; Liu, Y.; Wei, Q.; Du, B. An ultrasensitive labelfree immunosensor based on $\mathrm{CdS}$ sensitized $\mathrm{Fe}-\mathrm{TiO}_{2}$ with high visible-light photoelectrochemical activity Biosens. Bioelectron., 2015, 74, 843-848.

[26] Kazane, S. A.; Sok, D.; Cho, E. H.; Uson, M. L.; Kuhn, P.; Schultz, P. G.; Smider, V. V. Site-specific DNA-antibody conjugates for specific and sensitive immuno-PCR Proc. Natl. Acad. Sci. U. S. A., 2012, 109, 3731-3736.

[27] van Buggenum, J. A. G. L.; Gerlach, J. P.; Eising, S.; Schoonen, L.; van Eijl, R. A. P. M.; Tanis, S. E. J.; Hogeweg, M.; Hubner, N. C.; van Hest, J. C.; Bonger, K. M.; Mulder, K. W. A covalent and cleavable antibody-DNA conjugation strategy for sensitive protein detection via immuno-PCR Sci. Rep., 2016, 6, 22675. 


\section{Appendix: Manuscripts and Publications}

\section{Completed Manuscripts}

1. Comparison of Plasmon-based Photocurrent Enhancement Mechanisms for Use in Photoelectrochemical Sensors

Joeseph Bright, Xuefei Gao, Jennifer Boryczka, Kathrine Curtin, Nianqiang Wu

2. Multifaceted Roles of Gold Nanoparticles in Light Energy Harvesting and Conversion Devices

Joeseph Bright and Nianqiang Wu

\section{Publications (* Co-First Authorship)}

1. "A Single-Ion Conducting UiO-66 Metal-Organic Framework Electrolyte for All-SolidState Lithium Batteries"

ACS Applied Energy Materials, 2020, Accepted Manuscript

Hui Yang, Botong Liu, Joeseph Bright, Sujan Kasani, Jianhui Yang, Xiangwu Zhang, and Nianqiang $\mathrm{Wu}$

2. "Chemical Interaction and Enhanced Interfacial Ion Transport in a Ceramic NanofiberPolymer Composite Electrolyte for All-Solid-State Lithium Metal Batteries"

Journal of Materials Chemistry A, 2020, 8, 7261-7272

Hui Yang, Joeseph Bright, Banghao Chen, Peng Zheng, Xuefei Gao, Botong Liu, Sujan Kasani, Xiangwu Zhang, and Nianqiang Wu

3. "Tunable Visible-Light Surface Plasmon Resonance of Molybdenum Oxide Thin Films Fabricated by E-beam Evaporation"

ACS Applied Electronic Materials, 2019, 1, 11, 2389-2395.

Sujan Kasani, Peng Zheng, Joeseph Bright, and Nianqiang Wu

4. "Functionalization of a Metal-Organic Framework Semiconductor for Tuned Band Structure and Catalytic Activity"

Journal of the Electrochemical Society, 2019, 155, 5 H3029-H3034

Jiangtian Li, Terence Musho, Joeseph Bright, and Nianqiang Wu

5. Metal-organic Framework Coated Titanium Dioxide Nanorod Array p-n Heterojunction Photoanode for Solar Water-Splitting

Nano Research, 2019, 12, 3, 643-650

Hui Yang, Joeseph Bright*, Sujan Kasani, Peng Zheng, Terence Musho, Banglin Chen, Ling Huang, Nianqiang $\mathrm{Wu}$ 
6. "Visible-Light Bismuth Iron Molybdate Photocatalyst for Artificial Nitrogen Fixation" Journal of the Electrochemical Society, 2019, 155, 5, H3091-H3096 Botong Liu, Alhassan S. Yasin, Terence Musho, Joeseph Bright, Haibin Tang, Ling Huang, and Nianqiang $\mathrm{Wu}$

7. "Effects of Defects on Photocatalytic Activity of Hydrogen-Treated Titanium Oxide Nanobelts" ACS Catalysis, 2017, 7, 3, 1742-1748

Scott K. Cushing, Fanke Meng, Junying Zhang, Bangfu Ding, Chih Kai Chen, Chih-Jung Chen, Ru-Shi Liu, Alan D. Bristow, Joeseph Bright, Peng Zheng, and Nianqiang Wu

8. "Distinguishing Surface Effects of Gold Nanoparticles from Plasmonic Effect on Photoelectrochemical Water Splitting by Hematite"

Journal of Materials Research, 2016, 31, 11, 1608-1615

Jiangtian Li, Scott K. Cushing, Deryn Chu, Peng Zheng, Joeseph Bright, Conner Castle, Ayyankkanu Manivannan, and Nianqiang Wu

9. "Controlling Plasmon-Induced Resonance Energy Transfer and Hot Electron Injection Processes in Metal@ $\mathrm{TiO}_{2}$ Core-Shell Nanoparticles"

Journal of Physical Chemistry C, 2015, 119, 28, 16239-16244

Scott K. Cushing, Jiangtian Li, Joeseph Bright, Brandon T. Yost, Peng Zheng, Alan D. Bristow, and Nianqiang $\mathrm{Wu}$

10. "Investigation of Band Gap Narrowing in Nitrogen-Doped $\mathrm{La}_{2} \mathrm{Ti}_{2} \mathrm{O}_{7}$ with Transient Absorption Spectroscopy" Physical Chemistry Chemical Physics, 2015, 17, 31039-31043

Brandon T. Yost, Scott K. Cushing, Fanke Meng, Joeseph Bright, Derek A. Bas, Nianqiang $\mathrm{Wu}$, and Alan D. Bristow

11. "Photocatalytic Water Oxidation by Hematite/Reduced Graphene Oxide Composites" ACS Catalysis, 2013, 3, 4, 746-751

Fanke Meng, Jiangtian Li, Scott K. Cushing, Joeseph Bright, Mingjia Zhi, Joseph D. Rowley, Zhanglian Hong, Ayyankkannu Manivannan, Alan D. Bristow, and Nianqiang $\mathrm{Wu}$

12. "Ag@ $\mathrm{Cu}_{2} \mathrm{O}$ Core-Shell Nanoparticles as Visible-Light Plasmonic Photocatalysts" ACS Catalysis, 2013, 3, 1, 47-51

Jiangtian Li, Scott K. Cushing, Joeseph Bright, Fanke Meng, Tess R. Senty, Peng Zheng, Alan D. Bristow, and Nianqiang Wu 